Florida International University FIU Digital Commons

$10-31-2013$

\title{
Regulation of Rab5 GTPase activity during Pseudomonas aeruginosa-macrophage interaction
}

Sushmita Mustafi

Florida International University, smust001@fiu.edu

DOI: $10.25148 /$ etd.FI13121213

Follow this and additional works at: https:// digitalcommons.fiu.edu/etd

Part of the Immunology and Infectious Disease Commons, and the Pathogenic Microbiology Commons

\section{Recommended Citation}

Mustafi, Sushmita, "Regulation of Rab5 GTPase activity during Pseudomonas aeruginosa-macrophage interaction" (2013). FIU Electronic Theses and Dissertations. 1016.

https://digitalcommons.fiu.edu/etd/1016 


\section{FLORIDA INTERNATIONAL UNIVERSITY}

Miami, Florida

\section{REGULATION OF RAB5 GTPASE ACTIVITY DURING PSEUDOMONAS AERUGINOSA-MACROPHAGE INTERACTION}

A dissertation submitted in partial fulfillment of

the requirements for the degree of

DOCTOR OF PHILOSOPHY

in

BIOLOGY

by

Sushmita Mustafi

2013 
To: Dean Kenneth G. Furton

College of Arts and Sciences

This dissertation, written Sushmita Mustafi, and entitled Regulation of Rab5 GTPase activity during Pseudomonas aeruginosa-macrophage interaction, having been approved in respect to style and intellectual content, is referred to you for judgment.

We have read this dissertation and recommend that it be approved.

Lidia Kos

David Kuhn

John Makemson

Rita Mukhopadhyay

Fernando Noriega

M. Alejandro Barbieri, Major Professor

Date of Defense: October 30, 2013

The dissertation of Sushmita Mustafi is approved.

Dean Kenneth D. Furton

College of Arts and Sciences

Dean Lakshmi N. Reddi

University Graduate School

Florida International University, 2013 
(C) Copyright 2013 by Sushmita Mustafi

All rights reserved. 


\section{DEDICATION}

To my loving family 


\section{ACKNOWLEDGMENT}

I wish to express my gratitude and heartfelt appreciation to my advisor, Dr. M. Alejandro Barbieri, for being a great mentor, teacher and motivator. He has always encouraged and inspired me through the wonders and frustrations of scientific research and supported me through out the endeavor. I thank him for all his scientific guidance, for his tolerance and understanding.

Sincere thanks to all my Ph.D. committee members Dr. Lidia Kos, Dr. David Kuhn, Dr. John Makemson, Dr. Rita Mukhopadhyay, and Dr. Fernando Noriega for their kind support, valuable suggestions and critical evaluations.

I would take this opportunity to thank Dr. Phil Stahl, Dr. Dara Frank and Dr. Joan Olson for contributing expertise and valuable resources.

I would also like to thank Maria-Luisa Veisaga (MLV), and all the past and present members of Dr. Barbieri's lab for helping and sharing; for making this journey fun and memorable.

Above all, I want to express my gratitude to my parents, my brother, my husband and all the loved ones for their endless support. 


\title{
ABSTRACT OF THE DISSERTATION \\ REGULATION OF RAB5 GTPASE ACTIVITY DURING PSEUDOMONAS \\ AERUGINOSA-MACROPHAGE INTERACTION
}

\author{
by
}

\author{
Sushmita Mustafi
}

Florida International University, 2013

Miami, Florida

\section{Professor M. Alejandro Barbieri, Major Professor}

Pseudomonas aeruginosa is a Gram-negative opportunistic pathogen. Several antibiotic resistant strains of $P$. aeruginosa are commonly found as secondary infection in immunecompromised patients leaving significant mortality and healthcare cost. Pseudomonas aeruginosa successfully avoids the process of phagocytosis, the first line of host defense, by secreting several toxic effectors. Effectors produced from $P$. aeruginosa Type III secretion system are critical molecules required to disrupt mammalian cell signaling and holds particular interest to the scientists studying host-pathogen interaction. Exoenzyme $\mathrm{S}($ ExoS) is a bi-functional Type III effector that ADP-ribosylates several intracellular Ras (Rat sarcoma) and Rab (Response to abscisic acid) small GTPases in targeted host cells. The Rab5 protein acts as a rate limiting protein during phagocytosis by switching from a GDP- bound inactive form to a GTP-bound active form. Activation and inactivation of Rab5 protein is regulated by several Rab5-GAPs (GTPase Activating Proteins) and Rab5-GEFs (Rab5-Guanine nucleotide Exchange Factors). Some pathogenic bacteria have shown affinity for Rab proteins during infection and make their way inside the cell. This dissertation demonstrated that Rab5 plays a critical role during 
early steps of $P$. aeruginosa invasion in J774-Eclone macrophages. It was found that live, but not heat inactivated, $P$. aeruginosa inhibited phagocytosis that occurred in conjunction with down-regulation of Rab5 activity. Inactivation of Rab5 was dependent on ExoS ADP-ribosyltransferase activity, and more than one arginine sites in Rab5 are possible targets for ADP-ribosylation modification. However, the expression of Rin1, but not other Rab5GEFs (Rabex-5 and Rap6) reversed this down-regulation of Rab5 in vivo. Further studies revealed that the C-terminus of Rin1 carrying Rin1:Vps9 and Rin1:RA domains are required for optimal Rab5 activation in conjunction with active Ras. These observations demonstrate a novel mechanism of Rab5 targeting to phagosome via Rin1 during the phagocytosis of $P$. aeruginosa. The second part of this dissertation investigated antimicrobial activities of Dehydroleucodine (DhL), a secondary metabolite from Artemisia douglasiana, against $P$. aeruginosa growth and virulence. Populations of several $P$. aeruginosa strains were completely susceptible to $\mathrm{DhL}$ at a concentration between $0.48 \sim 0.96 \mathrm{mg} / \mathrm{ml}$ and treatment at a threshold concentration $(0.12 \mathrm{mg} / \mathrm{ml})$ inhibited growth and many virulent activities without damaging the integrity of the cell suggesting anti-Pseudomonas activity of $\mathrm{DhL}$. 


\section{TABLE OF CONTENTS}

CHAPTER

PAGE

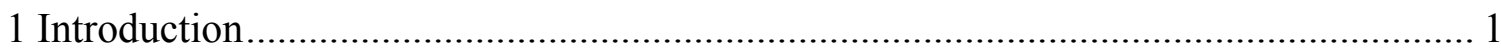

1.1 Phagocytosis - the "Trojan horse" mechanism of immune defense system............. 2

1.2 Mechanism of Phagocytosis ............................................................................... 3

1.2.1 Opsonized and non-opsonized phagocytic particles: To see or not to see......... 3

1.2.2 Phagocytic receptors: Standing tall............................................................. 4

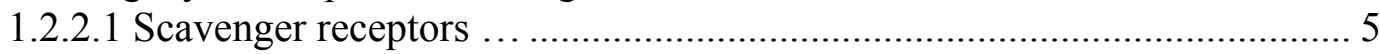

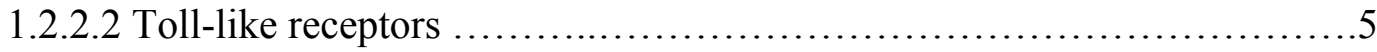

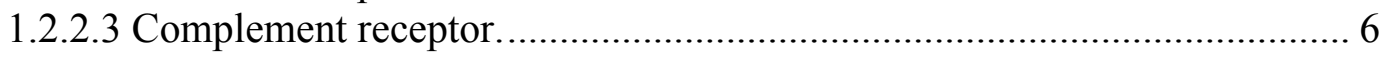

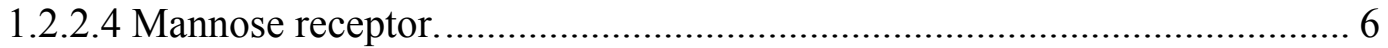

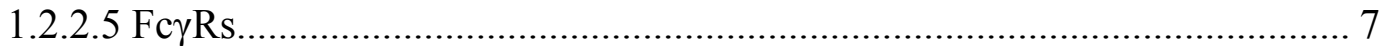

1.2.3 Phagosome formation and maturation: the full circle...................................... 9

1.2.3.1 Regulators of phagosome creation: supporting crew ................................ 11

1.2.4 Rabs and others in phagosome sorting: controllers of busy traffic................... 15

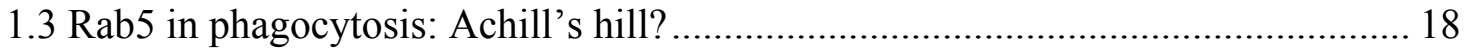

1.3.1 Rab5 modulation by pathogens: escaping early death .................................. 211

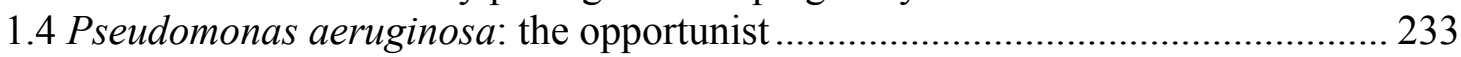

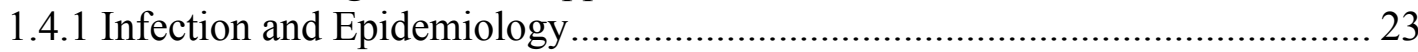

1.4.2 Role of seudomonas aeruginosa virulence factors in immune evasion............ 24

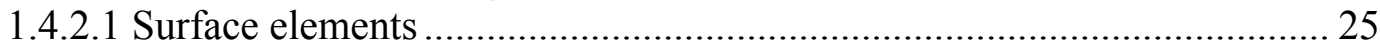

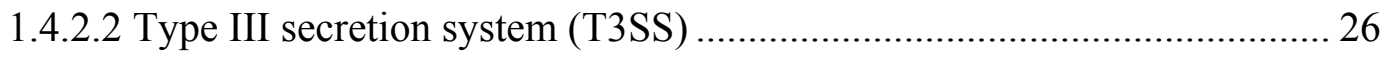

1.4.2.3 Quorum sensing ............................................................................. 29

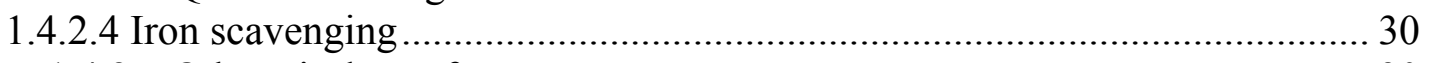

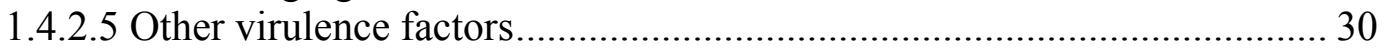

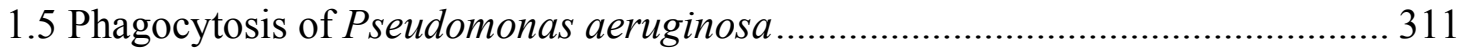

1.6 Antibiotic resistance in Pseudomonas aeruginosa ............................................33

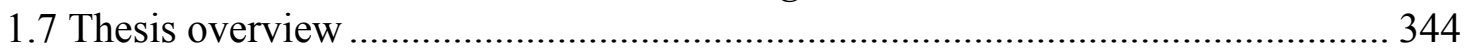

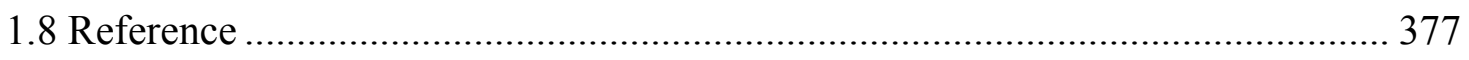

2. Regulations of Rab5 function in macrophages during phagocytosis of live

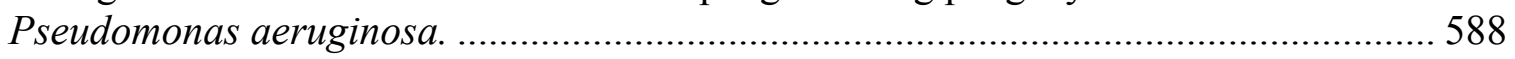

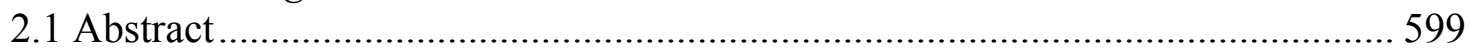

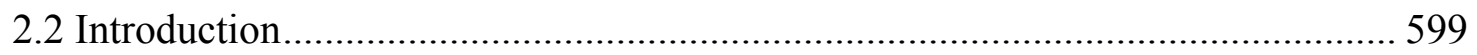

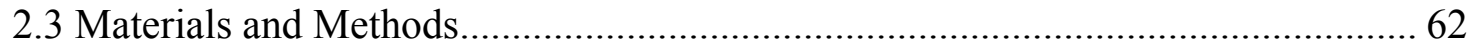

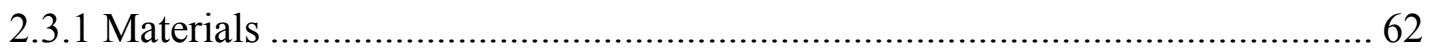

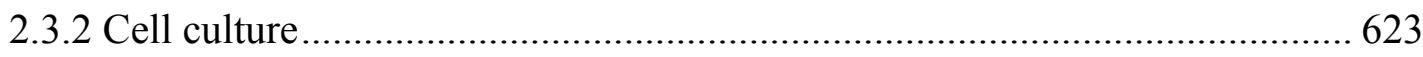

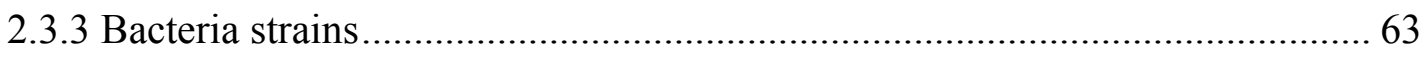

2.3.4 Construction of recombinant pMX-puro retroviruses and cell lines ............... 63

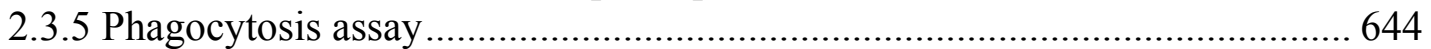

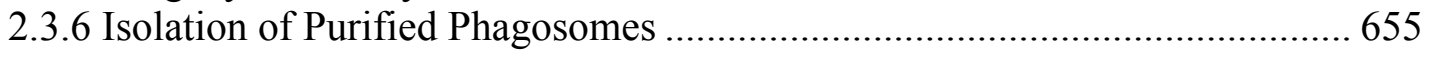

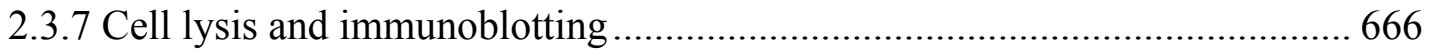

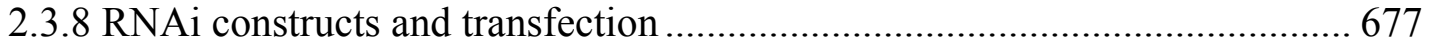




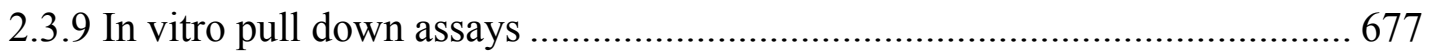

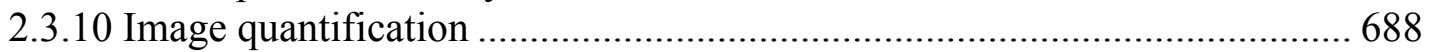

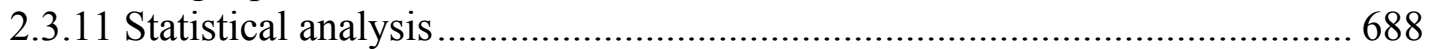

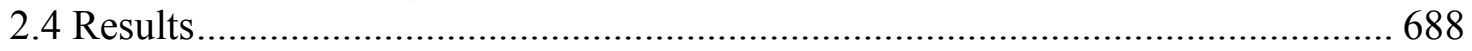

2.4.1 Rab5 is required for phagocytosis of heat-inactivated Pseudomonas

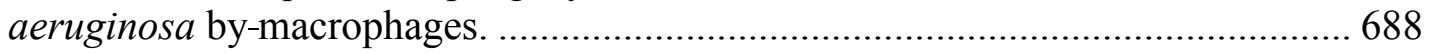

2.4.2 Live Pseudomonas aeruginosa blocks Rab5 activation in macrophages. ..... 711

2.4.3 Exotoxin S plays a critical role in Rab5 activation during phagocytosis of

Pseudomonas aeruginosa.

2.4.4 Selective role of Rab5-GEFs during internalization of Pseudomonas

aeruginosa.

2.4.5 Rin1 expression partially reverses the negative effect of Pseudomonas aeruginosa on Rab5 activation in macrophages.................................................. 788

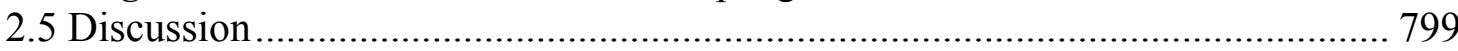

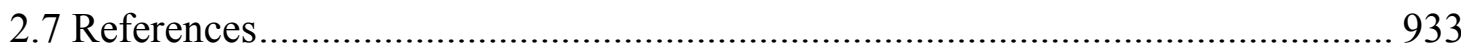

3. Identification and characterization of ADP-ribosylation sites on Rab5 by Pseudomonas aeruginosa type III secretion protein ExoS. .......................................................... 988

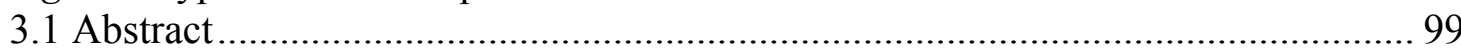

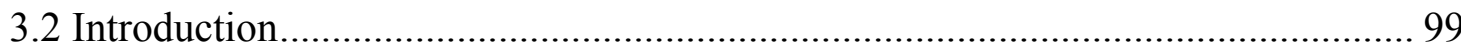

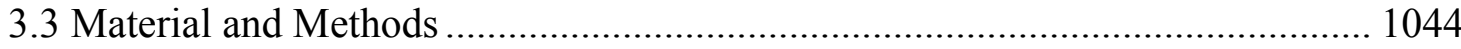

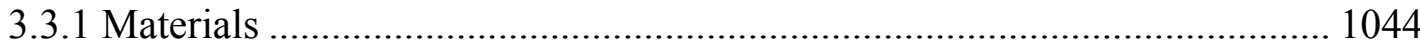

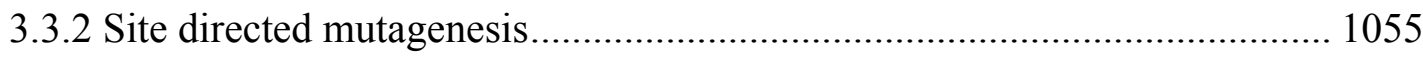

3.3.3 Expression and purification of recombinant proteins ................................. 1055

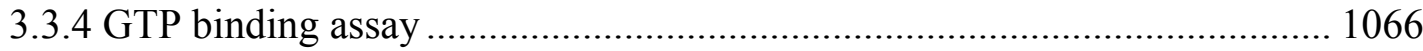

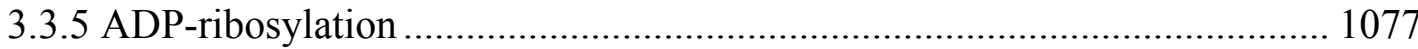

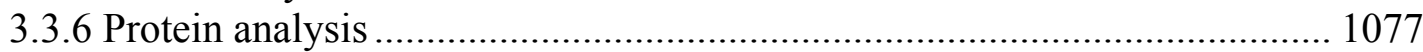

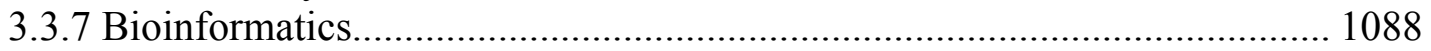

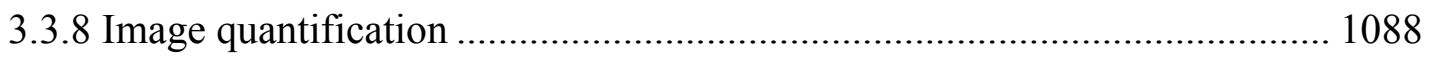

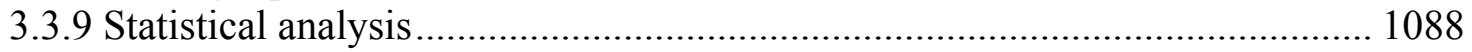

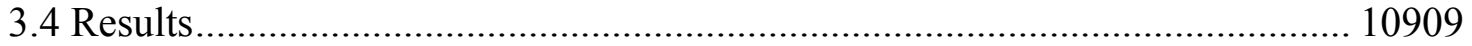

3.4.1 Three arginine sites in Rab5 are conserved implying their importance in Rab5

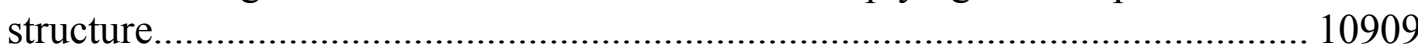

3.4.2 Preparation and purification of several single arginine mutated Rab5..... 10909

3.4.3 Modification of recombinant Rab5 protein didn't disrupt electrophoretic

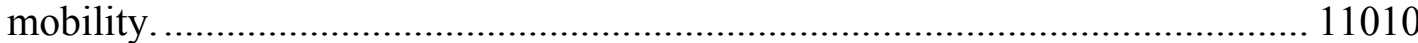

3.4.4 GTP-binding ability among mutated Rab5 protein..................................... 1100

3.4.5 ADP-ribosylation status of arginine mutated Rab5 …................................ 1111

3.4.6 Discrepancies in Rab5-GTP binding post ADP-ribosylation modification. 1111

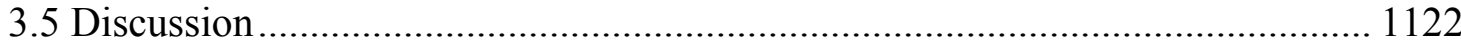

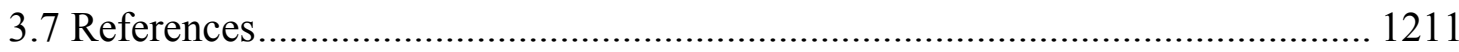

4. The dynamics of Rin1 and Ras in the activation of Rab5 during phagocytosis of Pseudomonas aeruginosa .............................................Error! Bookmark not defined.7

4.1 Abstract.............................................................................................. 1288 


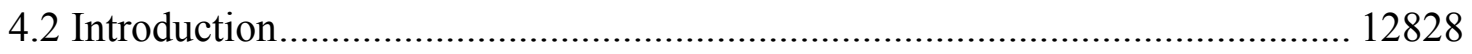

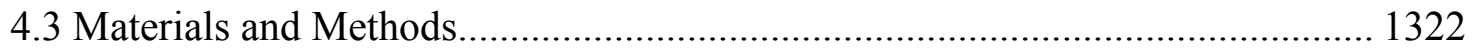

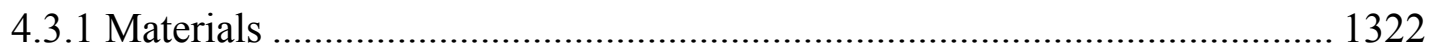

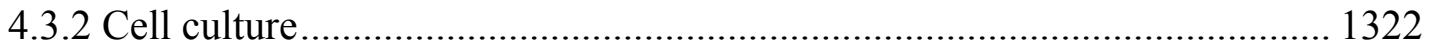

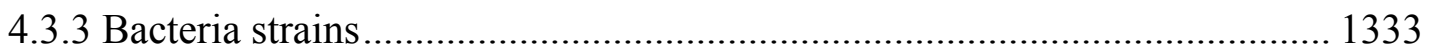

4.3.4 Construction of recombinant pMX-puro retroviruses and cell lines ........... 1333

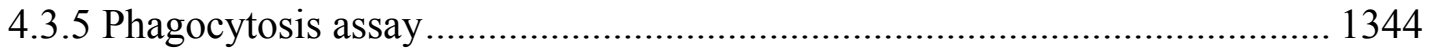

4.3.6 Bacterial survival assay....................................................................... 1355

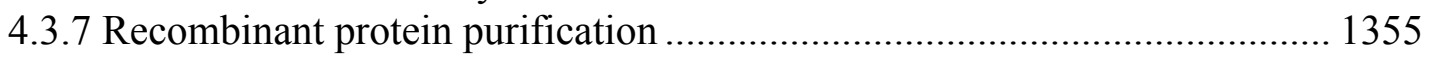

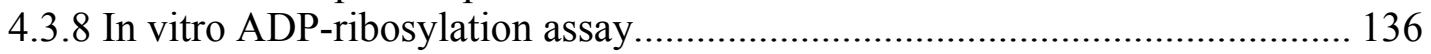

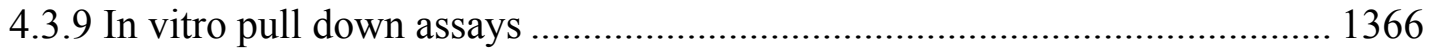

4.3.10 Ras-Rin1 Co-Immuno Precipitations in Vivo............................................. 137

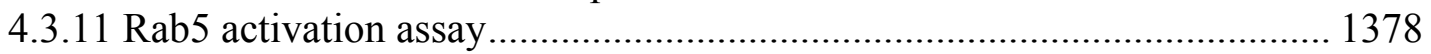

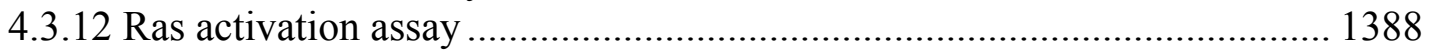

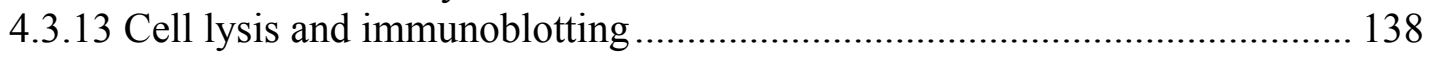

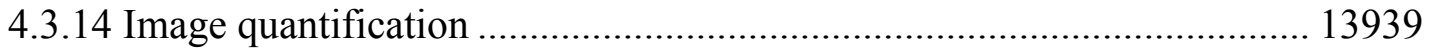

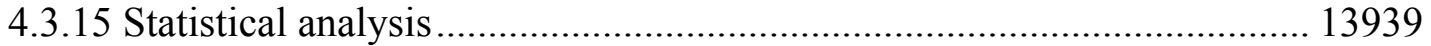

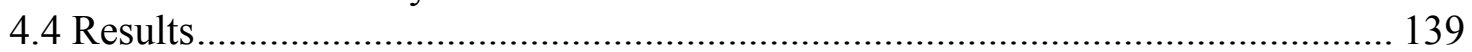

4.4.1 Rab5 activation is interrupted shortly after initiation of live Pseudomonas

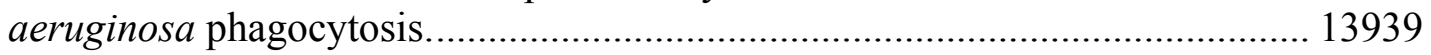

4.4.2 Rin1: FL over-expression in macrophage cells help to retain Rab5-active form at the early phagocytic time points during Pseudomonas aeruginosa invasion. .... 141 4.4.3 Selective role of Rin1 domains in Rab5 activation during internalization of live

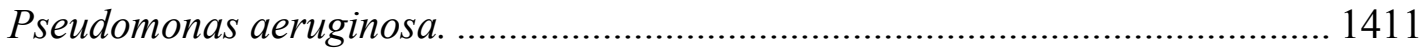

4.4.4 Ras is active in early phase of Pseudomonas aeruginosa phagocytosis...... 1422

4.4.5 Rin1 FL interacts with Ras in Pseudomonas aeruginosa phagocytosis. ..... 1433 4.4.6 Carboxyl terminal domain of Rin1 protein selectively interacts with modified and unmodified Ras and Rab5 protein in vitro.................................................... 144

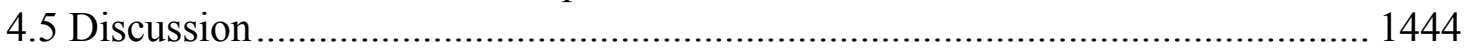

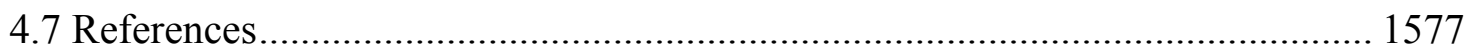

5. Antimicrobial activity of Dehydroleucodine against Pseudomonas aeruginosa...... 1611

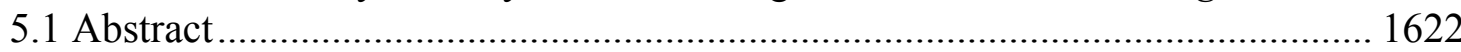

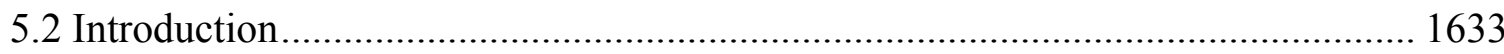

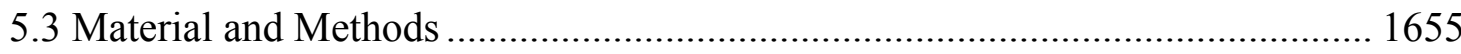

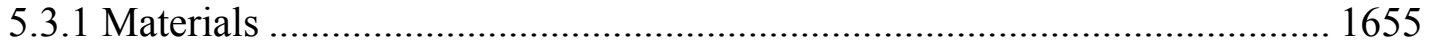

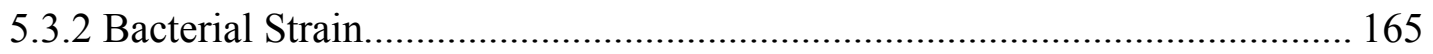

5.3.3 Determination of Minimum Inhibitory Concentration .................................... 1666

5.3.4 DhL treatment (threshold concentration) .................................................... 166

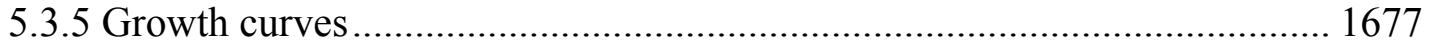

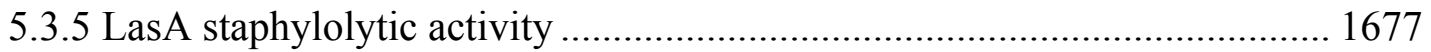

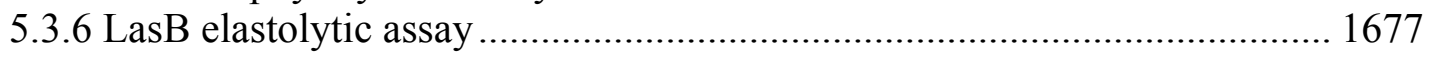

5.3.7 Polyvinyl chloride biofilm formation attachment assay .............................. 1688

5.3.8 Pyocyanin assay ................................................................................ 1688

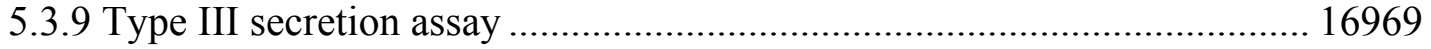




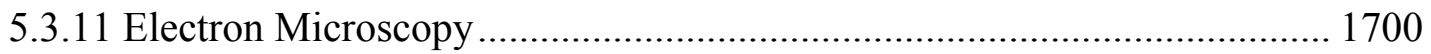

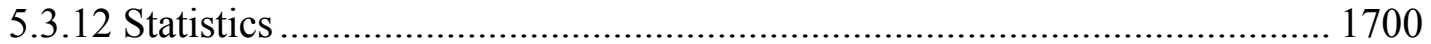

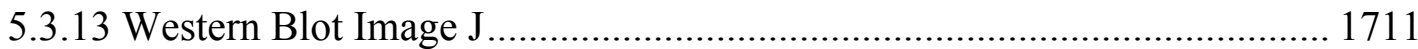

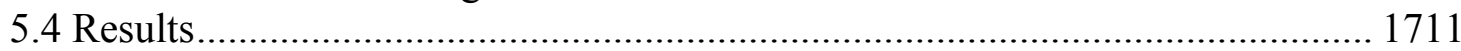

5.4.1 Activity of DhL against Pseudomonas aeruginosa .................................... 1711

5.4.2 Electron Microscopy analysis of Pseudomonas aeruginosa cellular structure

5.4.3 Influence of threshold concentration of $\mathrm{DhL}$ on growth rate

5.4.4 Activity of DhL against $P$ seudomonas aeruginosa virulence factors: Elastase

A (LasA) and Elastase B (LasB)................................................................... 1722

5.4.5 DhL has an inhibitory effect on biofilm formation...................................... 1733

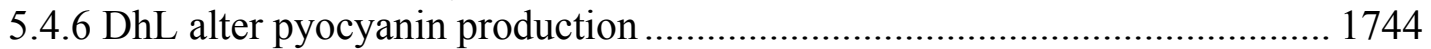

5.4.7 DhL inhibits Type III secretion effectors................................................. 1744

5.4.8 DhL treated Pseudomonas aeruginosa and apoptosis ............................... 1744

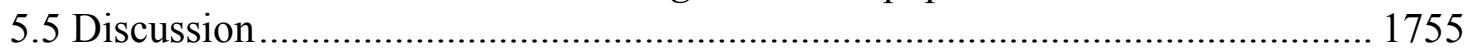

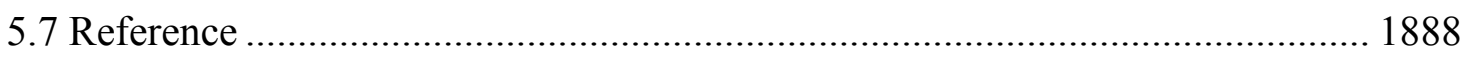

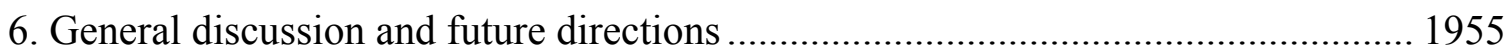

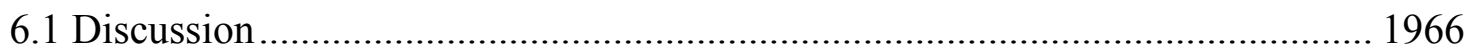

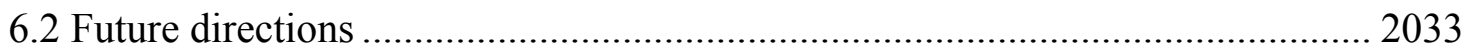

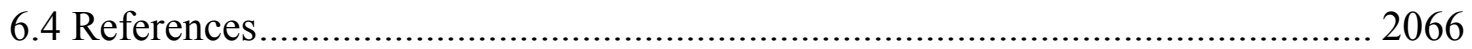

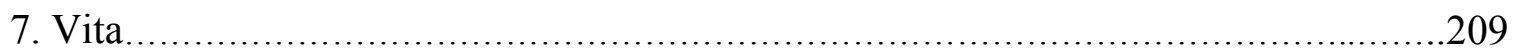




\section{LIST OF FIGURES}

FIGURE

PAGE

Figure 1: Receptors involved in opsonized or non-opsonized phagocytosis................... 4

Figure 2: Phagocytic events in macrophage cell....................................................... 17

Figure 3: Pathogenic bacteria mediated subversion of phagocytosis. ........................... 22

Figure 4: Virulence factors of Pseudomonas aeruginosa ............................................ 25

Figure 5: Schematic representations of Pseudomonas aeruginosa Type III secretion

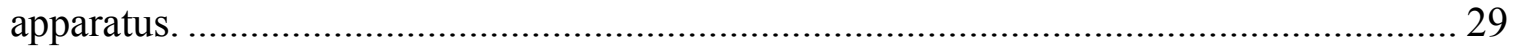

Figure 6: Effect of Rab proteins on invasion of heat-inactivated $P$. aeruginosa in

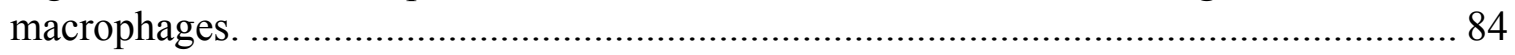

Figure 7: Effect of Rab5 on live P. aeruginosa invasion in macrophages. .................... 86

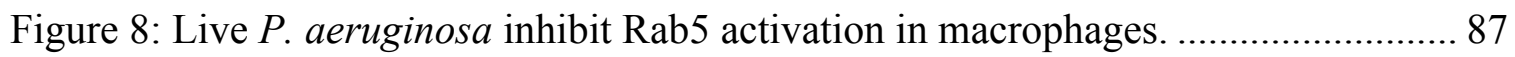

Figure 9: Selective effect $P$. aeruginosa exotoxins on Rab5 activation in macrophages. 88

Figure 10: Effect of ExoS GAP or ADPr activity on P. aeruginosa invasion.................. 89

Figure 11: Effect of Rab5-GEFs in the internalization of $P$. aeruginosa ...................... 91

Figure 12: Effect of Rab5-GFEs on Rab5 activity during phagocytosis of P. aeruginosa.

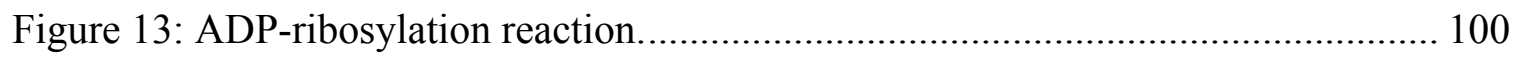

Figure 14: Schematic representations of different domains of P. aeruginosa ExoS..... 104

Figure 15: Rab5 multiple sequence alignment.................................................. 115

Figure 16: Arginine mutation sites on Rab5 .................................................... 116

Figure 17: Purification of mutated Rab5 proteins................................................. 117

Figure 18: GTP binding analysis of Rab5 mutants............................................ 118

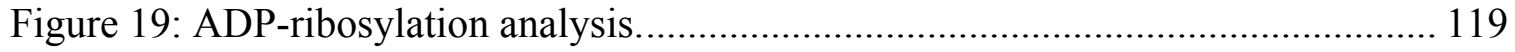


Figure 20: GTP binding status of ADP-ribosylated Rab5.

Figure 21 Schematic representation of different Rin1 protein constructs.

Figure 22: Shortened life span of Rab5-GTP bound form in the event of early endocytosis during live $P$. aeruginosa invasion.

Figure 23: Effect of Rin1 FL, a Rab5-GEF on Rab5 activity during phagocytosis of $P$. aeruginosa.

Figure 24: Determination of live (L) $P$. aeruginosa invasion and survival inside macrophage in presence of Rin1 FL and its domains.

Figure 25: Activation of Ras during phagocytosis of $P$. aeruginosa.

Figure 26: Rin1 binds to Ras during phagocytosis of $P$. aeruginosa. 154

Figure 27: ADP-ribosylated Ras and Rab5 interaction with Rin1 R3 in vitro. 155

Figure 28: Chemical structure of Dehydroleucodine isolated from Artemisa douglasiana.

Figure 29: Electron microscopy analysis of DhL treated P. aeruginosa. 180

Figure 30: Influence of DhL on growth of P. aeruginosa. 181

Figure 31: Effect of DhL on P. aeruginosa strain PAO1 protease activities. 182

Figure 32: Effect of DhL on P. aeruginosa biofilm formation 184

Figure 33: Pyocyanin production in $\mathrm{DhL}$ treated $P$. aeruginosa 185

Figure 34: Effect of DhL on TypeIII secretion. 186

Figure 35: Effect of DhL on P. aeruginosa induced apoptosis. 187

Figure 36: Model for Ras and Rin1 facilitated Rab5 activation during P. aeruginosa phagocytosis. 


\section{LIST OF ABBREVIATIONS AND ACRONYMS}

AA

Arachidonic Acid

ADP Adenosine Di Phosphate

ADPr Adenosine diphosphate (ADP)-ribosylation

ADPRT

ADP-Ribosyl-Transferase

$\mathrm{ARF}$

ADP Ribosylation Factor

ARNO

ARF Nucleotide binding site Opener

BSA Bovine Serum Albumin

$\mathrm{Ca}^{+2}$

Calcium

cAMP cyclic Adenosine Mono Phosphate

$\mathrm{Cbl}$ .Cannabinoid Receptor 1

CDC42 Cell Division Control protein 42 homolog $\mathrm{CF}$. Cystic Fibrosis CR Complement Receptors

Crk CT10-regulator of kinase

CrkII CT10 regulator of kinase II

DAG Diacylglycerol

EEA1 Early Endosome Antigen 1 ERK Extracellular signal Regulated Kinase

Exo Exoenzyme

Fc Fragment, crystallizable Region

FcR Fc Receptor

FL Full Length 
FYVE......Zinc finger domain named after cysteine rich proteins Fab1, YOTB, Vac1 and GAP GTPase Activating Protein

Gab2 Grb2 associated binding protein2 GDI. Rab GDP dissociation inhibitor GDI. GDP dissociation Inhibitors GDP Guanosine di phosphate GEF Guanine nucleotide Exchange Factor GFP Green Fluorescent Protein Grb2 Growth factor Receptor Bound Protein 2 GTP Guanosine Tri Phosphate

Ig Immunoglobulin

IL Interleukin

IP3. Inositol Tri Phosphate

ITAM Immunoreceptor signaling motif

JNK c-Jun Kinase

LAMP Lysosome-Associated Membrane Proteins LOX-1 .Low-density lipoprotein receptor-1 LPS Lipopolysaccharide MAPK Mitogen Activated Protein Kinase MyD88 Myeloid Differentiation factor 88

NAD .Nicotinamide Adenine Dinucleotide $\mathrm{NADPH}$ .nicotinamide adenine dinucleotide phosphate $\mathrm{NF}-\kappa \mathrm{B}$ Nuclear Factor Kappa-light-chain-enhancer of activated B cells 
NSF

Soluble N-ethylmaleimide-sensitive Factor

p.i. post infection

PAMP Pathogen-Associated Molecular Pattern

PARP .Poly-ADP-Ribose-Polymerase PBS Phosphate Buffered Saline

PH. .Pleckstrin Homology

$\mathrm{PI}(3,4) \mathrm{P} 2$ Phosphatidyl Inositol $(3,4)$ biphosphate

$\mathrm{PI}(3,4,5) \mathrm{P} 3$ Phosphatidyl Inositol $(3,4,5)$ triphosphate

$\mathrm{PI}(4,5) \mathrm{P} 2$ Phosphatidyl inositol-4,5-bisphosphate PI3K .Phosphoinositide 3 Kinase

PI3P Phosphatidyl inositol 3 phosphate PIP3 Phosphatidyl inositol-3,4,5-triphosphate

PKC Protein Kinase C

PLA. phospholipase A

PLC .Phospholipase C

PLD. Phospholipase D PRR Pattern Recognition Receptors PTEN Phosphatase and Tensin homolog protein QS Quorum Sensing

RA. Ras Association domain

Rab. Response to abscisic acid

RABEX-5. Rabaptin-5 associated Exchange factor for Rab5

RAP6. Rab5 Activating Protein 6 
Ras

Rat sarcoma

REP.

Rab Escort Proteins

Rho-GAP Rho-GTPase-activating protein

RILP. Rab7 Interacting Lysosomal Protein

RIN1.... Ras interference/interaction 1

SDS-PAGE Sodium dodecyl sulfate polyacrylamide gel electrophoresis

$\mathrm{SH} 2$ Src Homology 2

SHIP-1 SH2 containing Inositol Phosphatase-1

SNARE ..Soluble N-ethylmaleimide-sensitive factor (NSF) Attachment protein Receptor T3SS. Type III Secretion System

TAK1 Transforming growth factor beta (TGF $\beta$ ) Activating Kinase 1 TGF $\beta$ Transforming Growth Factor beta

Tiam1 T-cell lymphoma invasion and metastasis 1

TLR Toll-like receptors

TNF- $\alpha$ Tumor Necrosis Factor alpha

VAMP3 Vesicle Associated Membrane Protein 3

VAP Ventilator-Associated Pneumonia

VPS34. ..Vacuolar Sorting Protein 34

VPS9 Vacuolar Sorting Protein 9

WASP Wiskott-Aldrich syndrome protein WAVE WASP family Verproline-homologous protein

WT. Wild Type $\mathrm{ZnF}$ .Zinc Finger 


\section{Chapter 1}

\section{Introduction}

This chapter aims to introduce the concept of cell signaling cascade during phagocytosis focusing on the signaling mechanisms of Rab5 GTPase, a brief discussion on modulation of Rab5 protein by Gram negative bacteria, and a short review of Pseudomonas aeruginosa. Overall the information creates an outline for the research hypothesis. Partial content of this chapter has been published (Mustafi, Barbieri et al. 2013). 


\subsection{Phagocytosis - the "Trojan horse" mechanism of immune defense system}

Eukaryotic cells have evolved elegant mechanism of endocytosis, which internalizes fluids (pinocytosis) or particulate constituents (phagocytosis) from their environment. Phagocytosis is a process delineated as uptake of large particles, typically $0.5-5.0 \mu \mathrm{m}$ in diameter (Kinchen and Ravichandran 2008). The process serves as a mode of nutrition in unicellular organisms, but complex organisms have taken advantage of the phagocytic machinery in their defense system. As a part of the immune system mobile phagocytic cells survey tissues for foreign particles and engage in pitched battles with potential pathogens (Tauber 2003). In mammals, phagocytosis is primarily performed by cells such as monocytes, macrophages, neutrophils and dendritic cells, which play important roles in both innate and adaptive immunity. Interestingly, in nematodes like Caenorhabditis elegans phagocytosis is studied extensively for its role in initiating programmed cell death (Reddien, Cameron et al. 2001).With the advantage of having a limited repertoire of genes, the slime mold Dictyostelium discoideum has served as a model organism for studies involving mechanisms of phagocytosis for several years (Bozzaro and Eichinger 2011). Phagocytosis is initiated by the recognition and binding of a foreign particle to receptors on the cell surface (Shaw and Griffin 1981; Angus, Evans et al. 2010). In order to discriminate between non-self and self-agents, professional phagocytes express a restricted number of phagocytic receptors that either recognize conserved motifs on pathogens or on foreign proteins coated with host immunoglobins (opsonins) (Mehta 1976). Upon contact with the phagocytic substance or microbe, many parallel signaling pathways are simultaneously activated as a response from phagocytes prior to internalization (Kwiatkowska and Sobota 1999). The array of signals triggers cellular 
processes such as cytoskeletal rearrangement, altered membrane trafficking, activation of killing mechanisms, production of cytokines, chemokines and apoptosis (Aderem and Underhill 1999). Among a variety of phagocytic receptors, most of the understanding of the signaling pathways leading to phagocytosis comes from the studies of the Fc Receptors (FcR).

\subsection{Mechanism of Phagocytosis}

\subsubsection{Opsonized and non-opsonized phagocytic particles: To see or not to see}

Several types of receptors participate in recognizing opsonin-coated or uncoated particles (Figure1). Opsonin-coated particles (opsonized) are either coated with hostderived proteins like mannose binding proteins, surfactant protein $\mathrm{A}$, immunoglobulins and complement fragments, which are recognized by complement receptors (CR) such as CR1, CR3 and CR4 or FcrRs that increase binding and recognition of an antigen by the immune cells (Aderem and Underhill 1999). Several cellular pattern rocognition receptors (PRRs) like mannose and scavenger receptors (Class A and Class B) and Ctype lectin-like receptor recognize non-opsonized surface components of pathogens also called pathogen-associated molecular patterns (PAMPs) such as bacterial lipopolysaccharides, mannans and integrins (Janeway 1989; Sastry and Ezekowitz 1993) or phosphatidyl serine by apoptotic cells to trigger phagocytosis (Ofek, Goldhar et al. 1995; Kwiatkowska and Sobota 1999). 


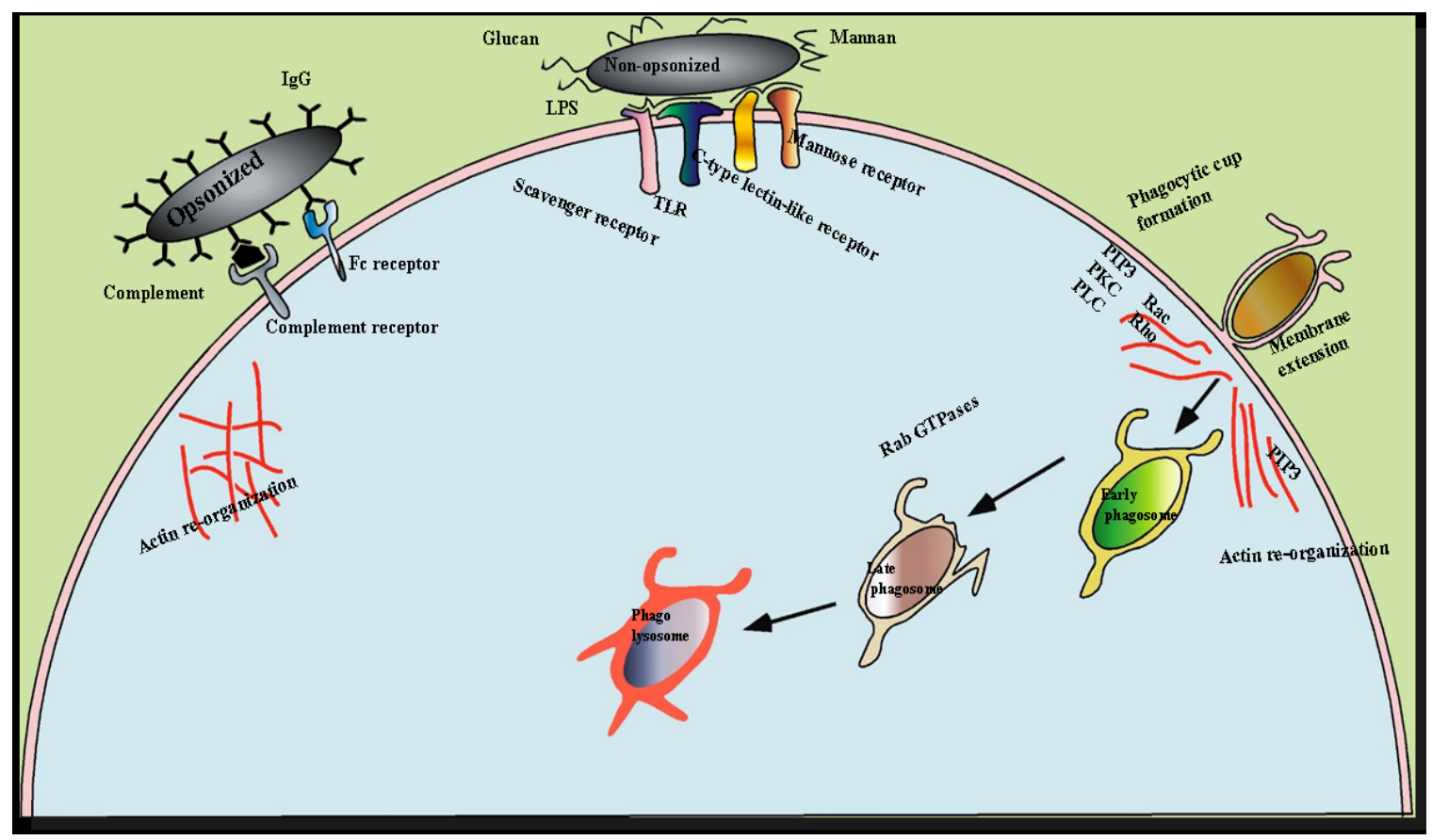

Figure 1: Receptors involved in opsonized or non-opsonized phagocytosis.

Diagramatic representation of phagocytic cell showing receptors involved in the process of opsonized and non-opsonized particle ingestion, recognition molecules attached to opsonized and non-opsonized particles and the events of phagocytosis from phagosome formation to phago-lysosome fusion.

\subsubsection{Phagocytic receptors: Standing tall}

Receptors on the cell surfaces of professional phagocytes trigger and initiate a series of signaling events during the process of phagocytosis (Targowski and Klucinski 1985). Myeloid cells such as macrophages and dendritic cells function as professional phagocytes and express a range of PRRs for pathogen recognition, induction of adaptive immunity, or clearing apoptotic/necrotic and modified cellular metabolites (Gordon, Molyneux et al. 2001; Larsson, Fonteneau et al. 2001). 
1.2.2.1 Scavenger receptors are involved in opsonin-independent receptor-mediated phagocytosis with a wide range of ligands ranging from lipoteichoic acid of Grampositive bacteria, lipopolysaccharides in Gram-negative bacteria, modified low density lipoproteins, double stranded RNA, double stranded DNA and apoptotic cells (Kodama, Doi et al. 1996). Scavenger receptors are categorized into several classes (class A types I and II, class B, class C, class E, also known as lectin-like oxidized low-density lipoprotein receptor-1 (LOX-1), and class F) determined by the presence or absence of signature structural domains such as collagenous domain, cysteine rich domain, C-typelectin domain or alpha helical coiled-coil domain (Peiser and Gordon 2001). Class A scavenger receptors are triggered by modified lipoprotein or microbial cell surface molecules. They induce gene transcription and cytokine release with signaling response via tyrosine phosphorylation and activation of the Mitogen Activated Protein Kinase (MAPK) pathway (Coller and Paulnock 2001). In contrast, macrophage receptors with collagenous structure (Cavalli, Corti et al. 2001), a class A scavenger receptor, expressed by only a sub population of macrophages recognize only bacterial polysaccharides (Pikkarainen, Brannstrom et al. 1999; Elshourbagy, Li et al. 2000), whereas class B1 scavenger receptor is a cellular receptor for high-density lipoprotein (Silverstein, Li et al. 2010). Invasion of Staphylococcus aureus in osteoblasts and Listeria monocytogenes in the spleen and liver involves various scavenger receptors (Thomas, Li et al. 2000) (Suzuki, Kurihara et al. 1997).

1.2.2.2 Toll-like receptors (TLR) can only recognize conserved microbial domains (Akira 2001; Janeway and Medzhitov 2002; Akira 2003) or bacterial DNA (Takeda, Kaisho et al. 2003). To date, more than 10 members of the TLR family have been 
reported in mammals recognizing a variety of PAMPs (Akira, Uematsu et al. 2006; Medzhitov 2007). Upon stimulation, they initiate an effective cascade leading to the release of antimicrobial peptides and cytokines ( $\mathrm{O}^{\prime} \mathrm{Neill}$ 2002), including adapter molecule MyD88, Transforming growth factor beta (TGF $\beta$ ) Activating Kinase 1 (TAK1), and TAK1-binding proteins 1 and 2 (Akira 2003). The receptor-adapter interaction, in turn, propagates signal for the activation of Nuclear Factor Kappa-light-chain-enhancer of activated B cells (NF-KB), MAPK, phosphatidylinositol 3-kinase (PI3K) and c-Jun Kinase (JNK) leading to proper display of cellular defense (Ojaniemi, Glumoff et al. 2003).

1.2.2.3 Complement receptor mediated phagocytosis is a passive process that occurs by a variation of the classic zipper model (Aderem and Underhill 1999). A series of complement receptors (such as CR1) are expressed on the surface of macrophages that recognize complement proteins (such as $\mathrm{C} 3 \mathrm{~b}$ or $\mathrm{C} 3 \mathrm{bi}$ ), which are present in the serum (Carroll 1998). Complement Receptor 1 (CR1) binds C3b, C4b and C3bi opsonized particles while CR3 and CR4 bind specifically to C3bi resulting in the phagocytosis of complement-coated proteins/cells (Trinidad, de la Puerta et al. 2006; Patel and Harrison 2008). Unlike FcR-mediated phagocytosis, complement receptors do not elicit the release of inflammatory mediators (Allen and Aderem 1996).

1.2.2.4 Mannose receptor is a transmembrane lectin scavenger receptor implicated in the clearance of mannose- and fucose-terminated glycoproteins (i.e., lysosomal hydrolases). It also recognizes numerous pathogens such as Candida albicans, Pneumocystis jirovecii, and the dengue virus. The receptor initiates phagocytosis by recruiting $\mathrm{F}$ actins, talin, Protein Kinase $\mathrm{C} \alpha(\mathrm{PKC} \alpha)$, microtubule associated protein (MARKS) and Myosin I at 
the nascent phagosome formation site (Ezekowitz, Sastry et al. 1990; Stahl and Ezekowitz 1998). Mannose receptors are also known to play a critical role in the development of crescentic glomerulonephritis by augmenting Fc-mediated function (Chavele, Martinez-Pomares et al. 2010).

1 2.2.5 Fc $\gamma \mathbf{R s}$ are a family of membrane proteins that comprise three subunits: Fc $\gamma \mathrm{RI}$

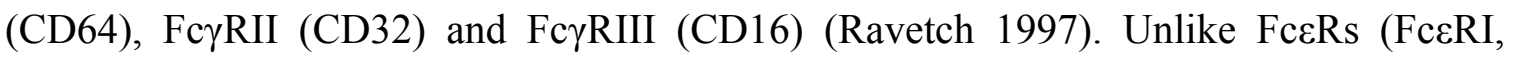
FceRII) and Fc $\alpha R$ (FcaRI) that interact with immunoglobulin (Ig) E (Larsen, DiGennaro et al. 2000) and IgA opsonized particles, respectively (Aderem and Underhill 1999), Fc $\gamma$ Rs interact with the constant region of the IgG and aid in opsonization (Caron and Hall 1998). The Fc $\gamma \mathrm{R}$ receptor mediated phagocytosis has been studied extensively and is a three-step process, i.e., binding of ligand coated particles with Fc $\gamma \mathrm{R}$, clustering of receptors, and triggering a signal cascade leading to actin rearrangement and particle engulfment (Jiang, Zhong et al. 2002). The activation of Fc $\gamma \mathrm{R}$ requires bivalent or multivalent IgG coated ligands (Jones, Nusbacher et al. 1985; Odin, Edberg et al. 1991) leading to lateral clustering of FcyRs. The cytosolic domain of these receptors contains the immunoglobulin gene family tyrosine activation motif (Kooguchi, Hashimoto et al. 1998), characterized by the specific tandem sequence YxxI/L (Anderson, Shen et al. 1990; Takai, Kasama et al. 1994). The Src family of tyrosine kinases (such as Hck, Lyn and Fgr) phosphorylates Immunoreceptor signaling motif (ITAM) and recruits spleen tyrosine kinases (Wilson, Pitt et al. 1987; Reth 1989; Ravetch 1994). Signal amplification is achieved by the Scr homology domain 2 (SH2) of Syk that binds phosphorylated ITAM leading to continued phosphorylation of neighboring ITAMs (Crowley, Costello et al. 1997; Kiefer, Brumell et al. 1998). Investigations also revealed that Syk signaling is 
required for phosphorylation of the regulatory subunit of $\mathrm{p} 85$ and activation of $\mathrm{p} 110 \beta$, the catalytic subunit of Phosphoinositide 3 Kinase (PI3K), which is associated with the accumulation of lipid phosphatidyl inositol-3,4,5-triphosphate (PIP3) from phosphatidyl inositol-4,5-bisphosphate $(\mathrm{PI}(4,5) \mathrm{P} 2)$ in the membrane close to the receptor cluster (Crowley, Costello et al. 1997; Leverrier, Okkenhaug et al. 2003). The cluster also attracts a series of adapter proteins like the transmembrane protein linker activated $\mathrm{T}$ cells (Kiefer, Brumell et al. 1998), Growth factor Receptor Bound Protein 2 (Grb2), CT10 regulator of kinase II (CrkII), Cannabinoid Receptor 1 (Cbl) and Non-Catalytic region of Tyrosine Kinase adaptor protein (Sato, Frank et al. 2003) to the nascent phagocytic cup (Izadi, Erdreich-Epstein et al. 1998; Tridandapani, Lyden et al. 2000; Lee, Cosio et al. 2007). Adaptor proteins play a crucial role in linking downstream signaling molecules of FcyR. Scientist Gu and others identified Grb2 associated binding protein2 (Gab2) as a major element of the FcyR-mediated phagocytic mechanism in macrophages and demonstrated its role in the local generation of PIP3 lipids at the phagocytic cup (Gu, Botelho et al. 2003). Furthermore, it has been shown that, upon activation of Fc $\gamma \mathrm{R}, \mathrm{Gab} 2$ gets phosphorylated and binds to p85 of PI3K, and Shp-2, a SH2 domain-containing tyrosine phosphatase (Gu, Botelho et al. 2003). Other players in the phagocytic signaling process include calcium $\left(\mathrm{Ca}^{+2}\right)$, Rho GTPase, protein kinase C (PKC), phospholipase C (PLC) and phospholipase D (PLD) (Kiener, Rankin et al. 1993; Ghazizadeh and Fleit 1994; Hackam, Rotstein et al. 1997; Aderem and Underhill 1999; Cox, Tseng et al. 1999; Kwiatkowska and Sobota 1999; Lennartz 1999) that control the fusion of vesicles with the plasma membrane, providing membranes for phagosome formation (Celli and Finlay 2002). They are also involved in sealing the phagosome and triggering a series of rapid 
and extensive changes in phagosome maturation, which are characterized by sequential interaction with sorting of early endosomes, late endosomes and lysosomes (Desjardins, Huber et al. 1994; Scott, Furuya et al. 2003). Lee and others showed that during the internalization of large but not small particles, the membrane of the phagocytic cup is almost completely remodeled (Lee, Cosio et al. 2007). It has been demonstrated that in order for phagocytosis to occur by neutrophils, unfolding of the surface membrane wrinkle is necessary (Hallett and Dewitt 2007). Actin polymerization at the phagocytic cup and pseudopod extension can be affected separately, indicating that membrane remodeling is an independent component of the phagocytic response (Lowry, Duchemin et al. 1998; Cox, Tseng et al. 1999). Furthermore, membrane remodeling during phagocytosis is an active and complex process that involves localized pinocytosis (Botelho, Tapper et al. 2002), segregation of membrane components into lipid rafts (Kwiatkowska, Frey et al. 2003), lateral diffusion of signaling molecules (Henry, Hoppe et al. 2004), and the insertion of endomembranes by focal exocytosis (Bajno, Peng et al. 2000; Braun, Wendt et al. 2004).

\subsubsection{Phagosome formation and maturation: the full circle}

The phagosome maturation process is accomplished in a sequential manner with the formation and pinching off of the phagocytic vacuole followed by its interaction with early and late endosomes and finally fusion with the lysosome (Li, Jagannath et al. 2010). Though there is evidence of signaling molecules which accumulates at the site of phagosome formation and controls the process before the pathogenic secretion system brings disruption, the exact role of macrophage cell surface receptors and phagosome maturation is yet not well understood (Kinchen and Ravichandran 2008). The 
contribution of FcR mediated uptake of opsonized prey to phagosome formation has been well documented (Pohlmann, Boeker et al. 1995; Vieira, Botelho et al. 2001) In contrast, the role of TLRs in influencing phagosome maturation is not very clear though TLR 4 and 2 has been speculated to fast track phagosome maturation process (Yates and Russell 2005; Blander and Medzhitov 2006). Also, there are very few studies describing phagosome maturation triggered by complement receptors, but evidence suggests the pathway is $\mathrm{Ca}^{+2}$ dependent (Malik, Denning et al. 2000; Malik, Thompson et al. 2003). Overall, many reviewers suggested that the pivotal role of cell surface receptors is to generate inflammatory or apoptotic signals whereas the phagosome formation and maturation can cross common paths (Vieira, Botelho et al. 2002; Underhill 2005; Kinchen, Doukoumetzidis et al. 2008). The organized signaling stages in FcR mediated phagocytosis follows the zipper model proposed by Griffin and Silverstein (Griffin and Silverstein 1974). The three stages of signaling proposed by the zipper model involve several different molecules and are regulated tightly by catalytic events. Modulation of the levels of phosphorylated proteins and recruitment of molecules necessary for the subsequent steps are localized at the point of receptor clustering. Hence the process is characterized by coordinated stages of lateral diffusion and checkpoint regulated progress (Kinchen and Ravichandran 2008). Formation of a phagosome requires a considerable amount of membrane area, which is provided by the plasma membrane initially. However, additional membrane from intracellular compartments is also supplied by means of the focal exocytosis mechanism (Cox, Lee et al. 2000; Lee, Cosio et al. 2007). Internalization of particles involves reorganization of actin cytoskeleton, recruitment of different classes of myosin, and membrane fusion to complete the phagocytic vacuole 
(Swanson, Johnson et al. 1999; Diakonova, Bokoch et al. 2002; Henry, Hoppe et al. 2004). Initial phosphorylation events generated by FcR activation relays the signal to membrane localized PI(4,5)P2 (Botelho, Hackam et al. 2000), which recruits Rho-family GTPases Rac1 and Cdc42 (Botelho, Teruel et al. 2000), and with the aid of WiskottAldrich syndrome protein (WASP) and WASP family verproline-homologous protein (WAVE) proteins elicit the activity of actin-nucleating complex Arp2/3 (Takenawa and Miki 2001). The spike of actin assembly and phagocytic cup formation is also associated with hydrolysis of PI $(4,5)$ P2 to diacylglycerol (DAG) or PI3K mediated phosphorylation to PIP3. This is followed by activation of Rac, ADP ribosylation factor (ARF) 6 for membrane fusion events (Swanson and Hoppe 2004). Subsequent hydrolysis of PIP3 by Phosphatase and Tensin homolog protein (PTEN) or SH2 containing Inositol Phosphatase-1 (SHIP-1) generates PI(4,5)P2 or PI(3,4)P2 and ushers in the final stage of phagocytic vesicle sealing (Kavran, Klein et al. 1998). Phospholipase A (PLA) 2 mediated release of arachidonic acid (AA) stimulates nicotinamide adenine dinucleotide phosphate-oxidase (NADPH-oxidase) activation leading to completion of phagocytic cup formation. Flash appearance of $\mathrm{PKC} \varepsilon$ is also associated to the membrane folding and fusion event of phagocytic cup (Larsen, Ueyama et al. 2002).

\subsubsection{Regulators of phagosome creation: supporting crew}

The majority of downstream proteins activated by receptors participate in more than one process. Here the discussion covers the role of key proteins in the cascade of phagocytosis as revealed in the biochemistry of actin polymerization/depolymerization, myosin regulation, vesicle fusion and assembly of NADPH oxidase. Type Ia PI3K (there are three main types of PI3Ks) consists of two subunits, a catalytic subunit (p110) and a 
regulatory subunit (p85) (De Camilli, Emr et al. 1996). The PI3K activation triggers the phosphorylation of several PIs, thereby generating Phosphatidyl Inositol $(3,4)$ biphosphate $(\mathrm{PI}(3,4) \mathrm{P} 2)$ or Phosphatidyl Inositol $(3,4,5)$ triphosphate $(\mathrm{PI}(3,4,5) \mathrm{P} 3)$ (Araki, Johnson et al. 1996; Cox, Tseng et al. 1999). Activation of PI3K is crucial for the extension of pseudopods in formation and closure of the phagocytic cup into the phagosomes (Cox, Tseng et al. 1999) and for tyrosine kinase activation and actin polymerization. Inhibition of PI3K blocks phagocytosis of IgG opsonized particles suggesting that $\mathrm{P} 13 \mathrm{~K}$ is engaged in Fc $\gamma \mathrm{R}$-mediated phagocytosis (Araki, Johnson et al. 1996). Recruitment of dynamin and vesicle associated membrane protein 3 (VAMP3) at the phagocytic cup depends on PI3K (Gold, Underhill et al. 1999). Several studies have demonstrated that PI3K participates in the control of the assembly of myosin X during phagocytosis, which includes the localization of myosin $\mathrm{X}$ within the phagocytic cup as well as the phagosome (Stendahl, Hartwig et al. 1980; Allen and Aderem 1995; Swanson, Johnson et al. 1999; Cox, Berg et al. 2002). Phosphorylation of PI to phosphatidyl inositol 3 phosphate (PI3P) by type III PI3K is necessary for phagosome maturation and NADPH oxidation (Gillooly, Simonsen et al. 2001; Kanai, Liu et al. 2001). An important point was assessed by Cox and others who showed that the effectiveness of the inhibition of phagocytosis by PI3K inhibitors is proportional to the particle size (Cox, Tseng et al. 1999). Because a greater surface is required to internalize larger particles, it has been suggested that PI3K may also be required for focal exocytosis during phagocytosis. In fact, many studies have reported a significant increase in the total surface area of macrophages during phagocytosis (Hackam, Rotstein et al. 1998; Holevinsky and Nelson 1998; Bajno, Peng et al. 2000). Transient accumulation of phospholipids like PI(4,5)P2 
and PIP3 as well as PI(4)P 5-kinase at the phagocytic cup suggested a potential role for them in focal exocytosis (Botelho, Teruel et al. 2000; Marshall, Booth et al. 2001). It has been shown that there is an accumulation of PIP3 around the phagocytic cup during pseudopodal extension just before the closure of the phagosome (Marshall, Booth et al. 2001). The accumulation of $\mathrm{PI}(3,4) \mathrm{P} 2$ could be found in the inner leaflet of plasma membrane of resting phagocytes, but it increases drastically in concentration during phagocytic cup formation and subsequently disappears leading to actin assembly and disassembly (Botelho, Teruel et al. 2000). Together PI(3,4)P2 and PIP3 regulate binding of myosin I to the plasma membrane (Adams and Pollard 1989). It has also been demonstrated that myosin's Pleckstrin homology (PH) domain binds to the PIP3 allowing its recruitment at the phagocytic site (Cox, Berg et al. 2002). The process of PI3K mediated phosphorylation of PI $(4,5)$ P2 to PIP3 is associated with increase in activities of guanine nucleotide exchange factors (GEFs) like T-cell lymphoma invasion and metastasis 1(Tiam1), Vav1 (Rho guanine nucleotide exchange factor Vav, designated Vav1, after the sixth letter of the Hebrew alphabet) and ARF nucleotide binding site opener (ARNO) (Venkateswarlu and Cullen 2000; Baumeister, Martinu et al. 2003). Also, it has been shown that the products that derive from $\mathrm{PI}(4,5) \mathrm{P} 2$ hydrolysis control transient elevation of cytosolic $\mathrm{Ca}^{+2}$ and Protein Kinase $\mathrm{C}$ (PKC) activation during phagocytosis (Zheleznyak and Brown 1992; Oancea and Meyer 1998). Protein Kinase C family isoforms are involved in different aspects of phagocytosis (Larsen, DiGennaro et al. 2000). Protein Kinase $\mathrm{C} \alpha$ is involved in FcyR-mediated phagocytosis in macrophages (Swanson and Hoppe 2004). In Fc $\gamma \mathrm{RI}$, PKC activity increases in $\mathrm{Ca}^{+2}$ independent manner (Lamberts, Johnsen et al. 2008). A PKC-dependent MAPK-activation pathway 
has been detected in mouse macrophage, monocytes and neutrophils (Garcia-Garcia and Rosales 2002). Myristoyl alanine rich C kinase substrate (MARCKS) is a PKC target found in phagosomes (Allen and Aderem 1996). Calcium-dependent PLA2, calciumdependent cytosolic PLA2 and calcium-independent cytosolic PLA2 mediate release of arachidonic acid from phosphatidyl choline or phosphatidylethanolamine (Lennartz, Lefkowith et al. 1993). Arachidonic acid acts as a second messenger in the regulation of phagocytosis. Inositol tri phosphate (IP3) releases $\mathrm{Ca}^{+2}$ ions from intracellular storage and PLC $\gamma$ accumulates at the site of phagocytic cup upon addition of IgG. Phospho lipase $\mathrm{C}$ uses $\mathrm{PI}(4,5) \mathrm{P} 2$ to generate diacyl glycerol (DAG), which then promotes phagocytosis by recruiting PKC (Larsen, Ueyama et al. 2002). Phospho lipase D (PLD) is another enzyme involved in Fc $\gamma \mathrm{R}$ mediated phagocytosis that uses phosphotidylcholine as a substrate and generates phosphatidic acid (PA) and choline (Garcia-Garcia and Rosales 2002). Elevated concentrations of $\mathrm{Ca}^{+2}$ around the phagosome have led to the notion that it might play an important role in the phagocytic process (Sawyer, Sullivan et al. 1985). However, IgG- mediated phagocytosis by human neutrophils is both $\mathrm{Ca}^{+2}$ dependent and independent, whereas IgG-mediated phagocytosis by monocytes is $\mathrm{Ca}^{+2}$ independent (Dale, Boxer et al. 2008). It has been suggested that the $\mathrm{Ca}^{+2}$ concentration increase is due to exit of $\mathrm{Ca}^{+2}$ from inside of the phagocyte to outside in cytosol (Lundqvist-Gustafsson, Gustafsson et al. 2000). Activated PLC and PLD help in accumulation of intracellular $\mathrm{Ca}^{+2}$ level (Nunes and Demaurex 2010). It is not clear if there is a second messenger that triggers $\mathrm{Ca}^{+2}$ surges in response to $\mathrm{Fc}$ receptor signaling. IP3 is the principal second messenger in most of the cellular systems to release $\mathrm{Ca}^{+2}$, but L-plastisin in Fc $\gamma$ RIIA, PLC in Fc $\gamma$ RI and Fc $\gamma$ RII and sphingosine-1-phosphate in FceRI 
are also reported to be involved (Garcia-Garcia and Rosales 2002). The Fc $\gamma \mathrm{R}$ receptor stimulates PKC and PI3K pathways to activate serine threonine kinase such as MAPK. The role of MAPK is not clear but it might be involved in PLA2 activation. In addition, MAPK may also modulate actin dynamics in phagocytosis as it phosphorylates myosin protein, which binds to actin providing mechanical force in actin dynamics. Small GTPases like Rac and Cdc42 of the Rho play an important role in actin reorganization in phagocytosis. Activation of Rac1 and Cdc42 can bind to p 85 subunit of PI3K inducing Factin polymerization through WASP and the Apoptosis related protein 2/3 (Apr2/3) complex (Vieira, Botelho et al. 2001). The Vav proteins are implicated to function as a GEF in phagocytosis (Caron and Hall 1998) including Fc $\gamma$ R-mediated phagocytosis (Patel, Hall et al. 2002). Studies have shown that Rac is recruited to the phagosome in an inactive state and is activated by Vav. Both Rac and Cdc42 bind to WASP in a GTP dependent manner (Rudolph, Bayer et al. 1998). Mostly WASP proteins are related to actin polymerization events. The Rac and Rho proteins control Cofilin, a protein that discourages actin polymerization via P21 protein (Cdc42/Rac)-activated kinase 1(PAK1) and LIM (acronym of the three gene products Lin-11, Isl-1, and Mec-3) domain kinase 1(LIMK1) (Sumi, Matsumoto et al. 1999). The Fc $\gamma$ R-mediated phagocytosis also needs ARF6, an ARF family GTPase, in actin polymerization and membrane recycling (Balana, Niedergang et al. 2005).

\subsubsection{Rabs and others in phagosome sorting: controllers of busy traffic}

The activities of lipid kinases and GTPases have been strongly implicated in the control of phagosomal vesicle trafficking (Gorvel, Chavrier et al. 1991). The Rab proteins are small GTPases involved with tethering and docking of vesicles to their target 
membrane and orchestrating the sequence of fusion events with endocytic compartments. Both Rab5 and Rab7 proteins has been detected in early and late phagosomes, respectively (Figure 2) (Vieira, Botelho et al. 2002). Early endosome antigen 1 (EEA1) is an effector that contains Rab5 binding domain. Simonsen and others demonstrated that EEA1 binds to the PI3K products PI3P via FYVE domain named after Fab 1 (yeast orthologue of PIKfyve), Vac1 (vesicle transport protein), and EEA1 (Simonsen, Lippe et al. 1998). Fratti and others showed that both PI3P and Rab5 affectors namely PI3K, Vacular protein sorting-34 (VPS34), and EEA1 are required for phagosomal maturation (Fratti, Backer et al. 2001). It has also been shown that inhibition of PI3P production prevents EEA1 recruitment into the phagosome and blocks its maturation (Fratti, Backer et al. 2001; Vieira, Botelho et al. 2001; Fratti, Chua et al. 2003). For Rab7 the only known effector is Rab7 interacting lysosomal protein (RILP), which mediates fusion of late phagosome with lysosomes. Rab7 interacting lysosomal protein plays a critical role in phagosome maturation by recruiting dynein-dynactin, a motor complex that displaces phagosomes along microtubules toward the juxtanuclear region (Harrison, Bucci et al. 2003). Along with Rab proteins, the role of Soluble N-ethylmaleimide-sensitive factor (NSF) Attachment protein Receptor (SNARE) protein is also being studied (Braun and Niedergang 2006; Hatsuzawa, Tamura et al. 2006). The membrane fusion event is mediated by SNARE proteins (Chen and Scheller 2001). The accumulation of V-SNARE on vesicles and T-SNARE on target membrane forms specific complex with tight connection initiates the opening of membrane fusion pore (McBride, Rybin et al. 1999). Syntaxin 13 located on recycling endosomes and syntaxin 7 on late endosomes have been related to phagosome maturation process in macrophage (Vieira, Botelho et al. 2002). 
Phagosome fusion with lysosomes takes place when lysosome-associated membrane proteins 1 and 2 (LAMP-1 and LAMP-2) are delivered to phagosomes during the maturation process. Huynh and others showed that LAMP-1 and LAMP-2 doubledeficient phagosomes acquired Rab5 and accumulated PI3P, but failed to recruit Rab7 and did not fuse with lysosomes (Huynh, Eskelinen et al. 2007). The LAMPs also might play a role in impairing the recruitment of RILP to the phagosome (Cantalupo, Alifano et al. ; Johansson, Rocha et.al. 2007).

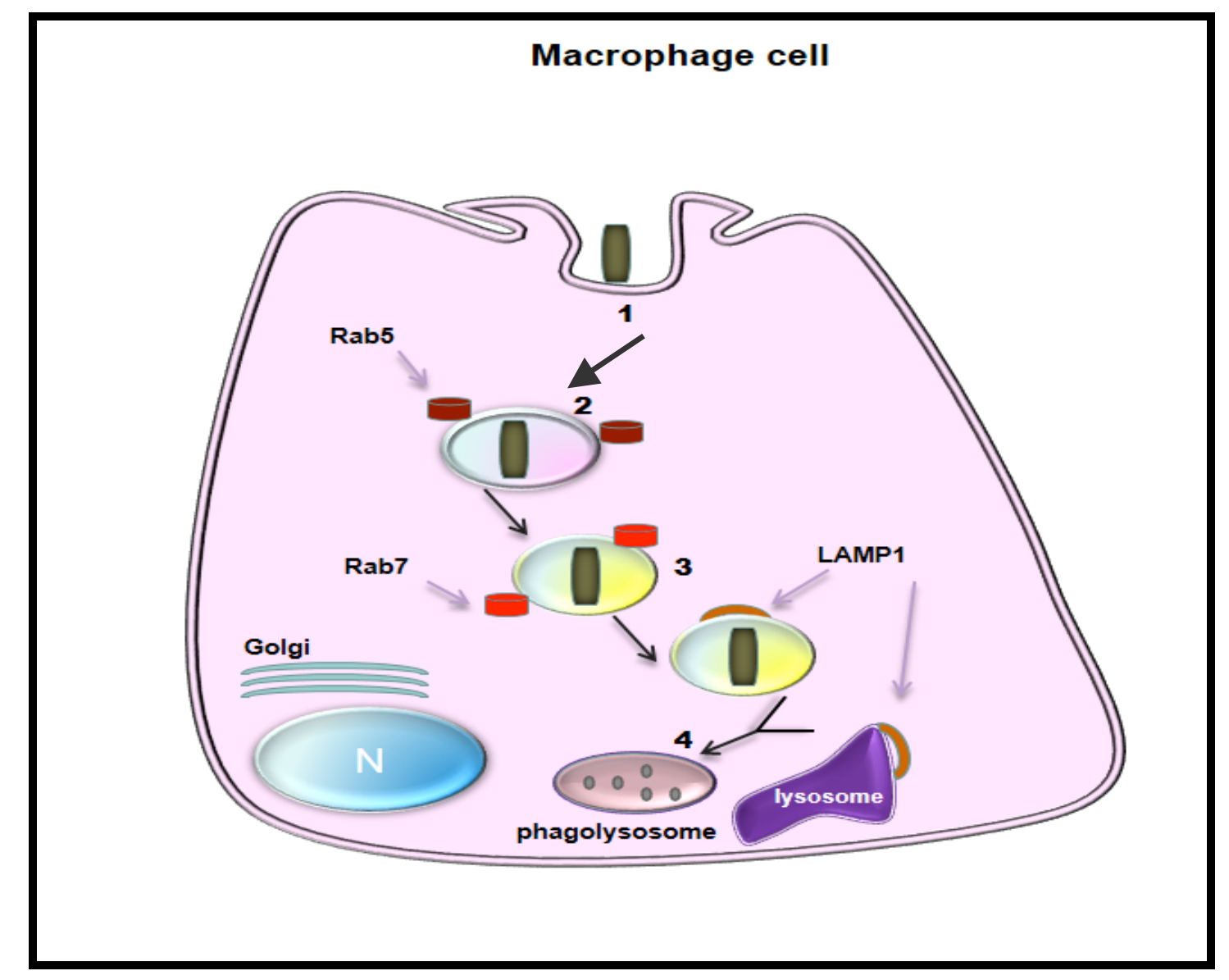

Figure 2: Phagocytic events in macrophage cell.

(1) Initiation of phagoytic cup formation and membrane ruffling. (2) Particle internalized and carried by early phagosomes with Rab5 markers on phagosomal membrane. (3) Once 
Rab7 is recruited, the phagocytic events proceed to late phase. (4) Rab7 is replaced by LAMP1 marker, signaling the fusion of late phagosome with lysosome completed by phagolysosome formation and internalized particle degradation.

\subsection{Rab5 in phagocytosis: Achill's hill?}

Cloning experiments with rat brain cDNA libraries have identified a family of GTP binding Ras-like proteins referred to as Rab proteins (Maridonneau-Parini, Yang et al. 1991). More than 60 members of Rab proteins have been classified in humans and about 11 in yeast (Pereira-Leal and Seabra 2000; Seabra, Mules et al. 2002). The Rab proteins are small GTP binding proteins involved with tethering and docking of vesicles to their target membrane and orchestrating the sequence of fusion events with other endocytic compartments. After a long cataloging of different Rabs, it has been determined that Rab1 and 2 are associated with endoplasmic reticulum, Rab3 is associated with secretory vesicles, Rab4 and 5 are endosomal, Rab6 is in the trans Golgi network, and Rab7 is in the late endosomes (Maridonneau-Parini, Yang et al. 1991). Both Rab5 and Rab7 have been reported as key regulators in maturation of phagosome (Roberts, Barbieri et al. 2000) and has been detected in early and late phagosomes, respectively (Vieira, Botelho et al. 2002). Functional studies indicate that Rab5 is a key regulator of early phagocytosis, where it is involved in clathrin-coated vesicle formation, and fusion between early phagosomes, phagosomal cargo recruitment and phagosomal motility (Duclos, 2000; Stenmark, 1994). The Rab5 protein cycles between the cytosol and the membrane of target vesicles. Once Rab5 is translated, it is associated with Rab escort proteins (REPs) (Duclos, Diez et al. 2000) and geranylated by Rab-geranylgeranyl transferase (Duclos, Diez et al. 2000). The Rab5 protein carries a GTPase fold composed 
of a six-stranded $\beta$-sheet flanked by five $\alpha$-helices which is common in all Rab structure. Downstream to GTPase fold on the $\mathrm{COOH}$ - terminus of Rab5, there is a hyper variable region followed by CAAX boxes. Covalent attachments of geranylgeranyl moieties to the cysteine residues of CAAX boxes allow the regulation of membrane insertion of Rab5 (Andres, Seabra et al. 1993; Alexandrov, Horiuchi et al. 1994; Desnoyers, Anant et al. 1996). The Rab5 protein is subsequently detached from REPs and quenched by another Rab regulating protein called GDP dissociation inhibitors (GDI). The GDI protein has the capacity to extract GDP-bound Rab5 from the membrane and delivered to the appropriate target membrane (Cavalli, Corti et al. 2001). They are inactivated upon hydrolysis of bound GTP, which converts them to their GDP-bound forms. Rab5 binds to effector proteins in their active, GTP-bound conformations. The hyper variable region shows great divergence among different phylogenetic Rab groups and this region is key determinant to specific membrane targets for Rabs. Rab5 reactivation is catalyzed by Rab5 specific GEFs that help to release Rab5 bound GDP, allowing rebinding of cytosolic GTP. The proteins that activates Rab5 possess a Vps9 (vacular protein sorting 9) domain carrying Rab5 specific GEF activity (Carney, Davies et al. 2006). Some currently established Rab5 GEFs are Rin (Ras interference) 1, Rin2, Rin3, RinL (Ras interference like protein), Rabex5 (Rabaptin 5 associated exchange factor for Rab5), Rap6 (Rab activating protein 6) (Tall, Barbieri et al. 2001; Saito, Murai et al. 2002; Kajiho, Saito et al. 2003; Hunker, Galvis et al. 2006; Delprato and Lambright 2007). Switch I and Switch II regions of Rab5 are primarily involved with nucleotide dependent Rab5 function. Both regions come in contact with the $\gamma$ phosphate of GTP during activation (Lee, Cox et al. 2000). The Rab5 protein binds to effector proteins in their 
active, GTP-bound conformations. Structural heterogeneity in the switch domains among various Rabs indicates that each type interacts with specific effector proteins to regulate their respective pathways (Pfeffer 2001; Eathiraj, Pan et al. 2005). Three isoforms of Rab5 (a, b and c) have been documented (Bucci, Lutcke et al. 1995), among which Rab5a has been found to potentiate endocytosis (Roberts, Barbieri et al. 2000). One of the earliest signs of phagosomal maturation event is the recruitment of Rab5 to phagosomes (Vieira, Bucci et al. 2003; Roberts, Chua et al. 2006; Kitano, Nakaya et al. 2008). Although a detailed mechanism for how this is accomplished during phagosome maturation is still under speculation, recent studies have implied an unexpected role for dynamin and Rabankyrin in Rab5 recruitment to the nascent phagosomes (Kinchen and Ravichandran 2008). Various Rab5 effector proteins like EEA1 (homotypic fussion between Rab5 positive vesicles), Rabenosyn (fusion between Rab5 positive vesicle to Rab7 positive vesicle), Rabaptin 5 (fusion between Rab4 positive vesicle to a Rab5 positive vesicle), share a common architecture of FYVE domain and work in conjunction with PI3P (Vitale, Rybin et al. 1998; McBride, Rybin et al. 1999; Nielsen, Christoforidis et al. 2000; Grosshans, Ortiz et al. 2006). Anomaly in Rab5 protein expression has been related to many difficult diseases (Stein, Dong et al. 2003). Benign tumors in thyroid autoimmune disease shows higher expression of Rab5 (Croizet-Berger, Daumerie et al. 2002) and both up and down regulation of Rab5 has been related to cancer (Li, Meng et al. 1999; Fukui, Tamura et al. 2007). Up regulation of Rab5 positively regulates elimination of toxic accumulation in Huntington's disease (Ravikumar, Imarisio et al. 2008). 


\subsubsection{Rab5 modulation by pathogens: escaping early death}

Microorganisms invading host tissues are first and foremost exposed to phagocytes. Bacteria that attract phagocytes and are easily ingested and killed are generally unsuccessful to establish themselves as pathogen. Whereas, successful pathogenic bacteria interfere to some extent with the activities of phagocytes or in some way avoid their attention. Microbial strategies to avoid phagocytic killing are numerous and diverse, but are usually accomplished by blocking one or of more steps in the phagocytic process such as contact between phagocyte and microbial cell, engulfment, phagosome formation, phagosome-lysosome fusion or killing and digestion (Rosenberger and Finlay 2003; Brumell and Scidmore 2007) (Figure 3). The Rab GTPases are integral to membrane traffic in eukaryotic cells. Therefore many incidences of infection accounts for modulation of Rab GTPases in host cells by pathogenic bacteria. Mycobacterium tuberculosis has adopted the role of interfering with phago-lysosome maturation process in infected macrophages arresting phagosome fusion with endosomal and lysosomal organelles (Vergne, Fratti et al. 2004). The Rab5 positive phagosomes are favorable for mycobacterial survival as they provide iron acquisition during endosome sorting (Kelley and Schorey 2004). Mycobacterium containing phagosome can retain Rab5 for 7 days post infection (p.i.) and avoid Rab7 mediated late endocytosis (Via, Deretic et al. 1997). Coxiella burnetii, the etiological agent for $\mathrm{Q}$ fever, replicates inside of a macrophage within a large vacuole. It interferes with host cell autophagic pathway to delay lysosome fusion. Early recruitment of Rab5 (5 minutes p.i. rather than typical 20 minutes p.i.) was observed in a kinetic study, where early accumulation and retention of Rab 7 was also reported (Romano, Gutierrez et al. 2007). Enterobacterial pathogen Salmonella 
typhimurium can not only escape to survive inside the host macrophage, but it can also induce apoptosis in the phagocytes (Harrison, Brumell et al. 2004). Type III secretion system in S. typhimurium promotes the accumulation of Rab5 and early Endosomal fusion (Baldeon, Ceresa et al. 2001). Thus certain pathogens have developed mechanisms to escape degradation, persisting within phagosomes by tricking Rab5 sorting and coopting the phagosome maturation machinery.

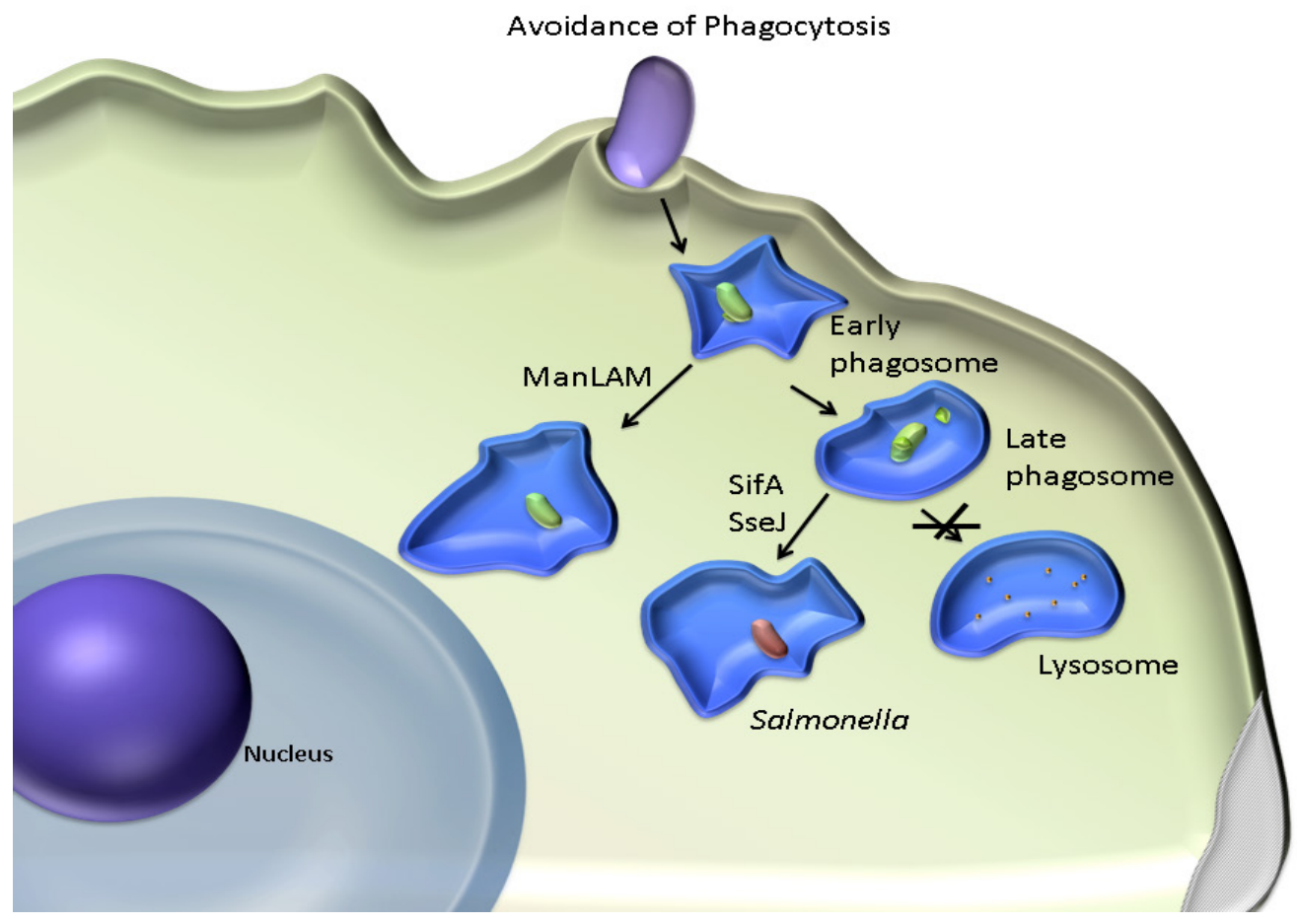

Figure 3: Pathogenic bacteria mediated subversion of phagocytosis.

Phagocytic modulation by several pathogenic bacteria such as Mycobacterium spp. and Salmonella spp. Mycobacterium spp. successfully arrests early phagosomes from maturing into late phagosomes/endosomes whereas, Salmonella spp. replicates inside arrested late phagosomes/endosomes. 


\subsection{Pseudomonas aeruginosa: the opportunist}

Pseudomonas aeruginosa is a Gram-negative bacterium belonging to the family Pseudomonadaceae and characterized as asporogenous, mono-flageletted bacillus of about 1-5 $\mu \mathrm{m}$ long and $0.5-1.0 \mu \mathrm{m}$ wide. The early reports on $P$. aeruginosa dates back to 1862 and its very first successful isolation was documented in 1882 (Lyczak, Cannon et al. 2000). Because of the dual sustenance in aerobic and anaerobic environment and ability to colonize various environmental niches, $P$. aeruginosa is ubiquitous in nature and widely found in soil, water, animals and plants (Green, Schroth et al. 1974; Falkow, Williams et al. 1976; Glazebrook, Campbell et al. 1978).

\subsubsection{Infection and Epidemiology}

Pseudomonas aeruginosa is a true opportunistic nosocomial pathogen that causes infection in immune-compromised humans taking advantage of phagocytic and immunological defects and is rarely found to cause infection in healthy individuals with strong immunity. The pathogen can thrive in any aqueous environment, soil, surface waters, sewage, plants, food, leafy vegetables and moist areas in hospital (Bonten, Bergmans et al. 1999). This notorious pathogen is a common cause of 1) secondary infections in AIDS patients, 2) bacteremia in neutropenic patients and severe burn victims in hospitals, 3) chronic lung infection in cystic fibrosis patients (Shields, Pruitt et al. 2000), 4) acute ulcerative keratitis among long-term contact lens users, 5) osteomyelitis, nerve compromise in diebetic patients, 6) medical device related infections and 7) urinary tract infection (Chitkara and Feierabend 1981; Bodey, Bolivar et al. 1983; Bendig, Kyle et al. 1987; Franzetti, Cernuschi et al. 1992; Kielhofner, Atmar et al. 1992). Pseudomonas aeruginosa also infects plants and causes damage to tomatoes, tobacco, 
onion, basil and lettuce plants (Elrod and Braun 1942). Mortality associated with $P$. aeruginosa secondary infection is higher compared to other pathogens (Trouillet, Vuagnat et al. 2002). It is a leading Gram-negative opportunistic pathogen carrying a 40$60 \%$ mortality rate at medical centers (Britigan, Hayek et al. 1993). The potent pathogen attacks up to two-thirds of the critically-ill hospitalized patients. Pseudomonas aeruginosa accounts for almost $21 \%$ cases of nosocomical pneumonia in patients (Schulert, Feltman et al. 2003). Mortality rates in Ventilator-associated pneumonia (VAP) caused by P. aeruginosa is about $34-48 \%$ (Mavrodi, Ksenzenko et al. 1998) and mortality rates in pneumonia patients with compromised immunity has been increased by 40\% (Afessa and Green 2000; Santucci, Gobara et al. 2003). According to a CDC report, $14 \%$ of $P$. aeruginosa infection in the blood stream are multi-drug resistant (Tacconelli, Tumbarello et al. 2002). It complicates $90 \%$ of cystic fibrosis deaths (Britigan, Hayek et al. 1993). As a result of antibiotic resistance, controlling P. aeruginosa infections is difficult and suitable drugs for treatment of the infections are currently limited (Endimiani, Luzzaro et al. 2006).

\subsubsection{Role of Pseudomonas aeruginosa virulence factors in immune evasion}

Pseudomonas aeruginosa expresses multiple virulence factors that contribute to its pathogenicity, help colonization and facilitate bacterial invasion. The success of this pathogen is largely because of the production of a myriad of virulence factors such as LasA protease, LasB elastase, pyoverdin, pyocyanin, and alginate as well as by biofilm formation (Figure 4). Expression of many of the virulence factors in P. aeruginosa is controlled by a quorum-sensing (QS) system. 


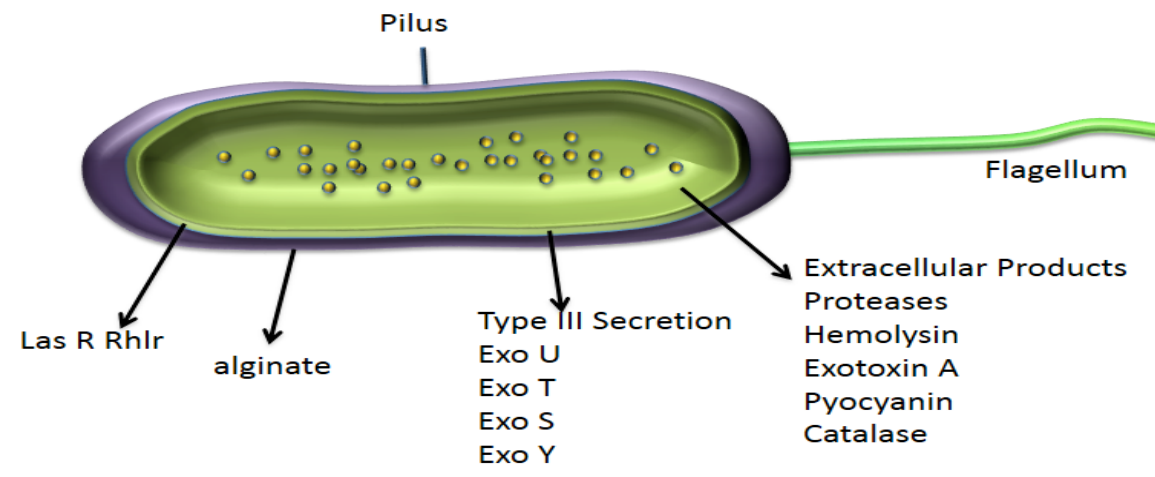

\section{Figure 4: Virulence factors of Pseudomonas aeruginosa.}

Model showing of several virulent factors in Pseudomonas aeruginosa such as surface factors includes flagellum, pilus and secreted factors, including extracellular products such as proteases such as hemolysin, exotoxin A, pyocyanin and catalases, Type III secretion proteins, transcription regulators such as las R Rhlr, and exopolysacchararides such as alginate.

\subsubsection{Surface elements}

Pseudomonas aeruginosa expresses a limited numbers of pili (Figure 4). Pili bind to eukaryotic cells, subsequently activating NF-KB and proinflammatory gene through a receptor complex that includes TLR2, and associated kinases in a lipid raft (Soong, Reddy et al. 2004). Pseudomonas aeruginosa also produces polar monotrichous flagella which are utilized mainly for motility although they seem to be involved in the initial 
stages of pulmonary infection and activate interleukin (IL)- 8 production by binding to TLR5 on the apical surface of epithelial cells (Adamo, Sokol et al. 2004). Lipopolysaccharide (LPS) is another important component of $P$. aeruginosa and other gram-negative bacteria. Environmental isolates of $P$. aeruginosa typically express smooth (typable) LPS as opposed to the strains that have adapted to Cystic Fibrosis (CF) lung, which are often nontypable and have a characteristic penta- or hexa-acylated lipid A structure which is associated with increased immunogenicity (Ernst, Yi et al. 1999). Alginate, polymer of D-mannuronic acid and L-glucuronic acid, is another extracellular polysaccharide expressed by $\mathrm{CF}$ isolates of $P$. aeruginos $a$, and is usually the consequence of a bacterial muc mutation and generally pathognomonic for $\mathrm{CF}$ isolates of $P$. aeruginosa (Boucher, Yu et al. 1997; Firoved and Deretic 2003).

\subsubsection{Type III secretion system (T3SS)}

Type III secretuin system in Gram-negative bacteria helps to secrete and inject toxic effector proteins into cytoplasm of eukaryotic host cells. The T3S apparatus is conserved between distantly related pathogenic species, but the secreted proteins are completely different in each species. Hence only one secretion mechanism can give rise to a multitude of diseases. Yersinia spp., Shigella spp., Chlamydia spp., E. coli and some plant bacteria prevalently use their T3SS to break down the phagocytosis mediated defense and make their way inside to proliferate (Hueck 1998). The T3SS complex consists of three components: the secretion apparatus, the translocation apparatus, and the secreted toxins (effector proteins) and cognate chaperones (Gauthier, Thomas et al. 2003). This system is activated on contact with eukaryotic cell membranes. PopB, PopD 
and PcrV proteins in $P$. aeruginosa facilitate the translocation of $\mathrm{T} 3 \mathrm{~S}$ effectors across host cell membrane barrier (Coburn, Sekirov et al. 2007). The secreted molecules interfere with the host signal transduction and initiate a biochemical cross talk between pathogen and host cells eventually resulting in cell death or alterations in host immune responses. Four major Type III Exoenzyme (Exo) proteins have been identified in $P$. aeruginosa (Figure 5). They are ExoS, ExoT, ExoU, and ExoY. Together they contribute to colonization, invasion and cytotoxity (Hauser 2009). The Type III secretion (T3S) effector ExoS is a bifunctional protein possessing an N-terminal Rho-GTPase-activating (Rho-GAP) domain and a C-terminal adenosine diphosphate (ADP)-ribosyltransferase (ADPr) domain, shown to induce tumor necrosis factor $\alpha$ (TNF- $\alpha$ ) production via MyD88-dependent pathway through activation of both TLR2 and TLR4 (Epelman, Stack et al. 2004). The T3S effector ExoT is also a bi-functional cytotoxin with $76 \%$ homology to ExoS. The T3S effector ExoT only shows $0.2 \%$ of its activity in vitro compared to ExoS (Barbieri and Sun 2004; Shaver and Hauser 2004; Vance, Rietsch et al. 2005; Deng and Barbieri 2008). Both ExoS and ExoT modulates host cell and disrupt internalization (Shaver and Hauser 2004; Sun, Karmakar et al. 2012). The T3S effector ExoU has been characterized as a necrotizing toxin with phospholipase activity (Sato, Frank et al. 2003) and it thereby blocked phagocyte-mediated clearance of $P$. aeruginosa upon establishment of infection (Diaz, Shaver et al. 2008). Exoenzyme Y is an adenylate cyclase and it facilitates cytoskeleton remodeling and elevated Cyclic Adenosine Mono Phosphate (cAMP) levels in cultured mammalian cells (Yahr, Vallis et al. 1998). Exoenzyme Y did not appear to play any significant role in $P$. aeruginosa pathogenesis (Shaver and Hauser 2004). The T3SS is integral to clinical disease elicited by $P$. 
aeruginosa (Hauser, Cobb et al. 2002). Several small GTPases such as Rho, Rac, Cdc42, Ras and adopter protein Crk (CT10-regulator of kinase) are found to be targeted by T3S effectors (Deng and Barbieri 2008) suggesting P. aeruginosa mediated disruption of host cell trafficking. The prevalence of the T3S phenotype was found to be significantly higher in acutely infected patients rather than in chronically infected patients with $\mathrm{CF}$ (Roy-Burman, Savel et al. 2001). Also in patients with Ventilator-Associated Pneumonia (VAP), type III-secreting isolates were associated with severe clinical outcomes (Hauser, Cobb et al. 2002), implying the importance of the secretion system in establishing $P$. aeruginosa virulence. 


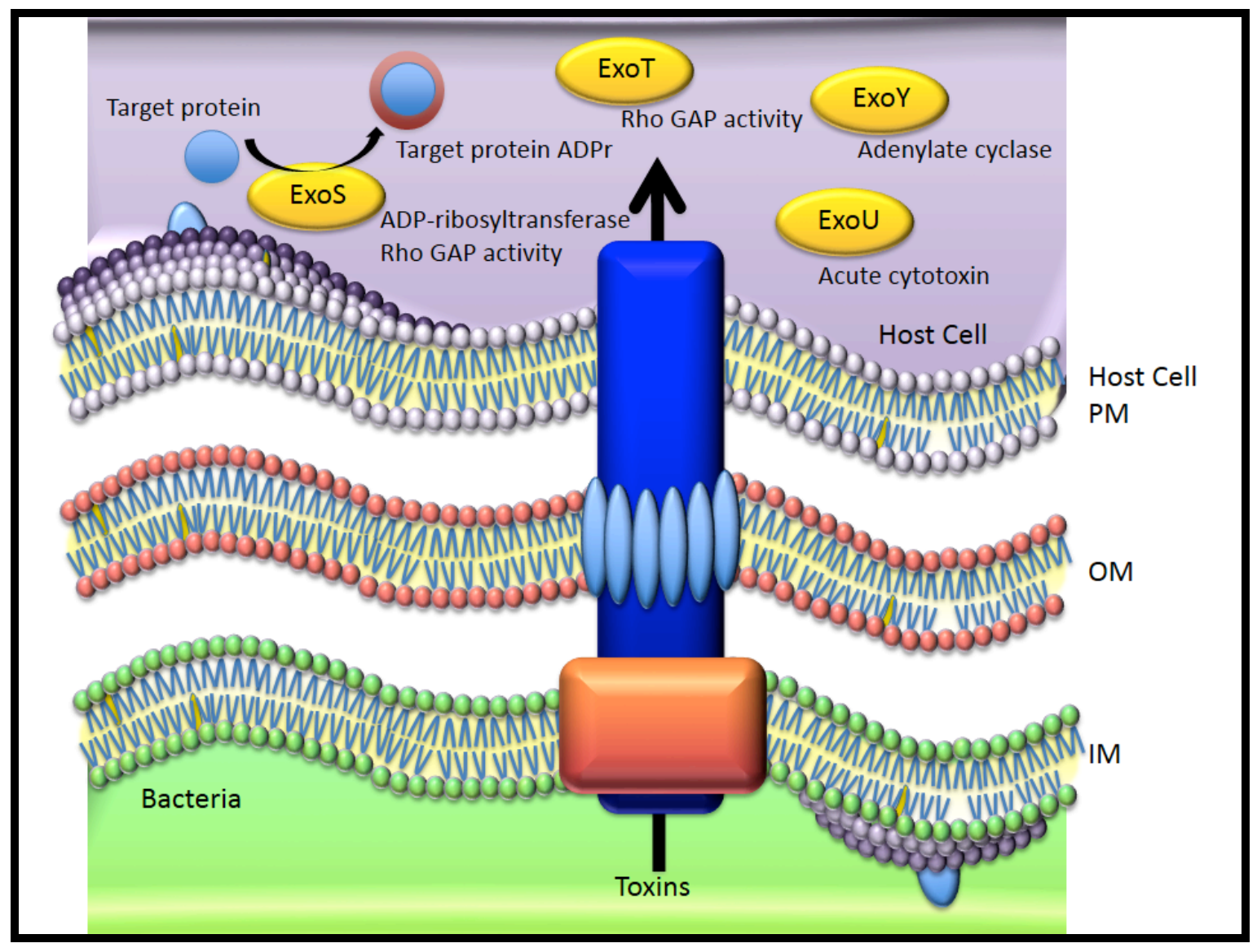

Figure 5: Schematic representations of Pseudomonas aeruginosa Type III secretion apparatus.

Type III secretion apparatus injects Type III effectors into host cells. ExoS, ExoY, ExoT and ExoU are carried from the bacterial cell and delivered into host cell via a needle like apparatus. Once inside host cell, ExoU, a phospholipase, shows acute cytotoxic effects, ExoY acts as an adenylate cyclase, ExoS and ExoT target and modulate activities of several small GTPases.

\subsubsection{Quorum sensing}

The quorum-sensing systems control expression of genes important for adaptation to the environment by a complex regulatory circuit and cell-to-cell signaling via small diffusible molecules called autoinducers (Passador, Cook et al. 1993; Davies, Parsek et 
al. 1998; Fuqua and Greenberg 2002). Pseudomonas aeruginosa produces two autoinducers: $N$-3-oxododecanoyl homoserine lactone (3-O-C12-HSL, also called PAI-1) and $N$-butyryl-L-homoserine lactone (C4- HSL, also called PAI-2) (Fuqua and Greenberg 2002; Smith and Iglewski 2003). The activation of the quorum-sensing regulates the formation of biofilms which makes conditions more favorable for bacterial persistence at the site of infection and colonization. Bacteria in biofilms are stubborn and more difficult to eradicate than those in the planktonic form (Drenkard and Ausubel 2002; Lesprit, Faurisson et al. 2003). Quorum-sensing molecules have the potential to directly modulate the host immune system (Smith, Harris et al. 2002). Quorum-sensing molecules have also shown to increase the production of inflammatory cytokines from airway cells (Smith, Fedyk et al. 2001; Smith, Kelly et al. 2002) and macrophages (Telford, Wheeler et al. 1998).

\subsubsection{Iron scavenging}

Pseudomonas aeruginosa produces two major siderophores (pyochelin and pyoverdin) that bind iron efficiently (Xiao and Kisaalita 1997) and are then taken up by the bacteria through specific cell-surface receptors to support metabolic processes and control expression of other virulence factors (Meyer, Neely et al. 1996; Vasil and Ochsner 1999; Takase, Nitanai et al. 2000; Lamont, Beare et al. 2002).

\subsubsection{Other virulence factors}

The production of elastase, alkaline proteases, hemolysins (phospholipase and lecithinase), cytotoxin (leukocidin) and diffusible pyocyanin pigments promotes tissue invasion. Pseudomonas aeruginosa elastases cleave collagen, IgG, IgA, complement, disrupt the integrity of the epithelial as well as degrade surfactant proteins A and D (SP- 
A and SP-D) (Mariencheck, Alcorn et al. 2003). Alkaline proteases inactivate important host defense proteins, such as antibodies, complement, IFN- $\alpha$, and cytokines. Phospholipase and lecithinase are hemolysins that break down lipids and lecithin and promote invasion by causing cytotoxic effects on host cells (Wilson, Pitt et al. 1987). Most $P$. aeruginosa strains secrete pyocyanin (N-methyl-1-hydroxyphenazine), the pigment that gives blue-green color to the bacterial colonies (Denning, Wollenweber et al. 1998). The ADP-ribosylating enzyme Exotoxin A enters eukaryotic cells by receptormediated endocytosis and catalyzes the ADP-ribosylation of eukaryotic elongation factor-2, consequently inhibiting protein synthesis leading to cellular death (Beattie and Merrill 1996; Yates and Merrill 2001). Exotoxin A is highly lethal in animal models of infection.

\subsection{Phagocytosis of Pseudomonas aeruginosa}

The protective mechanical barrier of skin and tissue easily controls infection in healthy individuals. Once the protective skin barrier is breached, it is convenient for the infectious pathogens to enter the blood stream. Healthy humoral response tackles intrusive pathogens at a low accumulation level of infection. The resident-mononuclear phagocytes provide the first line of defense in both the innate and adaptive immune responses to infection. In response to $P$. aeruginosa infection, macrophages have the capacity to ingest bacteria and produce inflammatory mediators that are important for host defense. $P$. aeruginosa is ingested by macrophages in a unique two-step glucose dependent process (Speert and Gordon 1992). Depletion of lung macrophages resulted in decreased bacterial clearance at 48 hours, suggesting that macrophages are important for the coordinated innate immune response to $P$. aeruginosa infection (Kooguchi, 
Hashimoto et al. 1998). Macrophages become activated when microbial products bind to cell-surface receptors including a variety of Fc receptors, complement receptor 1 and 3, macrophage mannose receptor and TLRs (Speert, Wright et al. 1988; Aderem and Ulevitch 2000). Phagocytic cells, such as macrophages, neutrophils, and dendritic cells, exhibit the expression of the highest levels of TLRs. The TLRs initiate a signal transduction cascade that results in the activation of several intracellular pathways, leading to activation of MAP kinases, NF-KB (Schultz, Knapp et al. 2003). Several receptors of TLR family interact with surface components of $P$. aeruginosa. TLR2 is engaged by pili but it may also interact with non-pilus adhesins (Lorenz, Chemotti et al. 2004). P. aeruginosa flagella initiates signaling through TLR5 and TLR2 (Hauser, Cobb et al. 2002; Madrazo, Tranguch et al. 2003) and ExoS of P. aeruginosa may activate monocytes by binding to both TLR2 and TLR4 (Epelman, Stack et al. 2004). In addition, P. aeruginosa LPS moiety has been shown to signal through TLR4 (Roger, David et al. 2001; Hajjar, Ernst et al. 2002; Backhed, Normark et al. 2003). The role of myeloid differentiation factor 88 (MyD88), which is one of the key adaptor proteins used by TLRs, is integral to the initiation of cytokine and inflammatory responses after $P$. aeruginosa infection. A recent study confirmed that MyD88-deficient mice have impaired clearance of $P$. aeruginosa (Power, Peng et al. 2004). The phagocytic response against $P$. aeruginosa also requires the small GTPases Rac1 and Cdc42 (Lee, Cox et al. 2000). Down regulation of flagellar expression has been co-related with escape from phagocytosis and TLR5-driven inflammatory signaling and hence as a survival advantage on $P$. aeruginosa colonization (Mahenthiralingam and Speert 1995). The human complement system plays an important role in clearance of pulmonary $P$. aeruginosa 
infection during early infection period when bacteria are in contact with the body fluids (Gross, Rehm et al. 1978). Deposition of the complement component C3b at the bacterial surface induces host responses (Younger, Shankar-Sinha et al. 2003). Pseudomonas. aeruginosa secretes catalytic enzymes such as alkaline protease and elastase, which degrade the complement activation (Hong and Ghebrehiwet 1992; Schmidtchen, Holst et al. 2003) and expresses LPS variants that interfere with C3b deposition at the surface (Engels, Endert et al. 1985; Schiller and Joiner 1986). An understanding of the specific pathways downstream of individual receptors will provide insights into mechanisms involved in the pathogenesis of bacterial infection and may even help develop new therapies for immuno-modulation.

\subsection{Antibiotic resistance in Pseudomonas aeruginosa: strategy to overcome the challenge}

The emergence of antibiotic resistance among virulent strains of $P$. aeruginosa is a huge impediment in current medical treatment for infected patients. There are limited numbers of reliable antimicrobial agents against $P$. aeruginosa which includes penicillins, cephalosporins, carbapenems and particularly ciprofloxacin, a second generation fluoroquinolones (Carmeli, Troillet et al. 1999). Often these drugs are administered in combination with aminoglycosides as a regimen for treatment of serious pseudomonal infections. Unfortunately, for each of these agents, emergence of resistance, causing treatment failure has been described during therapy (Quinn, Dudek et al. 1986; Milatovic and Braveny 1987; Pechere and Vladoianu 1992; Cometta, Baumgartner et al. 1994; Fink, Snydman et al. 1994). Clinically significant antibiotic resistance has arisen against virtually every antibiotic developed (Clatworthy, Pierson et al. 2007). Recently, 
many scientists are focusing on therapeutics with alternative approaches which target functional pathways of infection, such as virulence factors required to cause host damage and disease (Clatworthy, Pierson et al. 2007). Pseudomonas aeruginosa mutants, lacking type IV pili or flagellum, were shown to be seriously deficient in causing infections (Tang, Kays et al. 1995; Feldman, Bryan et al. 1998). Therefore, antimicrobials that can resist pathogenic invasion or establishment of pathogenecity could be highly beneficial and the infection could be cleared by the host immune response with little to no side effect on the host. An advantage of new antivirulence compounds is that it may impose weaker selective pressure for the development of resistance compared to the conventional antibiotics (Clatworthy, Pierson et al. 2007).

\subsection{Thesis overview}

The previous background leads to the rationale for this thesis that molecules, which

orchestrate and regulate phagosomal membrane traffic, are most likely targeted by invading pathogens. Pseudomonas aeruginosa, an important opportunistic human pathogen, displays a complex pathogenic interaction with immune compromised host. The knowledge on $P$. aeruginosa-host interaction is still poorly understood and holds high interest among microbiologists. The advancement of knowledge about detailed mechanism adopted by this pathogen to avoid host defense will be critical for drug design in the future. The previous studies have not only determined the roles of T3SS in the pathogenesis during bacterial infections, but also elucidated their potential to target and modify host proteins. However, the mechanism of regulation of host phagocytosis remains elusive and far from clear. 
The first part of this study attempts to address the crucial question, whether membrane proteins of early phagosomes are targetted by Type III secretion effectors and modified or impaired.

The aims for the first part of the dissertation are:

(I) Identify the early phagosomal membrane target(s) of $P$. aeruginosa Type III secretion system.

(II) Elucidate the molecular mechanisms of the modification.

In the second part of this study, a general investigation has been applied to imply antimicrobial properties of a natural compound Dehydroleucodine (DhL) against $P$. aeruginosa pathogenecity. Constant evolution of $P$. aeruginosa genome developing antibiotic resistance has created a demand for antimicrobials with a novel mode of mechanism.

The aim for the second part of the dissertation is:

(I) To investigate the mode of antimicrobial property of $\mathrm{DhL}$ against $P$. aeruginosa virulence factors.

This thesis is divided into 6 chapters.

Chapter 1 is the introduction and literature review. Partial content of this chapter has been published.

Chapter 2 elucidates the importance of Rab5, early phagosomal membrane protein, as a potential target of ExoS. The Rab5 activity was shown to be compromised at a very early stage of phagocytosis. Further analysis revealed that in vivo Rab5 protein is a target of ADP-ribosyl transferase activity of ExoS.This chapter has been published 
Chapter 3 reveals the effect of ADP-ribosylation of Rab5 in vitro. Rab5-GTP and Rab5GDP both could be targeted and modified by ADP-ribosylation. This study also reveals an important aspect, that more than one arginine on Rab5 protein could be target of ADPribosylation in vitro.

Chapter 4 focuses on Rab5 GEF Rin1. Interestingly, Rin1 is a Rab5 GEF as well as Ras effector protein. When over-expressed in macrophage cells Rin1 could sequester the impaired phagocytic activity and partially establish Rab5 activity during P. aeruginosa infection. Ras mediated Rin1 activity as a Rab5 GEF showed beneficial effects on macrophage activity in early phagocytic stages.

Chapter $\mathbf{5}$ is dedicated to investigating the antivirulence and antimicrobial activity in natural plant product DhL. A promising report was generated showing antivirulent activity of DhL against $P$. aeruginosa T3SS, biofilm formation and growth.

Chapter 6 summarizes the relevance and significance of the above research with future possibilities resulting from this study. 


\subsection{Reference}

Adamo, R., S. Sokol, G. Soong, M. I. Gomez and A. Prince (2004). "Pseudomonas aeruginosa flagella activate airway epithelial cells through asialoGM1 and toll-like receptor 2 as well as toll-like receptor 5." Am J Respir Cell Mol Biol 30(5): 627-634.

Adams, R. J. and T. D. Pollard (1989). "Binding of myosin I to membrane lipids." Nature 340(6234): 565-568.

Aderem, A. and R. J. Ulevitch (2000). "Toll-like receptors in the induction of the innate immune response." Nature 406(6797): 782-787.

Aderem, A. and D. M. Underhill (1999). "Mechanisms of phagocytosis in macrophages." Annu Rev Immunol 17: 593-623.

Afessa, B. and B. Green (2000). "Bacterial pneumonia in hospitalized patients with HIV infection: the Pulmonary Complications, ICU Support, and Prognostic Factors of Hospitalized Patients with HIV (PIP) Study." Chest 117(4): 1017-1022.

Akira, S. (2001). "Toll-like receptors and innate immunity." Adv Immunol 78: 1-56.

Akira, S. (2003). "Toll-like receptor signaling." J Biol Chem 278(40): 38105-38108.

Akira, S., S. Uematsu and O. Takeuchi (2006). "Pathogen recognition and innate immunity." Cell 124(4): 783-801.

Alexandrov, K., H. Horiuchi, O. Steele-Mortimer, M. C. Seabra and M. Zerial (1994). "Rab escort protein-1 is a multifunctional protein that accompanies newly prenylated rab proteins to their target membranes." EMBO J 13(22): 5262-5273.

Allen, L. A. and A. Aderem (1996). "Molecular definition of distinct cytoskeletal structures involved in complement- and Fc receptor-mediated phagocytosis in macrophages." J Exp Med 184(2): 627-637.

Allen, L. H. and A. Aderem (1995). "A role for MARCKS, the alpha isozyme of protein kinase $\mathrm{C}$ and myosin I in zymosan phagocytosis by macrophages." J Exp Med 182(3): 829-840.

Anderson, C. L., L. Shen, D. M. Eicher, M. D. Wewers and J. K. Gill (1990). "Phagocytosis mediated by three distinct Fc gamma receptor classes on human leukocytes." J Exp Med 171(4): 1333-1345.

Andres, D. A., M. C. Seabra, M. S. Brown, S. A. Armstrong, T. E. Smeland, F. P. Cremers and J. L. Goldstein (1993). "cDNA cloning of component A of Rab geranylgeranyl transferase and demonstration of its role as a Rab escort protein." Cell 73(6): 1091-1099. 
Angus, A. A., D. J. Evans, J. T. Barbieri and S. M. Fleiszig (2010). "The ADPribosylation domain of Pseudomonas aeruginosa ExoS is required for membrane bleb niche formation and bacterial survival within epithelial cells." Infect Immun 78(11): 4500-4510.

Araki, N., M. T. Johnson and J. A. Swanson (1996). "A role for phosphoinositide 3kinase in the completion of macropinocytosis and phagocytosis by macrophages." $\mathrm{J}$ Cell Biol 135(5): 1249-1260.

Backhed, F., S. Normark, E. K. Schweda, S. Oscarson and A. Richter-Dahlfors (2003). "Structural requirements for TLR4-mediated LPS signalling: a biological role for LPS modifications." Microbes Infect 5(12): 1057-1063.

Bajno, L., X. R. Peng, A. D. Schreiber, H. P. Moore, W. S. Trimble and S. Grinstein (2000). "Focal exocytosis of VAMP3-containing vesicles at sites of phagosome formation." J Cell Biol 149(3): 697-706.

Balana, M. E., F. Niedergang, A. Subtil, A. Alcover, P. Chavrier and A. Dautry-Varsat (2005). "ARF6 GTPase controls bacterial invasion by actin remodelling." J Cell Sci 118(Pt 10): 2201-2210.

Baldeon, M. E., B. P. Ceresa and J. E. Casanova (2001). "Expression of constitutively active Rab5 uncouples maturation of the Salmonella-containing vacuole from intracellular replication." Cell Microbiol 3(7): 473-486.

Barbieri, J. T. and J. Sun (2004). "Pseudomonas aeruginosa ExoS and ExoT." Rev Physiol Biochem Pharmacol 152: 79-92.

Baumeister, M. A., L. Martinu, K. L. Rossman, J. Sondek, M. A. Lemmon and M. M. Chou (2003). "Loss of phosphatidylinositol 3-phosphate binding by the C-terminal Tiam1 pleckstrin homology domain prevents in vivo Rac1 activation without affecting membrane targeting." J Biol Chem 278(13): 11457-11464.

Beattie, B. K. and A. R. Merrill (1996). "In vitro enzyme activation and folded stability of Pseudomonas aeruginosa exotoxin A and its C-terminal peptide." Biochemistry 35(28): 9042-9051.

Bendig, J. W., P. W. Kyle, P. L. Giangrande, D. M. Samson and B. S. Azadian (1987). "Two neutropenic patients with multiple resistant Pseudomonas aeruginosa septicaemia treated with ciprofloxacin." J R Soc Med 80(5): 316-317.

Blander, J. M. and R. Medzhitov (2006). "On regulation of phagosome maturation and antigen presentation." Nat Immunol 7(10): 1029-1035.

Bodey, G. P., R. Bolivar, V. Fainstein and L. Jadeja (1983). "Infections caused by Pseudomonas aeruginosa." Rev Infect Dis 5(2): 279-313. 
Bonten, M. J., D. C. Bergmans, H. Speijer and E. E. Stobberingh (1999). "Characteristics of polyclonal endemicity of Pseudomonas aeruginosa colonization in intensive care units. Implications for infection control." Am J Respir Crit Care Med 160(4): 1212-1219.

Botelho, R. J., D. J. Hackam, A. D. Schreiber and S. Grinstein (2000). "Role of COPI in phagosome maturation." J Biol Chem 275(21): 15717-15727.

Botelho, R. J., H. Tapper, W. Furuya, D. Mojdami and S. Grinstein (2002). "Fc gamma R-mediated phagocytosis stimulates localized pinocytosis in human neutrophils." J Immunol 169(8): 4423-4429.

Botelho, R. J., M. Teruel, R. Dierckman, R. Anderson, A. Wells, J. D. York, T. Meyer and S. Grinstein (2000). "Localized biphasic changes in phosphatidylinositol-4,5bisphosphate at sites of phagocytosis." J Cell Biol 151(7): 1353-1368.

Boucher, J. C., H. Yu, M. H. Mudd and V. Deretic (1997). "Mucoid Pseudomonas aeruginosa in cystic fibrosis: characterization of muc mutations in clinical isolates and analysis of clearance in a mouse model of respiratory infection." Infect Immun 65(9): 3838-3846.

Bozzaro, S. and L. Eichinger (2011). "The professional phagocyte Dictyostelium discoideum as a model host for bacterial pathogens." Curr Drug Targets 12(7): 942-954.

Braun, M., A. Wendt, B. Birnir, J. Broman, L. Eliasson, J. Galvanovskis, J. Gromada, H. Mulder and P. Rorsman (2004). "Regulated exocytosis of GABA-containing synapticlike microvesicles in pancreatic beta-cells." J Gen Physiol 123(3): 191-204.

Braun, V. and F. Niedergang (2006). "Linking exocytosis and endocytosis during phagocytosis." Biol Cell 98(3): 195-201.

Britigan, B. E., M. B. Hayek, B. N. Doebbeling and R. B. Fick, Jr. (1993). "Transferrin and lactoferrin undergo proteolytic cleavage in the Pseudomonas aeruginosa-infected lungs of patients with cystic fibrosis." Infect Immun 61(12): 5049-5055.

Brumell, J. H. and M. A. Scidmore (2007). "Manipulation of rab GTPase function by intracellular bacterial pathogens." Microbiol Mol Biol Rev 71(4): 636-652.

Bucci, C., A. Lutcke, O. Steele-Mortimer, V. M. Olkkonen, P. Dupree, M. Chiariello, C. B. Bruni, K. Simons and M. Zerial (1995). "Co-operative regulation of endocytosis by three Rab5 isoforms." FEBS Lett 366(1): 65-71.

Cantalupo, G., P. Alifano, V. Roberti, C. B. Bruni and C. Bucci (2001). "Rab-interacting lysosomal protein (RILP): the Rab7 effector required for transport to lysosomes." EMBO J 20(4): 683-693. 
Carmeli, Y., N. Troillet, G. M. Eliopoulos and M. H. Samore (1999). "Emergence of antibiotic-resistant Pseudomonas aeruginosa: comparison of risks associated with different antipseudomonal agents." Antimicrob Agents Chemother 43(6): 1379-1382.

Carney, D. S., B. A. Davies and B. F. Horazdovsky (2006). "Vps9 domain-containing proteins: activators of Rab5 GTPases from yeast to neurons." Trends Cell Biol 16(1): 2735 .

Caron, E. and A. Hall (1998). "Identification of two distinct mechanisms of phagocytosis controlled by different Rho GTPases." Science 282(5394): 1717-1721.

Carroll, M. C. (1998). "The role of complement and complement receptors in induction and regulation of immunity." Annu Rev Immunol 16: 545-568.

Cavalli, V., M. Corti and J. Gruenberg (2001). "Endocytosis and signaling cascades: a close encounter." FEBS Lett 498(2-3): 190-196.

Celli, J. and B. B. Finlay (2002). "Bacterial avoidance of phagocytosis." Trends Microbiol 10(5): 232-237.

Chavele, K. M., L. Martinez-Pomares, J. Domin, S. Pemberton, S. M. Haslam, A. Dell, H. T. Cook, C. D. Pusey, S. Gordon and A. D. Salama (2010). "Mannose receptor interacts with $\mathrm{Fc}$ receptors and is critical for the development of crescentic glomerulonephritis in mice." J Clin Invest 120(5): 1469-1478.

Chen, Y. A. and R. H. Scheller (2001). "SNARE-mediated membrane fusion." Nat Rev Mol Cell Biol 2(2): 98-106.

Chitkara, Y. K. and T. C. Feierabend (1981). "Endogenous and exogenous infection with Pseudomonas aeruginosa in a burns unit." Int Surg 66(3): 237-240.

Clatworthy, A. E., E. Pierson and D. T. Hung (2007). "Targeting virulence: a new paradigm for antimicrobial therapy." Nat Chem Biol 3(9): 541-548.

Coburn, B., I. Sekirov and B. B. Finlay (2007). "Type III secretion systems and disease." Clin Microbiol Rev 20(4): 535-549.

Coller, S. P. and D. M. Paulnock (2001). "Signaling pathways initiated in macrophages after engagement of type A scavenger receptors." J Leukoc Biol 70(1): 142-148.

Cometta, A., J. D. Baumgartner, D. Lew, W. Zimmerli, D. Pittet, P. Chopart, U. Schaad, C. Herter, P. Eggimann, O. Huber and et al. (1994). "Prospective randomized comparison of imipenem monotherapy with imipenem plus netilmicin for treatment of severe infections in nonneutropenic patients." Antimicrob Agents Chemother 38(6): 1309-1313. 
Cox, D., J. S. Berg, M. Cammer, J. O. Chinegwundoh, B. M. Dale, R. E. Cheney and S. Greenberg (2002). "Myosin X is a downstream effector of PI(3)K during phagocytosis." Nat Cell Biol 4(7): 469-477.

Cox, D., D. J. Lee, B. M. Dale, J. Calafat and S. Greenberg (2000). "A Rab11-containing rapidly recycling compartment in macrophages that promotes phagocytosis." Proc Natl Acad Sci U S A 97(2): 680-685.

Cox, D., C. C. Tseng, G. Bjekic and S. Greenberg (1999). "A requirement for phosphatidylinositol 3-kinase in pseudopod extension." J Biol Chem 274(3): 1240-1247.

Croizet-Berger, K., C. Daumerie, M. Couvreur, P. J. Courtoy and M. F. van den Hove (2002). "The endocytic catalysts, Rab5a and Rab7, are tandem regulators of thyroid hormone production." Proc Natl Acad Sci U S A 99(12): 8277-8282.

Crowley, M. T., P. S. Costello, C. J. Fitzer-Attas, M. Turner, F. Meng, C. Lowell, V. L. Tybulewicz and A. L. DeFranco (1997). "A critical role for Syk in signal transduction and phagocytosis mediated by Fcgamma receptors on macrophages." J Exp Med 186(7): 1027-1039.

Dale, D. C., L. Boxer and W. C. Liles (2008). "The phagocytes: neutrophils and monocytes." Blood 112(4): 935-945.

Davies, D. G., M. R. Parsek, J. P. Pearson, B. H. Iglewski, J. W. Costerton and E. P. Greenberg (1998). "The involvement of cell-to-cell signals in the development of a bacterial biofilm." Science 280(5361): 295-298.

De Camilli, P., S. D. Emr, P. S. McPherson and P. Novick (1996). "Phosphoinositides as regulators in membrane traffic." Science 271(5255): 1533-1539.

Delprato, A. and D. G. Lambright (2007). "Structural basis for Rab GTPase activation by VPS9 domain exchange factors." Nat Struct Mol Biol 14(5): 406-412.

Deng, Q. and J. T. Barbieri (2008). "Modulation of host cell endocytosis by the type III cytotoxin, Pseudomonas ExoS." Traffic 9(11): 1948-1957.

Deng, Q. and J. T. Barbieri (2008). "Molecular mechanisms of the cytotoxicity of ADPribosylating toxins." Annu Rev Microbiol 62: 271-288.

Denning, G. M., L. A. Wollenweber, M. A. Railsback, C. D. Cox, L. L. Stoll and B. E. Britigan (1998). "Pseudomonas pyocyanin increases interleukin-8 expression by human airway epithelial cells." Infect Immun 66(12): 5777-5784.

Desjardins, M., L. A. Huber, R. G. Parton and G. Griffiths (1994). "Biogenesis of phagolysosomes proceeds through a sequential series of interactions with the endocytic apparatus." J Cell Biol 124(5): 677-688. 
Desnoyers, L., J. S. Anant and M. C. Seabra (1996). "Geranylgeranylation of Rab proteins." Biochem Soc Trans 24(3): 699-703.

Diakonova, M., G. Bokoch and J. A. Swanson (2002). "Dynamics of cytoskeletal proteins during Fcgamma receptor-mediated phagocytosis in macrophages." Mol Biol Cell 13(2): 402-411.

Diaz, M. H., C. M. Shaver, J. D. King, S. Musunuri, J. A. Kazzaz and A. R. Hauser (2008). "Pseudomonas aeruginosa induces localized immunosuppression during pneumonia." Infect Immun 76(10): 4414-4421.

Drenkard, E. and F. M. Ausubel (2002). "Pseudomonas biofilm formation and antibiotic resistance are linked to phenotypic variation." Nature 416(6882): 740-743.

Duclos, S., R. Diez, J. Garin, B. Papadopoulou, A. Descoteaux, H. Stenmark and M. Desjardins (2000). "Rab5 regulates the kiss and run fusion between phagosomes and endosomes and the acquisition of phagosome leishmanicidal properties in RAW 264.7 macrophages." J Cell Sci 113 Pt 19: 3531-3541.

Eathiraj, S., X. Pan, C. Ritacco and D. G. Lambright (2005). "Structural basis of familywide Rab GTPase recognition by rabenosyn-5." Nature 436(7049): 415-419.

Elrod, R. P. and A. C. Braun (1942). "Pseudomonas aeruginosa: Its Role as a Plant Pathogen." J Bacteriol 44(6): 633-645.

Elshourbagy, N. A., X. Li, J. Terrett, S. Vanhorn, M. S. Gross, J. E. Adamou, K. M. Anderson, C. L. Webb and P. G. Lysko (2000). "Molecular characterization of a human scavenger receptor, human MARCO." Eur J Biochem 267(3): 919-926.

Endimiani, A., F. Luzzaro, B. Pini, G. Amicosante, G. M. Rossolini and A. Q. Toniolo (2006). "Pseudomonas aeruginosa bloodstream infections: risk factors and treatment outcome related to expression of the PER-1 extended-spectrum beta-lactamase." BMC Infect Dis 6: 52.

Engels, W., J. Endert, M. A. Kamps and C. P. van Boven (1985). "Role of lipopolysaccharide in opsonization and phagocytosis of Pseudomonas aeruginosa." Infect Immun 49(1): 182-189.

Epelman, S., D. Stack, C. Bell, E. Wong, G. G. Neely, S. Krutzik, K. Miyake, P. Kubes, L. D. Zbytnuik, L. L. Ma, X. Xie, D. E. Woods and C. H. Mody (2004). "Different domains of Pseudomonas aeruginosa exoenzyme S activate distinct TLRs." J Immunol 173(3): 2031-2040.

Ernst, R. K., E. C. Yi, L. Guo, K. B. Lim, J. L. Burns, M. Hackett and S. I. Miller (1999). "Specific lipopolysaccharide found in cystic fibrosis airway Pseudomonas aeruginosa." Science 286(5444): 1561-1565. 
Ezekowitz, R. A., K. Sastry, P. Bailly and A. Warner (1990). "Molecular characterization of the human macrophage mannose receptor: demonstration of multiple carbohydrate recognition-like domains and phagocytosis of yeasts in Cos-1 cells." J Exp Med 172(6): 1785-1794.

Falkow, S., L. P. Williams, Jr., S. L. Seaman and L. D. Rollins (1976). "Increased survival in calves of Esherichia coli K-12 carrying an Ent plasmid." Infect Immun 13(3): 1005-1007.

Feldman, M., R. Bryan, S. Rajan, L. Scheffler, S. Brunnert, H. Tang and A. Prince (1998). "Role of flagella in pathogenesis of Pseudomonas aeruginosa pulmonary infection." Infect Immun 66(1): 43-51.

Fink, M. P., D. R. Snydman, M. S. Niederman, K. V. Leeper, Jr., R. H. Johnson, S. O. Heard, R. G. Wunderink, J. W. Caldwell, J. J. Schentag, G. A. Siami and et al. (1994). "Treatment of severe pneumonia in hospitalized patients: results of a multicenter, randomized, double-blind trial comparing intravenous ciprofloxacin with imipenemcilastatin. The Severe Pneumonia Study Group." Antimicrob Agents Chemother 38(3): $547-557$.

Firoved, A. M. and V. Deretic (2003). "Microarray analysis of global gene expression in mucoid Pseudomonas aeruginosa." J Bacteriol 185(3): 1071-1081.

Franzetti, F., M. Cernuschi, R. Esposito and M. Moroni (1992). "Pseudomonas infections in patients with AIDS and AIDS-related complex." J Intern Med 231(4): 437-443.

Fratti, R. A., J. M. Backer, J. Gruenberg, S. Corvera and V. Deretic (2001). "Role of phosphatidylinositol 3-kinase and Rab5 effectors in phagosomal biogenesis and mycobacterial phagosome maturation arrest." J Cell Biol 154(3): 631-644.

Fratti, R. A., J. Chua and V. Deretic (2003). "Induction of p38 mitogen-activated protein kinase reduces early endosome autoantigen 1 (EEA1) recruitment to phagosomal membranes." J Biol Chem 278(47): 46961-46967.

Fukui, K., S. Tamura, A. Wada, Y. Kamada, T. Igura, S. Kiso and N. Hayashi (2007). "Expression of Rab5a in hepatocellular carcinoma: Possible involvement in epidermal growth factor signaling." Hepatol Res 37(11): 957-965.

Fuqua, C. and E. P. Greenberg (2002). "Listening in on bacteria: acyl-homoserine lactone signalling." Nat Rev Mol Cell Biol 3(9): 685-695.

Garcia-Garcia, E. and C. Rosales (2002). "Signal transduction during Fc receptormediated phagocytosis." J Leukoc Biol 72(6): 1092-1108.

Gauthier, A., N. A. Thomas and B. B. Finlay (2003). "Bacterial injection machines." J Biol Chem 278(28): 25273-25276. 
Ghazizadeh, S. and H. B. Fleit (1994). "Tyrosine phosphorylation provides an obligatory early signal for Fc gamma RII-mediated endocytosis in the monocytic cell line THP-1." J Immunol 152(1): 30-41.

Gillooly, D. J., A. Simonsen and H. Stenmark (2001). "Phosphoinositides and phagocytosis." J Cell Biol 155(1): 15-17.

Glazebrook, J. S., R. S. Campbell, G. W. Hutchinson and N. D. Stallman (1978). "Rodent zoonoses in North Queensland: the occurrence and distribution of zoonotic infections in North Queensland rodents." Aust J Exp Biol Med Sci 56(2): 147-156.

Gold, E. S., D. M. Underhill, N. S. Morrissette, J. Guo, M. A. McNiven and A. Aderem (1999). "Dynamin 2 is required for phagocytosis in macrophages." J Exp Med 190(12): 1849-1856.

Gordon, S. B., M. E. Molyneux, M. J. Boeree, S. Kanyanda, M. Chaponda, S. B. Squire and R. C. Read (2001). "Opsonic phagocytosis of Streptococcus pneumoniae by alveolar macrophages is not impaired in human immunodeficiency virus-infected Malawian adults." J Infect Dis 184(10): 1345-1349.

Gorvel, J. P., P. Chavrier, M. Zerial and J. Gruenberg (1991). "rab5 controls early endosome fusion in vitro." Cell 64(5): 915-925.

Green, S. K., M. N. Schroth, J. J. Cho, S. K. Kominos and V. B. Vitanza-jack (1974). "Agricultural plants and soil as a reservoir for Pseudomonas aeruginosa." Appl Microbiol 28(6): 987-991.

Griffin, F. M., Jr. and S. C. Silverstein (1974). "Segmental response of the macrophage plasma membrane to a phagocytic stimulus." J Exp Med 139(2): 323-336.

Gross, G. N., S. R. Rehm and A. K. Pierce (1978). "The effect of complement depletion on lung clearance of bacteria." J Clin Invest 62(2): 373-378.

Grosshans, B. L., D. Ortiz and P. Novick (2006). "Rabs and their effectors: achieving specificity in membrane traffic." Proc Natl Acad Sci U S A 103(32): 11821-11827.

Gu, H., R. J. Botelho, M. Yu, S. Grinstein and B. G. Neel (2003). "Critical role for scaffolding adapter Gab2 in Fc gamma R-mediated phagocytosis." J Cell Biol 161(6): 1151-1161.

Hackam, D. J., O. D. Rotstein, A. Schreiber, W. Zhang and S. Grinstein (1997). "Rho is required for the initiation of calcium signaling and phagocytosis by Fcgamma receptors in macrophages." J Exp Med 186(6): 955-966.

Hackam, D. J., O. D. Rotstein, C. Sjolin, A. D. Schreiber, W. S. Trimble and S. Grinstein (1998). "v-SNARE-dependent secretion is required for phagocytosis." Proc Natl Acad Sci U S A 95(20): 11691-11696. 
Hajjar, A. M., R. K. Ernst, J. H. Tsai, C. B. Wilson and S. I. Miller (2002). "Human Tolllike receptor 4 recognizes host-specific LPS modifications." Nat Immunol 3(4): 354-359.

Hallett, M. B. and S. Dewitt (2007). "Ironing out the wrinkles of neutrophil phagocytosis." Trends Cell Biol 17(5): 209-214.

Harrison, R. E., J. H. Brumell, A. Khandani, C. Bucci, C. C. Scott, X. Jiang, B. B. Finlay and S. Grinstein (2004). "Salmonella impairs RILP recruitment to Rab7 during maturation of invasion vacuoles." Mol Biol Cell 15(7): 3146-3154.

Harrison, R. E., C. Bucci, O. V. Vieira, T. A. Schroer and S. Grinstein (2003). "Phagosomes fuse with late endosomes and/or lysosomes by extension of membrane protrusions along microtubules: role of Rab7 and RILP." Mol Cell Biol 23(18): 64946506.

Hatsuzawa, K., T. Tamura, H. Hashimoto, S. Yokoya, M. Miura, H. Nagaya and I. Wada (2006). "Involvement of syntaxin 18, an endoplasmic reticulum (ER)-localized SNARE protein, in ER-mediated phagocytosis." Mol Biol Cell 17(9): 3964-3977.

Hauser, A. R. (2009). "The type III secretion system of Pseudomonas aeruginosa: infection by injection." Nat Rev Microbiol 7(9): 654-665.

Hauser, A. R., E. Cobb, M. Bodi, D. Mariscal, J. Valles, J. N. Engel and J. Rello (2002). "Type III protein secretion is associated with poor clinical outcomes in patients with ventilator-associated pneumonia caused by Pseudomonas aeruginosa." Crit Care Med 30(3): 521-528.

Henry, R. M., A. D. Hoppe, N. Joshi and J. A. Swanson (2004). "The uniformity of phagosome maturation in macrophages." J Cell Biol 164(2): 185-194.

Holevinsky, K. O. and D. J. Nelson (1998). "Membrane capacitance changes associated with particle uptake during phagocytosis in macrophages." Biophys J 75(5): 2577-2586.

Hong, Y. Q. and B. Ghebrehiwet (1992). "Effect of Pseudomonas aeruginosa elastase and alkaline protease on serum complement and isolated components $\mathrm{C} 1 \mathrm{q}$ and $\mathrm{C} 3$." Clin Immunol Immunopathol 62(2): 133-138.

Hueck, C. J. (1998). "Type III protein secretion systems in bacterial pathogens of animals and plants." Microbiol Mol Biol Rev 62(2): 379-433.

Hunker, C. M., A. Galvis, I. Kruk, H. Giambini, M. L. Veisaga and M. A. Barbieri (2006). "Rab5-activating protein 6, a novel endosomal protein with a role in endocytosis." Biochem Biophys Res Commun 340(3): 967-975.

Huynh, K. K., E. L. Eskelinen, C. C. Scott, A. Malevanets, P. Saftig and S. Grinstein (2007). "LAMP proteins are required for fusion of lysosomes with phagosomes." EMBO J 26(2): 313-324. 
Izadi, K. D., A. Erdreich-Epstein, Y. Liu and D. L. Durden (1998). "Characterization of Cbl-Nck and Nck-Pak1 interactions in myeloid FcgammaRII signaling." Exp Cell Res 245(2): 330-342.

Janeway, C. A., Jr. (1989). "Approaching the asymptote? Evolution and revolution in immunology." Cold Spring Harb Symp Quant Biol 54 Pt 1: 1-13.

Janeway, C. A., Jr. and R. Medzhitov (2002). "Innate immune recognition." Annu Rev Immunol 20: 197-216.

Jiang, K., B. Zhong, D. L. Gilvary, B. C. Corliss, E. Vivier, E. Hong-Geller, S. Wei and J. Y. Djeu (2002). "Syk regulation of phosphoinositide 3-kinase-dependent NK cell function." J Immunol 168(7): 3155-3164.

Johansson, M., N. Rocha, W. Zwart, I. Jordens, L. Janssen, C. Kuijl, V. M. Olkkonen and J. Neefjes (2007). "Activation of endosomal dynein motors by stepwise assembly of Rab7-RILP-p150Glued, ORP1L, and the receptor betalll spectrin." J Cell Biol 176(4): 459-471.

Jones, D. H., J. Nusbacher and C. L. Anderson (1985). "Fc receptor-mediated binding and endocytosis by human mononuclear phagocytes: monomeric $\mathrm{IgG}$ is not endocytosed by U937 cells and monocytes." J Cell Biol 100(2): 558-564.

Kajiho, H., K. Saito, K. Tsujita, K. Kontani, Y. Araki, H. Kurosu and T. Katada (2003). "RIN3: a novel Rab5 GEF interacting with amphiphysin II involved in the early endocytic pathway." J Cell Sci 116(Pt 20): 4159-4168.

Kanai, F., H. Liu, S. J. Field, H. Akbary, T. Matsuo, G. E. Brown, L. C. Cantley and M. B. Yaffe (2001). "The PX domains of p47phox and p40phox bind to lipid products of PI(3)K." Nat Cell Biol 3(7): 675-678.

Kavran, J. M., D. E. Klein, A. Lee, M. Falasca, S. J. Isakoff, E. Y. Skolnik and M. A. Lemmon (1998). "Specificity and promiscuity in phosphoinositide binding by pleckstrin homology domains." J Biol Chem 273(46): 30497-30508.

Kelley, V. A. and J. S. Schorey (2004). "Modulation of cellular phosphatidylinositol 3phosphate levels in primary macrophages affects heat-killed but not viable Mycobacterium avium's transport through the phagosome maturation process." Cell Microbiol 6(10): 973-985.

Kiefer, F., J. Brumell, N. Al-Alawi, S. Latour, A. Cheng, A. Veillette, S. Grinstein and T. Pawson (1998). "The Syk protein tyrosine kinase is essential for Fcgamma receptor signaling in macrophages and neutrophils." Mol Cell Biol 18(7): 4209-4220.

Kielhofner, M., R. L. Atmar, R. J. Hamill and D. M. Musher (1992). "Life-threatening Pseudomonas aeruginosa infections in patients with human immunodeficiency virus infection." Clin Infect Dis 14(2): 403-411. 
Kiener, P. A., B. M. Rankin, A. L. Burkhardt, G. L. Schieven, L. K. Gilliland, R. B. Rowley, J. B. Bolen and J. A. Ledbetter (1993). "Cross-linking of Fc gamma receptor I (Fc gamma RI) and receptor II (Fc gamma RII) on monocytic cells activates a signal transduction pathway common to both $\mathrm{Fc}$ receptors that involves the stimulation of $\mathrm{p} 72$ Syk protein tyrosine kinase." J Biol Chem 268(32): 24442-24448.

Kinchen, J. M., K. Doukoumetzidis, J. Almendinger, L. Stergiou, A. Tosello-Trampont, C. D. Sifri, M. O. Hengartner and K. S. Ravichandran (2008). "A pathway for phagosome maturation during engulfment of apoptotic cells." Nat Cell Biol 10(5): 556-566.

Kinchen, J. M. and K. S. Ravichandran (2008). "Phagosome maturation: going through the acid test." Nat Rev Mol Cell Biol 9(10): 781-795.

Kitano, M., M. Nakaya, T. Nakamura, S. Nagata and M. Matsuda (2008). "Imaging of Rab5 activity identifies essential regulators for phagosome maturation." Nature 453(7192): 241-245.

Kodama, T., T. Doi, H. Suzuki, K. Takahashi, Y. Wada and S. Gordon (1996). "Collagenous macrophage scavenger receptors." Curr Opin Lipidol 7(5): 287-291.

Kooguchi, K., S. Hashimoto, A. Kobayashi, Y. Kitamura, I. Kudoh, J. Wiener-Kronish and T. Sawa (1998). "Role of alveolar macrophages in initiation and regulation of inflammation in Pseudomonas aeruginosa pneumonia." Infect Immun 66(7): 3164-3169.

Kwiatkowska, K., J. Frey and A. Sobota (2003). "Phosphorylation of FegammaRIIA is required for the receptor-induced actin rearrangement and capping: the role of membrane rafts." J Cell Sci 116(Pt 3): 537-550.

Kwiatkowska, K. and A. Sobota (1999). "Signaling pathways in phagocytosis." Bioessays 21(5): 422-431.

Lamberts, R. F., A. R. Johnsen, O. Andersen and J. H. Christensen (2008). "Univariate and multivariate characterization of heavy fuel oil weathering and biodegradation in soil." Environ Pollut 156(2): 297-305.

Lamont, I. L., P. A. Beare, U. Ochsner, A. I. Vasil and M. L. Vasil (2002). "Siderophoremediated signaling regulates virulence factor production in Pseudomonasaeruginosa." Proc Natl Acad Sci U S A 99(10): 7072-7077.

Larsen, E. C., J. A. DiGennaro, N. Saito, S. Mehta, D. J. Loegering, J. E. Mazurkiewicz and M. R. Lennartz (2000). "Differential requirement for classic and novel PKC isoforms in respiratory burst and phagocytosis in RAW 264.7 cells." J Immunol 165(5): 28092817.

Larsen, E. C., T. Ueyama, P. M. Brannock, Y. Shirai, N. Saito, C. Larsson, D. Loegering, P. B. Weber and M. R. Lennartz (2002). "A role for PKC-epsilon in Fc gammaRmediated phagocytosis by RAW 264.7 cells." J Cell Biol 159(6): 939-944. 
Larsson, M., J. F. Fonteneau and N. Bhardwaj (2001). "Dendritic cells resurrect antigens from dead cells." Trends Immunol 22(3): 141-148.

Lee, D. J., D. Cox, J. Li and S. Greenberg (2000). "Rac1 and Cdc42 are required for phagocytosis, but not NF-kappaB-dependent gene expression, in macrophages challenged with Pseudomonas aeruginosa." J Biol Chem 275(1): 141-146.

Lee, W. L., G. Cosio, K. Ireton and S. Grinstein (2007). "Role of CrkII in Fegamma receptor-mediated phagocytosis." J Biol Chem 282(15): 11135-11143.

Lennartz, M. R. (1999). "Phospholipases and phagocytosis: the role of phospholipidderived second messengers in phagocytosis." Int J Biochem Cell Biol 31(3-4): 415-430.

Lennartz, M. R., J. B. Lefkowith, F. A. Bromley and E. J. Brown (1993). "Immunoglobulin G-mediated phagocytosis activates a calcium-independent, phosphatidylethanolamine-specific phospholipase." J Leukoc Biol 54(5): 389-398.

Lesprit, P., F. Faurisson, O. Join-Lambert, F. Roudot-Thoraval, M. Foglino, C. Vissuzaine and C. Carbon (2003). "Role of the quorum-sensing system in experimental pneumonia due to Pseudomonas aeruginosa in rats." Am J Respir Crit Care Med 167(11): 1478-1482.

Leverrier, Y., K. Okkenhaug, C. Sawyer, A. Bilancio, B. Vanhaesebroeck and A. J. Ridley (2003). "Class I phosphoinositide 3-kinase p110beta is required for apoptotic cell and Fcgamma receptor-mediated phagocytosis by macrophages." J Biol Chem 278(40): $38437-38442$.

Li, Q., C. Jagannath, P. K. Rao, C. R. Singh and G. Lostumbo (2010). "Analysis of phagosomal proteomes: from latex-bead to bacterial phagosomes." Proteomics 10(22): 4098-4116.

Li, Y., X. Meng, H. Feng, G. Zhang, C. Liu and P. Li (1999). "Over-expression of the RAB5 gene in human lung adenocarcinoma cells with high metastatic potential." Chin Med Sci J 14(2): 96-101.

Lorenz, E., D. C. Chemotti, K. Vandal and P. A. Tessier (2004). "Toll-like receptor 2 represses nonpilus adhesin-induced signaling in acute infections with the Pseudomonas aeruginosa pilA mutant." Infect Immun 72(8): 4561-4569.

Lowry, M. B., A. M. Duchemin, J. M. Robinson and C. L. Anderson (1998). "Functional separation of pseudopod extension and particle internalization during Fc gamma receptormediated phagocytosis." J Exp Med 187(2): 161-176.

Lundqvist-Gustafsson, H., M. Gustafsson and C. Dahlgren (2000). "Dynamic $\mathrm{ca}(2+)$ changes in neutrophil phagosomes A source for intracellular $\mathrm{ca}(2+)$ during phagolysosome formation?" Cell Calcium 27(6): 353-362. 
Lyczak, J. B., C. L. Cannon and G. B. Pier (2000). "Establishment of Pseudomonas aeruginosa infection: lessons from a versatile opportunist." Microbes Infect 2(9): 10511060 .

Madrazo, D. R., S. L. Tranguch and I. Marriott (2003). "Signaling via Toll-like receptor 5 can initiate inflammatory mediator production by murine osteoblasts." Infect Immun 71(9): 5418-5421.

Mahenthiralingam, E. and D. P. Speert (1995). "Nonopsonic phagocytosis of Pseudomonas aeruginosa by macrophages and polymorphonuclear leukocytes requires the presence of the bacterial flagellum." Infect Immun 63(11): 4519-4523.

Malik, Z. A., G. M. Denning and D. J. Kusner (2000). "Inhibition of Ca(2+) signaling by Mycobacterium tuberculosis is associated with reduced phagosome-lysosome fusion and increased survival within human macrophages." J Exp Med 191(2): 287-302.

Malik, Z. A., C. R. Thompson, S. Hashimi, B. Porter, S. S. Iyer and D. J. Kusner (2003). "Cutting edge: Mycobacterium tuberculosis blocks $\mathrm{Ca} 2+$ signaling and phagosome maturation in human macrophages via specific inhibition of sphingosine kinase." J Immunol 170(6): 2811-2815.

Maridonneau-Parini, I., C. Z. Yang, M. Bornens and B. Goud (1991). "Increase in the expression of a family of small guanosine triphosphate-binding proteins, rab proteins, during induced phagocyte differentiation." J Clin Invest 87(3): 901-907.

Mariencheck, W. I., J. F. Alcorn, S. M. Palmer and J. R. Wright (2003). "Pseudomonas aeruginosa elastase degrades surfactant proteins A and D." Am J Respir Cell Mol Biol 28(4): 528-537.

Marshall, J. G., J. W. Booth, V. Stambolic, T. Mak, T. Balla, A. D. Schreiber, T. Meyer and S. Grinstein (2001). "Restricted accumulation of phosphatidylinositol 3-kinase products in a plasmalemmal subdomain during Fc gamma receptor-mediated phagocytosis." J Cell Biol 153(7): 1369-1380.

Mavrodi, D. V., V. N. Ksenzenko, R. F. Bonsall, R. J. Cook, A. M. Boronin and L. S. Thomashow (1998). "A seven-gene locus for synthesis of phenazine-1-carboxylic acid by Pseudomonas fluorescens 2-79." J Bacteriol 180(9): 2541-2548.

McBride, H. M., V. Rybin, C. Murphy, A. Giner, R. Teasdale and M. Zerial (1999). "Oligomeric complexes link Rab5 effectors with NSF and drive membrane fusion via interactions between EEA1 and syntaxin 13." Cell 98(3): 377-386.

Medzhitov, R. (2007). "Recognition of microorganisms and activation of the immune response." Nature 449(7164): 819-826.

Mehta, N. G. (1976). "Recognition of self and nonself, the crucial role of phagocytosis and lysosomal destruction of antigen." Med Hypotheses 2(4): 141-146. 
Meyer, J. M., A. Neely, A. Stintzi, C. Georges and I. A. Holder (1996). "Pyoverdin is essential for virulence of Pseudomonas aeruginosa." Infect Immun 64(2): 518-523.

Milatovic, D. and I. Braveny (1987). "Development of resistance during antibiotic therapy." Eur J Clin Microbiol 6(3): 234-244.

Mustafi, S., Barbieri, M.A., Kim, L.W (2013). "Microorganisms: Modulation of Phagocytosis and its Mechanisms of Action." Current Cellular Biochemistry 3(1): 1.

Nielsen, E., S. Christoforidis, S. Uttenweiler-Joseph, M. Miaczynska, F. Dewitte, M. Wilm, B. Hoflack and M. Zerial (2000). "Rabenosyn-5, a novel Rab5 effector, is complexed with hVPS45 and recruited to endosomes through a FYVE finger domain." J Cell Biol 151(3): 601-612.

Nunes, P. and N. Demaurex (2010). "The role of calcium signaling in phagocytosis." J Leukoc Biol 88(1): 57-68.

O'Neill, L. A. (2002). "Toll-like receptor signal transduction and the tailoring of innate immunity: a role for Mal?" Trends Immunol 23(6): 296-300.

Oancea, E. and T. Meyer (1998). "Protein kinase C as a molecular machine for decoding calcium and diacylglycerol signals." Cell 95(3): 307-318.

Odin, J. A., J. C. Edberg, C. J. Painter, R. P. Kimberly and J. C. Unkeless (1991). "Regulation of phagocytosis and [Ca2+]i flux by distinct regions of an $\mathrm{Fc}$ receptor." Science 254(5039): 1785-1788.

Ofek, I., J. Goldhar, Y. Keisari and N. Sharon (1995). "Nonopsonic phagocytosis of microorganisms." Annu Rev Microbiol 49: 239-276.

Ojaniemi, M., V. Glumoff, K. Harju, M. Liljeroos, K. Vuori and M. Hallman (2003). "Phosphatidylinositol 3-kinase is involved in Toll-like receptor 4-mediated cytokine expression in mouse macrophages." Eur J Immunol 33(3): 597-605.

Passador, L., J. M. Cook, M. J. Gambello, L. Rust and B. H. Iglewski (1993). "Expression of Pseudomonas aeruginosa virulence genes requires cell-to-cell communication." Science 260(5111): 1127-1130.

Patel, J. C., A. Hall and E. Caron (2002). "Vav regulates activation of Rac but not Cdc42 during FcgammaR-mediated phagocytosis." Mol Biol Cell 13(4): 1215-1226.

Patel, P. C. and R. E. Harrison (2008). "Membrane ruffles capture C3bi-opsonized particles in activated macrophages." Mol Biol Cell 19(11): 4628-4639.

Pechere, J. C. and I. R. Vladoianu (1992). "Development of resistance during ceftazidime and cefepime therapy in a murine peritonitis model." J Antimicrob Chemother 29(5): 563-573. 
Peiser, L. and S. Gordon (2001). "The function of scavenger receptors expressed by macrophages and their role in the regulation of inflammation." Microbes Infect 3(2): 149159.

Pereira-Leal, J. B. and M. C. Seabra (2000). "The mammalian Rab family of small GTPases: definition of family and subfamily sequence motifs suggests a mechanism for functional specificity in the Ras superfamily." J Mol Biol 301(4): 1077-1087.

Pfeffer, S. R. (2001). "Rab GTPases: specifying and deciphering organelle identity and function." Trends Cell Biol 11(12): 487-491.

Pikkarainen, T., A. Brannstrom and K. Tryggvason (1999). "Expression of macrophage MARCO receptor induces formation of dendritic plasma membrane processes." J Biol Chem 274(16): 10975-10982.

Pohlmann, R., M. W. Boeker and K. von Figura (1995). "The two mannose 6-phosphate receptors transport distinct complements of lysosomal proteins." J Biol Chem 270(45): 27311-27318.

Power, M. R., Y. Peng, E. Maydanski, J. S. Marshall and T. J. Lin (2004). "The development of early host response to Pseudomonas aeruginosa lung infection is critically dependent on myeloid differentiation factor 88 in mice." J Biol Chem 279(47): $49315-49322$.

Quinn, J. P., E. J. Dudek, C. A. DiVincenzo, D. A. Lucks and S. A. Lerner (1986). "Emergence of resistance to imipenem during therapy for Pseudomonas aeruginosa infections." J Infect Dis 154(2): 289-294.

Ravetch, J. V. (1994). "Fc receptors: rubor redux." Cell 78(4): 553-560.

Ravetch, J. V. (1997). "Fc receptors." Curr Opin Immunol 9(1): 121-125.

Ravikumar, B., S. Imarisio, S. Sarkar, C. J. O'Kane and D. C. Rubinsztein (2008). "Rab5 modulates aggregation and toxicity of mutant huntingtin through macroautophagy in cell and fly models of Huntington disease." J Cell Sci 121(Pt 10): 1649-1660.

Reddien, P. W., S. Cameron and H. R. Horvitz (2001). "Phagocytosis promotes programmed cell death in C. elegans." Nature 412(6843): 198-202.

Reth, M. (1989). "Antigen receptor tail clue." Nature 338(6214): 383-384.

Roberts, E. A., J. Chua, G. B. Kyei and V. Deretic (2006). "Higher order Rab programming in phagolysosome biogenesis." J Cell Biol 174(7): 923-929.

Roberts, R. L., M. A. Barbieri, J. Ullrich and P. D. Stahl (2000). "Dynamics of rab5 activation in endocytosis and phagocytosis." J Leukoc Biol 68(5): 627-632. 
Roger, T., J. David, M. P. Glauser and T. Calandra (2001). "MIF regulates innate immune responses through modulation of Toll-like receptor 4." Nature 414(6866): 920924.

Romano, P. S., M. G. Gutierrez, W. Beron, M. Rabinovitch and M. I. Colombo (2007). "The autophagic pathway is actively modulated by phase II Coxiella burnetii to efficiently replicate in the host cell." Cell Microbiol 9(4): 891-909.

Rosenberger, C. M. and B. B. Finlay (2003). "Phagocyte sabotage: disruption of macrophage signalling by bacterial pathogens." Nat Rev Mol Cell Biol 4(5): 385-396.

Roy-Burman, A., R. H. Savel, S. Racine, B. L. Swanson, N. S. Revadigar, J. Fujimoto, T. Sawa, D. W. Frank and J. P. Wiener-Kronish (2001). "Type III protein secretion is associated with death in lower respiratory and systemic Pseudomonas aeruginosa infections." J Infect Dis 183(12): 1767-1774.

Rudolph, M. G., P. Bayer, A. Abo, J. Kuhlmann, I. R. Vetter and A. Wittinghofer (1998). "The Cdc42/Rac interactive binding region motif of the Wiskott Aldrich syndrome protein (WASP) is necessary but not sufficient for tight binding to Cdc42 and structure formation." J Biol Chem 273(29): 18067-18076.

Saito, K., J. Murai, H. Kajiho, K. Kontani, H. Kurosu and T. Katada (2002). "A novel binding protein composed of homophilic tetramer exhibits unique properties for the small GTPase Rab5." J Biol Chem 277(5): 3412-3418.

Santucci, S. G., S. Gobara, C. R. Santos, C. Fontana and A. S. Levin (2003). "Infections in a burn intensive care unit: experience of seven years." J Hosp Infect 53(1): 6-13.

Sastry, K. and R. A. Ezekowitz (1993). "Collectins: pattern recognition molecules involved in first line host defense." Curr Opin Immunol 5(1): 59-66.

Sato, H., D. W. Frank, C. J. Hillard, J. B. Feix, R. R. Pankhaniya, K. Moriyama, V. Finck-Barbancon, A. Buchaklian, M. Lei, R. M. Long, J. Wiener-Kronish and T. Sawa (2003). "The mechanism of action of the Pseudomonas aeruginosa-encoded type III cytotoxin, ExoU." EMBO J 22(12): 2959-2969.

Sawyer, D. W., J. A. Sullivan and G. L. Mandell (1985). "Intracellular free calcium localization in neutrophils during phagocytosis." Science 230(4726): 663-666.

Schiller, N. L. and K. A. Joiner (1986). "Interaction of complement with serum-sensitive and serum-resistant strains of Pseudomonas aeruginosa." Infect Immun 54(3): 689-694.

Schmidtchen, A., E. Holst, H. Tapper and L. Bjorck (2003). "Elastase-producing Pseudomonas aeruginosa degrade plasma proteins and extracellular products of human skin and fibroblasts, and inhibit fibroblast growth." Microb Pathog 34(1): 47-55. 
Schulert, G. S., H. Feltman, S. D. Rabin, C. G. Martin, S. E. Battle, J. Rello and A. R. Hauser (2003). "Secretion of the toxin ExoU is a marker for highly virulent Pseudomonas aeruginosa isolates obtained from patients with hospital-acquired pneumonia." J Infect Dis 188(11): 1695-1706.

Schultz, M. J., S. Knapp, S. Florquin, J. Pater, K. Takeda, S. Akira and T. van der Poll (2003). "Interleukin-18 impairs the pulmonary host response to Pseudomonas aeruginosa." Infect Immun 71(4): 1630-1634.

Scott, C. C., W. Furuya, W. S. Trimble and S. Grinstein (2003). "Activation of storeoperated calcium channels: assessment of the role of snare-mediated vesicular transport." J Biol Chem 278(33): 30534-30539.

Seabra, M. C., E. H. Mules and A. N. Hume (2002). "Rab GTPases, intracellular traffic and disease." Trends Mol Med 8(1): 23-30.

Shaver, C. M. and A. R. Hauser (2004). "Relative contributions of Pseudomonas aeruginosa ExoU, ExoS, and ExoT to virulence in the lung." Infect Immun 72(12): 69696977.

Shaw, D. R. and F. M. Griffin, Jr. (1981). "Phagocytosis requires repeated triggering of macrophage phagocytic receptors during particle ingestion." Nature 289(5796): 409-411.

Shields, J. M., K. Pruitt, A. McFall, A. Shaub and C. J. Der (2000). "Understanding Ras: 'it ain't over 'til it's over'." Trends Cell Biol 10(4): 147-154.

Silverstein, R. L., W. Li, Y. M. Park and S. O. Rahaman (2010). "Mechanisms of cell signaling by the scavenger receptor CD36: implications in atherosclerosis and thrombosis." Trans Am Clin Climatol Assoc 121: 206-220.

Simonsen, A., R. Lippe, S. Christoforidis, J. M. Gaullier, A. Brech, J. Callaghan, B. H. Toh, C. Murphy, M. Zerial and H. Stenmark (1998). "EEA1 links PI(3)K function to Rab5 regulation of endosome fusion." Nature 394(6692): 494-498.

Smith, R. S., E. R. Fedyk, T. A. Springer, N. Mukaida, B. H. Iglewski and R. P. Phipps (2001). "IL-8 production in human lung fibroblasts and epithelial cells activated by the Pseudomonas autoinducer N-3-oxododecanoyl homoserine lactone is transcriptionally regulated by NF-kappa B and activator protein-2." J Immunol 167(1): 366-374.

Smith, R. S., S. G. Harris, R. Phipps and B. Iglewski (2002). "The Pseudomonas aeruginosa quorum-sensing molecule $\mathrm{N}$-(3-oxododecanoyl)homoserine lactone contributes to virulence and induces inflammation in vivo." J Bacteriol 184(4): 11321139.

Smith, R. S. and B. H. Iglewski (2003). "Pseudomonas aeruginosa quorum sensing as a potential antimicrobial target." J Clin Invest 112(10): 1460-1465. 
Smith, R. S., R. Kelly, B. H. Iglewski and R. P. Phipps (2002). "The Pseudomonas autoinducer $\mathrm{N}$-(3-oxododecanoyl) homoserine lactone induces cyclooxygenase-2 and prostaglandin E2 production in human lung fibroblasts: implications for inflammation." J Immunol 169(5): 2636-2642.

Soong, G., B. Reddy, S. Sokol, R. Adamo and A. Prince (2004). "TLR2 is mobilized into an apical lipid raft receptor complex to signal infection in airway epithelial cells." J Clin Invest 113(10): 1482-1489.

Speert, D. P. and S. Gordon (1992). "Phagocytosis of unopsonized Pseudomonas aeruginosa by murine macrophages is a two-step process requiring glucose." J Clin Invest 90(3): 1085-1092.

Speert, D. P., S. D. Wright, S. C. Silverstein and B. Mah (1988). "Functional characterization of macrophage receptors for in vitro phagocytosis of unopsonized Pseudomonas aeruginosa." J Clin Invest 82(3): 872-879.

Stahl, P. D. and R. A. Ezekowitz (1998). "The mannose receptor is a pattern recognition receptor involved in host defense." Curr Opin Immunol 10(1): 50-55.

Stein, M. P., J. Dong and A. Wandinger-Ness (2003). "Rab proteins and endocytic trafficking: potential targets for therapeutic intervention." Adv Drug Deliv Rev 55(11): 1421-1437.

Stendahl, O. I., J. H. Hartwig, E. A. Brotschi and T. P. Stossel (1980). "Distribution of actin-binding protein and myosin in macrophages during spreading and phagocytosis." $\underline{J}$ Cell Biol 84(2): 215-224.

Sumi, T., K. Matsumoto, Y. Takai and T. Nakamura (1999). "Cofilin phosphorylation and actin cytoskeletal dynamics regulated by rho- and Cdc42-activated LIM-kinase 2." J Cell Biol 147(7): 1519-1532.

Sun, Y., M. Karmakar, P. R. Taylor, A. Rietsch and E. Pearlman (2012). "ExoS and ExoT ADP ribosyltransferase activities mediate Pseudomonas aeruginosa keratitis by promoting neutrophil apoptosis and bacterial survival." J Immunol 188(4): 1884-1895.

Suzuki, H., Y. Kurihara, M. Takeya, N. Kamada, M. Kataoka, K. Jishage, O. Ueda, H. Sakaguchi, T. Higashi, T. Suzuki, Y. Takashima, Y. Kawabe, O. Cynshi, Y. Wada, M. Honda, H. Kurihara, H. Aburatani, T. Doi, A. Matsumoto, S. Azuma, T. Noda, Y. Toyoda, H. Itakura, Y. Yazaki, T. Kodama and et al. (1997). "A role for macrophage scavenger receptors in atherosclerosis and susceptibility to infection." Nature 386(6622): 292-296.

Swanson, J. A. and A. D. Hoppe (2004). "The coordination of signaling during Fc receptor-mediated phagocytosis." J Leukoc Biol 76(6): 1093-1103. 
Swanson, J. A., M. T. Johnson, K. Beningo, P. Post, M. Mooseker and N. Araki (1999). "A contractile activity that closes phagosomes in macrophages." J Cell Sci 112 ( Pt 3): 307-316.

Tacconelli, E., M. Tumbarello, S. Bertagnolio, R. Citton, T. Spanu, G. Fadda and R. Cauda (2002). "Multidrug-resistant Pseudomonas aeruginosa bloodstream infections: analysis of trends in prevalence and epidemiology." Emerg Infect Dis 8(2): 220-221.

Takai, S., M. Kasama, K. Yamada, N. Kai, N. Hirayama, H. Namiki and T. Taniyama (1994). "Human high-affinity Fc gamma RI (CD64) gene mapped to chromosome 1q21.2-q21.3 by fluorescence in situ hybridization." Hum Genet 93(1): 13-15.

Takase, H., H. Nitanai, K. Hoshino and T. Otani (2000). "Impact of siderophore production on Pseudomonas aeruginosa infections in immunosuppressed mice." Infect Immun 68(4): 1834-1839.

Takeda, K., T. Kaisho and S. Akira (2003). "Toll-like receptors." Annu Rev Immunol 21: 335-376.

Takenawa, T. and H. Miki (2001). "WASP and WAVE family proteins: key molecules for rapid rearrangement of cortical actin filaments and cell movement." J Cell Sci 114(Pt 10): 1801-1809.

Tall, G. G., M. A. Barbieri, P. D. Stahl and B. F. Horazdovsky (2001). "Ras-activated endocytosis is mediated by the Rab5 guanine nucleotide exchange activity of RIN1." Dev Cell 1(1): 73-82.

Tang, H., M. Kays and A. Prince (1995). "Role of Pseudomonas aeruginosa pili in acute pulmonary infection." Infect Immun 63(4): 1278-1285.

Targowski, S. P. and W. Klucinski (1985). "Effect of immune complexes from mastitic milk on blocking of Fc receptors and phagocytosis." Infect Immun 47(2): 484-488.

Tauber, A. I. (2003). "Metchnikoff and the phagocytosis theory." Nat Rev Mol Cell Biol 4(11): 897-901.

Telford, G., D. Wheeler, P. Williams, P. T. Tomkins, P. Appleby, H. Sewell, G. S. Stewart, B. W. Bycroft and D. I. Pritchard (1998). "The Pseudomonas aeruginosa quorum-sensing signal molecule $\mathrm{N}$-(3-oxododecanoyl)-L-homoserine lactone has immunomodulatory activity." Infect Immun 66(1): 36-42.

Thomas, C. A., Y. Li, T. Kodama, H. Suzuki, S. C. Silverstein and J. El Khoury (2000). "Protection from lethal gram-positive infection by macrophage scavenger receptordependent phagocytosis." J Exp Med 191(1): 147-156. 
Tridandapani, S., T. W. Lyden, J. L. Smith, J. E. Carter, K. M. Coggeshall and C. L. Anderson (2000). "The adapter protein LAT enhances fcgamma receptor-mediated signal transduction in myeloid cells." J Biol Chem 275(27): 20480-20487.

Trinidad, A. G., M. L. de la Puerta, N. Fernandez, Y. Bayon, M. S. Crespo and A. Alonso (2006). "Coupling of C3bi to IgG inhibits the tyrosine phosphorylation signaling cascade downstream Syk and reduces cytokine induction in monocytes." J Leukoc Biol 79(5): 1073-1082.

Trouillet, J. L., A. Vuagnat, A. Combes, N. Kassis, J. Chastre and C. Gibert (2002). "Pseudomonas aeruginosa ventilator-associated pneumonia: comparison of episodes due to piperacillin-resistant versus piperacillin-susceptible organisms." Clin Infect Dis 34(8): 1047-1054.

Underhill, D. M. (2005). "Phagosome maturation: steady as she goes." Immunity 23(4): $343-344$.

Vance, R. E., A. Rietsch and J. J. Mekalanos (2005). "Role of the type III secreted exoenzymes S, T, and $\mathrm{Y}$ in systemic spread of Pseudomonas aeruginosa PAO1 in vivo." Infect Immun 73(3): 1706-1713.

Vasil, M. L. and U. A. Ochsner (1999). "The response of Pseudomonas aeruginosa to iron: genetics, biochemistry and virulence." Mol Microbiol 34(3): 399-413.

Venkateswarlu, K. and P. J. Cullen (2000). "Signalling via ADP-ribosylation factor 6 lies downstream of phosphatidylinositide 3-kinase." Biochem J 345 Pt 3: 719-724.

Vergne, I., R. A. Fratti, P. J. Hill, J. Chua, J. Belisle and V. Deretic (2004). "Mycobacterium tuberculosis phagosome maturation arrest: mycobacterial phosphatidylinositol analog phosphatidylinositol mannoside stimulates early endosomal fusion." Mol Biol Cell 15(2): 751-760.

Via, L. E., D. Deretic, R. J. Ulmer, N. S. Hibler, L. A. Huber and V. Deretic (1997). "Arrest of mycobacterial phagosome maturation is caused by a block in vesicle fusion between stages controlled by rab5 and rab7." J Biol Chem 272(20): 13326-13331.

Vieira, O. V., R. J. Botelho and S. Grinstein (2002). "Phagosome maturation: aging gracefully." Biochem J 366(Pt 3): 689-704.

Vieira, O. V., R. J. Botelho, L. Rameh, S. M. Brachmann, T. Matsuo, H. W. Davidson, A. Schreiber, J. M. Backer, L. C. Cantley and S. Grinstein (2001). "Distinct roles of class I and class III phosphatidylinositol 3-kinases in phagosome formation and maturation." J Cell Biol 155(1): 19-25.

Vieira, O. V., C. Bucci, R. E. Harrison, W. S. Trimble, L. Lanzetti, J. Gruenberg, A. D. Schreiber, P. D. Stahl and S. Grinstein (2003). "Modulation of Rab5 and Rab7 
recruitment to phagosomes by phosphatidylinositol 3-kinase." Mol Cell Biol 23(7): 25012514.

Vitale, G., V. Rybin, S. Christoforidis, P. Thornqvist, M. McCaffrey, H. Stenmark and M. Zerial (1998). "Distinct Rab-binding domains mediate the interaction of Rabaptin-5 with GTP-bound Rab4 and Rab5." EMBO J 17(7): 1941-1951.

Wilson, R., T. Pitt, G. Taylor, D. Watson, J. MacDermot, D. Sykes, D. Roberts and P. Cole (1987). "Pyocyanin and 1-hydroxyphenazine produced by Pseudomonas aeruginosa inhibit the beating of human respiratory cilia in vitro." J Clin Invest 79(1): 221-229.

Xiao, R. and W. S. Kisaalita (1997). "Iron acquisition from transferrin and lactoferrin by Pseudomonas aeruginosa pyoverdin." Microbiology 143 ( Pt 7): 2509-2515.

Yahr, T. L., A. J. Vallis, M. K. Hancock, J. T. Barbieri and D. W. Frank (1998). "ExoY, an adenylate cyclase secreted by the Pseudomonas aeruginosa type III system." Proc Natl Acad Sci U S A 95(23): 13899-13904.

Yates, R. M. and D. G. Russell (2005). "Phagosome maturation proceeds independently of stimulation of toll-like receptors 2 and 4." Immunity 23(4): 409-417.

Yates, S. P. and A. R. Merrill (2001). "A catalytic loop within Pseudomonas aeruginosa exotoxin A modulates its transferase activity." J Biol Chem 276(37): 35029-35036.

Younger, J. G., S. Shankar-Sinha, M. Mickiewicz, A. S. Brinkman, G. A. Valencia, J. V. Sarma, E. M. Younkin, T. J. Standiford, F. S. Zetoune and P. A. Ward (2003). "Murine complement interactions with Pseudomonas aeruginosa and their consequences during pneumonia." Am J Respir Cell Mol Biol 29(4): 432-438.

Zheleznyak, A. and E. J. Brown (1992). "Immunoglobulin-mediated phagocytosis by human monocytes requires protein kinase $\mathrm{C}$ activation. Evidence for protein kinase $\mathrm{C}$ translocation to phagosomes." J Biol Chem 267(17): 12042-12048. 


\section{Chapter 2}

\section{Regulations of Rab5 function in macrophages during phagocytosis of live}

\section{Pseudomonas aeruginosa.}

Mustafi, S., N. Rivero, J. C. Olson, P. D. Stahl and M. A. Barbieri (2013). "Regulation of

Rab5 function in macrophages during phagocytosis of live Pseudomonas aeruginosa." Infect Immun. 


\subsection{Abstract}

Pseudomonas aeruginosa, a Gram-negative opportunistic human pathogen, is a frequent cause of severe hospital-acquired infections. Effectors produced by the type III secretion system disrupt mammalian cell membrane trafficking and signaling and are integral to the establishment of $P$. aeruginosa infection. One of these effectors, ExoS, ADP-ribosylates several host cell proteins, including Ras and Rab GTPases. This current study demonstrated that Rab5 plays a critical role during early stages of $P$. aeruginosa invasion of J774-Eclone macrophages. Live, but not heat inactivated, P. aeruginosa inhibited phagocytosis, and this occurred in conjunction with down-regulation of Rab5 activity. Inactivation of Rab5 was dependent on ExoS ADP-ribosyltransferase activity, and in J744-Eclone cells, ExoS ADP-ribosyltransferase activity caused a more severe inhibition of phagocytosis than ExoS Rho GTPase activity. Furthermore, the expression of Rin1, a Rab5-guanine exchange factor, but not Rabex5 and Rap6, partially reversed the inactivation of Rab5 during invasion of live $P$. aeruginosa. These studies provide evidence that live $P$. aeruginosa are able to influence their rate of phagocytosis in macrophages by directly regulating activation of Rab5.

\subsection{Introduction}

Pseudomonas aeruginosa is a Gram-negative opportunistic pathogen capable of causing acute and chronic infections in immune-compromised individuals. Pseudomonas aeruginosa infection is also a serious problem for patients hospitalized with AIDS, cancer, cystic fibrosis, and burns (Pruitt 1974; Mendelson, Gurtman et al. 1994; Domingo, Ferre et al. 1998; Maschmeyer and Braveny 2000). The type III secretion (T3S) system allows Gram-negative bacteria to produce and translocate effector proteins 
into the cytoplasm of host cells. While the T3S system is conserved among distantly related pathogens, secreted effectors are pathogen specific (Hauser 2009). The secretion and translocation T3S effectors into the cytosol of animal or plant cells initiates a biochemical cross talk between pathogen and host (Hueck 1998). Four T3S effectors have been identified in P. aeruginosa: ExoS, ExoT, ExoU, and ExoY. Each effector functions differently to help create an environment inside the human host that favors bacterial survival and propagation in tissue.

The T3S effectors contribute to the ability of $P$. aeruginosa to invade tissue by breaking down physical barriers, damaging host cells, and conferring resistance to phagocytosis and host immune defenses (Lyczak, Cannon et al. 2000; Soong, Parker et al. 2008). Specifically, ExoS and ExoT are bi-functional effectors that have $76 \%$ homology and both include Rho GTPase activating (GAP) and ADP-ribosyltransferase (ADPr) activities (Barbieri and Sun 2004). The GAP activity of ExoS and ExoT functions similarly to inhibit $P$. aeruginosa internalization by inactivating Rho GTPases, Rho, Rac and Cdc42 that regulate actin cytoskeleton structure (Frithz-Lindsten, Du et al. 1997; Goehring, Schmidt et al. 1999; Cowell, Chen et al. 2000; Krall, Schmidt et al. 2000; Rocha, Coburn et al. 2003; Deng and Barbieri 2008). ExoS ADPr activity targets multiple specific substrates, including Ras-family proteins, such as Ras, RalA, Rac1 and Rabs to interrupt cell signaling (McGuffie, Frank et al. 1998; Fraylick, Riese et al. 2002; Henriksson, Sundin et al. 2002). The substrate specificity of ExoT ADPr activity differs from ExoS and is limited to $\mathrm{Crk}$ (CT10 regulator of kinase)-I, and Crk-II adaptor proteins that integrate protein tyrosine kinase signal transduction pathways (Liu, Yahr et al. 1997; Sun and Barbieri 2003; Deng, Sun et al. 2005). ExoU has been characterized as a 
necrotizing toxin with phospholipase activity (Sato, Frank et al. 2003) and has been found to block phagocyte-mediated clearance of infection (Diaz, Shaver et al. 2008). ExoY has adenylate cyclase activity and does not appear to play a major role in $P$. aeruginosa pathogenesis (Yahr, Vallis et al. 1998; Vance, Rietsch et al. 2005).

The Rab proteins, including Rab5, Rab7, Rab8 and Rab11, are known to be ADPribosylated by ExoS in vitro and in vivo (Fraylick, Rucks et al. 2002). The Rab proteins are a family of small GTP-binding proteins that regulate intracellular membrane trafficking of several pathogens, including Salmonella typhimurium (Mukherjee, Parashuraman et al. 2001; Madan, Krishnamurthy et al. 2008; Mallo, Espina et al. 2008), Mycobacterium spp. (Clemens, Lee et al. 2000), and Listeria monocytogenes (AlvarezDominguez, Barbieri et al. 1996). The Rab5 protein also functions in the phagocytosis of IgG opsonized particles (Duclos, Diez et al. 2000). In vitro studies have demonstrated that ExoS ADP-ribosylation of Rab5 diminishes the interaction between Rab5 and early endosome antigen 1 (EEA1) and fluid-phase uptake in intact cells Rab5 and its guanine exchange factors (GEFs), which include Rabex-5, Rin1 and Rap6 (also known as GAPex5) (Haas, Fuchs et al. 2005; Hunker, Galvis et al. 2006; Goh, Uchida et al. 2007; Olchowik and Miaczynska 2009), play a critical role in intracellular membrane trafficking (Stenmark, Parton et al. 1994), including phagocytosis of apoptotic cells (Nakaya, Tanaka et al. 2006). Although Rab5 was found to be present on phagosomes following phagocytosis of several bacterial pathogens and latex beads, the functional role for Rab5 in phagocytosis of $P$. aeruginosa is not fully understood.

This current study revealed that Rab5 activity was regulated during early stages of $P$. aeruginosa phagocytosis in J774-Eclone macrophages. Expression of wild-type Rab5 
(Rab5:WT) or a Rab5:Q79L, a GTP-hydrolysis defective mutant, increased invasion of heat-inactivated $P$. aeruginosa, while expression of Rab5:S34N, a GTP-binding defective mutant, decreased phagocytosis. Rab5 was activated during invasion of heat-inactivated P. aeruginosa, but inactivated during invasion of live $P$. aeruginosa. Expression of constitutively active Rab5:Q79L, overcame suppressive effects of live $P$. aeruginosa on phagocytosis. Inactivation of Rab5 by live P. aeruginosa was dependent on ExoS ADPr activity, and in J774-Eclone cells ExoS ADPr activity caused a more severe inhibition of phagocytosis than ExoS GAP activity. Finally, it was found that the expression of Rin1, a Rab5-GEF, interfered with the ability of live $P$. aeruginosa to inactivate Rab5. The ability of live $P$. aeruginosa to regulate phagocytosis by altering Rab5 activation provides further insight into how $P$. aeruginosa is able to manipulate the host during infection.

\subsection{Materials and Methods}

\subsubsection{Materials}

All chemicals and reagents were purchased from Sigma-Aldrich (Saint Louis, MO), unless otherwise indicated. Primary and secondary antibodies used in immunoblotting were purchased from Cell Signaling Technology Inc. (Danvers, MA). Culture supplies were purchased from Invitrogen life technologies (Carlsbad, CA).

\subsubsection{Cell culture}

The J774 E-clone cells (Diment, Leech et al. 1987) was kindly provided by Dr. Philip Stahl (Washington University Medical School, St Louis, MO) were maintained under a $5 \% \mathrm{CO}_{2}$ atmosphere in Dubelco's minimum essential medium (DMEM), supplemented with $10 \%$ heat-inactivated fetal calf serum (FCS), $2 \mathrm{mM}$ L-glutamine, 100 Units $/ \mathrm{mL}$ 
penicillin, $100 \mu \mathrm{g} / \mathrm{mL}$ of streptomycin. J774-Eclone cells were used for all P. aeruginosa phagocytosis studies. Platinum-E retroviral packaging cell line (Plat-E cells) were purchased from Cell Biolabs, Inc. (San Diego, CA) and maintained in DMEM, 10\% FCS, $1 \mu \mathrm{g} / \mathrm{mL}$ puromycin, $10 \mu \mathrm{g} / \mathrm{mL}$ blasticidin, 100 Units/ $\mathrm{mL}$ penicillin, $100 \mathrm{mg} / \mathrm{mL}$ of streptomycin.

\subsubsection{Bacteria strains}

Pseudomonas aeruginosa strains PAO1 (derivative of original Australian isolate PAO), PA103, which expresses ExoT and ExoU and naturally devoid of ExoS and

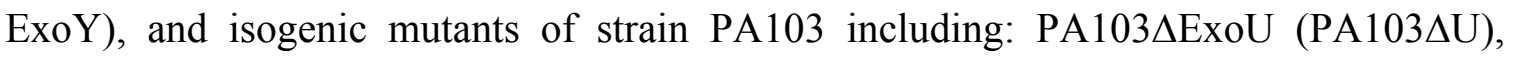
which expresses ExoT, and PA103 exoU exoT::Tc (PA103 $\Delta \mathrm{T} \Delta \mathrm{U})$ a T3S effector null mutant, were provided by Dara Frank (Medical College of Wisconsin, Milwaukee, WI). P. aeruginosa strains P103 $\Delta \mathrm{U} \Delta \mathrm{T}$ expressing:1) the pUCP vector (-), 2) wild-type ExoS (WT), 3) ExoS with active RhoGAP (RhoG+) but lacking ADPr activity due to E379A/E381A mutations, 4) ExoS with active ADPr activity (ADPr+) but lacking RhoGAP activity due to a R146A mutation, or 5) ExoS that lacks catalytic activity (RhoG-/ADPr-) due to E379A/E381A/R146A mutations, were previously described (Bridge, Martin et al. 2012). Bacteria were grown at $37^{\circ} \mathrm{C}$ in Lauria Broth with appropriate antibiotics. Prior to assay of phagocytosis, bacteria were grown to late-log phase and diluted to a concentration of $10^{7} \mathrm{cfu} / \mathrm{ml}$, and added to cells at indicated MOIs.

\subsubsection{Construction of recombinant pMX-puro retroviruses and cell lines}

The cDNAs of Rab4, Rab5, Rab7, Rin1, Rabex5 and Rap6 were subcloned into the pMX-puro vector as described (Barbieri, Fernandez-Pol et al. 2004). The cDNA of ExoS and ExoS deletion mutants containing RhoGAP (1 to 232 amino acids, ExoS:rRhoG) or 
ExoS ADPr (232 to 453 amino acids, ExoS:ADPr) domains were subcloned into the pMX-puro vector at BamH1 and Not1 sites, respectively. The cDNAs were used in the FUGENE6-mediated transfection of 90\% confluent Plate-E cell monolayers. Cells were maintained at $37^{\circ} \mathrm{C}$, and the medium containing released virus was harvested 48 hour after transfection. Viral stocks were aliquoted and frozen at $-80^{\circ} \mathrm{C}$ until use. Cell lines were generated by infecting J774-Eclone cells with retrovirus encoding GFP, Rab4, Rab5, Rab7, Rin1, Rabex5, Rap6 and ExoS domains, essentially as previously described (Barbieri, Fernandez-Pol et al. 2004).

\subsubsection{Phagocytosis assay}

Pseudomonas aeruginosa strains were cultured to late-log phase, washed with phosphate buffered saline (PBS, pH 7.3) and then with $\mathrm{NaHCO}_{3}, \mathrm{pH}$ 9, three times, respectively. After washing, Alexa fluor-594 (Invitrogen, Carlsbad, CA) was used to label live or heat-inactivated bacteria for 2 hours at room temperature while protected from light. J774-Eclone cells $\left(10^{5}\right.$ cells $\left./ \mathrm{ml}\right)$ were plated on coverslips in 6-well plates and incubated overnight. Cells were washed once with PBS and then twice with Hanks balanced salt solution-2\% BSA. Bacteria were added at a ratio of 200:1 and incubated for 30 minutes at $4{ }^{\circ} \mathrm{C}$. To initiate bacterial internalization, plates were placed in a $37^{\circ} \mathrm{C}$ water bath for 5 to 60 minutes. After this time, cells were placed on ice and washed three times with PBS, and then fixed for 20 minutes at room temperature using $3.7 \%$ paraformaldehyde. After fixation, cells were washed three times with PBS, incubated with $1 \%$ Triton $\mathrm{X}-100$ at room temperature for 15 minutes, and incubated with 4',6diamidino-2-phenylindole (Roche Applied Science, Indianapolis, IN) to stain the nucleus. Coverslips were removed from the wells, washed and mounted with Mowiol fluorescence 
mounting medium. The number of bacteria per cell was enumerated at $100 \mathrm{X}$ magnification using a phase-contrast inverted fluorescent microscope. Two hundred cells were counted per slide, and each experiment was repeated three times. The phagocytic index refers to the number of bacteria inside each cell. An antibiotic protection assay, described by Goldova et al. , (Angus, Evans et al. 2010) was used to analyze bacterial survival within macrophages. For this assay, following the indicated time of phagocytosis, cells were washed 3 times with PBS, and extracellular bacteria were killed by incubating cells with growth medium containing Amikacin $(400 \mu \mathrm{g} / \mathrm{ml})$. After washing, cells were incubated at $37^{\circ} \mathrm{C}$ for an additional 30 and 60 minutes to examine bacterial survival within macrophages. Finally, cells were washed with PBS, lysed with $0.5 \%$ TritonX-100, and lysates were plated on LB-agar, incubated overnight and bacterial colonies were enumerated.

\subsubsection{Isolation of Purified Phagosomes}

Phagosome containing live or dead P. aeruginosa was isolated as described by Mukherjee et al. , (Mukherjee, Parashuraman et al. 2001). Briefly, J774E-clone cells were seeded at $1 \times 10^{8}$ cells $/ \mathrm{ml}$, and live or heat-inactivated $P$. aeruginosa were added to cells at a concentration of $2 \times 10^{9}$ bacteria/ml, followed by synchronization of cells and bacteria at $4{ }^{\circ} \mathrm{C}$ for 1 hour in HBSS buffer (Mukherjee, Parashuraman et al. 2001). Cells were then treated with pre-warmed HBSS medium and incubated for 5 minutes at $37^{\circ} \mathrm{C}$. Bacterial uptake was stopped by the addition of ice-cold HBSS medium. Unbound bacteria were removed by washing cells three times, centrifuging at low speed $(300 \times \mathrm{g}$ for 5 minutes) between washes. Washed cells were resuspended at a concentration of $2 \mathrm{x}$ $10^{8}$ cells $/ \mathrm{ml}$ in homogenization buffer $(250 \mathrm{mM}$ sucrose, $0.5 \mathrm{mM}$ EGTA, $20 \mathrm{mM}$ 
HEPES-KOH, $\mathrm{pH}$ 7.2) and homogenized in a ball bearing homogenizer at $4^{\circ} \mathrm{C}$. Homogenates were centrifuged at a low speed ( $400 \mathrm{x} g$ for 5 minutes) at $4{ }^{\circ} \mathrm{C}$ to remove nuclei and unbroken cells. To obtain the phagosomal fraction, post-nuclear supernatants were diluted with homogenization buffer (1:3), followed by centrifugation at 12,000 $\mathrm{x} g$ for $15 \mathrm{~s}$ at $4^{\circ} \mathrm{C}$ (Mukherjee, Parashuraman et al. 2001). Phagosomal fractions were resuspended in $100 \mathrm{ml}$ of homogenization buffer containing protease inhibitors, loaded on a $1 \mathrm{ml} 12 \%$ sucrose cushion, centrifuged at $800 \times \mathrm{g}$ for 45 minutes at $4^{\circ} \mathrm{C}$, and purified phagosomes were recovered from the bottom of the tube. Bacterial viability in the phagosomes was determined following selective lysis of the phagosomal membrane with solubilization buffer (50 mM Tris- $\mathrm{HCl}, 150 \mathrm{mM} \mathrm{NaCl}$ and $0.5 \% \mathrm{NP} 40)$ and plating lysates on LB-agar plates. Bacterial colonies formed after overnight incubation were quantified as previously described (Goldova, Ulrych et al. 2011).

\subsubsection{Cell lysis and immunoblotting}

For immunoblot analysis, J774-Eclone cells were washed twice with PBS and then lysed with RIPA cell lysis buffer (50 mM Tris-HCl, $150 \mathrm{mM} \mathrm{NaCl}, 0.1 \%$ SDS, $0.5 \%$ sodium deoxycholate, $1 \%$ NP40) in the presence of protease and phosphatase inhibitors. Lysates were collected with cell scrapers and cleared by centrifugation. Prior to SDSPAGE, cell lysates were resuspended in SDS sample buffer $(60 \mathrm{mM}$ Tris- $\mathrm{HCl}, 1 \%(\mathrm{w} / \mathrm{v})$ SDS, $10 \%$ glycerol, $0.05 \%$ (w/v) bromophenol blue, $\mathrm{pH} 6.8$, with $2 \% \quad \beta$ mercaptoethanol). Samples were subjected to SDS-PAGE and transferred to nitrocellulose membranes for immunoblotting. Nitrocellulose membranes were incubated with blocking solution (TBS containing $0.1 \%$ Tween 20 and 5\% BSA) and were probed with the indicated antibodies. 


\subsubsection{RNAi constructs and transfection}

The RNAi directed against:

mouse Rin1 [5'-UUAUACAUUUGCUUCACACCUAAGC-3'], mouse Rabex5 [ 5'-UUUAUAGAGACGCGUCAUGAUGUGC-3'],

mouse Rap6 [5'-AAGAATCGATTACCTATAGCA-3'], mouse Rab5A [5'-AAGCACAGTCCTATGCAGATG-3'], mouse Rab5B [5'-AATCCGTGTGTTTAGATGACA-3'], or mouse Rab5C [5'-AAGCAGCCATTGTGGTCTATG-3'] were designed and synthesized by Ambion (Austin, TX). A scrambled RNAi was designed as a control [5'CACCUAAUCCGUGGUUCAA-3']. Prior to RNAi transfection, J774-Eclone cells were plated in growth medium without antibiotics at 30-50\% confluency. RNAi (20 nM final concentration) transfection was performed using Lipofectamine-2000 (Invitrogen, Carlsbad, CA), as specified by Invitrogen. After transfection, cells were used either for immunoblotting, phagocytosis or pull-down assays.

\subsubsection{In vitro pull down assays}

Cells were lysed using a buffer containing 20 mM HEPES (pH 7.5), $100 \mathrm{mM} \mathrm{NaCl}, 1$ mM DTT, $5 \mathrm{mM} \mathrm{MgCl}_{2}, 5 \%$ glycerol and 1\% Triton-X-100, supplemented with $1 \mathrm{mM}$ PMSF. Lysates were incubated with $100 \mathrm{ml}$ glutathione beads containing $10 \mu \mathrm{g}$ of GSTEEA1, followed by rocking for 1 hour at $4{ }^{\circ} \mathrm{C}$. After incubation, beads were washed three times with PBS. The pull-downs were subjected to SDS-PAGE and analyzed by immunoblotting using Rab5 antibodies. 


\subsubsection{Image quantification}

The NIH Image J64 was used to quantify Western blots after images were scanned at a grayscale amplification of 600 dpi. Digital images of the Western blot from cell lines were captured and loaded into Image J64, and-Rab5 specific bands, along with $\alpha$-tubulin bands, were assessed in each sample using the Analyze $\rightarrow$ Gels function, which allows for background correction. The ratio of Rab5 signal to $\alpha$-tubulin was calculated for each sample and served as an index of Rab5 expression. The index of expression for other proteins examined in this study was derived in a similar manner.

\subsubsection{Statistical analysis}

All samples in this study were analyzed in duplicate and each experiment was repeated three times. Values represent the mean \pm SEM of three independent experiments. To compare two groups, student's t test was utilized. $P<0.05$ was considered as statistically significant.

\subsection{Results}

\subsubsection{Rab5 is required for phagocytosis of heat-inactivated Pseudomonas aeruginosa by macrophages.}

Previous studies have found that Rab GTPases, including Rab5, are manipulated by bacteria during phagocytosis (Brumell and Scidmore 2007). To investigate the involvement of Rab GTPases in P. aeruginosa phagocytosis, the pMX-puro retroviral expression system was used to express Rab proteins in J774-Eclone macrophages. Initially, to examine if the retroviral expression system altered phagocytosis of $P$. aeruginosa, control (non-transfected) or GFP transfected J774-Eclone cells were incubated at $37^{\circ} \mathrm{C}$ with heat-inactivated strain PAO1 P. aeruginosa at a ratio of 200:1, 
and the phagocytic index was monitored (as described in Materials and Methods) over a 1 hour period. Figure 1A shows that the rate of phagocytosis of heat-inactivated $P$. aeruginosa was not altered in GFP-expressing cells, as compared with non-transfected control cells. Similarly, the phagocytic index of heat-inactivated $P$. aeruginosa was not altered relative to increasing ratio of bacteria to cells in GFP-expressing cells, as compared with non-transfected control cells (Figure 6A, inset). Therefore, results from these studies concluded that phagocytosis of heat-inactivated $P$. aeruginosa by J774Eclone macrophages was time and bacterial concentration dependent and not altered by the pMX-puro retroviral expression system $(P>0.05)$.

Next, the effect of increased expression of Rab5 proteins on phagocytosis of heatinactivated $P$. aeruginosa by J774-Eclone macrophages was examined. Cells expressing GFP alone, GFP-wild-type Rab5 (Rab5:WT), GFP-Rab5:Q79L (QL), a constitutively active GTP-hydrolysis defective mutant, or GFP-Rab5:S34N, a constitutively inactive GTP-binding defective mutant were incubated with heat-inactivated $P$. aeruginosa, and the phagocytic index was monitored over a 60 minutes period. Expression of Rab5:WT and the Rab5:Q79L mutant increased the rate of phagocytosis of heat-inactivated $P$. aeruginosa, in relation to internalization of GFP-expressing control cells at 60 minutes (Figure 6B). In comparison, expression of the Rab5:S34N mutant decreased $(P<0.05)$ the rate phagocytosis of heat-inactivated $P$. aeruginosa, as compared to GFP-expressing cells (Fig. 1B). Enhanced expression of the indicated Rab5 construct was confirmed by immunoblot analysis in lysates of J774-Eclone cells, using tubulin as a reference protein (Figure 6B, inset). 
To examine the role of other Rab GTPases in P. aeruginosa phagocytosis, cells expressing GFP alone, Rab7:WT, the Rab7:Q67L GTP-hydrolysis defective mutant, or the Rab7:S22N GTP-binding defective mutant, were incubated with heat-inactivated $P$. aeruginosa and monitored for phagocytosis over a 60 minutes period. The phagocytic index of J744-Eclone cells expressing Rab7:WT and Rab7:Q67L was greater than that of GFP-expressing cells after $30 \mathrm{~min}$, while phagocytosis in cells expressing Rab7:S22N was halted beyond 15 minutes (Figure 6C) $(P<0.05)$. Enhanced expression of the respective Rab7 construct in transfected J774-Eclone cells was confirmed by immunoblot analysis (Figure 6C, inset). Unlike Rab5 or Rab7, phagocytosis of heat-inactivated $P$. aeruginosa was not altered by transfection of Rab4 constructs. In analyses of Rab4, the relative phagocytic index of J774-Eclone cells after 30 minutes was: 1) GFP, $100 \pm 7 \%$; 2) Rab4:WT, $103 \pm 6 \%$; 3) Rab4:Q67L, $98 \pm 5 \%$; and 4) Rab4:S22N, $95 \pm 5 \%$.

Also, the effect of depletion of Rab5 proteins by RNA interference (RNAi) on the uptake of heat-inactivated $P$. aeruginosa was investigated. Based on immunoblot analysis, greater than $95 \%$ of Rab5 protein was depleted by RNAi duplexes that targeted all three Rab5 isoforms (Figure 6D, inset). Depletion of all, but not individual Rab5 isoforms (data not shown), resulted in $80 \pm 6 \%$ reduction of internalization of heat-inactivated $P$. aeruginosa, when compared with non-RNAi treated or scramble RNAi treated control cells (Figure 6D).

Collectively, these results show that Rab5 regulates early events in the phagocytosis of heat-inactivated P. aeruginosa, and that effects of Rab5 differ kinetically from those of Rab7 on $P$. aeruginosa internalization. In addition, the finding that constitutively active Rab5:Q79L increased the rate of phagocytosis while Rab5:S34N decreased the rate of 
phagocytosis provides evidence that Rab5 activation plays a role in the uptake of heatinactivated $P$. aeruginosa in J774-Eclone macrophages.

\subsubsection{Live Pseudomonas aeruginosa blocks Rab5 activation in macrophages.}

Evidence that Rab5 plays a role in phagocytosis of heat-inactivated $P$. aeruginosa led to investigate the role of Rab5 during phagocytosis of live P. aeruginosa. Unexpectedly, internalization of live (L) strain PAO1 into J774-Eclone macrophages was found to be 75 $\pm 4 \%$ lower than internalization of heat-inactivated or dead (D) P. aeruginosa after 5, 15 or 30 minutes of infection.

Further investigation revealed if enhanced expression of Rab5 proteins could overcome suppressive effects of live $P$. aeruginosa on phagocytosis. For these studies J774-Eclone cells expressing a GFP control or Rab5:WT, Rab5:Q79L, or Rab5:S34N were incubated with live or heat-inactivated $P$. aeruginosa and phagocytosis was analyzed after 15 minutes. A decrease in the internalization of live $P$. aeruginosa was observed in cells expressing GFP alone, Rab5:WT, or Rab5:S34N, when compared to internalization of heat-inactivated $P$. aeruginosa (Figure 7B). However, in the cells expressing Rab5:Q79L, internalization of live P. aeruginosa closely approximated that of the heat-inactivated $P$. aeruginosa. Phagocytosis of live $P$. aeruginosa was also inhibited by silencing all Rab5 isoforms (Figure 7C), but not by silencing individual Rab5 isoforms (data not shown). Collectively, these results demonstrate that Rab5 exerts different effects on the internalization of live or heat-inactivated $P$. aeruginosa, and that these differences are nullified by the expression of constitutively activated Rab5. The finding that interference of expression of all, but not individual Rab5 isoforms, inhibits 
phagocytosis of live $P$. aeruginosa also highlights the cooperative and redundant regulation of $P$. aeruginosa phagocytosis by the three Rab5 isoforms.

Since the cycling of Rab5 between active and inactive states is integral to $P$. aeruginosa internalization, the question remains whether Rab5 activation is regulated by live $P$. aeruginosa as a defense mechanism against phagocytosis. To explore the ability of live $P$. aeruginosa to regulate Rab5 function, the phagosomes carrying live or heatinactivated $P$. aeruginosa during early phagocytosis were first characterized. For these studies, cells were incubated with live or heat-inactivated strain PAO1 for 5 minutes at $37^{\circ} \mathrm{C}$, and phagosomes containing $P$. aeruginos $a$ were isolated and analyzed. Figure $8 \mathrm{~A}$ shows that phagososomes containing live or heat-inactivated $P$. aeruginosa both recruited Rab5 protein on the phagosomal membrane, but that Rab5 accumulation was significantly reduced in phagososomes containing live P. aeruginosa. Notably, Rab7 was not detected on phagosomes isolated after 5 minutes of internalization (Figure 8A). A representative image showing relative levels of Rab5 and Rab7, in phagosomes, in relation to tubulin and actin reference proteins, is shown in Figure 8A, inset.

The reduced accumulation of Rab5 on phagosomes containing live P. aeruginosa correlated with inhibition of Rab5 activation was also determined. In these studies, J774Eclone macrophages were incubated with live or heat-inactivated P. aeruginosa for 5 minutes at $37^{\circ} \mathrm{C}$, and active Rab5 in total cell lysates was assayed using a GST-EEA1 glutathione bead pull-down assay, detecting active GTP-Rab5 bound to EEA1 by immunoblot analysis using an anti-Rab5 antibody. As shown in Figure 8B, incubation with live but not with heat-inactivated $P$. aeruginosa, significantly inhibited Rab5 binding to GST-EEA1. A representative immunoblot (Figure 8B, inset) shows lack of 
binding of lysates from live, but not heat-inactivated P. aeruginosa treated cells to GSTEEA1, in relation to total Rab5 and GST proteins in lysates.

To examine the influence of live $P$. aeruginosis on activation of Rab5 during phagocytosis, J774-Eclone cells expressing Rab5:WT or the Rab5:Q79L constitutively mutant were incubated with live or heat-inactivated $P$. aeruginosa for 5 minutes at $37^{\circ} \mathrm{C}$, and total lysates were analyzed for active GTP-Rab5 using the GST-EEA1 pull-down assay. As shown in Figure 8C, incubation of cells with live P. aeruginosa, but not with heat-inactivated $P$. aeruginosa, inhibited Rab5 activation, and this inhibition was nullified by expression of constitutively active Rab5:Q79L. These results are shown in the representative immunoblot (Figure $8 \mathrm{C}$, inset). Together, these studies demonstrate that live $P$. aeruginosa regulate both recruitment and activation of Rab5 to early phagosomes.

\subsubsection{Exotoxin $S$ plays a critical role in Rab5 activation during phagocytosis of Pseudomonas aeruginosa.}

Results show that phagocytosis of live $P$. aeruginosa by macrophage down-regulates Rab5 activation. Previous studies found that ExoS can ADP-ribosylate Rab5 (Fraylick, Rucks et al. 2002; Henriksson, Sundin et al. 2002) and in vitro studies confirmed that ADP-ribosylation of Rab5 by ExoS interfered with its interaction with EEA1 (Barbieri, Sha et al. 2001). These findings imply the ability of ExoS to interfere with endosomal tethering during $P$. aeruginosa phagocytosis. To determine the relationship between ExoS expression and Rab5 activation during phagocytosis, $P$. aeruginosa strains lacking ExoS were compared with strain PAO1, which expresses ExoS, ExoT and ExoY, for their ability to interfere with Rab5 activation during phagocytosis. Pseudomonas aeruginosa 
strains that were examined include: 1) PA103, lacks ExoS but expresses ExoT and ExoU; 2) PA103 $\Delta U$, lacks ExoS, ExoY and ExoU, and 3) PA103 $\Delta \mathrm{T} \Delta \mathrm{U}$, lacks all known T3S effectors.

When internalization of live or heat inactivated $P$. aeruginosa was examined following incubation of J774-Eclone cells with these strains for 15 minutes, significant differences were observed in the internalization of live strains, whereas the phagocytic response was uniform for all the heat-inactivated strains (Figure 9A). Live PAO1, which produces ExoS, caused the greatest inhibition of phagocytic uptake $(75 \pm 4 \%)$ relative to heat inactivated strain PAO1. In comparison, live strain PA103, expressing ExoT and ExoU, inhibited phagocytosis by $40 \pm 6 \%$, which closely approximated the $34 \pm 6 \%$ inhibition of phagocytosis caused by live strain PA103 $\Delta \mathrm{U}$ that expresses only ExoT. Live strain PA103 $\Delta \mathrm{T} \Delta \mathrm{U}$, lacking all four T3S effectors, caused a $15 \pm 3 \%$ inhibition of phagocytosis. These results provide evidence that ExoS and ExoT, produced by strain PAO1, have a more pronounced role than ExoT alone, and produced by strain PA103, in inhibiting phagocytosis in J774-Eclone macrophages.

When the fate of bacteria internalized within J774-Eclone macrophages was examined, strain PA103 was found to be more sensitive to macrophage-mediated killing than strain PAO1. A $53 \pm 6 \%$ and $17 \pm 3 \%$ survival rate was detected for internalized strain PA103, after 30 and 60 minutes, respectively, which compared with a $75 \pm 5 \%$ and $58 \pm 5 \%$ survival rate for internalized strain PAO1, after 30 minutes and 60 minutes, relative to the total internalized bacteria after 15 minutes of phagocytosis (control 100 $\pm 5 \%$ )

Another approach revealed how the uptake and survival of PA103 and PAO1 strains within macrophages are related to the ability of these strains to alter Rab5 activity. For 
these studies, cells were incubated with live or dead P. aeruginosa strains, and cell lysates were examined for active GTP-bound Rab5 in the GST-EEA1 pull-down assay, described earlier. As shown in Figure 4B, incubation of cells with strain PAO1, but not with strain PA103, inhibited Rab5 activation, and as expected, internalization of heatinactivated P. aeruginosa did not alter Rab5 activation. Interestingly, strains PA103 and PA103 $\Delta U$ that express ExoT did not inhibit Rab5 activation (Figure 9B), even though these strains inhibited $P$. aeruginosa internalization (Figure 9A). A representative immunoblot (Figure 9B, inset) shows lack of binding of J774-Eclone lysates obtained following exposure to live PAO1 to GST-EEA1, while all other lysates bound GSTEEA1. These results indicate that inhibition of J774-Eclone phagocytosis by ExoS producing strain PAO1 occurs in conjunction with inhibition of Rab5 activation, while inhibition of phagocytosis by ExoT producing PA103 stains occurs independently of alterations in Rab5 activation.

Several approaches were then used to further investigate the role of ExoS and Rab5 activation in the invasion of live $P$. aeruginosa in J774-Eclone cells. First, to assess the role of ExoS in P. aeruginosa internalization, PA103 $\Delta \mathrm{U} \Delta \mathrm{T}$ strains lacking ExoS(-), expressing ExoS (WT) or expressing ExoS with mutations that inactivate its GAP $(\mathrm{ADPr}+)$, its $\mathrm{ADPr}(\mathrm{RhoG}+)$, or its $\mathrm{GAP}$ and $\mathrm{ADPr}$ activities (ADPr-/RhoG-) were subjected to phagocytosis by J774-Eclone macrophage. For these studies, cells were incubated with each of these live $P$. aeruginosa strains for 5 minutes, prior to washing and determination of the phagocytic index. As shown in Figure 10A, greater than $80 \%$ inhibition was observed in the uptake of live PA103 $\Delta \mathrm{U} \Delta \mathrm{T}$ expressing either ExoS (WT) or the $\operatorname{ExoS}(\mathrm{ADPr}+)$ mutant as compared to PA103 $\Delta \mathrm{U} \Delta \mathrm{T}$ either lacking ExoS (-) or 
expressing an ExoS (ADPr-/RhoG-) mutant. A significant but less pronounced inhibition of phagocytosis ( $\sim 40 \%$ ) was caused by live PA103 $\Delta \mathrm{U} \Delta \mathrm{T}$ expressing an ExoS (RhoG+) mutant. These results provide evidence that ExoS (ADPr) activity plays a more pronounced role in the anti-phagocytic activity of live PA103 $\Delta \mathrm{U} \Delta \mathrm{T}$ expressing ExoS than ExoS (RhoG) activity.

The anti-phagocytic effects of these strains were also investigated in relation to the formation of GTP-bound Rab5 during the invasion of live P. aeruginosa. A significant decrease ( $>90 \%$ inhibition) in the formation of the GTP-bound form of Rab5 was caused by live PA103 $\Delta \mathrm{U} \Delta \mathrm{T}$ expressing ExoS (WT) or an ExoS (ADPr+) mutant, as compared to PA103 $\Delta \mathrm{U} \Delta \mathrm{T}$ expressing an ExoS (ADPr-/RhoG-) mutant (Fig. 5B). Notably, PA103 $\Delta \mathrm{U} \Delta \mathrm{T}$ expressing an ExoS (RhoG+ mutant) did not inhibit formation of the GTP-bound form of Rab5 (Figure 10B), indicating that the ADPr domain of ExoS is essential to inactivation of Rab5 during phagocytosis of live P. aeruginosa.

The role of ExoS and ExoS domains in Rab5 activation upon co-expression of both Rab5 and ExoS constructs in J774-Eclone macrophages were further examined. As shown in Figure 10C, when GTP-bound Rab5 cells was assayed in J774-Eclone cells expressing Rab5:WT or the Rab5:Q79L mutant and transiently co-transfected with ExoS, cells expressing both Rab5:WT and ExoS failed to produce GTP-bound Rab5, but activated GTP-bound Rab5 was detected in cells co-expressing constitutively active Rab5:Q79L and ExoS. When the effects of the individual ExoS domains on activation of Rab5 were examined, Rab5 activation was inhibited in cells expressing Rab5:WT and the ExoS-ADPr (rADPr) domain, while co-expression Rab5:WT with ExoS-RhoGAP (rRhoG) did not interfere with Rab5 activation (Figure 10D). Collectively, these results 
are consistent ExoS ADP-ribosylation of Rab5 during infection with live P. aeruginosa leading to inactivation of Rab5 and interference of phagocytosis of $P$. aeruginosa.

\subsubsection{Selective role of Rab5-GEFs during internalization of Pseudomonas aeruginosa.}

The switching of Rab5 between its GTP-active and GDP-inactive form is regulated by Rab5-GEFs including, Rin1, Rabex5 and Rap6. Since P. aeruginosa expressing ExoS ADPr activity was found to inhibit Rab5 activation in J774-Eclone macrophage, hence it was hypothesized that the expression of Rab5-GEFs may overcome the inhibitory effect of live $P$. aeruginosa on Rab5 activation and $P$. aeruginosa invasion.

To test this hypothesis, cells expressing GFP, Rin1, Rabex5, or Rap6 were incubated in the presence of live or heat-inactivated strain PAO1, and P. aeruginosa internalization was assayed after 15 minutes. As shown in Figure 11A, internalization of live and heatinactivated $P$. aeruginosa was increased in Rin1 over-expressing cells, as compared to GFP-control cells. Over-expression of Rap6 also increased the internalization of live and heat-inactivated, but to a lesser extent than that of Rin1. In contrast, over-expression of Rabex5 did not significantly alter internalization of live or dead P. aeruginosa. Phagocytosis of live or dead P. aeruginosa did not alter the expression of Rab5-GEFs constructs (Figure 11B).

To further examine the role of Rab5-GEFs in P. aeruginosa internalization, the expression of Rab5-GEFs was suppressed by using RNAis specific for Rin1, Rabex5 or Rap6. Interference of Rin1 expression caused a $>60 \%$ inhibition of internalization of live or heat-inactivated P. aeruginosa, as compared to GFP-control cells (Figure 11C and D). In comparison, interference of Rabex 5 and Rap6 expression caused only a small decrease 
in internalization of live or heat- inactivated $P$. aeruginosa, as compared to GFP-control cells. Interference of expression of all three Rab5-GEFs, using a triple knockdown, caused $>80 \%$ inhibition of internalization of live or heat-inactivated $P$. aeruginosa. Treating cells with each RNAi depleted more than $95 \%$ of the targeted protein (Figure 11E-G). These results implicate the ability of Rin1 to enhance invasion of live or heatinactivated $P$. aeruginosa in J774-Eclone macrophages.

\subsubsection{Rin1 expression partially reverses the negative effect of Pseudomonas aeruginosa on Rab5 activation in macrophages.}

Establishment of the a role of Rin1 in the internalization of live or dead P. aeruginosa led to the hypothesis that activation of Rab5 by Rin1 may, at least in part, be responsible for enhancing the internalization of live $P$. aeruginosa. To determine whether Rin1 activity increases internalization of live $P$. aeruginosa, J774-Eclone cells expressing GFP, Rin1, Rabex5, or Rap6 were incubated in the presence of live or heat-inactivated strain PAO1 P. aeruginosa for 5 minutes, and then examined the formation of the GTPbound form of Rab5 using a GST-EEA1 pull-down assay. Figure 12, shows that Rin1 expression increased the amount of active GTP-bound Rab5 formed during the internalization of live or heat-inactivated $P$. aeruginosa. This is consistent with the observation that the expression of Rin1 increased the internalization of live or heatinactivated $P$. aeruginosa (Figure 10A).

Interestingly, when Rabex5 or Rap6 expressing J774-Eclone cells were incubated with live P. aeruginosa, a small amount of GTP-bound Rab5 in the GST-EEA1 pulldown assay was observed for only Rap6 expressing E-clone cells, but the increase in the Rab5 GTP-bound form was lower than that observed in Rin1-expressing cells (Figure 
12). Taken together, these results indicate that Rin1 plays a selective and critical role in the activation of Rab5 during live $P$. aeruginosa invasion, which is consistent with the observation that live $P$. aeruginosa inactivates Rab5, and this inhibitory effect can be partially reversed by the expression of Rin1.

\subsection{Discussion}

Type III secretion system of $P$. aeruginosa is known to modulate host cell endocytosis (Barbieri, Sha et al. 2001; Deng and Barbieri 2008). This study demonstrated that Rab5 plays a critical role during early steps in the phagocytosis of $P$. aeruginosa in J774-Eclone macrophages. It was found that invasion of live, but not heat-inactivated $P$. aeruginosa, down-regulates Rab5 activation, and that inactivation of Rab5 during invasion requires expression of ExoS. In support of ExoS being a key effector in Rab5 activation, it was confirmed that ExoS ADPr activity, but not ExoS RhoGAP, inhibits Rab5 activation. In addition, it was also found that the over-expression of Rin1 partially reverses inactivation of Rab5 by ExoS ADPr activity during invasion of live $P$. aeruginosa. These observations led to develop a model that portrays ExoS ADPr activity and its interference of Rab5 activation as integral to the diminished internalization of live, as compared to heat-inactivated P. aeruginosa, in J774-Eclone macrophages.

While live $P$. aeruginosa were found to inactive Rab5 during $P$. aeruginosa phagocytosis, Rab5 also influenced phagocytosis of heat-inactivated P. aeruginosa. Both Rab5:WT and constitutively active Rab5:Q79L up-regulated the phagocytic index of heat-inactivate $P$. aeruginosa by 2 to 4 fold, whereas expression of constitutively inactive Rab5:S34N reduced the phagocytic index by half, as compared to control cells. Unlike Rab5, Rab4 and Rab7 did not alter P. aeruginosa internalization during early stages, up 
to $15 \mathrm{~min}$, of phagocytosis, but the Rab7:S22N mutant was able to diminish $P$. aeruginosa internalization after 30 minutes. These observations indicate that Rab5 plays a key role during early, while Rab7 plays a role in later stages of phagocytosis of heat killed $P$. aeruginosa,

The Rab5 protein is a substrate of ExoS ADPr activity and has been found to interfere with Rab5 function in vitro (Barbieri, Sha et al. 2001). Pseudomonas aeruginosa strains lacking one or more of the four T3S effectors were used to examine the role of ExoS in phagocytosis in J774-Eclone macrophages. When the phagocytic index of strains PA103, PA103 $\Delta U$, PA103 $\Delta \mathrm{T} \Delta \mathrm{U}$ or PAO1 strains was determined, it was found that live strain PA103, lacking both ExoS and ExoY (Fleiszig, Wiener-Kronish et al. 1997), was engulfed $\sim 3$ fold higher than live strain PAO1, which expresses ExoS, ExoT and ExoY. Studies performed in parallel found PAO1 survival within macrophages to be enhanced (58 $\pm 5 \%$ survival) in comparison to strain PA103 (17 $\pm 3 \%$ survival), indicating increased susceptibility of strain PA103 to macrophage-mediated killing. Notably, the uptake of live PA103 was about $40 \pm 6 \%$ less than that of heat-inactivated PA103, which is attributed to ExoT RhoGAP activity of strain PA103 (Garrity-Ryan, Shafikhani et al. 2004). In this regard, uptake of strain PA103 $\Delta$ U lacking ExoS, ExoU and ExoY, but expressing ExoT, was $34 \pm 6 \%$, similar to that of strain PA103. Observations also revealed that the phagocytic index of strain PA103 $\Delta \mathrm{T} \Delta \mathrm{U}$, which lacks known T3S effectors, was significantly lower than that of heat-inactivated PA103 (Fig. 4A). Consistent with factors other than T3S effectors playing a role in phagocytosis of live $P$. aeruginosa, exotoxin $\mathrm{A}$, phospholipase $\mathrm{C}$, alkaline protease, elastase and cell surface lipopolysaccharides have all been shown to alter macrophage responses and hence impair 
bacterium internalization (Pavlovskis, Pollack et al. 1977; Ruhen, Holt et al. 1980; Wretlind and Pavlovskis 1983).

While investigating the effect of $P$. aeruginosa on the activity of Rab5 proteins, it was demonstrated that live $P$. aeruginosa, but not the heat-inactivated $P$. aeruginosa, is responsible for diminishing levels of active GTP-bound Rab5. The inhibitory effect of live $P$. aeruginosa on Rab5 activation was overcome by expression of the constitutively active Rab5:Q79L. Interestingly, it was observed that live PAO1, but not live PA103, PA103 $\Delta U$ or PA103 $\Delta T \Delta U$ inhibited Rab5 activation (Figure 9B). Since, strain PA103 lacks ExoS and ExoY, and because ExoY seems unlikely to affect Rab5 activation (Hauser, Fleiszig et al. 1998), it could be speculated that ExoS plays a role in the modulation of Rab5 activation during the internalization of live $P$. aeruginosa in J774Eclone macrophages. Consistent with this idea, it was previously shown that ExoS ADPr activity blocked both HRP uptake and EGFR trafficking to lysosomes in CHO and Hela cells, respectively (Barbieri, Sha et al. 2001; Deng and Barbieri 2008) In directly testing the role of ExoS in modulating Rab5 activation in J774-Eclone macrophages, it was found that strain PA103 $\Delta \mathrm{T} \Delta \mathrm{U}$ expressing ExoS (WT) or ExoS (ADPr) activity, but not ExoS (Rho-GAP) activity, diminished levels of GTP-bound Rab5, in conjunction with inhibition of $P$. aeruginosa internalization (Figure 10A and B). Similarly, transient expression of ExoS (WT) and ExoS (rADPr), but not ExoS (rRhoG), within J774-Eclone cells was found to diminish levels of active GTP-bound Rab5 (Figure 10D). Observations from this study showed that ExoS anti-internalization activity was mostly dependent on its ADPr activity which differs from previous studies in HeLa cells where the ExoS antiinternalization function was attributed to RhoGAP activity (Krall, Sun et al. 2002; Deng 
and Barbieri 2008). One explanation for this discrepancy could be due to the use of a different cell line, and cell line properties are known to influence the substrate specificity of ExoS (Rucks, Fraylick et al. 2003). The ability of ExoS ADPr, but not its RhoGAP activity, to inactivate Rab5 (Figure 10A) provides evidence for cell-type dependent mechanisms of phagocytosis, which can be differentiated by ExoS.

Previous studies found that Rab5 might undergo ADP-ribosylation on multiple arginine residues by ExoS (Barbieri, Sha et al. 2001).This observation, together with the fact that Rab5 is inactivated by ExoS:ADPr activity, and that ADP-ribosylation interferes with Rab5 interaction with EEA1, indicate that key functional residues within the GTP binding motif of Rab5 may be targeted by ExoS. Interestingly, $\mathrm{Arg}^{81}$ is located in switch II of Rab5 proteins, immediately downstream of the second GTP/GDP binding motif (Valencia, Chardin et al. 1991), and mutation of $\mathrm{Arg}^{81}$ partially affects Rab5 function (Li and Stahl 1993). Confirmation of $\mathrm{Arg}^{81}$ as well as other Arg residues in Rab5 as targets of ExoS ADPr activity is integral to understanding how ExoS affects Rab5 function.

Since the nucleotide status of Rab5 is integral for P. aeruginosa invasion, the role of Rab5-GEFs, Rabex5, Rap6 and Rin1 was investigated in regards to Rab5 activation during $P$. aeruginosa internalization. Over-expression of Rin1 enhanced the internalization of heat-inactivated P. aeruginosa as well as Rap6 to some extend (Figure 11A). Consistent with the importance of Rab5 activation to phagocytosis of heatinactivated $P$. aeruginosa, Rab5 was activated when each of the Rab5-GEFs was overexpressed, albeit to different degrees (Figure 12). However, when the nucleotide status of Rab5 was analyzed in the presence of live $P$. aeruginosa, only Rin1 significantly overcame the inactivation of Rab5 in macrophages (Figure 12). This finding is in 
agreement with the significant increase in the internalization of live $P$. aeruginosa in cells over-expressing Rin1 proteins. It is worth noting that Rap6 and Rabex5 also increased levels of active GTP-Rab5, but it was significantly less than expression of Rin1 (Figure 12). The involvement of Rin1 in Rab5 activation was corroborated by depletion of Rin1, which significantly inhibited the uptake of live P. aeruginosa (Figure 11C). These results demonstrated for the first time that Rin1 is an essential regulator of Rab5 activation during phagocytosis of live $P$. aeruginosa.

In conclusion, this chapter demonstrated that live $P$. aeruginosa, but not heatinactivated $P$. aeruginosa, down-regulates Rab5 function in conjunction with inhibition of phagocytosis in J774-Eclone macrophages. Reduced phagocytosis of live $P$. aeruginosa by macrophages was overcome by expressing a constitutively active Rab5:Q79L mutant. ExoS ADPr activity mediated P. aeruginosa inactivation of Rab5, and unlike previous studies, ExoS ADPr, rather than ExoS GAP activity, was a dominant inhibitor of $P$. aeruginosa internalization, highlighting cell line differences in mechanisms of $P$. aeruginosa internalization. This studies support the hypothesis that increased Rab5 activity can accelerate phagocytosis of live $P$. aeruginosa and increase its degradation in macrophages. The exact mechanism of action of ExoS toward Rab5 function in macrophages is currently under investigation. 


\subsection{FIGURE LEGENDS}
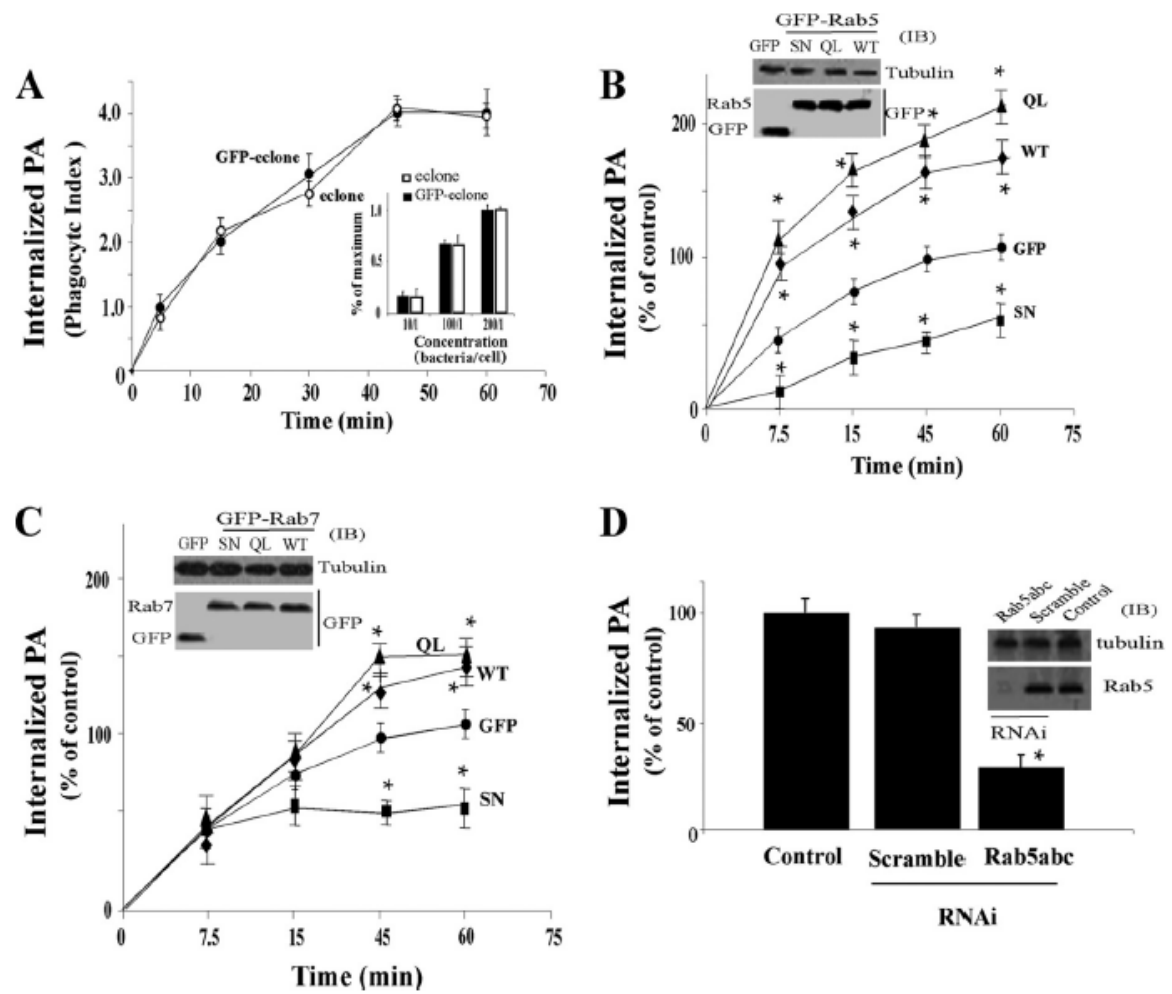

Figure 6: Effect of Rab proteins on invasion of heat-inactivated $P$. aeruginosa in macrophages.

(A) J774-Eclone cells alone or Eclone cells expressing GFP were incubated in the presence of heat-inactivated strain PAO1 P. aeruginosa at a ratio of 200:1 (bacteria/cell). At indicated times during a 60 minutes incubation period, cells were washed, fixed and the phagocytic index of $P$. aeruginosa (PA) was determined as described in Material and Methods. Inset: Eclone or GFP-Eclone cells were incubated with the indicated ratio of heat-inactivated $P$. aeruginosa for $60 \mathrm{~min}$, and the phagocytic index was determined and is expressed as percent of maximum, relative to the phagocytic index at the highest ratio of bacteria/cell (200/1). (B) J774-Eclone cells expressing GFP or Rab5:wild-type (WT), Rab5:Q79L (QL) or Rab5:S34N (SN) were incubated with heat-inactivated $P$. aeruginosa at a ratio of 200:1, and the phagocytic index was determined at the indicated times and is expressed as percent internalization relative to the GFP control at $60 \mathrm{~min}$. Inset: Cells expressing the indicated constructs were lysed and subjected to immunoblotting (IB) with anti-Rab5, anti-GFP or anti-tubulin antibodies. (C) J774Eclone cells expressing GFP or Rab7:WT, Rab7:Q67L (QL) or Rab7:S22N (SN) were incubated with heat-inactivated $P$. aeruginosa at a ratio of 200:1, and the phagocytic index was determined at the indicated times and is expressed as the percent of the GFP control at $60 \mathrm{~min}$. Inset: Cells expressing the indicated constructs were lysed and subjected to immunoblotting with anti-Rab7, anti-GFP or anti-tubulin antibodies. Results are representative of three independent experiments. (D) Non-transfected J774-Eclone 
cells (control), or Eclone cells transfected with scramble RNAi or RNAi's against Rab5 isoforms (Rab5 a,b,c) were incubated with heat-inactivated $P$. aeruginosa at a ratio of 200:1 for 60 minutes, and the phagocytic index was determined and expressed as percent of control cells. Inset: Cells transfected with the indicated RNAi's were lysed and subjected to immunoblotting with anti-tubulin or anti-Rab5 antibodies. Results represent the mean \pm SEM of three independent experiments. (*) represents statistically significant difference from control $(P<0.05)$. 

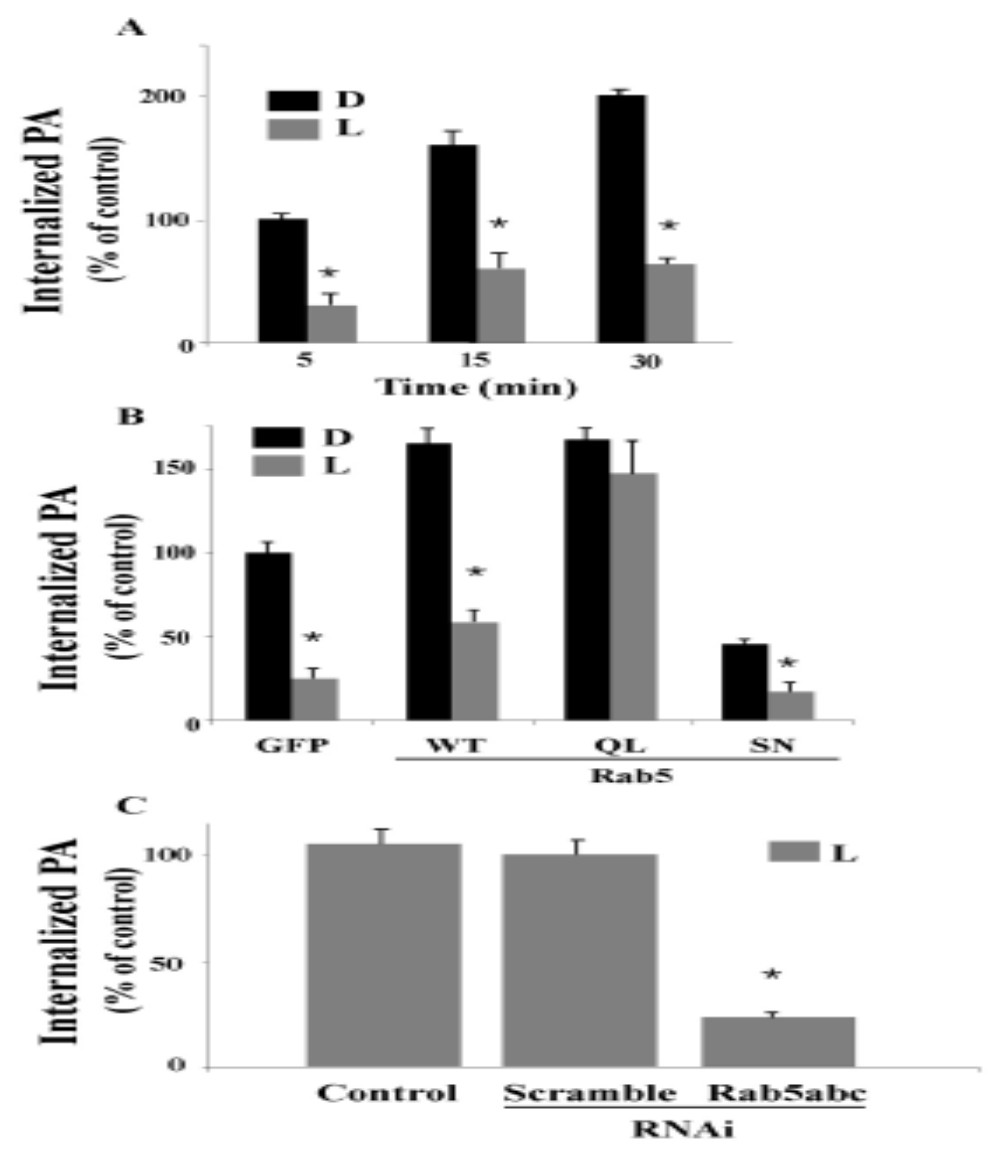

Figure 7: Effect of Rab5 on live $P$. aeruginosa invasion in macrophages.

(A) J774-Eclone cells expressing GFP were incubated with live (L) or heat-inactivated, dead (D) strain PAO1 P. aeruginosa at a ratio of 200:1 for 5, 15 or $30 \mathrm{~min}$. The phagocytic index was determined and is expressed as percent phagocytosis of heatinactivated bacteria internalized in GFP control cells at $5 \mathrm{~min}$. (B) J774-Eclone cells expressing GFP or Rab5:WT, Rab5:Q79L(QL) or Rab5:S34N (SN) were incubated with live or heat-inactivated $P$. aeruginosa at a ratio of $200: 1$ for 15 minutes at $37^{\circ} \mathrm{C}$. After incubation, the phagocytic index was determined and is expressed as percent phagocytosis of heat-inactivated bacteria by GFP control cells at $15 \mathrm{~min}$. (C) J774-Eclone cells alone (control) or cells transfected with scramble RNAi or RNAi's against Rab5 isoforms were incubated with heat-inactivated $P$. aeruginosa at a ratio of 200:1 for 30 minutes at $37^{\circ} \mathrm{C}$. After incubation, the phagocytic index was determined and is expressed as the percent phagocytosis of control cells. Data represent the mean \pm SEM of three independent experiments. $(*)$ indicates statistically significant difference from control group $(P<0.05)$. 

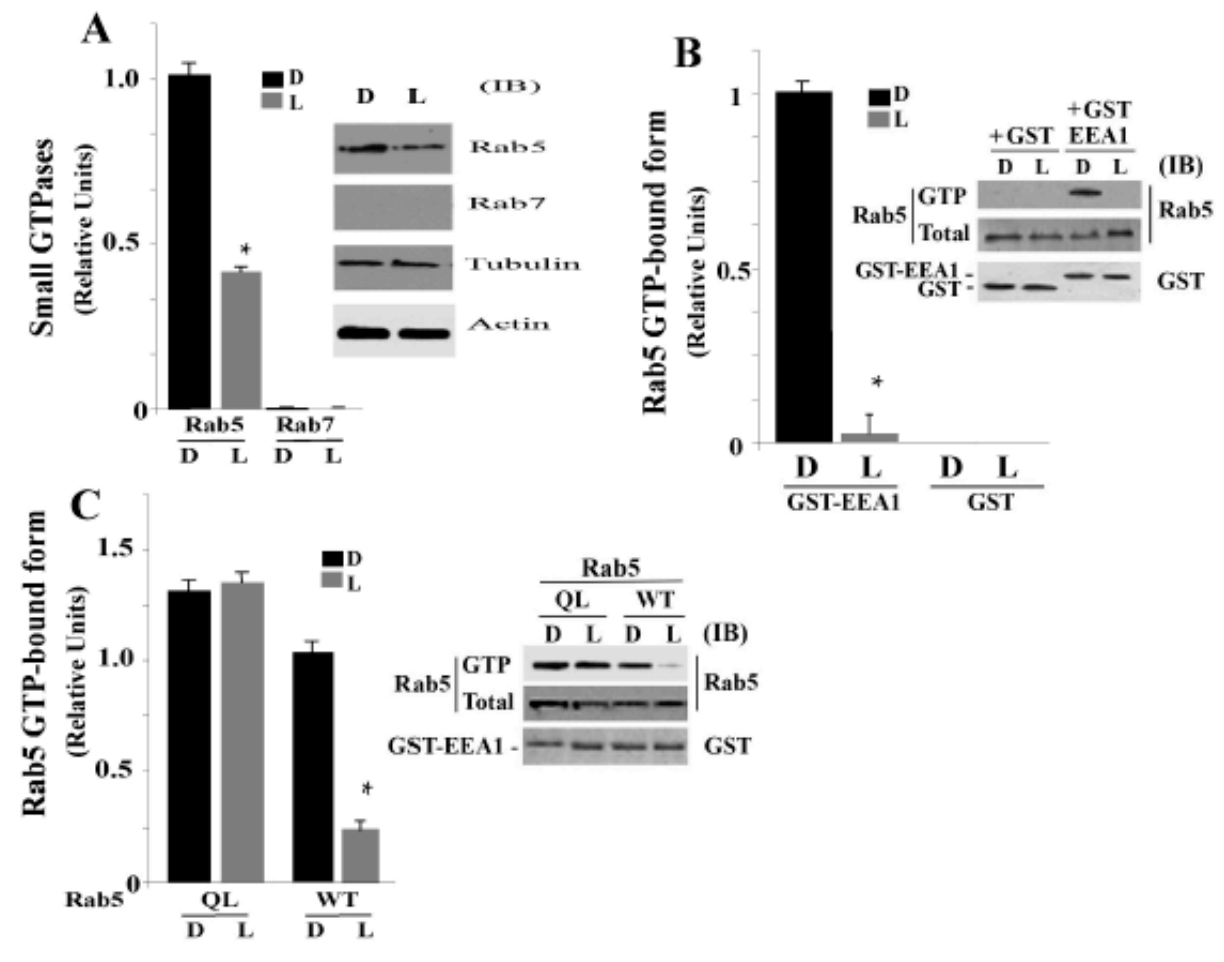

Figure 8: Live $P$. aeruginosa inhibit Rab5 activation in macrophages.

(A) J774-Eclone macrophages were incubated with live (L) or heat-inactivated (D) $P$. aeruginosa at a ratio of 200:1 for 5 minutes at $37^{\circ} \mathrm{C}$. Cells were washed, resuspended in homogenization buffer, and phagosomes containing $P$. aeruginosa were isolated, as described in Material and Methods. Phagosomal proteins were analyzed by immunoblot analysis and quantified by densitometry. Inset: Shows representative immunoblot of isolated phagosomal proteins probed with anti-Rab5, anti-Rab7, anti-tubulin or anti-actin antibodies. (B) Cells were incubated with live or heat-inactivated P. aeruginosa as in A, then washed with ice-cold PBS, lysed and incubated with glutathione beads in the presence of GST alone or GST-EEA 1 at $4{ }^{\circ} \mathrm{C}$ for $60 \mathrm{~min}$. After incubation, the beads were washed and GTP-bound activated Rab5 was analyzed by immunoblotting with anti-Rab5. Inset: Shows a representative immunoblot of samples probed with anti-Rab5 or anti-GST antibodies. (C) Cells expressing Rab5:WT or Rab5:Q79L (QL) were incubated with live or heat-inactivated $P$. aeruginosa as above, and activated Rab5 was assayed by incubating lysates with glutathione beads in the presence of GST-EEA1, followed by immunoblotting, as described in B. Inset: Shows a representative immunoblot of samples probed with anti-Rab5 or anti-GST antibodies. Data represent the mean \pm SEM of three independent experiments. $(*)$ represents statistically significant difference from control group $(P<0.05)$. 

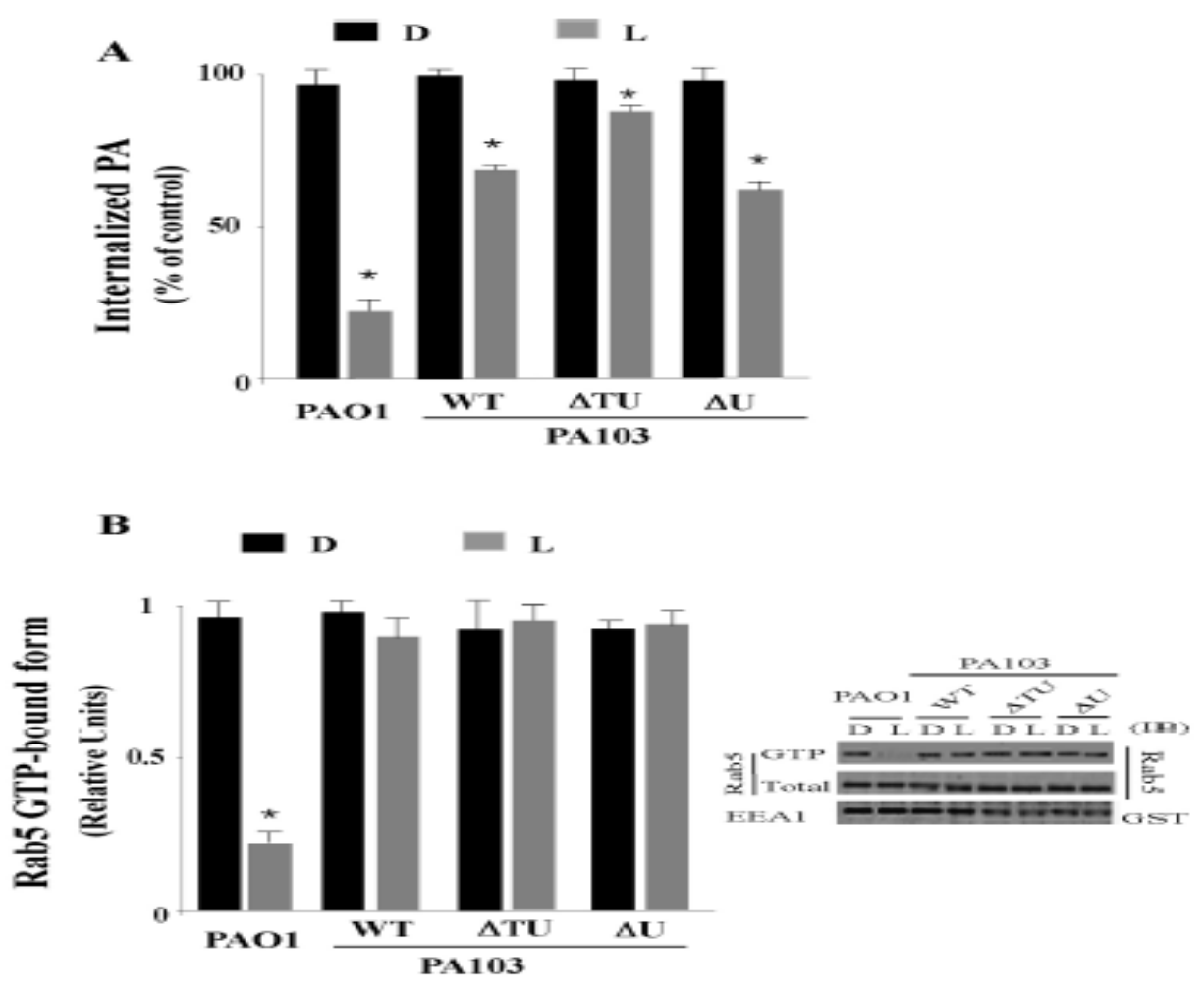

Figure 9: Selective effect $\boldsymbol{P}$. aeruginosa exotoxins on Rab5 activation in macrophages.

(A) J774-Eclone cells were incubated for 15 minutes with live (L) or heat-inactivated (D) P. aeruginosa strains: PAO1:WT, PA103:WT or PA103 lacking both ExoT and ExoU $(\Delta \mathrm{TU})$, or PA103 lacking ExoU $(\Delta \mathrm{U})$, and the phagocytic index was determined. (B) J774-Eclone cells were incubated with live or heat-inactivated $P$. aeruginosa strains as in A, and then lysed and examined for active GTP-bound Rab5 using the GST-EEA1 pulldown assay. Inset: Shows a representative immunoblot of active GTP-Rab5, and total Rab5 or GST-EEA1 in the indicated PAO1 or PA103 cell lysate. Data represent the mean \pm SEM of three independent experiments. (*) represents statistically significant difference from control group $(P<0.05)$. 


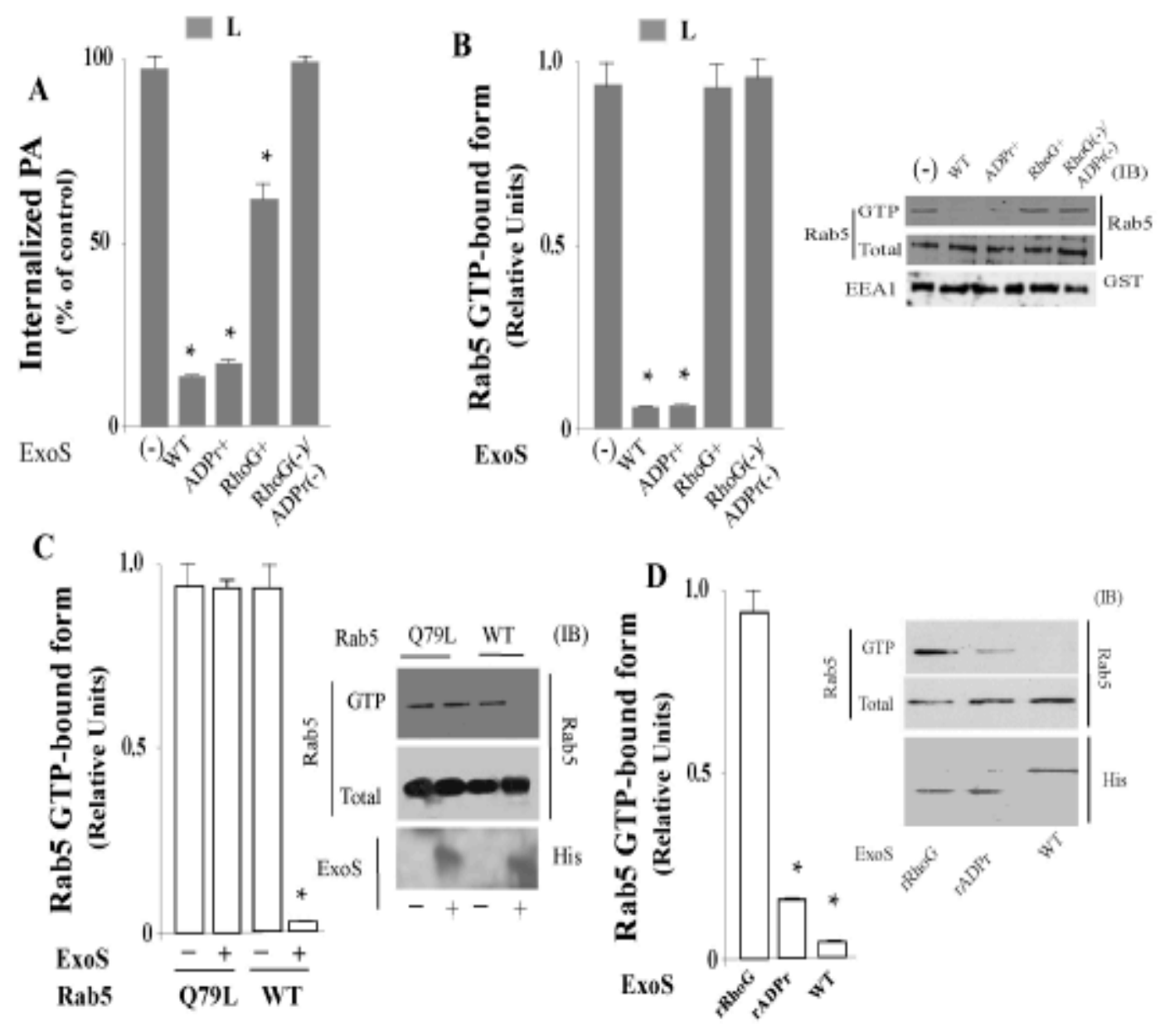

Figure 10: Effect of ExoS GAP or ADPr activity on $\boldsymbol{P}$. aeruginosa invasion.

(A) Live PA103 $\Delta \mathrm{T} \Delta \mathrm{U}$ expressing a vector control (-), ExoS (WT), an ExoS(R146A) (ADPr+) mutant, an ExoS (E379A/E387A) (RhoG+) mutant, or an ExoS (R146A/E379A/E387A) (ADPr-/RhoG-) mutant were incubated with J774-Eclone macrophages at a ratio of $200: 1$ at $37^{\circ} \mathrm{C}$ for 5 minutes. After incubation, cells were washed and the phagocytic index was determined. (B) Live PA103 strains described in Fig.A were incubated with J774-Eclone macrophages for $30 \mathrm{~min}$, and Rab5 activation 
was assayed as previously described. Inset: Shows a representative immunoblot of active GTP-Rab5, and total Rab5 or GST-EEA1 in the lysates following incubation of cells with the indicated PA103 strains. (C) Cells expressing Rab5:WT or Rab5:Q79L were transfected with 6His-ExoS (WT) (ExoS), and lysates were assayed for active Rab5 as previously described. Inset: Shows a representative immunoblot of samples probed with anti-Rab5 or anti-His-tag antibodies. (D) Cells expressing Rab5:WT were transiently transfected with 6His-ExoS (WT), 6His-ExoS (rRhoG) or 6His-ExoS (rADPr). After transfection, the activated GTP-Rab5 was determined as previously described. Inset: Shows a representative immunoblot of samples probed with anti-Rab5 or anti-His-tag antibodies. Data represent the mean \pm SEM of three independent experiments. (*) represents statistically significant difference from control group $(P<0.05)$. 

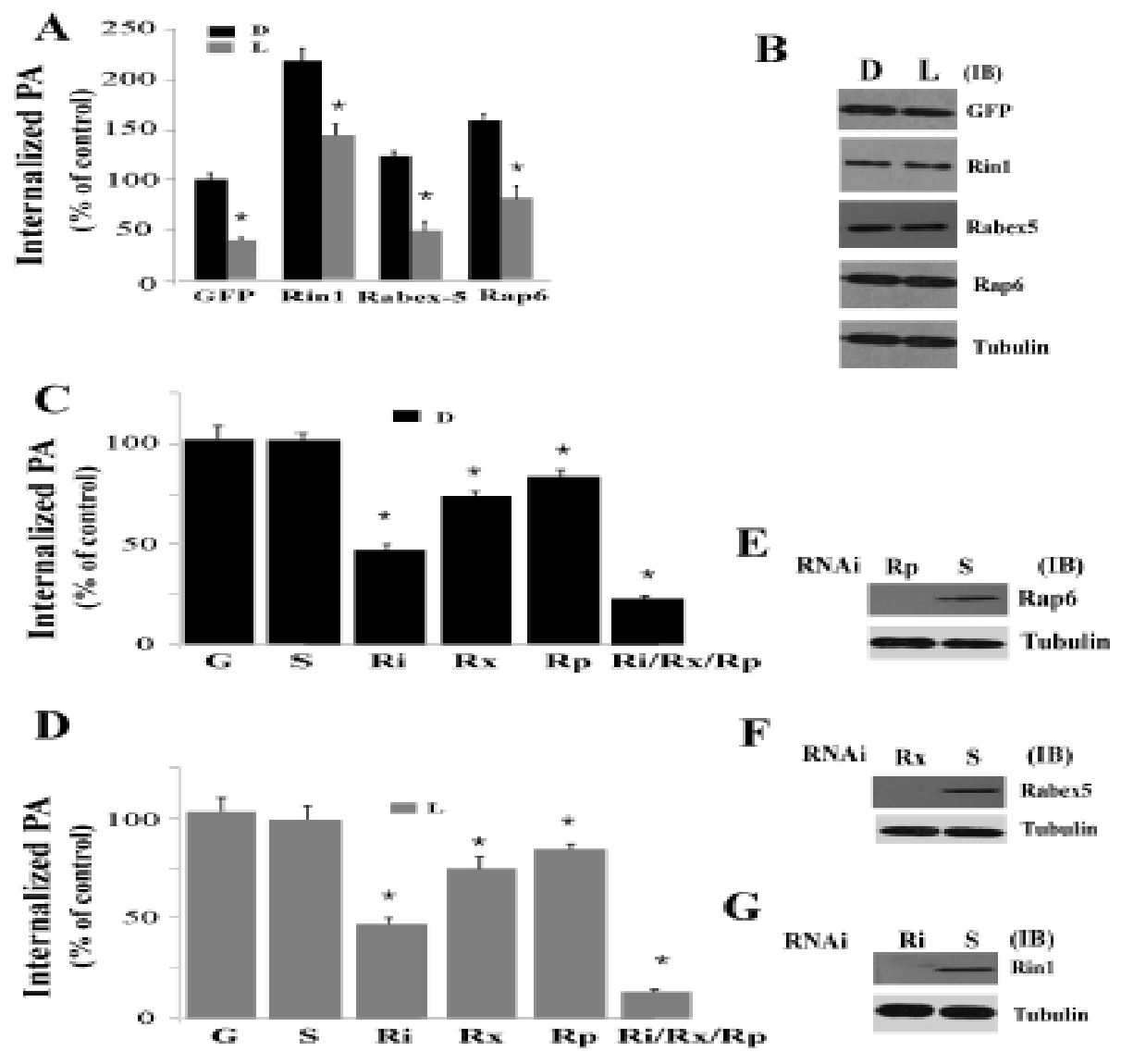

Figure 11: Effect of Rab5-GEFs in the internalization of $\boldsymbol{P}$. aeruginosa.

(A) Live (L) or heat-inactivated (D) P. aeruginosa were incubated in the presence of cells expressing GFP or the indicated Rab5-GEF at the ratio of 200:1. The phagocytic index was determined after incubation for 15 minutes at $37^{\circ} \mathrm{C}$. (B) $\mathrm{J} 774$-Eclone cells were transfected with the indicated Rab5-GEF construct, and cell lysates were immunoblotted with anti-GFP, anti-Rin1, anti-Rap6, anti-Rabex-5 or anti-tubulin antibodies. (C) Live (L) or heat-inactivated (D) P. aeruginosa were incubated with non-transfected J774-Eclone cells (G) or cells transfected with RNAi's against Rin1 (Ri), Rabex-5 (Rx), Rap6 (Rp), all three Rab5-GEFs (Ri/Rp/Rx), or scramble RNAi (S). After transfection, cells were incubated for 24 hour and then assayed for phagocytosis. (E-G) Cells were transfected with the indicated siRNAs and lysed 24 hour after post-transfection. Cell lysates were immunoblotted with anti-Rap6, anti-Rabex5, anti-Rin1 or anti-tubulin antibodies. Data represent the mean \pm SEM of three independent experiments. $(*)$ represents statistically significant difference from the control group $(P<0.05)$. 


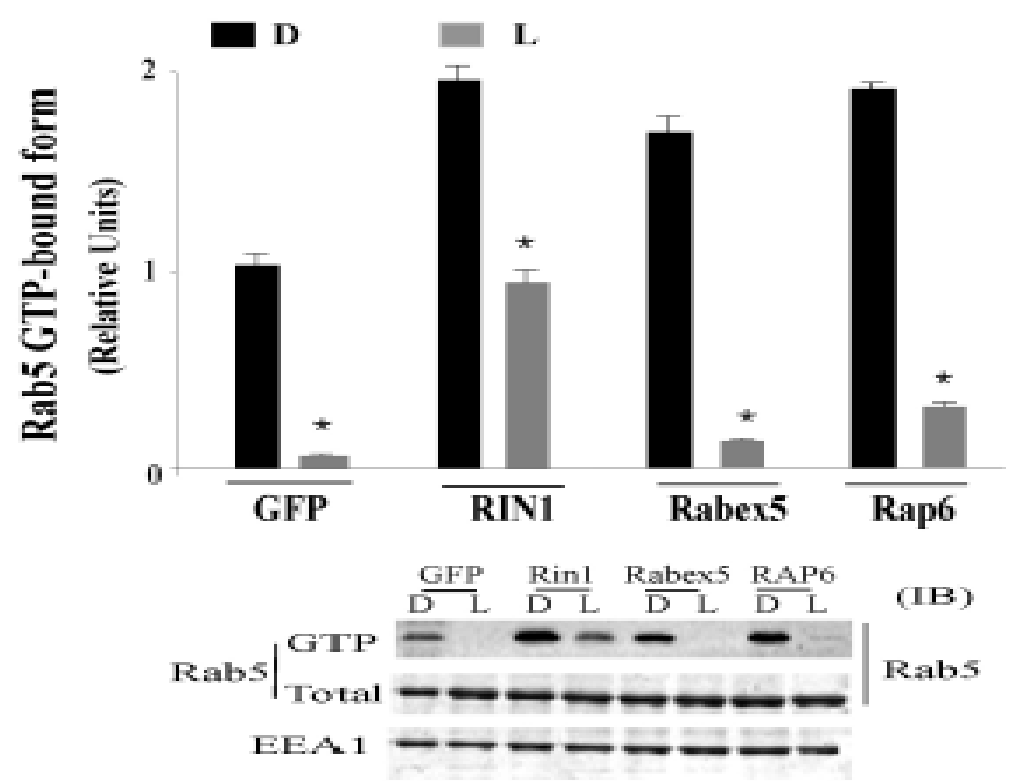

Figure 12: Effect of Rab5-GFEs on Rab5 activity during phagocytosis of $P$. aeruginosa.

Live (L) or heat-inactivated (D) $P$. aeruginosa were incubated with cells expressing GFP alone or the indicated Rab5-GEF at a ratio of 200:1. P. aeruginosa phagocytosis was assayed after incubation at $37^{\circ} \mathrm{C}$ for $5 \mathrm{~min}$. Cells were then washed and assayed for active GTP-Rab5 as previously described. A representative immunoblot of samples probed with anti-Rab5 or anti-GST is shown. Data represent the mean \pm SEM of three independent experiments. $(*)$ represents statistically significant difference from the control group $(P<0.05)$. 


\subsection{References}

Alvarez-Dominguez, C., A. M. Barbieri, W. Beron, A. Wandinger-Ness and P. D. Stahl (1996). "Phagocytosed live Listeria monocytogenes influences Rab5-regulated in vitro phagosome-endosome fusion." J Biol Chem 271(23): 13834-13843.

Angus, A. A., D. J. Evans, J. T. Barbieri and S. M. Fleiszig (2010). "The ADPribosylation domain of Pseudomonas aeruginosa ExoS is required for membrane bleb niche formation and bacterial survival within epithelial cells." Infect Immun 78(11): 4500-4510.

Barbieri, A. M., Q. Sha, P. Bette-Bobillo, P. D. Stahl and M. Vidal (2001). "ADPribosylation of Rab5 by ExoS of Pseudomonas aeruginosa affects endocytosis." Infect Immun 69(9): 5329-5334.

Barbieri, J. T. and J. Sun (2004). "Pseudomonas aeruginosa ExoS and ExoT." Rev Physiol Biochem Pharmacol 152: 79-92.

Barbieri, M. A., S. Fernandez-Pol, C. Hunker, B. H. Horazdovsky and P. D. Stahl (2004). "Role of rab5 in EGF receptor-mediated signal transduction." Eur J Cell Biol 83(6): 305314.

Bridge, D. R., K. H. Martin, E. R. Moore, W. M. Lee, J. A. Carroll, C. L. Rocha and J. C. Olson (2012). "Examining the role of actin-plasma membrane association in Pseudomonas aeruginosa infection and type III secretion translocation in migratory T24 epithelial cells." Infect Immun 80(9): 3049-3064.

Brumell, J. H. and M. A. Scidmore (2007). "Manipulation of rab GTPase function by intracellular bacterial pathogens." Microbiol Mol Biol Rev 71(4): 636-652.

Clemens, D. L., B. Y. Lee and M. A. Horwitz (2000). "Deviant expression of Rab5 on phagosomes containing the intracellular pathogens Mycobacterium tuberculosis and Legionella pneumophila is associated with altered phagosomal fate." Infect Immun 68(5): 2671-2684.

Cowell, B. A., D. Y. Chen, D. W. Frank, A. J. Vallis and S. M. Fleiszig (2000). "ExoT of cytotoxic Pseudomonas aeruginosa prevents uptake by corneal epithelial cells." Infect Immun 68(1): 403-406.

Deng, Q. and J. T. Barbieri (2008). "Modulation of host cell endocytosis by the type III cytotoxin, Pseudomonas ExoS." Traffic 9(11): 1948-1957.

Deng, Q., J. Sun and J. T. Barbieri (2005). "Uncoupling Crk signal transduction by Pseudomonas exoenzyme T." J Biol Chem 280(43): 35953-35960. 
Diaz, M. H., C. M. Shaver, J. D. King, S. Musunuri, J. A. Kazzaz and A. R. Hauser (2008). "Pseudomonas aeruginosa induces localized immunosuppression during pneumonia." Infect Immun 76(10): 4414-4421.

Diment, S., M. S. Leech and P. D. Stahl (1987). "Generation of macrophage variants with 5-azacytidine: selection for mannose receptor expression." J Leukoc Biol 42(5): 485-490.

Domingo, P., A. Ferre, M. A. Baraldes, J. Ris and F. Sanchez (1998). "Pseudomonas aeruginosa bronchopulmonary infection in patients with AIDS, with emphasis on relapsing infection." Eur Respir J 12(1): 107-112.

Duclos, S., R. Diez, J. Garin, B. Papadopoulou, A. Descoteaux, H. Stenmark and M. Desjardins (2000). "Rab5 regulates the kiss and run fusion between phagosomes and endosomes and the acquisition of phagosome leishmanicidal properties in RAW 264.7 macrophages." J Cell Sci 113 Pt 19: 3531-3541.

Fleiszig, S. M., J. P. Wiener-Kronish, H. Miyazaki, V. Vallas, K. E. Mostov, D. Kanada, T. Sawa, T. S. Yen and D. W. Frank (1997). "Pseudomonas aeruginosa-mediated cytotoxicity and invasion correlate with distinct genotypes at the loci encoding exoenzyme S." Infect Immun 65(2): 579-586.

Fraylick, J. E., M. J. Riese, T. S. Vincent, J. T. Barbieri and J. C. Olson (2002). "ADPribosylation and functional effects of Pseudomonas exoenzyme $\mathrm{S}$ on cellular RalA." Biochemistry 41(30): 9680-9687.

Fraylick, J. E., E. A. Rucks, D. M. Greene, T. S. Vincent and J. C. Olson (2002). "Eukaryotic cell determination of ExoS ADP-ribosyltransferase substrate specificity." Biochem Biophys Res Commun 291(1): 91-100.

Frithz-Lindsten, E., Y. Du, R. Rosqvist and A. Forsberg (1997). "Intracellular targeting of exoenzyme $\mathrm{S}$ of Pseudomonas aeruginosa via type III-dependent translocation induces phagocytosis resistance, cytotoxicity and disruption of actin microfilaments." Mol Microbiol 25(6): 1125-1139.

Garrity-Ryan, L., S. Shafikhani, P. Balachandran, L. Nguyen, J. Oza, T. Jakobsen, J. Sargent, X. Fang, S. Cordwell, M. A. Matthay and J. N. Engel (2004). "The ADP ribosyltransferase domain of Pseudomonas aeruginosa ExoT contributes to its biological activities." Infect Immun 72(1): 546-558.

Goehring, U. M., G. Schmidt, K. J. Pederson, K. Aktories and J. T. Barbieri (1999). "The N-terminal domain of Pseudomonas aeruginosa exoenzyme S is a GTPase-activating protein for Rho GTPases." J Biol Chem 274(51): 36369-36372.

Goh, T., W. Uchida, S. Arakawa, E. Ito, T. Dainobu, K. Ebine, M. Takeuchi, K. Sato, T. Ueda and A. Nakano (2007). "VPS9a, the common activator for two distinct types of 
Rab5 GTPases, is essential for the development of Arabidopsis thaliana." Plant Cell 19(11): 3504-3515.

Goldova, J., A. Ulrych, K. Hercik and P. Branny (2011). "A eukaryotic-type signalling system of Pseudomonas aeruginosa contributes to oxidative stress resistance, intracellular survival and virulence." BMC Genomics 12: 437.

Haas, A. K., E. Fuchs, R. Kopajtich and F. A. Barr (2005). "A GTPase-activating protein controls Rab5 function in endocytic trafficking." Nat Cell Biol 7(9): 887-893.

Hauser, A. R. (2009). "The type III secretion system of Pseudomonas aeruginosa: infection by injection." Nat Rev Microbiol 7(9): 654-665.

Hauser, A. R., S. Fleiszig, P. J. Kang, K. Mostov and J. N. Engel (1998). "Defects in type III secretion correlate with internalization of Pseudomonas aeruginosa by epithelial cells." Infect Immun 66(4): 1413-1420.

Henriksson, M. L., C. Sundin, A. L. Jansson, A. Forsberg, R. H. Palmer and B. Hallberg (2002). "Exoenzyme S shows selective ADP-ribosylation and GTPase-activating protein (GAP) activities towards small GTPases in vivo." Biochem J 367(Pt 3): 617-628.

Hueck, C. J. (1998). "Type III protein secretion systems in bacterial pathogens of animals and plants." Microbiol Mol Biol Rev 62(2): 379-433.

Hunker, C. M., A. Galvis, I. Kruk, H. Giambini, M. L. Veisaga and M. A. Barbieri (2006). "Rab5-activating protein 6, a novel endosomal protein with a role in endocytosis." Biochem Biophys Res Commun 340(3): 967-975.

Krall, R., G. Schmidt, K. Aktories and J. T. Barbieri (2000). "Pseudomonas aeruginosa ExoT is a Rho GTPase-activating protein." Infect Immun 68(10): 6066-6068.

Krall, R., J. Sun, K. J. Pederson and J. T. Barbieri (2002). "In vivo rho GTPase-activating protein activity of Pseudomonas aeruginosa cytotoxin ExoS." Infect Immun 70(1): 360367.

Li, G. and P. D. Stahl (1993). "Structure-function relationship of the small GTPase rab5." J Biol Chem 268(32): 24475-24480.

Liu, S., T. L. Yahr, D. W. Frank and J. T. Barbieri (1997). "Biochemical relationships between the 53-kilodalton (Exo53) and 49-kilodalton (ExoS) forms of exoenzyme S of Pseudomonas aeruginosa." J Bacteriol 179(5): 1609-1613.

Lyczak, J. B., C. L. Cannon and G. B. Pier (2000). "Establishment of Pseudomonas aeruginosa infection: lessons from a versatile opportunist." Microbes Infect 2(9): 10511060 . 
Madan, R., G. Krishnamurthy and A. Mukhopadhyay (2008). "SopE-mediated recruitment of host Rab5 on phagosomes inhibits Salmonella transport to lysosomes." Methods Mol Biol 445: 417-437.

Mallo, G. V., M. Espina, A. C. Smith, M. R. Terebiznik, A. Aleman, B. B. Finlay, L. E. Rameh, S. Grinstein and J. H. Brumell (2008). "SopB promotes phosphatidylinositol 3phosphate formation on Salmonella vacuoles by recruiting Rab5 and Vps34." J Cell Biol 182(4): 741-752.

Maschmeyer, G. and I. Braveny (2000). "Review of the incidence and prognosis of Pseudomonas aeruginosa infections in cancer patients in the 1990s." Eur J Clin Microbiol Infect Dis 19(12): 915-925.

McGuffie, E. M., D. W. Frank, T. S. Vincent and J. C. Olson (1998). "Modification of Ras in eukaryotic cells by Pseudomonas aeruginosa exoenzyme S." Infect Immun 66(6): 2607-2613.

Mendelson, M. H., A. Gurtman, S. Szabo, E. Neibart, B. R. Meyers, M. Policar, T. W. Cheung, D. Lillienfeld, G. Hammer, S. Reddy and et al. (1994). "Pseudomonas aeruginosa bacteremia in patients with AIDS." Clin Infect Dis 18(6): 886-895.

Mukherjee, K., S. Parashuraman, M. Raje and A. Mukhopadhyay (2001). "SopE acts as an Rab5-specific nucleotide exchange factor and recruits non-prenylated Rab5 on Salmonella-containing phagosomes to promote fusion with early endosomes." J Biol Chem 276(26): 23607-23615.

Nakaya, M., M. Tanaka, Y. Okabe, R. Hanayama and S. Nagata (2006). "Opposite effects of rho family GTPases on engulfment of apoptotic cells by macrophages." J Biol Chem 281(13): 8836-8842.

Olchowik, M. and M. Miaczynska (2009). "[Effectors of GTPase Rab5 in endocytosis and signal transduction]." Postepy Biochem 55(2): 171-180.

Pavlovskis, O. R., M. Pollack, L. T. Callahan, 3rd and B. H. Iglewski (1977). "Passive protection by antitoxin in experimental Pseudomonas aeruginosa burn infections." Infect Immun 18(3): 596-602.

Pruitt, B. A., Jr. (1974). "Infections caused by Pseudomonas species in patients with burns and in other surgical patients." J Infect Dis 130 Suppl(0): S8-13.

Rocha, C. L., J. Coburn, E. A. Rucks and J. C. Olson (2003). "Characterization of Pseudomonas aeruginosa exoenzyme $\mathrm{S}$ as a bifunctional enzyme in J774A.1 macrophages." Infect Immun 71(9): 5296-5305.

Rucks, E. A., J. E. Fraylick, L. M. Brandt, T. S. Vincent and J. C. Olson (2003). "Cell line differences in bacterially translocated ExoS ADP-ribosyltransferase substrate specificity." Microbiology 149(Pt 2): 319-331. 
Ruhen, R. W., P. G. Holt and J. M. Papadimitriou (1980). "Antiphagocytic effect of Pseudomonas aeruginosa exopolysaccharide." J Clin Pathol 33(12): 1221-1222.

Sato, H., D. W. Frank, C. J. Hillard, J. B. Feix, R. R. Pankhaniya, K. Moriyama, V. Finck-Barbancon, A. Buchaklian, M. Lei, R. M. Long, J. Wiener-Kronish and T. Sawa (2003). "The mechanism of action of the Pseudomonas aeruginosa-encoded type III cytotoxin, ExoU." EMBO J 22(12): 2959-2969.

Soong, G., D. Parker, M. Magargee and A. S. Prince (2008). "The type III toxins of Pseudomonas aeruginosa disrupt epithelial barrier function." J Bacteriol 190(8): 28142821.

Stenmark, H., R. G. Parton, O. Steele-Mortimer, A. Lutcke, J. Gruenberg and M. Zerial (1994). "Inhibition of rab5 GTPase activity stimulates membrane fusion in endocytosis." EMBO J 13(6): 1287-1296.

Sun, J. and J. T. Barbieri (2003). "Pseudomonas aeruginosa ExoT ADP-ribosylates CT10 regulator of kinase (Crk) proteins." J Biol Chem 278(35): 32794-32800.

Valencia, A., P. Chardin, A. Wittinghofer and C. Sander (1991). "The ras protein family: evolutionary tree and role of conserved amino acids." Biochemistry 30(19): 4637-4648.

Vance, R. E., A. Rietsch and J. J. Mekalanos (2005). "Role of the type III secreted exoenzymes S, T, and Y in systemic spread of Pseudomonas aeruginosa PAO1 in vivo." Infect Immun 73(3): 1706-1713.

Wretlind, B. and O. R. Pavlovskis (1983). "Pseudomonas aeruginosa elastase and its role in Pseudomonas infections." Rev Infect Dis 5 Suppl 5: S998-1004.

Yahr, T. L., A. J. Vallis, M. K. Hancock, J. T. Barbieri and D. W. Frank (1998). "ExoY, an adenylate cyclase secreted by the Pseudomonas aeruginosa type III system." Proc Natl Acad Sci U S A 95(23): 13899-13904. 


\section{Chapter 3}

Identification and characterization of ADP-ribosylation sites on Rab5 by Pseudomonas aeruginosa type III secretion protein ExoS. 


\subsection{Abstract}

Pseudomonas aeruginosa Type III secretion effector, Exoenzyme S (ExoS), is a bifunctional enzyme with ADP-ribosyl transferase activity and RhoGAP activity. Rab5 was identified as a substrate for ExoS ADP-ribosyl transferase activity both in vivo and in vitro. ExoS preferentially ADP-ribosylates arginine sites in the target proteins. To look for the specific arginine target sites on Rab5, a site directed mutagenesis technique was implied to modify all 11 Rab5 arginine sites to alanine one at a time. Modified Rab5 mutated proteins did not show any change in electrophoretic mobility or GTP-binding capacity. Although, each mutated form showed positive response toward ADPribosylation, indicating more than one ADP-ribosylation sites on Rab5. Finally, in vitro analysis also confirmed impaired GTP-binding ability in Rab5 when ADP-ribosylated, suggesting plausible conformational changes in Rab5 due to the modification.

\subsection{Introduction}

The ADP-ribosylation (Adinosine diphosphate-ribosylation) reaction is an event of post-translational protein modification leading to protein inactivation under physiological and pathological conditions. During this process, NAD (Nicotinamide adenine dinucleotide), a common respiratory co-enzyme acts as a donor of ADP-ribose moiety, the modifying group, to acceptor protein macromolecule (Hayaishi and Ueda 1977). There are four types of ADP-ribosylation modifications among which mono-ADPribosylation and poly-ADP-ribosylation (Figure 13) are mostly studied. Mono-ADPribosylation reaction is reversible and is catalyzed by an ADP-ribosyl-transferase (ADPRT) enzyme, which binds NAD and attaches the ADP-ribose moiety to a substrate protein with $\mathrm{N}$-glycosidic bond, releasing nicotinamide and a proton in the process (Ueda 
and Hayaishi 1985). Mono ADP-ribosyl transferase is found in bacterial toxins, Escheria coli protein, bacterio-phages and mammals (Koch-Nolte and Haag 1997; Corda and Di Girolamo 2003). On the other hand, poly-ADP-ribosylation is catalyzed by the enzyme Poly-ADP-ribose-synthetase/ Poly-ADP-ribose-polymerase (PARP) and is a common occurrence in the nuclei of eukaryotes (Payne and Bal 1976; Burkle and Virag 2013). In this case a chain of poly-ADP-ribose moiety linked together by ribose-ribose bond is attached to acceptor protein macromolecule (Hayaishi and Ueda 1977). Other two types of ADP-ribosylation reaction are ADP-ribose cyclization (Graeff, Liu et al. 2009) and formation of O-acetyl-ADP-ribose (Borra, O'Neill et al. 2002).

ADPR-N $+\mathrm{X} \stackrel{\text { Mono-ADP-ribosyl-transferase }}{\longrightarrow}$ ADPR-X $+\mathrm{N}+\mathrm{H}^{+}$

Poly-ADP-ribose-polymerase

$\mathrm{nADPR}-\mathrm{N}+\mathrm{X} \longrightarrow(\mathrm{ADPR}) \mathrm{n}-\mathrm{X}+\mathrm{nN}+\mathrm{n} \mathrm{H}^{+}$

\section{Figure 13: ADP-ribosylation reaction.}

Mono-ADP-ribosylation is demonstrated in equation (1) and poly-ADP-ribosylation in equation (2). NAD can be looked at as an ADP-ribose moiety covalently attached to nicotinamide by $\beta \mathrm{N}$-glycosidic linkage (ADPR-N). X represents acceptor protein, ADPR$\mathrm{X}$ is modified protein, $\mathrm{N}$ represents nicotinamide and $\mathrm{H}^{+}$represents proton and $\mathrm{n}$ as number.

Endogenous ADP-ribosylation is detected in both prokaryotes and eukaryotes (Lowery and Ludden 1988; Williamson, Smith et al. 1990). Poly-ADP-ribosylation reactions play important role in cellular signaling, transcription, DNA repair, and cell- 
cycle as well as in apoptosis and necrosis (Kreimeyer, Adamietz et al. 1985; Althaus 1992; Scovassi and Poirier 1999; Hassa, Haenni et al. 2006; Luo and Kraus 2012; Pears, Couto et al. 2012). In vertebrates, mono-ADPRT enzyme had been shown to target extracellular proteins mostly involved in immune response (Haag and Koch-Nolte 1998; Paone, Wada et al. 2002) and myogenesis (Zolkiewska and Moss 1993). On the other hand intracellular mono-ADP-ribosylation are catalyzed by several enzymes, like arginine specific mono-ADPRT enzymes which modifies $\beta$ subunit of G-proteins (Lupi, Corda et al. 2000; Lupi, Dani et al. 2002) or cellular mono-ADPRTs which generates modification of actin (Lodhi, Clift et al. 2001) and modification of elongation factor 2 (EF-2) (Fendrick and Iglewski 1989). Endogenous mono-ADP-ribosylation has been detected in prokaryotes as well (Ludden 1994). The ADP-ribosylation modification by bacterial toxins has been documented as a mechanism of pathogenesis in cholera, diphtheria, pertussis, and clostridium as well as in many other diseases (Collier 1975; Moss and Vaughan 1977; Hsia, Moss et al. 1984; Aktories, Mohr et al. 1992). A major target for bacterial ADPRT are guanyl nucleotide binding proteins involved is various cellular metabolic and regulatory pathways (Moss and Vaughan 1988), actin and EF-2 (Collier 1975; Aktories and Wegner 1989; Tezcan-Merdol, Nyman et al. 2001).

The Type III secretion effector ExoS is a bi-functional cytotoxin secreted and translocated by $P$. aeruginosa TypeIII secretion (T3S) apparatus. It was first identified by Iglewski et al (Iglewski, Sadoff et al. 1978) as an ADPRT. The amino terminus of the protein encodes Sec domain (residues 1-15) which is used to secrete the cytotoxin from the bacterium, Chap domain (residues 15-51) or chaperon binding domain which helps to maintain the extended conformation of the toxin during translocation, Membrane 
localization domain or MLD domain (residues 51-77) which targets the cytotoxin within the host cell followed by Rho GTPase-activating Protein (RhoGAP) domain (residues 96219) and ADPRT domain (residues 234-453) (Figure14) (Barbieri 2000; Deng and Barbieri 2008). Rho, Rac and Cdc42 have been established as substrates for the RhoGAP domain of ExoS both in vivo and in vitro (Goehring, Schmidt et al. 1999; Henriksson, Sundin et al. 2002; Krall, Sun et al. 2002). Several small cellular monomeric GTPases, preferably in Ras and Rab families including Ras, Rap, Ral, Rab3,4,5,6, and 7 were suggested as in vitro targets of ExoS ADPRT domain (Coburn, Dillon et al. 1989; Coburn and Gill 1991). Mammalian cytosolic 14-3-3 protein is required to activate ExoS ADPRT (Fu, Coburn et al. 1993). Sequence alignment of the catalytic domains of some major ADPRTs along with ExoS shows the presence of a conserved R-S-EXE motif with a specific affinity towards arginine residue in their target proteins (Laing, Unger et al. 2011). Studies have shown that during in vitro ADP-ribosylation of Ras by ExoS, the primary target is arginine 41 (Ganesan, Frank et al. 1998), in vivo the reflection of such modification was detected in subsequent disruption of Ras-Raf-1 signaling pathway (McGuffie, Frank et al. 1998; Ganesan, Vincent et al. 1999) although upon establishment of active Ras, the effect was reversed (Jansson, Yasmin et al. 2006). ExoS mediated ADP-ribosylation of cellular target Ral A, has been found to interfere with its respective signal transduction pathways as well (Fraylick, Riese et al. 2002). Rab5 belongs to Ras super family of GTPases and share conserved structure and biochemistry (Vetter and Wittinghofer 2001). Functional studies indicate that Rab5 is a key regulator of early phagocytosis, where it is involved in clathrin-coated vesicle formation, early phagosome fusion, phagosomal cargo recruitment and phagosomal motility (Stenmark, Parton et al. 
1994; Duclos, Diez et al. 2000; Olchowik and Miaczynska 2009). ExoS has been shown to exert antiphagocytic activity on macrophages (Frithz-Lindsten, Du et al. 1997). Previously Barbieri and others (Barbieri, Sha et al. 2001) showed that in vivo ExoS impairs intact cell function by ADP-ribosylation activity. In addition, Zhang and others has recently showed Rab5 and ExoS co-localization in target cells in vivo (Zhang, Deng et al. 2007).

In this present study, the biochemical aspect of ADP-ribosylation modification of Rab5 by ExoS has been investigated. At first arginines embedded in Rab5 protein were analyzed for their evolutionary significance according to their conserved nature in several species. All arginines in Rab5 were then mutated to alanine one at a time and then each one mutated form of Rab5 was expressed with pET28a protein expression system. Electrophoretic mobility of Rab5 mutants remained unchanged as it was confirmed by a SDS-PAGE analysis. The GTP-binding capacity of each mutated Rab5 protein was also found to be intact. When mutated Rab5 proteins were subjected to ADP-ribosylation with ExoS, each mutant were ADP-ribosylated, thus, indicating more than one arginine could be targeted during ADP-ribosyation of Rab5 protein. Finally, when ADP-ribosylated Rab5 Wild type protein (Rab5:WT) was treated with GTP, it failed to show any positive GTP-binding, which could be due to any conformational change as a possible consequence of Rab5:WT ADP-ribosylation. 


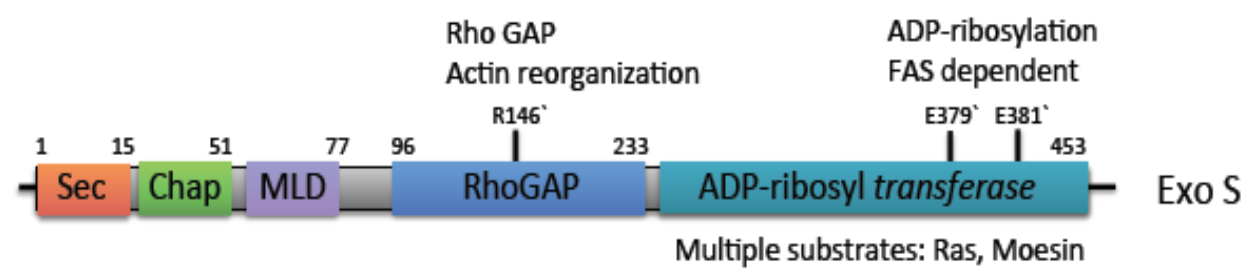

Figure 14: Schematic representations of different domains of $P$. aeruginosa ExoS.

ExoS is a bi-functional Type III secreted cytotoxin. The $\mathrm{N}$ terminus $(\mathrm{Sec})$ is used to secrete the cytotoxins from the bacterium. A chaperone binding region (Gordon, Molyneux et al. 2001) maintains the cytotoxins in an extended conformation within the bacterium poised for translocation through the Type III secretion apparatus. The membrane localization domain (MLD) targets the cytotoxins with mammalian cells for efficient modification of host proteins. ExoS possesses RhoGAP activity, inactivating Rho, Rac, and Cdc42. The ADP-ribosyltransferase domain, ExoS possess the ability to ADP-ribosylate numerous host proteins.

\subsection{Material and Methods}

\subsubsection{Materials}

All chemicals and reagents were purchased from Sigma-Aldrich (Saint Louis, MO), unless otherwise indicated. Site directed mutagenesis kit as purchased from Stratagene cloning systems (La jolla, CA). Primary and secondary antibodies used in immunoblotting were purchased from Cell Signaling Technology Inc. (Danvers, MA). 


\subsubsection{Site directed mutagenesis}

Human Rab5a gene, previously cloned into protein expression vector pET28a between HindIII and BamH1, was used as a template. Mutagenesis was performed essentially following the instructions of Stratagene cloning systems. DNA primers were constructed to be 10 base pairs complementary to the DNA template flanking the mutation of interest and encoded an arginine-to-alanine substitution (GC to $\mathrm{CG}$ ). The presence of the mutation was confirmed by sequence analysis. The mutants were designated $\mathrm{R} n \mathrm{~A}$, where arginine at residue $n(4,39,81,91,110,120,141,195,197$ and 209) of Rab5 was changed to alanine.

\subsubsection{Expression and purification of recombinant proteins}

Histidine tagged protein including Rab5 wild type and all Rab5 mutants proteins, ExoS (ExoS-pET16b, kindly provided by Michele Vidal) and Glutathione S-transeferase (Botelho, Harrison et al. 2009)-fused 14-3-3 protein (pGEXT-14-3-3), kindly provided by Dr. Phil Stahl from Washington University, St. Louis, MO) and Rab5 functional mutants Rab5:Q79L and Rab5:S34N (Mustafi, Rivero et al. 2013) were expressed in BL-21 DE3 E. coli competent cells and was purified following His-tag protein or GST-protein purification guidelines (Barbieri, Li et al. 1994; Knight and Barbieri 1997). A brief description of the purification protocol follows. Overnight cultures of E. coli BL21-DE3 carrying the appropriate pET vector or pGEX vector were diluted $1 / 20$ into LB broth containing vector specific antibiotics and shaken at $250 \mathrm{rpm}$ at $37^{\circ} \mathrm{C}$. After 2 hours, IPTG (isopropyl-b-D-thiogalactopyranoside) was added to a final concentration of $0.6 \mathrm{mM}$ and cultures were shaken for an additional 2 hours. Cultures were centrifuged at 6,000 $\mathrm{g}$ for 10 minutes, and the cell pellet of His-tag protein was suspended in binding buffer (20 
$\mathrm{mM}$ Tris- $\mathrm{HCl}$ [pH 7.9] containing $500 \mathrm{mM} \mathrm{NaCl}$ and $5 \mathrm{mM}$ imidazole) and the cell pellet of GST fusion protein in binding buffer (50 mM Tris pH 7.5, $150 \mathrm{mM} \mathrm{NaCl}, 0.05 \%$ NP40). Protease inhibitors (such as $12 \mu \mathrm{g}$ of DNase I per ml, $12 \mathrm{mg}$ of RNase A per ml, 24 $\mathrm{mg}$ of leupeptin per $\mathrm{ml}$ added in ethanol, $12 \mathrm{mg}$ of aprotinin per $\mathrm{ml}$ added in autoclaved water, and $2 \mathrm{mM}$ phenylmethylsulfonyl fluoride added in ethanol) (final concentrations) were added, and cells were broken with a French press. Cell extracts were centrifuged at $30,000 \mathrm{x} g$ for $8 \mathrm{~min}$, and the soluble material was passed through a 0.45 -mm-pore-size cellulose nitrate filter and then subjected to $\mathrm{Ni}^{+2}$-affinity chromatography (2-ml column) (Novagen, Madison,WI) or GSH chromatography (2ml column) (GE health care, Cleveland, $\mathrm{OH})$. His-tagged protein column was washed with $20 \mathrm{ml}$ of binding buffer and then with $20 \mathrm{ml}$ of binding buffer containing $50 \mathrm{mM}$ imidazole. His-tagged proteins were eluted with binding buffer containing $0.5 \mathrm{M}$ imidazole (2-ml fractions were collected). GSH column was washed with $20 \mathrm{ml}$ of $50 \mathrm{mM}$ Tris $\mathrm{pH} 8.0$ and then eluted with elution buffer (50mM Tris $\mathrm{pH} 8.0,10 \mathrm{mM}$ reduced glutathione) and $2 \mathrm{ml}$ fractions were collected. All proteins were stored at $-80^{\circ} \mathrm{C}$ with $20 \%$ glycerol until use.

\subsubsection{GTP binding assay}

One $\mu \mathrm{g}$ of purified Rab5 protein and all Rab5 mutant proteins were spotted directly onto a nitrocellulose membrane using a vacuum manifold. After 1 hour of incubation in PBS containing $0.1 \%$ tween 20 to block non-specific binding, the membrane was washed four times for 20 minutes each with PBS, $0.1 \%$ tween 20 and $5 \mathrm{mM} \mathrm{MgCl}_{2}$. GTP binding

was conducted at room temperature using $\alpha-{ }^{32} \mathrm{P}-\mathrm{GTP}(10 \mu \mathrm{Ci})$ and quantified by autoradiography. 


\subsubsection{ADP-ribosylation}

The Rab5 ADP-ribosylation was determined by using biotinylated NAD (Zhang 1997). The reaction was carried out essentially as previously described with few modifications (Barbieri, Tall et al. 2001; Fraylick, Rucks et al. 2002). Briefly a 50 $\mu 1$ reaction mixture of His tagged Rab5 (one individual mutant), His-Rab5 Q79L or His Rab5 S34N protein $(1 \mu \mathrm{g})$ along with His-ExoS $(0.8 \mu \mathrm{M}), \mathrm{GST}-14-3-3(0.8 \mu \mathrm{M}), 0.2 \mathrm{M} \mathrm{Na}$ acetate ( $\mathrm{pH}$ 6.0), 1mM Magnesium chloride and $10 \mathrm{mM}$ Biotinylated NAD (8-([Nbiotinyl(6-aminohexyl)]amino)NAD) (Sigma) was incubated at $25^{\circ} \mathrm{C}$ for 1 hours. For SDS-PAGE analysis, reaction was stopped by adding 4x leammli sample buffer and boiling for 5 minutes at $95^{\circ} \mathrm{C}$. For dotblot analysis, reaction was halted by a quick chill over ice.

\subsubsection{Protein analysis}

For immunoblotting, protein samples were boiled in Leammli buffer (60 mM Tris$\mathrm{HCl}, 1 \%(\mathrm{w} / \mathrm{v}) \mathrm{SDS}, 10 \%$ glycerol, $0.05 \%(\mathrm{w} / \mathrm{v})$ bromophenol blue, $\mathrm{pH} 6.8$, with $2 \% \beta$ mercaptoethanol). Samples were subjected to $10 \%$ or $12 \%$ SDS-PAGE and transferred to nitrocellulose membranes for immunoblotting. Nitrocellulose membranes were incubated with blocking solution (TBS containing $0.1 \%$ Tween 20 and 5\% BSA) and were probed with the indicated antibodies. For Dot Blot, protein samples were spotted onto nitrocellulose membrane embedded inside dot blot ensemble (Abcam, San Francisco, CA) and incubated with blocking solution (TBS containing 0.1\% Tween 20 and 5\% BSA) and were probed with HRP-avidin. 


\subsubsection{Bioinformatics}

The Rab5 mRNA sequences of from several species such as Leishmania donovani (AAR03593.1), Toxoplasma gondii (AAG10794.1), Arabidopsis thaliana (AEE84207.1), Anopheles gambiae (ABL74413.1), Homo sapiens (AAB08927.1) and Mus musculus (NP_080163.1) were obtained from NCBI GenBank and analyzed for homology using multiple sequence alignment tool "Clustal Omega". Regions of similarity indicated evolutionary conserved motif with structural or functional importance.

\subsubsection{Image quantification}

The NIH Image J64 was used to quantify Western blots after images were scanned at a grayscale amplification of 600 dpi. Digital images of the Western blot from cell lines were captured and loaded into ImageJ64, and-Rab5 specific bands, along with $\alpha$ tubulin bands, were assessed in each sample using the Analyze $\rightarrow$ Gels function, which allows for background correction. The ratio of Rab5 signal to $\alpha$ tubulin was calculated for each sample and served as an index of Rab5 expression. The index of expression for other proteins examined in this study was derived in a similar manner.

\subsubsection{Statistical analysis}

All samples in this study were analyzed in duplicate and each experiment was repeated three times. Values represent the mean \pm SEM of three independent experiments. To compare two groups, student's t test was used. $P<0.05$ was considered as statistically significant. 


\subsection{Results}

\subsubsection{Three arginine sites in Rab5 are conserved implying their importance in Rab5}

\section{structure.}

Arginines are preferred target sites on a substrate protein for ExoS ADP-ribosylation activity. Rab5 protein structure holds eleven arginines. Through a species wise multiple sequence alignment (Figure 15), three arginine sites at position 39, 81 and 91 were found to be conserved in Rab5. Arginine-81 and arginine-91 resides in a conserved region of Rab5 structure also known as Switch II region. Arginine-4 and arginine- 8 are part of the $\mathrm{N}$ terminal region in Rab5 responsible for geranylgeranylation of its $\mathrm{C}$ terminal Cystein (Sanford, Pan et al. 1995). Arginine-195 and arginine-197 lies within an RXRGVDLXE motif in Rab5 which is important in membrane targeting (Bucci, Lutcke et al. 1995).

\subsubsection{Preparation and purification of several single arginine mutated Rab5.}

As multiple sequence analysis suggested the significance of some individual position of arginine molecules embedded in Rab5, it was tempting to perform an over all analysis of each individual site as possible ADP-ribosylation target. Human-Rab5a DNA cloned into $\mathrm{pET} 28 \mathrm{a}$ protein expression vector was subjected to site directed mutagenesis. 11 individual arginine to alanine mutations (each mutant was designated RnA (Arginine at residue $\mathrm{n}$ of Rab5 mutated to an alanine) (Figure 16) were introduced into the Rab5 open reading frame following a previously established study (Ganesan, Frank et al. 1998). Each mutated form of Rab5 plasmids were introduced into E.coli (BL21-DE3) competent cells for protein expression. No change in viability of the transformed E.coli cells confirmed absence of lethal effect as results of the Rab5 mustations (data not shown). 


\subsubsection{Modification of recombinant Rab5 protein didn't disrupt electrophoretic mobility.}

The His-tagged Rab5:WT, His-tagged Rab5:Q79L, His-tagged Rab5:S34N and all Rab5 mutants plasmids transformed in BL21-DE3-E.coli cells were grown in Lauria broth (LB) at $37^{\circ} \mathrm{C}$ and induced with $1 \mathrm{mM}$ IPTG for 3 hours. Pellets were collected and lysed with lysozyme, DNAse, protease inhibitors in His-specific buffer and run through affinity chromatography columns to obtain purified proteins which were afterwards subjected to SDS-PAGE gel elctrophoresis. No significant change in electrophoretic mobility was observed among the mutated Rab5 protein because of amino acid substitution (Figure 17).

\subsubsection{GTP-binding ability among mutated Rab5 protein.}

Since arginine is positively charged amino acid and alanine is negatively charged, substitution of the earlier with the later could disrupt several activity of the protein. Although, specific functional role of arginines in Rab5 has not yet been predicted, it was important to observe the GTP-binding ability among mutated Rab5 proteins. Rab5 $(1 \mu \mathrm{g})$ protein along all Rab5 mutant proteins were spotted directly onto a nitrocellulose membrane by dot blot technique and blocked with PBS, $0.1 \%$ tween 20 for $20 \mathrm{~min}$. the membrane was then washed with PBS, $0.1 \%$ tween 20 and 5mM Magnesium chloride, 3

times, 20 minutes each. GTP-binding was conducted at room temperature using $\alpha-{ }^{32} \mathrm{P}$ radio labeled-GTP and quantified by autoradiography (Figure 18). Structural composition of Rab5:S34N protein does not support GTP binding where as Rab5:Q79L structure is favorable for GTP binding. Hence, as a control, Rab5:S34N and Rab5:Q79L purified 
proteins were subjected to GTP binding as well. Autoradiography results showed no significant disruption of GTP binding among mutated Rab5 proteins.

\subsubsection{ADP-ribosylation status of arginine mutated Rab5.}

In order to investigate the specific arginine in Rab5 protein that could be targeted during ADP-ribosylation, each Rab5 mutant protein along with Rab5:WT protein was subjected to ADP-ribosylation with purified ExoS protein. The goal of this study was to observe less or no ADP-ribosylation in at least one of the mutant protein because of the effect of substitution. The Rab5 ADP-ribosylation status was determined by using a novel technique where biotinylated NAD was used as an ADP-ribose donor and later detected by biotin-HRP-avidin reaction. Purified ExoS in presence of 14-3-3 was incubated with Rab5 and mutant proteins strictly following the established protocol (Ganesan, Frank et al. 1998). Despite mutation in one particular arginine in each Rab5 mutant, all proteins were positive for ADP-ribosylation (Figure 19). This provided the information that ADPribosylation target on Rab5 is possibly more than one arginine site rather than one.

\subsubsection{Discrepancies in Rab5-GTP binding post ADP-ribosylation modification}

Finally, the effect of Rab5 ADP-ribosylation modification on GTP-binding was examined. Rab5:WT (ADP-ribosylated or not) was immobilized on a nitrocellulose membrane using Dot blot technique. The membrane was washed as previously described (Materials and Methods) and blocked with $5 \mathrm{mM}$ magnesium chloride essentially as mentioned before. A $\alpha-{ }^{32} \mathrm{P}$ radio labeled-GTP was added on top the membrane and the GTP incorporation was visualized by autoradiograph. Modified or un-modified Rab5:S34N and Rab5:Q79L were used as negative and positive controls respectively. Results showed least or no GTP incorporation in ADP-ribosylated Rab5:WT, where as 
Rab5:Q79L in both form remained capable for GTP-binding (Figure 20). As expected, Rab5:S34N did not show any GTP-binding irrespective of its ADP-ribosylation form or normal form.

\subsection{Discussion}

With the recognition of the role of ExoS ADPRT activity on modulation of function (Barbieri 2000; Deng and Barbieri 2008; Mustafi, Rivero et al. 2013) and altered electrophoretic mobility of Rab5 protein (Fraylick, Rucks et al. 2002), it directed the attention to pursue a biochemical analysis of the nature of Rab5 ADP-ribosylation. Ras has been shown to be ADP-ribosylated by bacterially translocated ExoS at more than one sites including arginine-41 (Ganesan, Frank et al. 1998), and RalA, another Ras family protein, is efficiently ADP-ribosylated at arginine-52 (Fraylick, Riese et al. 2002) in eukaryotic cells. Rab5 protein belongs to a large family of Ras related GTPase protein and is a ubiquitous regulator of intracellular vesicle transport. Human Rab5a, a 215 amino acid long protein contains 11 arginine sites as a potential target for ExoS ADPRT activity. Three of these arginine sites are conserved among Rab5 in several species (Figure 15). Another interesting fact is that arginine-81 and arginine-91 lies within the conserved "switch II" domain of Rab5 (Figure 16). In the present study, an individual mutation analysis was implied for each of the arginines. With the help of site directed mutagenesis technique, each arginines in Rab5 were individually mutated to alanine and the modified Rab5 was expressed in His-tagged expression vector (pET28a) (Figure 17). Further, a GTP-binding analysis confirmed that each of the mutated Rab5 did not lose their structural conformation for recognition and binding to GTP molecules. Both in vitro and in vivo ADP-ribosylation that has been studied by Fraylick and others (Fraylick, 
Rucks et al. 2002) showed the altered mobility of ADP-ribosylated-Rab5 when subjected to electrophoresis, which was re established in this study (Figure 18A). Here, a new approach was also adopted in order to label and confirm ADP-ribosylation events in Rab5. Biotinylated NAD was used as an ADP-ribose donor to Rab5 arginine/arginines in presence of ExoS ADPRT activity, which was then detected on nitrocellulose membrane using HRP-Avidin probe. Along with Rab5:WT, Rab5:S34N and Rab5:Q79L, all Rab5 mutants were positive for ADP-ribosylation modification (Figure 18B). The result strongly indicates that more than one preferred arginine sites could be ADP-ribosylated. Finally, it was interesting to observe the effect of ADP-ribosylation modification of Rab5 on in vitro GTP-binding ability. Rab5:Q79L (a GTP dissociation defective mutant) showed positive GTP binding whether ADP-ribosylated or not (Figure 19). Similarly as expected, Rab5:S34N (GDP dissociation defective mutant) did not show GTP-binding in both ADP-ribosylated and normal state (Figure 19). Also, when Rab5:WT, whether previously ADP-ribosylated or not, subjected to in vitro GTP-binding, the ADPribosylated form failed to show positive signal for the reaction. Taken together, these data suggested a possible effect of ADP-ribosylation modification on Rab5 leading to disruption in GTP binding ability.

On the basis of study by Nikolova et al. (1998), the most exposed sites for proteolysis in Rab5 are arginine-197, arginine-4 and arginine-81. Arginine-197 lies within an RXRGVDLXE motif in Rab5 which is important in membrane targeting (Bucci, Lutcke et al. 1995). Arginine-4 the second point of proteolysis on Rab5 lies with the first 20 residue of Rab5, loss of which ceases the ability of the protein's endocytic activity in vitro ( $\mathrm{Li}$ and Stahl 1993). The third point of proteolysis is arginine-81, which lies within 
the sequence that entails specific protein-protein interaction. In addition, mutating Rab5 with R81A showed decrease in endosome fusion and as a second mutation in Rab5 N133I, R81A showed complete prevention of prenylation (Li, Barbieri et al. 1994). Also as suggested by other studies, the preferred site of ADP-ribosylation in Ras is arginine-41 and in RalA is arginine-52 which is adjacent to "Switch I" region and advent in effector interaction (Ganesan, Frank et al. 1998; Fraylick, Rucks et al. 2002). Arginine-39 in Rab5 is the equivalent site in Rab5 same as Ras arginine-41 and Ral arginine-52, hence could be a possible target. Over all, several essential arginine sites in Rab5 could bring in the impaired activity either through disrupted Guanine exchange factor interaction or inhibiting effector protein binding. More than one ADP-ribose moiety can change the structural confirmation and modify substrate accessibility. A detailed atomic spectral analysis of ADP-ribosylated protein awaits further information on the specificity of target arginine in Rab5 and the nature of odification. 


\subsection{Figure Legands}

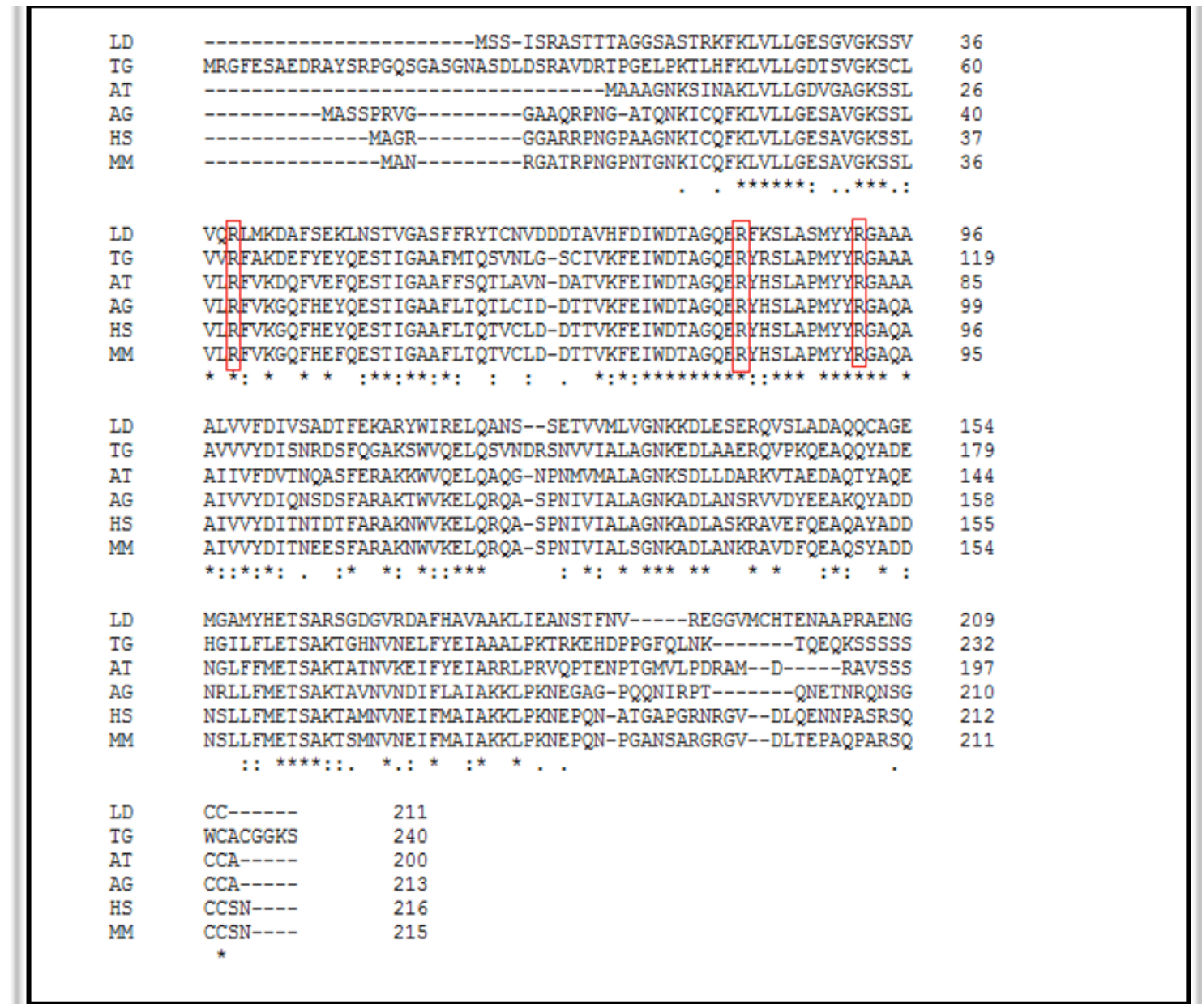

Figure 15: Rab5 multiple sequence alignment.

Multiple sequence alignment of Rab5 mRNA from Leishmania donovani (LD), Toxoplasma gondii (TG), Arabidopsis thaliana (AT), Anopheles gambiae (AG), Homo sapiens (HS) and Mus musculus (MM). Arginine at position 39, 81 and 91 (HS) were found to be conserved in Rab5 among these species. 


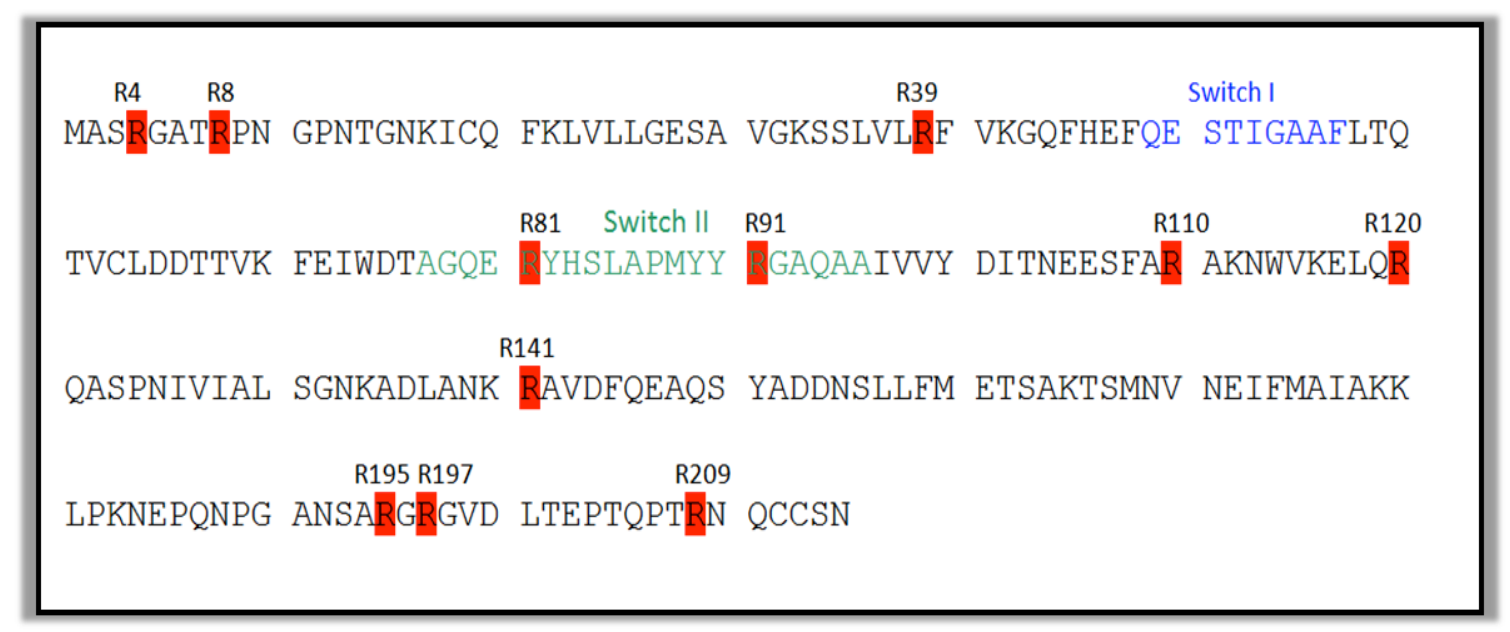

Figure 16: Arginine mutation sites on Rab5.

All indicated arginines in Rab5 were substituted with alanine as described in Materials and Methods. 11 forms of arginine-mutated Rab5 plasmid named (RnA) R4A, R8A, R39A, R81A, R91A, R110A, R120A, R141A, R195A, R197A and R209A carried by pET28a expression vector were then used for several experiments for this study. 


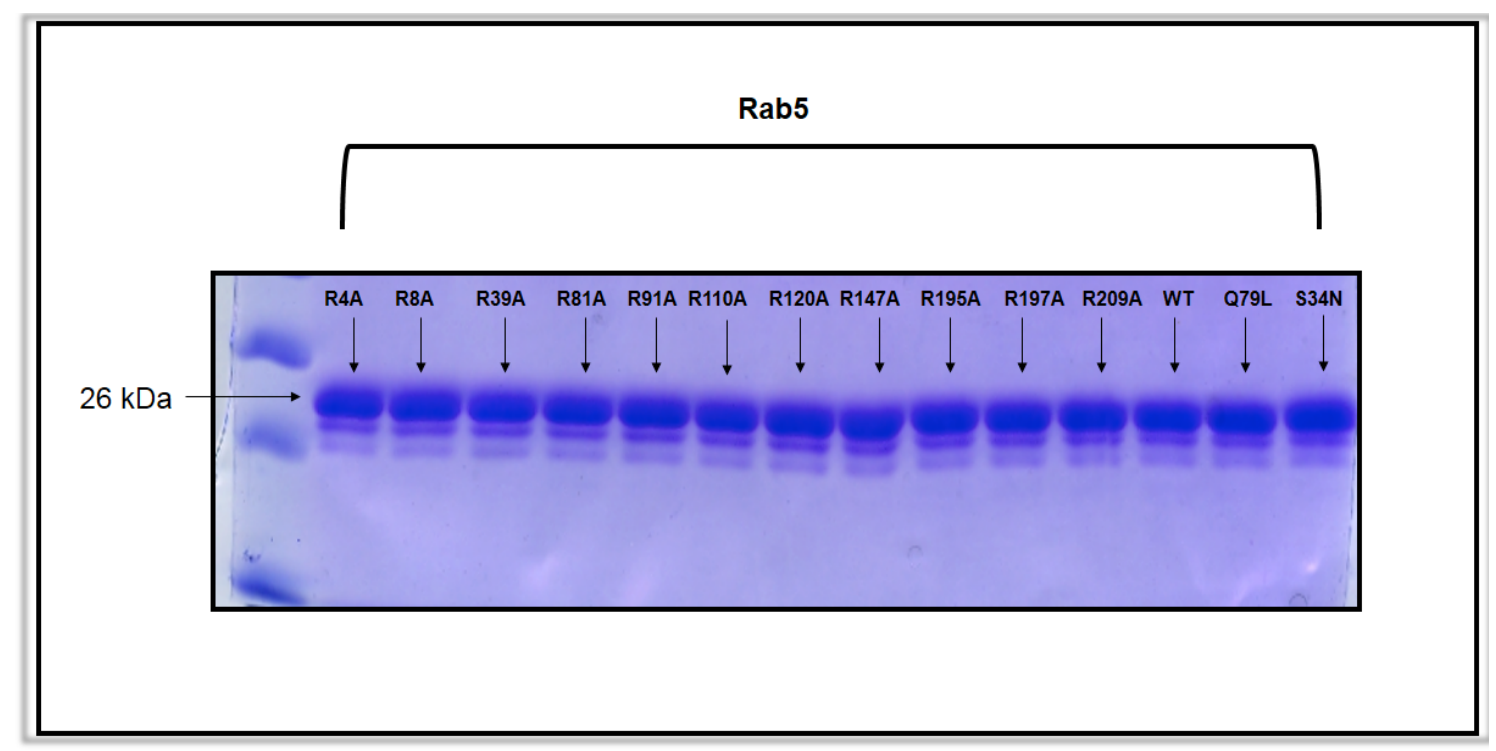

Figure 17: Purification of mutated Rab5 proteins.

All indicated arginines in Rab5 were substituted with alanine as described earlier. Each modified Rab5 were then expressed in E.coli expression system with pET28a expression vector. Following a His-tag protein purification protocol, 11 forms (RnA) of Arg-mutated Rab5 along with Rab5:Q79L (pET28a) and Rab5:S34N (pET28a) were purified and approximately $0.5 \mu \mathrm{g}$ of purified Rab5 protein boiled in protein sample buffer was subjected to $12 \%$ SDS-PAGE and stained with Coomassie bleu. SDS-GEL analysis indicated all proteins purified consist of $99 \%$ of $26 \mathrm{kDa}$ Rab5 protein. The results are representative of analysis performed in 3 independent experiments. 


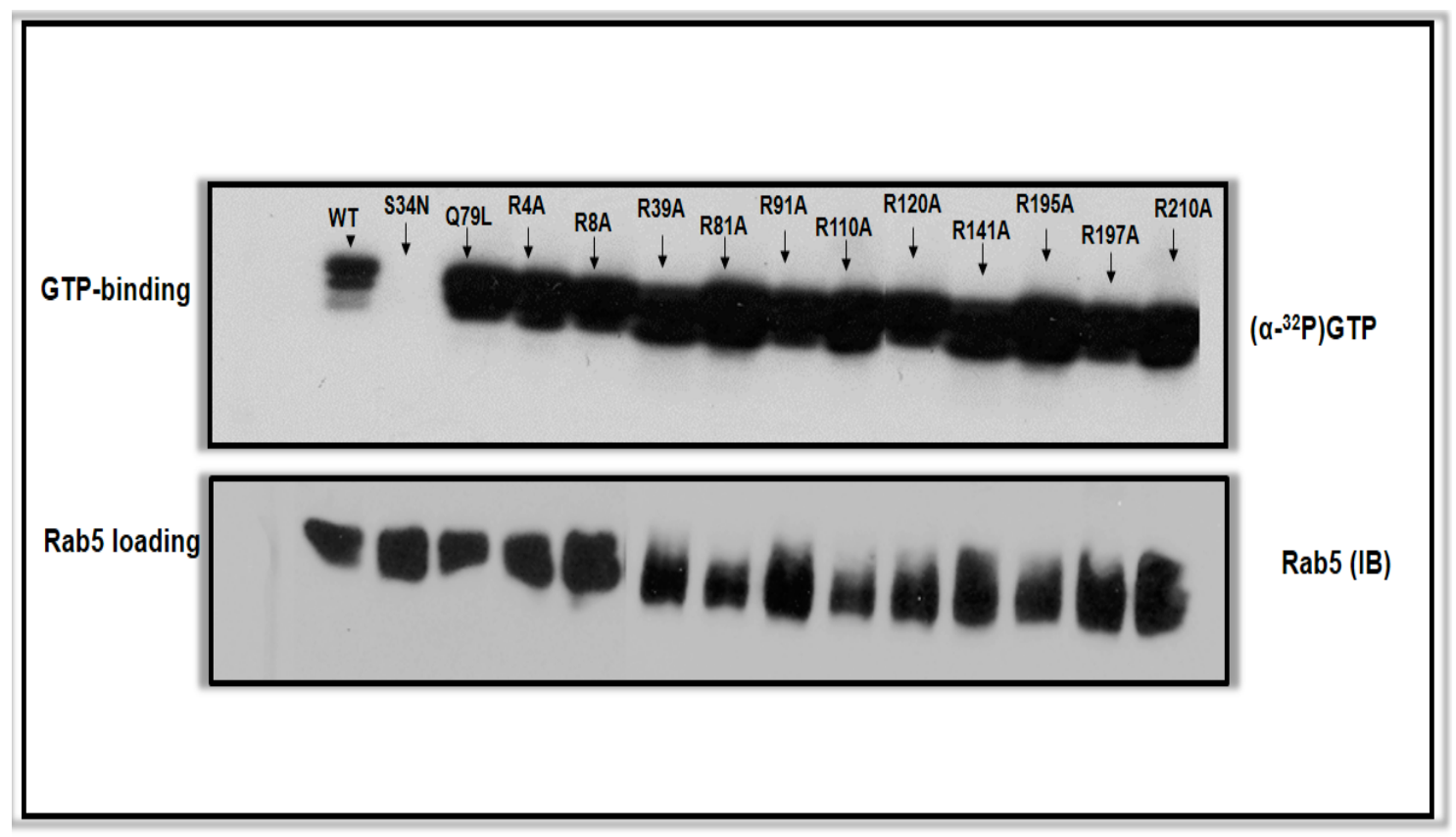

Figure 18: GTP binding analysis of Rab5 mutants.

All Rab5 proteins purified for this study including wildtype (WT), Rab5:Q79L (Q79), Rab5:S34N (S34N) along with 11 single arginine mutant forms (RnA) were analyzed for their ability to bind with GTP as described in material methods section. Radiolabeled $(\alpha-$ $\left.{ }^{32} \mathrm{P}\right)$-GTP $(10 \mu \mathrm{Ci})$ were added to a nitrocellulose membrane previously spotted with several Rab5 proteins and GTP binding signal was captured by autoradiography technique as demonstrated on the top panel. All purified proteins as mentioned above shows normal GTP binding except for Rab5:S34N, which is a constitutively negative mutant. Bottom panel represents loading control of all proteins as probed in immunoblot. The results are representative of analysis performed in 3 independent experiments. 


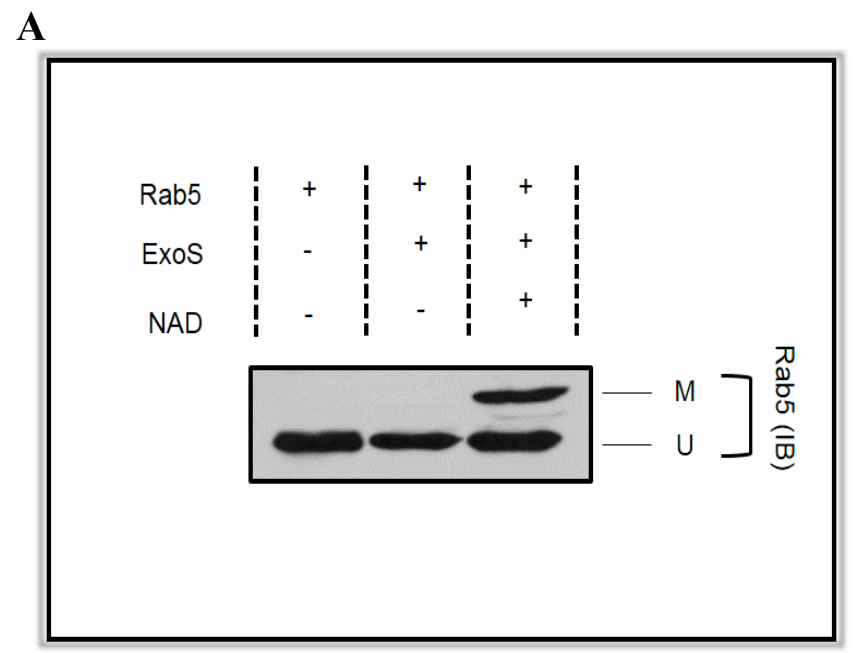

\section{B}

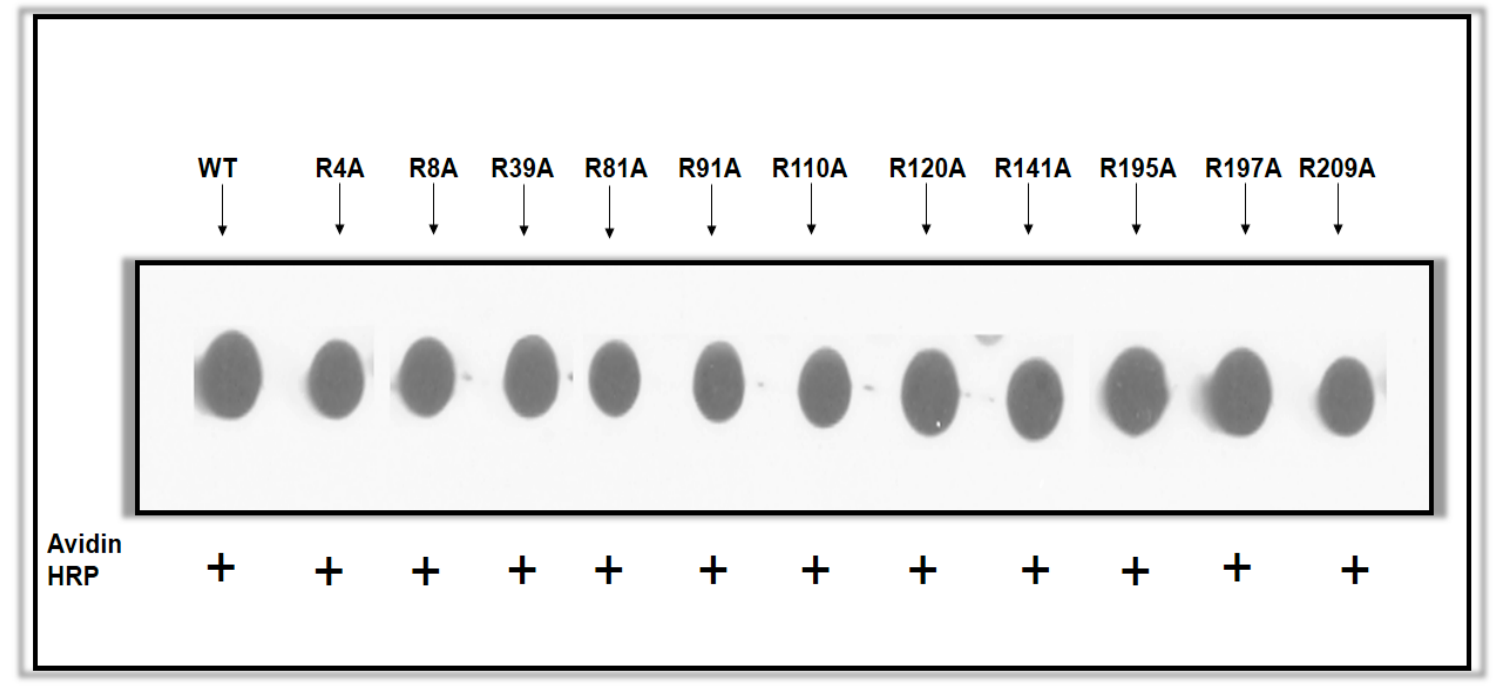

Figure 19: ADP-ribosylation analysis.

(A) Rab5: WT protein was ADP-ribosylated by ExoS as described in Material and Methods and analyzed on a 12\% SDS-PAGE. Change in Elecrtrophoretic shift was observed in ADP-ribosylated Rab5 (M) when compared to Unmodified (U) Rab5. (B) ADP-ribosylation was detected using HRP-avidin applied to nitrocellulose membrane previously spotted with ADP-ribosylated Rab5 mutants along with Rab5:WT (WT). Along with WT, all mutants were positive for ADP-ribosylation implying that more than one arginine is targeted masking the unmodified single mutant in each form. The results are representative of analysis performed in 3 independent experiments. 


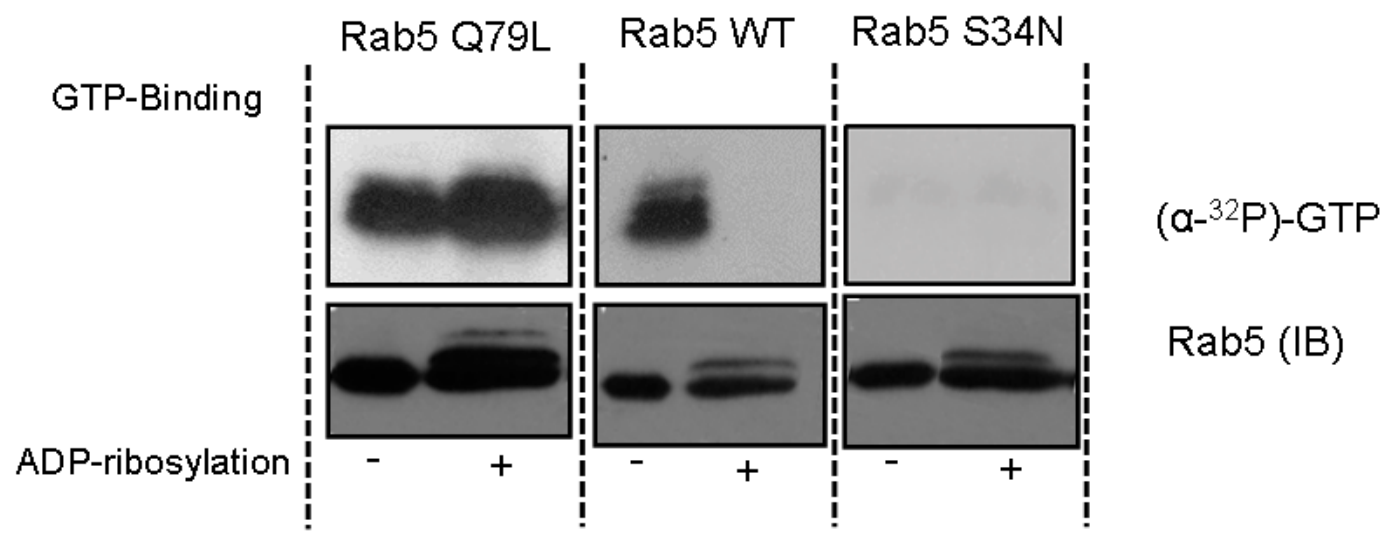

Figure 20: GTP binding status of ADP-ribosylated Rab5.

Rab5:WT, Rab5:S34N and Rab5:Q79L were subjected to ADP-ribosylation and confirmed with immunoblot using Rab5 antibody (bottom panel). Afterwards, ADPribosylated proteins were spotted on a nitrocellulose membrane using dot blot and incubated with $10 \mu \mathrm{Ci}$ of radiolabeled ${ }^{32} \mathrm{P}-\mathrm{GTP}$. GTP-binding ability was analyzed in both modified and unmodified Rab5 (7 top panel). Constitutively active Rab5:Q79L was used as a positive control showing successful GTP binding in both form, and Rab5:S34N, a negative control showed no GTP binding activity. Rab5:WT, when modified by ADPribosylation, shows loss of GTP binding capacity compared to unmodified Rab5:WT form. The results are representative of analysis performed in 3 independent experiments. 


\subsection{References}

Aktories, K., C. Mohr and G. Koch (1992). "Clostridium botulinum C3 ADPribosyltransferase." Curr Top Microbiol Immunol 175: 115-131.

Aktories, K. and A. Wegner (1989). "ADP-ribosylation of actin by clostridial toxins." J Cell Biol 109(4 Pt 1): 1385-1387.

Althaus, F. R. (1992). "Poly ADP-ribosylation: a histone shuttle mechanism in DNA excision repair." J Cell Sci 102 ( Pt 4): 663-670.

Barbieri, A. M., Q. Sha, P. Bette-Bobillo, P. D. Stahl and M. Vidal (2001). "ADPribosylation of Rab5 by ExoS of Pseudomonas aeruginosa affects endocytosis." Infect Immun 69(9): 5329-5334.

Barbieri, J. T. (2000). "Pseudomonas aeruginosa exoenzyme S, a bifunctional type-III secreted cytotoxin." Int J Med Microbiol 290(4-5): 381-387.

Barbieri, M. A., G. Li, M. I. Colombo and P. D. Stahl (1994). "Rab5, an early acting endosomal GTPase, supports in vitro endosome fusion without GTP hydrolysis." J Biol Chem 269(29): 18720-18722.

Barbieri, M. A., G. G. Tall, B. F. Horazdovsky and P. D. Stahl (2001). "Receptormediated endocytosis: EGF/Ras-activated endocytosis is mediated by Rab5 and the Rab5 guanine nucleotide exchange activity of RIN1." Faseb Journal 15(5): A1162-A1162.

Borra, M. T., F. J. O'Neill, M. D. Jackson, B. Marshall, E. Verdin, K. R. Foltz and J. M. Denu (2002). "Conserved enzymatic production and biological effect of O-acetyl-ADPribose by silent information regulator 2-like NAD+-dependent deacetylases." J Biol Chem 277(15): 12632-12641.

Botelho, R. J., R. E. Harrison, J. C. Stone, J. F. Hancock, M. R. Philips, J. Jongstra-Bilen, D. Mason, J. Plumb, M. R. Gold and S. Grinstein (2009). "Localized diacylglyceroldependent stimulation of Ras and Rap1 during phagocytosis." J Biol Chem 284(42): $28522-28532$.

Bucci, C., A. Lutcke, O. Steele-Mortimer, V. M. Olkkonen, P. Dupree, M. Chiariello, C. B. Bruni, K. Simons and M. Zerial (1995). "Co-operative regulation of endocytosis by three Rab5 isoforms." FEBS Lett 366(1): 65-71.

Burkle, A. and L. Virag (2013). "Poly(ADP-ribose): PARadigms and PARadoxes." Mol Aspects Med.

Coburn, J., S. T. Dillon, B. H. Iglewski and D. M. Gill (1989). "Exoenzyme S of Pseudomonas aeruginosa ADP-ribosylates the intermediate filament protein vimentin." Infect Immun 57(3): 996-998. 
Coburn, J. and D. M. Gill (1991). "ADP-ribosylation of p21ras and related proteins by Pseudomonas aeruginosa exoenzyme S." Infect Immun 59(11): 4259-4262.

Collier, R. J. (1975). "Diphtheria toxin: mode of action and structure." Bacteriol Rev 39(1): 54-85.

Corda, D. and M. Di Girolamo (2003). "Functional aspects of protein mono-ADPribosylation." EMBO J 22(9): 1953-1958.

Deng, Q. and J. T. Barbieri (2008). "Molecular mechanisms of the cytotoxicity of ADPribosylating toxins." Annu Rev Microbiol 62: 271-288.

Duclos, S., R. Diez, J. Garin, B. Papadopoulou, A. Descoteaux, H. Stenmark and M. Desjardins (2000). "Rab5 regulates the kiss and run fusion between phagosomes and endosomes and the acquisition of phagosome leishmanicidal properties in RAW 264.7 macrophages." J Cell Sci 113 Pt 19: 3531-3541.

Fendrick, J. L. and W. J. Iglewski (1989). "Endogenous ADP-ribosylation of elongation factor 2 in polyoma virus-transformed baby hamster kidney cells." Proc Natl Acad Sci U S A 86(2): 554-557.

Fraylick, J. E., M. J. Riese, T. S. Vincent, J. T. Barbieri and J. C. Olson (2002). "ADPribosylation and functional effects of Pseudomonas exoenzyme S on cellular RalA." Biochemistry 41(30): 9680-9687.

Fraylick, J. E., E. A. Rucks, D. M. Greene, T. S. Vincent and J. C. Olson (2002). "Eukaryotic cell determination of ExoS ADP-ribosyltransferase substrate specificity." Biochem Biophys Res Commun 291(1): 91-100.

Frithz-Lindsten, E., Y. Du, R. Rosqvist and A. Forsberg (1997). "Intracellular targeting of exoenzyme $\mathrm{S}$ of Pseudomonas aeruginosa via type III-dependent translocation induces phagocytosis resistance, cytotoxicity and disruption of actin microfilaments." Mol Microbiol 25(6): 1125-1139.

Fu, H., J. Coburn and R. J. Collier (1993). "The eukaryotic host factor that activates exoenzyme S of Pseudomonas aeruginosa is a member of the 14-3-3 protein family." Proc Natl Acad Sci U S A 90(6): 2320-2324.

Ganesan, A. K., D. W. Frank, R. P. Misra, G. Schmidt and J. T. Barbieri (1998). "Pseudomonas aeruginosa exoenzyme S ADP-ribosylates Ras at multiple sites." J Biol Chem 273(13): 7332-7337.

Ganesan, A. K., T. S. Vincent, J. C. Olson and J. T. Barbieri (1999). "Pseudomonas aeruginosa exoenzyme $\mathrm{S}$ disrupts Ras-mediated signal transduction by inhibiting guanine nucleotide exchange factor-catalyzed nucleotide exchange." J Biol Chem 274(31): 21823-21829. 
Goehring, U. M., G. Schmidt, K. J. Pederson, K. Aktories and J. T. Barbieri (1999). "The N-terminal domain of Pseudomonas aeruginosa exoenzyme S is a GTPase-activating protein for Rho GTPases." J Biol Chem 274(51): 36369-36372.

Gordon, S. B., M. E. Molyneux, M. J. Boeree, S. Kanyanda, M. Chaponda, S. B. Squire and R. C. Read (2001). "Opsonic phagocytosis of Streptococcus pneumoniae by alveolar macrophages is not impaired in human immunodeficiency virus-infected Malawian adults." J Infect Dis 184(10): 1345-1349.

Graeff, R., Q. Liu, I. A. Kriksunov, M. Kotaka, N. Oppenheimer, Q. Hao and H. C. Lee (2009). "Mechanism of cyclizing NAD to cyclic ADP-ribose by ADP-ribosyl cyclase and CD38." J Biol Chem 284(40): 27629-27636.

Haag, F. and F. Koch-Nolte (1998). "Endogenous relatives of ADP-ribosylating bacterial toxins in mice and men: potential regulators of immune cell function." J Biol Regul Homeost Agents 12(3): 53-62.

Hassa, P. O., S. S. Haenni, M. Elser and M. O. Hottiger (2006). "Nuclear ADPribosylation reactions in mammalian cells: where are we today and where are we going?" Microbiol Mol Biol Rev 70(3): 789-829.

Hayaishi, O. and K. Ueda (1977). "Poly(ADP-ribose) and ADP-ribosylation of proteins." Annu Rev Biochem 46: 95-116.

Henriksson, M. L., C. Sundin, A. L. Jansson, A. Forsberg, R. H. Palmer and B. Hallberg (2002). "Exoenzyme S shows selective ADP-ribosylation and GTPase-activating protein (GAP) activities towards small GTPases in vivo." Biochem J 367(Pt 3): 617-628.

Hsia, J. A., J. Moss, E. L. Hewlett and M. Vaughan (1984). "ADP-ribosylation of adenylate cyclase by pertussis toxin. Effects on inhibitory agonist binding." J Biol Chem 259(2): 1086-1090.

Iglewski, B. H., J. Sadoff, M. J. Bjorn and E. S. Maxwell (1978). "Pseudomonas aeruginosa exoenzyme $\mathrm{S}$ : an adenosine diphosphate ribosyltransferase distinct from toxin A." Proc Natl Acad Sci U S A 75(7): 3211-3215.

Jansson, A. L., L. Yasmin, P. Warne, J. Downward, R. H. Palmer and B. Hallberg (2006). "Exoenzyme S of Pseudomonas aeruginosa is not able to induce apoptosis when cells express activated proteins, such as Ras or protein kinase B/Akt." Cell Microbiol 8(5): 815-822.

Knight, D. A. and J. T. Barbieri (1997). "Ecto-ADP-ribosyltransferase activity of Pseudomonas aeruginosa exoenzyme S." Infect Immun 65(8): 3304-3309.

Koch-Nolte, F. and F. Haag (1997). "Mono(ADP-ribosyl)transferases and related enzymes in animal tissues. Emerging gene families." Adv Exp Med Biol 419: 1-13. 
Krall, R., J. Sun, K. J. Pederson and J. T. Barbieri (2002). "In vivo rho GTPase-activating protein activity of Pseudomonas aeruginosa cytotoxin ExoS." Infect Immun 70(1): 360367.

Kreimeyer, A., P. Adamietz and H. Hilz (1985). "Alkylation-induced mono(ADPribosyl)-histones $\mathrm{H} 1$ and $\mathrm{H} 2 \mathrm{~B}$. Hydroxylamine-resistant linkage in hepatoma cells." Biol Chem Hoppe Seyler 366(6): 537-544.

Laing, S., M. Unger, F. Koch-Nolte and F. Haag (2011). "ADP-ribosylation of arginine." Amino Acids 41(2): 257-269.

Li, G., M. A. Barbieri, M. I. Colombo and P. D. Stahl (1994). "Structural features of the GTP-binding defective Rab5 mutants required for their inhibitory activity on endocytosis." J Biol Chem 269(20): 14631-14635.

Li, G. and P. D. Stahl (1993). "Structure-function relationship of the small GTPase rab5." J Biol Chem 268(32): 24475-24480.

Lodhi, I. J., R. E. Clift, G. M. Omann, J. F. Sweeney, K. K. McMahon and D. B. Hinshaw (2001). "Inhibition of mono-ADP-ribosyltransferase activity during the execution phase of apoptosis prevents apoptotic body formation." Arch Biochem Biophys 387(1): 66-77.

Lowery, R. G. and P. W. Ludden (1988). "Purification and properties of dinitrogenase reductase ADP-ribosyltransferase from the photosynthetic bacterium Rhodospirillum rubrum." J Biol Chem 263(32): 16714-16719.

Ludden, P. W. (1994). "Reversible ADP-ribosylation as a mechanism of enzyme regulation in procaryotes." Mol Cell Biochem 138(1-2): 123-129.

Luo, X. and W. L. Kraus (2012). "On PAR with PARP: cellular stress signaling through poly(ADP-ribose) and PARP-1." Genes Dev 26(5): 417-432.

Lupi, R., D. Corda and M. Di Girolamo (2000). "Endogenous ADP-ribosylation of the G protein beta subunit prevents the inhibition of type 1 adenylyl cyclase." J Biol Chem 275(13): 9418-9424.

Lupi, R., N. Dani, A. Dietrich, A. Marchegiani, S. Turacchio, C. P. Berrie, J. Moss, P. Gierschik, D. Corda and M. Di Girolamo (2002). "Endogenous mono-ADP-ribosylation of the free Gbetagamma prevents stimulation of phosphoinositide 3-kinase-gamma and phospholipase C-beta2 and is activated by G-protein-coupled receptors." Biochem J 367(Pt 3): 825-832.

McGuffie, E. M., D. W. Frank, T. S. Vincent and J. C. Olson (1998). "Modification of Ras in eukaryotic cells by Pseudomonas aeruginosa exoenzyme S." Infect Immun 66(6): 2607-2613. 
Moss, J. and M. Vaughan (1977). "Mechanism of action of choleragen. Evidence for ADP-ribosyltransferase activity with arginine as an acceptor." J Biol Chem 252(7): 24552457.

Moss, J. and M. Vaughan (1988). "ADP-ribosylation of guanyl nucleotide-binding regulatory proteins by bacterial toxins." Adv Enzymol Relat Areas Mol Biol 61: 303-379.

Mustafi, S., N. Rivero, J. C. Olson, P. D. Stahl and M. A. Barbieri (2013). "Regulation of Rab5 function in macrophages during phagocytosis of live Pseudomonas aeruginosa." Infect Immun.

Nikolova, L., K. Soman, J. C. Nichols, D. S. Daniel, B. F. Dickey and S. Hoffenberg (1998). "Conformationally variable Rab protein surface regions mapped by limited proteolysis and homology modelling." Biochem J 336 ( Pt 2): 461-469.

Olchowik, M. and M. Miaczynska (2009). "[Effectors of GTPase Rab5 in endocytosis and signal transduction]." Postepy Biochem 55(2): 171-180.

Paone, G., A. Wada, L. A. Stevens, A. Matin, T. Hirayama, R. L. Levine and J. Moss (2002). "ADP ribosylation of human neutrophil peptide-1 regulates its biological properties." Proc Natl Acad Sci U S A 99(12): 8231-8235.

Payne, J. F. and A. K. Bal (1976). "Cytological detection of poly (ADP-ribose) polymerase." Exp Cell Res 99(2): 428-432.

Pears, C. J., C. A. Couto, H. Y. Wang, C. Borer, R. Kiely and N. D. Lakin (2012). "The role of ADP-ribosylation in regulating DNA double-strand break repair." Cell Cycle 11(1): 48-56.

Sanford, J. C., Y. Pan and M. Wessling-Resnick (1995). "Properties of Rab5 N-terminal domain dictate prenylation of C-terminal cysteines." Mol Biol Cell 6(1): 71-85.

Scovassi, A. I. and G. G. Poirier (1999). "Poly(ADP-ribosylation) and apoptosis." Mol Cell Biochem 199(1-2): 125-137.

Stenmark, H., R. G. Parton, O. Steele-Mortimer, A. Lutcke, J. Gruenberg and M. Zerial (1994). "Inhibition of rab5 GTPase activity stimulates membrane fusion in endocytosis." EMBO J 13(6): 1287-1296.

Tezcan-Merdol, D., T. Nyman, U. Lindberg, F. Haag, F. Koch-Nolte and M. Rhen (2001). "Actin is ADP-ribosylated by the Salmonella enterica virulence-associated protein SpvB." Mol Microbiol 39(3): 606-619.

Ueda, K. and O. Hayaishi (1985). "ADP-ribosylation." Annu Rev Biochem 54: 73-100.

Vetter, I. R. and A. Wittinghofer (2001). "The guanine nucleotide-binding switch in three dimensions." Science 294(5545): 1299-1304. 
Williamson, K. C., L. A. Smith, J. Moss and M. Vaughan (1990). "Guanine nucleotidedependent ADP-ribosylation of soluble rho catalyzed by Clostridium botulinum C3 ADPribosyltransferase. Isolation and characterization of a newly recognized form of rhoA." Biol Chem 265(34): 20807-20812.

Zhang, J. (1997). "Use of biotinylated NAD to label and purify ADP-ribosylated proteins." Methods Enzymol 280: 255-265.

Zhang, Y., Q. Deng and J. T. Barbieri (2007). "Intracellular localization of type IIIdelivered Pseudomonas ExoS with endosome vesicles." J Biol Chem 282(17): 1302213032.

Zolkiewska, A. and J. Moss (1993). "Integrin alpha 7 as substrate for a glycosylphosphatidylinositol-anchored ADP-ribosyltransferase on the surface of skeletal muscle cells." J Biol Chem 268(34): 25273-25276. 


\section{Chapter 4}

The dynamics of Rin1 and Ras in the activation of Rab5 during phagocytosis of Pseudomonas aeruginosa 


\subsection{Abstract}

The Rab5 GTPase, a key regulator of early phagocytosis, was found to be modulated by $P$. aeruginosa T3S effector ExoS in J774-Eclone macrophages, resulting in impaired phagocytic activity. It was also observed that expression of Rab5-GEF Rin1, but not other GEFs (Rabex5 or Rap6) reversed this down regulation of Rab5 activity. In this current study, the molecular mechanism by which Rin1 rescues Rab5 activity has been investigated. On a time scale analysis of phagocytic indices at various time points of early phagocytic events, Rab5 activity was attenuated at a very early time point (2.5 min) of the process. Interestingly, upon over-expressing Rin1 in J774-macrophage cells, the Rab5 activity sustained for a prolonged time $(20 \mathrm{~min})$ reversing the negative effect from P. aeruginosa. Further investigations revealed Rin1 Vps9 domain together with Rin1 RA domain are required for optimal Rab5 activation. Since Rin1 RA domain is essential for Rin1 interaction with Ras as an effector protein, the dynamics of Rin1 and Ras interaction during live $P$. aeruginosa phagocytosis was investigated and Ras-Rin1 complex formation was confirmed during the period of early phagocytic processes. These observations highlight a novel mechanism of Rab5 activation and targeting to phagosome membrane during phagosytosis of live $P$. aeruginosa.

\subsection{Introduction}

Manipulation of Rab5 function is often adopted by intracellular bacteria during phagocytosis subversion (Brumell and Scidmore 2007; Alix, Mukherjee et al. 2011). Previously it has been shown that live $P$. aeruginosa strains selectively modulate Rab5 activity during early phagocytic events (Mustafi, Rivero et al. 2013). A key step in regulating phagocytosis is the activation of Rab5 GTPases by guanine nucleotide 
exchange factors (GEFs) of the Vps9 family (Carney, Davies et al. 2006) Ras effector Rin1 acts as a Rab5 GEF in endocytosis (Burd, Mustol et al. 1996; Horiuchi, Lippe et al. 1997; Tall, Barbieri et al. 2001) and promotes early endosome fusion (Galvis, Balmaceda et al. 2009). It was shown that Rin1 possesses putative Vps9 catalytic domains as well as functional Ras association (RA) domain (also known as Ras binding domain) (Colicelli, Nicolette et al. 1991; Ponting and Benjamin 1996; Han, Wong et al. 1997). The Rin1 protein contains a SH2 (Src homology 2) domain and a Proline rich domain on the amino-terminal region (henceforth termed Rin1 N) (Figure 21) whereas the Ras binding domain and Vps9 domain of Rin1 is localized in the carboxyl- terminal region (henceforth termed Rin1 C) (Figure 21) (Ponting and Benjamin 1996; Han, Wong et al. 1997). Han and others have shown that both in vitro and in vivo Rin1 binds directly to GTP bound Ras and that Rin1 binding requires Ras effector domain (Han and Colicelli 1995; Han, Wong et al. 1997). Later it was also shown that Rin1 directly competes with Ras effector Raf-1 for active Ras (Wang, Waldron et al. 2002).

The four small GTPases, H-Ras, N-Ras, and K-Ras of the Ras family function as molecular switches cycling between active GTP and inactive GDP bound forms. The Ras protein plays a pivotal role in the signal transduction of extra cellular ligands that activate a variety of cellular processes, including proliferation, differentiation, and metabolism (Shields, Pruitt et al. 2000). Several growth factors, hormones, chemokines and extracellular ligand-receptor complexes stimulate activation of Ras family (Ehrhardt, David et al. 2004). Activated GTP loaded Ras binds to several effector proteins like PI3K, Raf, Rin1 and activates other small GTPases such as Ral, Rap, Rab proteins (Ehrhardt, Ehrhardt et al. 2002). Rab5 activation during endocytosis has been linked to 
Ras signal transduction pathway (Li, D'Souza-Schorey et al. 1997; Barbieri, Kohn et al. 1998). Roberts and others showed that Ras activation resulted in Rab5 dependent stimulation of endocytosis and an increase in endosomal size (Roberts, Barbieri et al. 2000). Finally, Tall and others showed that Ras mediated activation of Rin1 stimulates Rab5 dependent endocytosis (Tall, Barbieri et al. 2001).

The Ras protein is a potent activator of actin polymerization during phagocytosis (Sasaki, Janetopoulos et al. 2007; Kortholt and van Haastert 2008; Clarke, Engel et al. 2010). Ras activation was also noticed at the phagocytic cup during Fc receptor mediated phagocytosis of opsonized particle in mouse macrophage cell line (Botelho, Harrison et al. 2009). LPS (Lipopolysaccharide) stimulates activation of Ras in primary macrophages (David, Cochrane et al. 2005). Pseudomonas aeruginosa stimulates up-regulation of mucin transcription in epithelial cells via activation of the Src-Ras-MEK1-ERK1pp90rsk-NF-kB pathway (Li, Feng et al. 1998). Alveolar macrophage, when first encounters $P$. aeruginosa during the process of lung infection, senses the bacterium and triggers an innate cellular response through a MyD88-dependent pathway. This MyD88 pathway involves downstream Ras signaling (Raoust, Balloy et al. 2009; Coste, Le Corf et al. 2010). Yet, there has been very few studies showing direct implication of Ras on $P$. aeruginosa phagocytosis, but there is ample evidence showing Ras as a target of $P$. aeruginosa virulence. In vivo, both oncogenic and normal Ras were ADP-ribosylated by P. aeruginosa type III effector ExoS (Vincent, Fraylick et al. 1999), whereas, constitutively active Ras protein protects cells from ExoS induced apoptosis (Jansson, Yasmin et al. 2006). 
It has been already established in a previous aim that over-expression of Rin1 FL protein in macrophage cell line was able to rescue phagocytic activity as well as active forms of Rab5 followed by live $P$. aeruginosa infection. In this chapter the molecular mechanism by which Rin1 rescues Rab5 activity during live $P$. aeruginosa phagocytosis has been explored. Over-expression of Rin1 FL protein in J774-Eclone cells prolonged the life span of GTP-bound Rab5 through the event of early phagocytosis, which was otherwise modulated by virulent factors of $P$. aeruginosa. Further investigations on Rin1 FL domains revealed the importance of Carboxyl-terminal domain (Rin1 C) which carries Rab5 activation domain and Ras association domain. The Rin1 C domain was responsible in increasing early phagocytic activity and killing of live P. aeruginosa. The Rin1 C domain was also shown to interact in vitro with GTP bound Ras as well as $P$. aeruginosa virulent factor ExoS mediated ADP-ribosylated GTP-Ras, although it showed reduced affinity for the modified GTP bound Ras. On the other hand, interaction between Rin1 C and Rab5 was significantly reduced when Rab5 was ADP-ribosylated by ExoS. Also, through Ras pull down, Rin1 was successfully precipitated from J774-Eclone cells carrying out phagocytosis of live $P$. aeruginosa. Together, these observations illustrate the importance of Ras-Rin1 pathway in re-establishing Rab5 activity during phagocytosis of $P$. aeruginosa. 


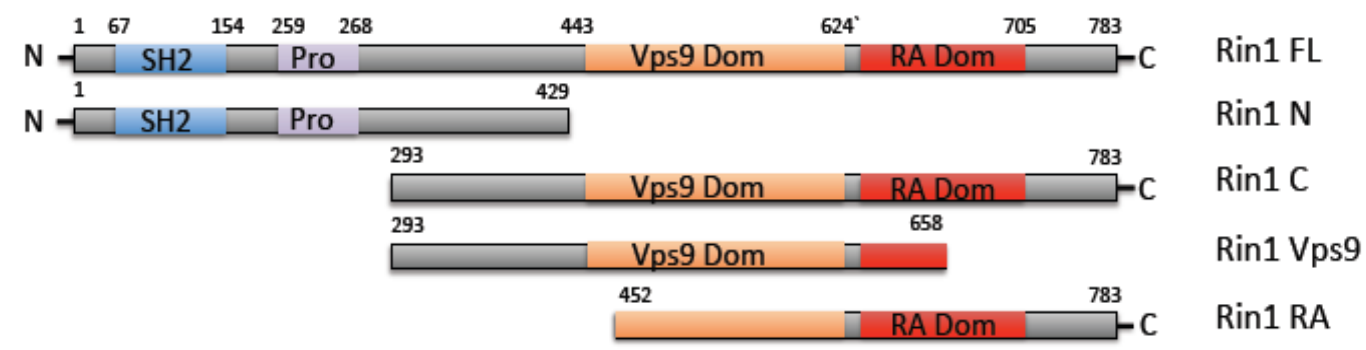

Figure 21 Schematic representation of different Rin1 protein constructs.

From top, Rin1 FL flaunting intact protein sequence with 783 amino acids showing SH2, Proline rich, Vps9 and RA domain. Rin1 N, the amino terminal region of the Rin1 protein, 429 amino acids long, containing SH2 and Proline rich domain. Rin1 C, the carboxyl terminal region of Rin1, starting at 293 amino acid and ending at 783 amino acid, contains Vps9 and RA domains. Rin1 Vps9 containing on Vps9 domain of Rin1 protein, encoding amino acid from position 293-658 and Rin1 RA, encoding amino acids 452-783 and containing RA domain of Rin1 protein.

\subsection{Materials and Methods}

\subsubsection{Materials}

All chemicals and reagents were purchased from Sigma-Aldrich (Saint Louis, MO), unless otherwise indicated. Primary and secondary antibodies used in immunoblotting were purchased from Cell Signaling Technology Inc. (Danvers, MA). Culture supplies were purchased from Invitrogen life technologies (Carlsbad, CA).

\subsubsection{Cell culture}

The J774 E-clone cells were kindly provided by Dr. Philip Stahl (Washington University Medical School, St Louis, MO) and were maintained as described previously 
(Mustafi, Rivero et al. 2013). Briefly, they were maintained under a 5\% $\mathrm{CO}_{2}$ atmosphere in Dubelco's minimum essential medium (DMEM), supplemented with $10 \%$ heatinactivated fetal calf serum (FCS), $2 \mathrm{mM} \mathrm{L}$-glutamine, $100 \mathrm{Units} / \mathrm{mL}$ penicillin, 100 $\mu \mathrm{g} / \mathrm{mL}$ of streptomycin. J774-Eclone cells were used for all P. aeruginosa phagocytosis studies. Platinum-E retroviral packaging cell line (Plat-E cells) were purchased from Cell Biolabs, Inc. (San Diego, CA) and maintained in DMEM, 10\% FCS, $1 \mu \mathrm{g} / \mathrm{mL}$ puromycin, $10 \mu \mathrm{g} / \mathrm{mL}$ blasticidin, 100 Units/ $\mathrm{mL}$ penicillin, $100 \mathrm{mg} / \mathrm{mL}$ of streptomycin.

\subsubsection{Bacteria strains}

Pseudomonas aeruginosa strains PAO1 (derivative of original Australian isolate PAO) were provided by Dara Frank (Medical College of Wisconsin, Milwaukee, WI). PAO1 strain expressing GFP protein (a kind gift from Dr. Matthew Parsek, Washington University, St. Louis, MO) were used in microscopic studies. Bacteria were grown at $37^{\circ} \mathrm{C}$ in Lauria Broth with appropriate antibiotics. Prior to the phagocytosis assay, bacteria were grown to late-log phase and diluted to a concentration of $10^{7} \mathrm{cfu} / \mathrm{mL}$, and added to cells at indicated MOIs.

\subsubsection{Construction of recombinant $\mathrm{pMX}$-puro retroviruses and cell lines}

The cDNAs of Rin1 FL, Rin1 N (amino acids 1 to 293), Rin1 C (amino acids 293 to 783), Rin1 Vps9 (amino acids 293 to 658) and Rin1 RA (amino acids 452 to 783 ) were subcloned into the pMX-puro vector as previously described by Barbieri and others (Barbieri, Kong et al. 2003). The cDNAs were used in the FUGENE6-mediated transfection of $90 \%$ confluent Plate-E cell monolayers. Cells were maintained at $37^{\circ} \mathrm{C}$, and the medium containing released virus was harvested 48 hour after transfection. Viral stocks were aliquoted and frozen at $-80^{\circ} \mathrm{C}$ until use. Cell lines were generated by 
infecting J774E-clone cells with retrovirus encoding PMX-puro vector only, Rin1 FL, Rin1 N, Rin1 C, essentially as previously described (Barbieri, Kong et al. 2003).

\subsubsection{Phagocytosis assay}

Pseudomonas aeruginosa strains were cultured to late-log phase, washed with phosphate buffered saline (PBS, $\mathrm{pH}$ 7.3) and then with $\mathrm{NaHCO}, \mathrm{pH} 9$, three times, respectively. After washing, Alexa fluor-594 (Invitrogen, Carlsbad, CA) was used to label live or heat-inactivated bacteria for $2 \mathrm{hrs}$ at room temperature while protected from light. J774-Eclone cells $\left(10^{5}\right.$ cells $\left./ \mathrm{mL}\right)$ were plated on coverslips in 6-well plates and incubated overnight. Cells were washed once with PBS and then twice with Hanks balanced salt solution-2\% BSA. Bacteria were added at a ratio of 200:1 and incubated for 30 minutes at $4^{\circ} \mathrm{C}$. To initiate bacterial internalization, plates were placed in a $37^{\circ} \mathrm{C}$ water bath for 1 minutes, 2.5 minutes, 5 minutes, 10 minutes, 15 minutes, 20 minutes, 30 minutes, 45 minutes and 60 minutes respectively. After this time, cells were placed on ice and washed three times with PBS, and then fixed for 20 minutes at room temperature using 3.7\% paraformaldehyde. After fixation, cells were washed three times with PBS, incubated with $1 \%$ Triton X-100 at room temperature for 15 minutes, and incubated with 4',6-diamidino-2-phenylindole (Roche Applied Science, Indianapolis, IN) to stain the nucleus. Coverslips were removed from the wells, washed and mounted with Mowiol fluorescence mounting medium. The number of bacteria per cell was enumerated at 100X magnification using a phase-contrast inverted fluorescent microscope. Two hundred cells were counted per slide, and each experiment was repeated three times. The phagocytic index refers to the number of bacteria inside each cell. 


\subsubsection{Bacterial survival assay}

An antibiotic protection assay, described by Goldova et al. , (Angus, Evans et al. 2010) was used to analyze bacterial survival within macrophages. For this assay, following the indicated $30 \mathrm{~min}$ time of phagocytosis, cells were washed 3 times with PBS, and extracellular bacteria were killed by incubating cells with growth medium containing Amikacin $(400 \mu \mathrm{g} / \mathrm{mL})$. After washing, cells were incubated at $37^{\circ} \mathrm{C}$ for an additional 30 minutesto examine bacterial survival within macrophages. Finally, cells were washed with PBS, lysed with $0.5 \%$ TritonX-100, and lysates were plated on LBagar, incubated overnight and bacterial colonies were enumerated.

\subsubsection{Recombinant protein purification}

The Rab5:S34N (Mustafi, Rivero et al. 2013) construct was cloned into protein expression vector pET28a between Hind III and BamH1. The H- Ras V12 construct in pET28a vector (Tall, Barbieri et al. 2001), Glutathione S-transferase (Botelho, Harrison et al. 2009)-fused 14-3-3 protein (pGEX4t-14-3-3) and GST-Rin1 C (Barbieri, Kong et al. 2003) were received as a kind gift from Dr. Phil Stahl (Washington University, St. Louis, MO). The ExoS-pET16b construct was generously provided by Dr. Michele Vidal (Université Montpellier, France). The H-Rab5:S34N, H-Ras V12 and ExoS carrying Histidine tag (His tag) were expressed in E.coli and purified following previously established protocol (Knight, Finck-Barbancon et al. 1995). The GST-Rin1 C and GST14-3-3 proteins were purified as previously described by Barbieri and others (Barbieri, Li et al. 1994). 


\subsubsection{In vitro ADP-ribosylation assay}

ADP-ribosylation assay for His-Rab5:S34N was carried out essentially as previously described with few modifications (Fraylick, 2002)(Barbieri, Tall et al. 2001). Briefly a $50 \mu 1$ reaction mixture of His Rab5:S34N protein $(1 \mu \mathrm{g})$, His-ExoS $(0.8 \mu \mathrm{M}), \mathrm{GST}-14-3-3$ $(0.8 \mu \mathrm{M}), 0.2 \mathrm{M} \mathrm{Na}$ acetate $(\mathrm{pH} 6.0), 1 \mathrm{mM}$ Magnesium chloride and $10 \mathrm{mM}$ Biotinylated NAD (8-([N-biotinyl(6-aminohexyl)]amino)NAD) was incubated at $25^{\circ} \mathrm{C}$ for 1 hour. For SDS-PAGE analysis, reaction was stopped by adding $4 \mathrm{x}$ Leammli sample buffer and boiled for 5 minutes at $95^{\circ} \mathrm{C}$. ADP-ribosylation reaction for His-H-Ras ${ }^{\mathrm{V} 12}$ were run at $25^{\circ} \mathrm{C}$ for 1 hour in a $50 \mu \mathrm{l}$ reaction mixture of $0.2 \mathrm{M}$ Tris-acetate, $\mathrm{pH} 6.0,1 \mathrm{mM} \mathrm{MgCl} 2$, 10mM Biotinylated NAD, 0.2mM 14-3-3, 0.5 mM His-H-Ras:G12V and 0.2 mM ExoS as previously described by Vincent and others (Vincent, Fraylick et al. 1999). For SDSPAGE analysis, reaction was stopped by adding 4x Leammli sample buffer and boiled for 5 minutes at $95^{\circ} \mathrm{C}$. Electrophoretic mobility shift of ADP-ribosylated Rab5 and Ras was detected by immunoblotting (IB) using rabbit anti-Rab5 antibody or rabbit anti Ras antibody (Cell signaling, Denver, MA).

\subsubsection{In vitro pull down assays}

One hundred $\mu \mathrm{l}$ glutathione beads containing 3mM of GST-Rin1 C were washed in $10 \mathrm{mM}$ Tris- $\mathrm{HCl}(\mathrm{pH} 7.6), 20 \mathrm{mM} \mathrm{NaCl}$ and stored at $4{ }^{\circ} \mathrm{C}$. Three $\mathrm{mM}$ of ADPribosylated Rab5:S34N or H-Ras:G12V (as described earlier) were added to immobilized GST-Rin1 C for 30 minutesat $4{ }^{\circ} \mathrm{C}$ in a total volume of $75 \mathrm{ml}$. Samples were centrifuged and pelleted material was washed with $100 \mathrm{ml}$ of $10 \mathrm{mM}$ Tris- $\mathrm{HCl}(\mathrm{pH} 7.6), 20 \mathrm{mM}$ $\mathrm{NaCl}$ and was subjected to SDS-PAGE. Proteins were transferred to nitrocellulose and analyzed by immunoblotting (IB) using appropriate antibodies. 


\subsubsection{Ras-Rin1 Co-Immuno Precipitations in Vivo}

Confluent cultures of J774 Eclone cells, control or over-expressing Rin1 FL, in $35 \mathrm{~mm}$ dishes were exposed to bacteria and followed by phagocytosis as described above. Ras and Rin1 FL co-immunoprecipitation was performed essentially as described previously (Vincent, Fraylick et al. 1999). Cells were lysed in $1 \mathrm{ml}$ of ice-cold Co-IP buffer (30mM HEPES, pH7.5, 1\%Triton X-100, 10mM sodium chloride, 10\% glycerol, $1 \mathrm{mM}$ EGTA, $25 \mathrm{mM}$ sodium fluoride, $1 \mathrm{mM}$ sodium orthovanadate, $10 \mathrm{mM} \beta$ glycerophosphate, $10 \mathrm{mM}$ benzamidine, $0.1 \mathrm{mM}$ phenylmethylsulphonyl fluoride, 10 $\mu \mathrm{g} / \mathrm{mL}$ aprotinin, $10 \mu \mathrm{g} / \mathrm{mL}$ leupeptin and $10 \mu \mathrm{g} / \mathrm{mL}$ trypsin inhibitor). The Ras proteins were precipitated for $2 \mathrm{~h}$ at $4^{\circ} \mathrm{C}$ with $1.5 \mathrm{mg}$ of rabbit monoclonal anti Pan-Ras antibody (Calbiochem, CA) and $10 \mathrm{ml}$ of a $50 \%$ slurry of Protein A conjugated to sepharose beads. Immuno-complexes were washed four times in Co-IP wash buffer (50mM HEPES, $\mathrm{pH}$ 7.5, $100 \mathrm{mM}$ sodium chloride, $0.1 \%$ Triton $\mathrm{X}-100,10 \%$ glycerol and $20 \mathrm{mM}$ sodium chloride) and subjected to immunoblotting. Ras was immunoblotted with rabbit polyclonal antibody (Cell signaling, Denver, MA).

\subsubsection{Rab5 activation assay}

The activation of Rab5 protein was detected by configuration specific monoclonal antibody based Rab5 activation kit (New East Biosciences, Malvern, PA). Briefly, J774Eclone cells were seeded at $1 \times 10^{8}$ cells $/ \mathrm{ml}$ and phagocytosis was activated as described earlier. Cells were then lysed with lysis buffer (New East Biosciences, Malvern, PA) according to vendor's instruction and lysates were incubated with anti-Rab5 GTP monoclonal antibody and protein $\mathrm{A} / \mathrm{G}$ agarose bead slurry according to the instructions and incubated at $4^{\circ} \mathrm{C}$ for $1 \mathrm{~h}$. As a control, equal amount of lysate protein was incubated 
only with protein A/G agarose beads. Finally, beads were washed with lysis buffer and resuspended in sample buffer for immunoblotting using rabbit polyclonal anti Rab5 antibody (cell signaling, Denver MA). Positive control for this experiment was GTP $\gamma \mathrm{S}$ treated cell lysates and negative control was GDP added cell lysates prepared as per vendor's instruction.

\subsubsection{Ras activation assay}

The Ras activation kit (Cell signaling, San diego, CA) was used to assay for active Ras. Briefly, J774-Eclone cells were seeded at $1 \times 10^{8}$ cells $/ \mathrm{ml}$ and phagocytosis was activated as described before. Cells were then lysed with lysis buffer (Cell signaling, Denver MA) according to vendor's instruction. One ml of lysate was incubated with Raf1 $\mathrm{RBD}$ agarose bead (Cell signaling, Denver MA) slurry for $1 \mathrm{~h}$ at $4^{\circ} \mathrm{C}$ and then beads were pelleted and washed before subjecting to immunoblot using Rabbit polyclonal anti Ras antibody (Cell signaling, Denver MA). Positive control for this experiment was GTP $\gamma \mathrm{S}$ treated cell lysates and negative control was GDP added cell lysates prepared as per vendor's instruction.

\subsubsection{Cell lysis and immunoblotting}

For immunoblot analysis, J774-Eclone cells were washed twice with PBS and then lysed with RIPA cell lysis buffer (50 mM Tris-HCl, $150 \mathrm{mM} \mathrm{NaCl}, 0.1 \%$ SDS, $0.5 \%$ sodium deoxycholate, $1 \%$ NP40) in the presence of protease and phosphatase inhibitors. Lysates were collected with cell scrapers and cleared by centrifugation. Prior to SDSPAGE, cell lysates were resuspended in SDS sample buffer (60 mM Tris-HCl, 1\% (w/v) SDS, $10 \%$ glycerol, $0.05 \% \quad(\mathrm{w} / \mathrm{v})$ bromophenol blue, $\mathrm{pH} \quad 6.8$, with $2 \% \quad \beta$ mercaptoethanol). Samples were subjected to SDS-PAGE and transferred to 
nitrocellulose membranes for immunoblotting. Nitrocellulose membranes were incubated with blocking solution (TBS containing $0.1 \%$ Tween 20 and 5\% BSA) and were probed with the indicated antibodies

\subsubsection{Image quantification}

The NIH Image J64 was used to quantify Western blots after images were scanned at a grayscale amplification of 600 dpi. Digital images of the Western blot from cell lines were captured and loaded into ImageJ64, and-Rab5 specific bands, along with $\alpha$-tubulin bands, were assessed in each sample using the Analyze $\rightarrow$ Gels function, which allows for background correction. The ratio of Rab5 signal to $\alpha$-tubulin was calculated for each sample and served as an index of Rab5 expression. The index of expression for other proteins examined in this study was derived in a similar manner.

\subsubsection{Statistical analysis}

All samples in this study were analyzed in duplicate and each experiment was repeated three times. Values represent the mean \pm SEM of three independent experiments. To compare two groups, student's t test was used and $P<0.05$ was considered as statistically significant.

\subsection{Results}

\subsubsection{Rab5 activation is interrupted shortly after initiation of live Pseudomonas aeruginosa phagocytosis.}

Previous studies suggest that the time points of early phagocytic events vary between 5 to 20 minutespost activation (Allen, Yang et al. 2002; Arora, Chan et al. 2005). Evidence of Rab5 activation during phagocytosis is consistent with the data and has been shown to peak between 5 to 20 minutesin several phagocytosis experiments (Horiuchi, 
Lippe et al. 1997; Roberts, Barbieri et al. 2000). It has been shown that live $P$. aeruginosa blocks Rab5 activity at 15 minutespost infection (Mustafi, Rivero et al. 2013). In this present study, the activity of Rab5 was examined for the entire time span of early phagocytosis. For this experiment, J774-Eclone cells expressing pMX vector (control cells) were incubated with live or heat inactivated $P$. aeruginosa and after initiation, phagocytosis was continued up to 1 minute, 2.5 minutes, 5 minutes, 10 minutes, 15 minutes, 20 minutes, 30 minutes and 60 minutes, respectively. Cell lysate from each phagocytic time points were analyzed for their total content of Rab5 using immunoblot technique (Figure $22 \mathrm{~A}$ and B). Rab5-GTP bound state was also analyzed using Rab5-GTP pull down assay with anti Rab5-GTP monoclonal antibody and agarose beads encoded with protein A/G. During phagocytosis of heat inactivated P. aeruginosa, GTP bound active Rab5 was detected between 1 minute and 20 minutes of phagocytosis (Figure 22A). Macrophage cells undergoing phagocytosis of live P. aeruginosa, showed curtailed life span of GTP bound Rab5. As shown in Figure 22B, GTP bound Rab5 was detected at 1 minute and 2.5 minute of phagocytosis, but slowly faded from 5 minutes onwards. At 5 minute time point, Rab5-GTP diminishes almost about $98 \pm 5 \%$ in macrophages carrying live $P$. aeruginosa from that of macrophages carrying heat inactivated $P$. aeruginosa. These results are consistent with the observations in the previous aim, confirming the inhibition of Rab5 activity during phagocytosis of live $P$. aeruginosa and also demonstrating the effective time span of Rab5 activity during the phagocytosis of both heat inactivated and live $P$. aeruginosa. 


\subsubsection{Rin1: FL over-expression in macrophage cells help to retain Rab5-active form at the early phagocytic time points during Pseudomonas aeruginosa invasion.}

The Rab5-GEF, Rin1 that partially reversed negative effect of live $P$. aeruginosa on Rab5 activity (Mustafi, Rivero et al. 2013) was introduced to this system to investigate the mechanism of such effect. At first, Rab5 activity was measured for the entire phase of early phagocytosis in J774-Eclone cells in presence of over-expressed Rin1: FL protein. To determine whether Rin1 activity influenced the sustenance of Rab5-GTP bound form, J774-Eclone cells expressing Rin1 FL were incubated with both live and heat inactivated P. aeruginosa from 1 to 60 minutes as previously stated and analyzed for Rab5-GTP using immunoblot technique with monoclonal anti-Rab5-GTP antibody. As described in Figure $23 \mathrm{~B}$, Rab5-GTP was detected from 1 to 20 minutes during live P. aeruginosa phagocytosis. Similar to previous observations, Rab5 activity did not alter in macrophage cells carrying heat inactivated $P$. aeruginosa in control experiment (Figure 23A). Although Rab5-GTP appeared to have sustained longer in Rin1 FL over-expressing macrophages carrying live bacteria, there was at least $40 \pm 5 \%$ decline of the activity by 15 minute time point. Together these results suggest that in presence of Rin1 FL, Rab5-GTP bound form remains intact and sustains negative effects of live $P$. aeruginosa effectors corroborating with previous results (Mustafi, Rivero et al. 2013).

4.4.3 Selective role of Rin1 domains in Rab5 activation during internalization of live Pseudomonas aeruginosa.

The substantial role of Rin1 in reinstating Rab5 activity is eminent from previous results. In order to investigate the mechanism by which Rin1 influence Rab5 to overcome the interference in its activity during live $P$. aeruginosa phagocytosis, Rin1 domains were 
introduced separately into the macrophage cells. The amino terminal region of Rin1, Rin1 N contains SH2 domain followed by a Proline rich region. The carboxyl terminal region of Rin1, Rin1 C contains Vps9 domain and RA domain (Figure 21). Macrophage cells over expressing Rin1 FL, Rin1 N or Rin1 C were incubated with live $P$. aeruginosa and its internalization was assayed at different time points over a period of 30 minutes. Expression of Rin1 FL and Rin1 C proteins overall increased the internalization of live $P$. aeruginosa in comparison to control cells and cells expressing Rin1 N (Figure 24A). Further, Rin1:Vps9 and Rin1:RA domains were separately over-expressed in the same macrophage cell system and phagocytic index for live P. aeruginosa was analyzed. Data indicated no significant impact of each of neither these domains on phagocytic index assuring neither Vps9 nor RA domain solely interplay in Rab5 activation (Figure 24B). Followed by internalization, cells were then analyzed for in vivo bacterial survival. Cells were incubated in $P$. aeruginosa free media for an additional 30 minutes post phagocytosis. Finally cells were washed with ice cold PBS and treated with amikacin $\left(400 \mu \mathrm{g} / \mathrm{mL}\right.$ ) for 2 hours at $4^{\circ} \mathrm{C}$ to kill any bacteria attached to the cells. Afterwards, cells were lysed with $0.5 \%$ triton $\mathrm{X}$ and plated on LB-agar for internalized bacterial viability. Previous experiment provided the total number of bacteria internalized which supported as a control. Results indicated that unlike internalization, bacterial survival rate sharply decreased in cells over-expressing Rin1 FL and Rin1 C in comparison to control cells and cells over-expressing Rin1 N (Figure 24C).

\subsubsection{Ras is active in early phase of Pseudomonas aeruginosa phagocytosis.}

Ras association domain in Rin1 C region has been found to interact with Ras in its active state (Wang, Waldron et al. 2002). Observing the role of Rin1 C in P. aeruginosa 
phagocytosis, it was tempting to examine the activity of Ras during the process as well. GTP- bound endogenous Ras form were assayed in J774-macrophage cells carrying phagocytosis of live or heat inactivated $P$. aeruginosa. Following the phagocytosis, cells were washed with PBS and lysed with lysis buffer and active Ras was probed with Raf1RBD pull down assay as described in Material Methods. As shown in Figure 25, GTP bound Ras was detected between 1-15 minutes and peaked during 2.5 - 10 minutes period of early phagocytosis. There were no significant differences in endogenous Ras activation during the process of phagocytosis in both live (Figure 25B) or heat inactivated (Figure 25A) $P$. aeruginosa. The result was also confirmed by measuring phosphorylated ERK from the cell lysates with the help of immunoblot technique.

\subsubsection{Rin1 FL interacts with Ras in Pseudomonas aeruginosa phagocytosis.}

Previous results indicate that Ras activity remains unaltered during early phagocytic events of $P$. aeruginosa. To determine whether Rin1 binds to Ras in response to phagocytic signals during live $P$. aeruginosa invasion a pull down protocol was employed. Followed by phagocytosis of live $P$. aeruginosa for 1 minute, 2.5 minutes, 5 minutes, 10 minutes, 15 minutes and 20 minutes, Rin1 FL over-expressing macrophage cells were lysed and incubated with mouse monoclonal Pan-Ras antibody and agarose beads encoded with protein A. Cellular Ras bound to Pan-Ras antibody was then precipitated with protein A and probed with rabbit polyclonal anti-Ras antibody through immunoblotting. The Rin1 FL protein was also probed with Rin1 antibody in the same samples. As shown in Figure 26, Rin1 FL was precipitated with pulled down Ras at several time points such as 2.5 minutes, 5 minutes, 10 minutes, 15 minutes, 20 minutes, 
etc. The maximum precipitation takes place between 5-10 minutes suggesting Rin $1 \mathrm{FL}$ interacts with Ras protein during the early phases of phagocytosis of live P. aeruginosa.

\subsubsection{Carboxyl terminal domain of Rin1 protein selectively interacts with modified and unmodified Ras and Rab5 protein in vitro.}

The affinity and specificity of Rin1 for modified Ras and Rab5 were examined using in vitro binding assays. Rin1 protein, specifically Rin1 $\mathrm{C}$ has maximum affinity for GTP bound Ras (a constitutively active Ras mutant: Ras:G12V) and GDP bound Rab5 (Rab5:S34N). Here, the affinity of Rin1 C toward modified and unmodified Rab5:S34N and Ras:G12V was examined. Rab5:S34N and Ras:G12V were ADP-ribosylated in vitro by ExoS as described in material methods (Figure $27 \mathrm{~A}$ and C). Afterwards both normal and ADP-ribosylated Rab5:S34N and Ras:G12V were incubated with Rin1 C immobilized in GST agarose beads. The GST beads were then precipitated and subjected to immunoblot to probe for Rin1 C bound Rab5:S34N and Rin1 C- bound Ras:G12V. As expected, both Rab5:S34N and Ras:G12V show 100\% affinity for Rin1 C (Figure 27 B and D). The ADP-ribosylated Rab5:S34N shows little or no affinity towards Rin1 C, whereas ADP-ribosylated Ras shows $40 \pm 6 \%$ affinity towards Rin1 C. This is an indication that in vitro Rin1 C is sensitive toward any modification of Rab5:S34N but not so for Ras:G12V.

\subsection{Discussion}

The Rin1 protein, a Rab5 GEF, was found to regulate membrane trafficking and endosome fusion in co-ordination with Rab5 (Gorvel, Chavrier et al. 1991; Bucci, Parton et al. 1992; Tall, Barbieri et al. 2001; Barbieri, Kong et al. 2003; Barbieri, Fernandez-Pol et al. 2004). Rin1 is also a Ras effector protein that regulates endocytosis and signaling of 
receptor tyrosine kinases (Barbieri, Fernandez-Pol et al. 2004). This current study demonstrates the role of Rin1 as a Rab5-GEF in phagocytosis of live P. aeruginosa. During phagocytosis of live $P$. aeruginosa, Rab5 activity in J774-Eclone macrophage cells was diminished at a very early point after initiation (2.5 minutes). Once Rin1 FL protein was over-expressed in macrophage cells, the activity sustained for a longer time which is relevant with early phagocytic time points. In order to investigate the possible role of Rin1 Vps9 domain for boosting Rab5 activity, Rin1 N and Rin1 C were separately over-expressed in macrophage cells. Rin1 C seemed to be as effective as Rin1 FL protein to revive phagocytic activity in macrophage cells during live $P$. aeruginosa invasion. Since Rin1 C encodes RA domain, the possible role of Ras in live P. aeruginosa invasion was also investigated. Interestingly, it was noticed that live $P$. aeruginosa did not alter Ras activity in early phagocytic time points. When Rin1 over expressed macrophage cells were analyzed for Ras-Rin1 interaction, Rin1 appeared to be bound with Ras during early phagocytic time points of live $P$. aeruginosa invasion. In vitro studies revealed disrupted interaction between Rin1 $\mathrm{C}$ and ADP-ribosylated Rab5. But ADP-ribosylation modification in Ras does not completely disrupt its interaction with Rin1 C. From in vitro observations, it could be suggested that the intrinsic Rab5 activation cycle by overexpressed Rin1 could be interrupted upon large accumulation of live $P$. aeruginosa at the site of activation. Together, these data suggest a possible role of Rin1- Ras coordinated activation of Rab5 in rescuing phagocytic activity during live P. aeruginosa invasion.

Endogenous Rab5 activity is modulated by $P$. aeruginosa T3S effector ExoS at 15 minutes into phagocytosis resulting in diminished phagocytic index (Mustafi, Rivero et al. 2013). In this study the activity log of endogenous Rab5 was documented at multiple 
time points. Internalization of heat inactivated $P$. aeruginosa did not alter Rab5 activity. As shown in Figure 22 A, Rab5-GTP was detected between 1 and 20 minutes from phagocytosis start point. Immunoblot analysis of Rab5-GTP indicates activity peaked around 15-20 minutes where as, a bleak life span of Rab5-GTP was recorded in macrophage cells with live $P$. aeruginosa. In this case, GTP bound Rab5 was detected from 1-2.5 minutes, and was significantly diminished by $98 \pm 5 \%$ around 5 minutes of phagocytosis when compared to Rab5-GTP from the heat inactivated $P$. aeruginosa phagocytosis.

Some earlier studies have shown that Rin1 depletion resulted in defective endosome fusion which was then reversed by newly introduced Rin 1 in orchestration with Rab5 and Rab5 effector EEA1 (Galvis, Giambini et al. 2009). Also, Rin1 over-expression in J774macrophage was able to rescue Rab5 activity and enhance phagocytic index (Mustafi, Rivero et al. 20013). In this study, upon over expressing Rin1 FL with the aid of pMXpuro retroviral system in J774-Eclone cells, Rab5 activity log was altered during live $P$. aeruginosa invasion. Rab5-GTP was significantly elevated and the duration of the activity prolonged up to 20 minutes (Figure 23B). Under a similar situation Rab5 activity appeared between 1-30 minutes in macrophages engulfing heat inactivated $P$. aeruginosa (Figure 23A). This observation could be the result of an intrinsic Rab5 guanine exchange cycle generated by Rin1 over-expression in macrophage cells. In both cases of live and heat inactivated $P$. aeruginosa phagocytosis, the presence of Rab5-GTP beyond the usual duration of early phagocytosis, could be attributed to Rin 1 mediated feedback cycle of Rab5 activity. However, as shown in Figure 23A, Rab5-GTP appeared strong at 30 minute of heat inactivated $P$. aeruginosa phagocytosis, about $80 \pm 4 \%$ more than that in 
macrophages with live $P$. aeruginosa. Although the Rab5 modification by live $P$. aeruginosa T3S effectors seems to be masked by Rin1 GEF activity, there could be a possible interference in the equilibrium between modified and unmodified Rab5.

To further analyze the possible molecular mechanism several Rin1 domains were separately expressed in J774-Eclone cells. The SH2 domain and a Proline rich domain containing Rin1 N and Vps9 and RA domain containing Rin1 C were observed for their role in phagocytosis. Both Rin1 FL and Rin1 C up regulated phagocytic activity by two fold compared to J774-Eclone control cells carrying only the pMX vector. When survival of phagocytized bacteria was estimated following phagocytosis, Rin1 FL over-expressing macrophage cells showed $30 \pm 6 \%$ survival rate and Rin $1 \mathrm{C}$ over expressing cells showed $40 \pm 5 \%$ less survival for internalized live bacteria (Figure 24B). However, control cells and Rin1 $\mathrm{N}$ over expressing cells showed limited phagocytic activity for live $P$. aeruginosa and did not alter the survival of the internalized bacteria. With these results, the importance of Rin1 C could be attributed to boost Rab5 activity. Further, when separate domains such as Rin1 Vps9 and Rin1 RA over-expressing macrophage were subjected to phagocytosis, they did not affect the phagocytic index and were very similar to control cells. Taken together, results from Figure 24 strongly indicate that Rin1 Vps9 domain in conjunction with Rin1 RA domain potentiates Rab5 activity during phagocytosis of live $P$. aeruginosa. Another important fact was observed that even removal of the $\mathrm{SH} 2$ and proline-rich domains of Rin1causes no observable defect in the ability of Rin1 to stimulate phagocytic activity.

Previously in a study by Roberts and others it has been suggested that the mechanism of Ras induced Rab5 activation and accumulation of Rab5 at the site of vesicle formation 
and vesicle fusion is essentially similar in both endocytosis and phagocytosis (Roberts, Barbieri et al. 2000). A functional correlation has been established between Vps9 domain and RA domain found in Rin1 in a novel Ras mediated endocytosis pathway, where essentially GTP- bound active Ras interacts with Rin1 through RA domain which potentiates enzymatic activity in Rin1 Vps9 domain (Tall, Barbieri et al. 2001). Rin1 has higher affinity for activated Ras when compared to other potent Ras effector Rafl (Wang, Waldron et al. 2002). This is consistent with the results from this study indicating a dual role of Ras and Rin1 in rescuing Rab5 activity during phagocytosis of live P. aeruginosa. $P$. aeruginosa mediated induction of Ras-MAPK pathway has been essentially found in epithelial cells (Li, Feng et al. 1998). Between the early phagocytic time points, Ras activity was observed to peak within a short span of 2.5 minutes and 10 minutes during both live or heat inactivated $P$. aeruginosa engulfment. Correlating the Ras activity, pERK was also observed at those time points (Figure 25). Considering the fact that live $P$. aeruginosa T3S effectors modify both Ras and Rab5 (Ganesan, Vincent et al. 1999; Barbieri, Tall et al. 2001), in vivo interaction between Rin1 and Ras protein was analyzed in macrophages internalizing live $P$. aeruginosa. Immuno-precipitation of endogenous Ras illustrated that Rin1-Ras interaction occurs between 2.5 and 20 minutes of phagocytosis and by 30 minutes, the interaction dissipated by $95 \pm 7 \%$ (Figure, 26). Given the strong correlation of Ras and Rab5 activity tethered together by Rin1, it was important to analyze the same between ADP-ribosylated Ras and ADP-ribosylated Rab5. ADP-ribosylation of Ras disrupts Ras-Raf1 signaling in in vivo (Zhang, 2007; Ganesan, 1999). In vitro, ADP-ribosylated Ras:G12V showed 55 $\pm 6 \%$ reduced affinity toward Rin1 C but ADP-ribosylated Rab5 interaction with Rin1 C was completely disrupted (Figure 
27). Ras protein in host cell is a major target for $P$. aeruginosa T3S ExoS but it failed to down regulate constitutively active Ras (Jansson, Yasmin et al. 2006). Also, it has been found that ExoS mediated Ras modification disrupts Ras-GEF interaction rather than Ras-effector interaction (Ganesan, Vincent et al. 1999). Similarly, ExoS failed to modify constitutively active form of Rab5 (Barbieri, Tall et al. 2001; Mustafi, Rivero et al. 2013). From in vitro studies in this work, it could be conjectured that Rab5 modification disrupts Rin1 interaction but partially disrupts Ras-Rin1 interaction.

Overall in this study, a novel pathway has been illustrated which was successful to alter impaired $P$. aeruginosa phagocytosis. A possible intrinsic Rab5 activity cycle was established by Rin1 protein over-expression in conjunction with endogenous Ras. Within the short period of early phagocytic vesicle formation, Rin1 interacts with activated Ras and potentiates Rab5 activity by Vps9 catalytic domain. Further analysis of in vivo interaction between ADP-ribosylated Ras and Rab5 with Rin1 could lead to a therapeutic alternative to rescue infamously impaired phagocytic activity in host and help combat chronic $P$. aeruginosa infection. 


\subsection{Figures legands}
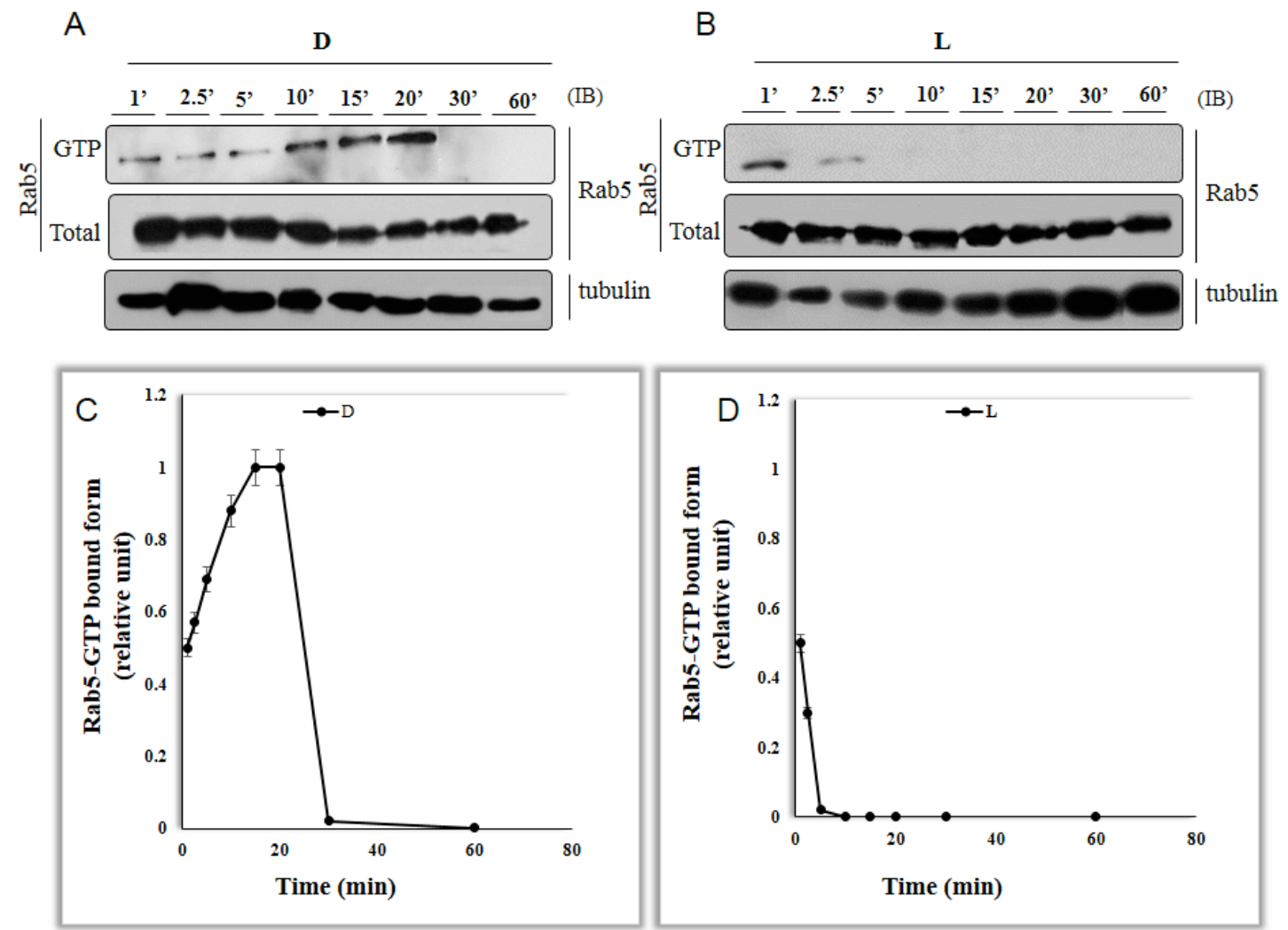

Figure 22: Shortened life span of Rab5-GTP bound form in the event of early endocytosis during live $P$. aeruginosa invasion.

J774-Eclone macrophage cells expressing pMX vector were incubated with heatinactivated, dead (D) P. aeruginosa (Panel A) or live (L) P. aeruginosa (panel B) at a ratio of 200:1 for 1 minute, 2.5 minutes, 5 minutes, 10 minutes, 15 minutes, 20 minutes, 30 minutes, and 60 minutes at $37^{\circ} \mathrm{C}$. Cells were then washed with ice-cold PBS, lysed and incubated with anti Rab5-GTP monoclonal antibody followed by protein $\mathrm{A} / \mathrm{G}$ agarose beads as described in Materials and Method. After incubation, the beads were washed and GTP-bound activated Rab5 was analyzed by immunoblotting with anti-Rab5. Top panels (A and B): Shows a representative immunoblot of samples probed with antiRab5 antibodies for total Rab5 and active Rab5 or anti-tubulin antibodies as control for total protein in cell lysate. Bottom panel (C and D) represents quantification of the course of activation of Rab5 during the described phagocytosis experiment. Data represent the mean \pm SEM of three independent experiments. 
A
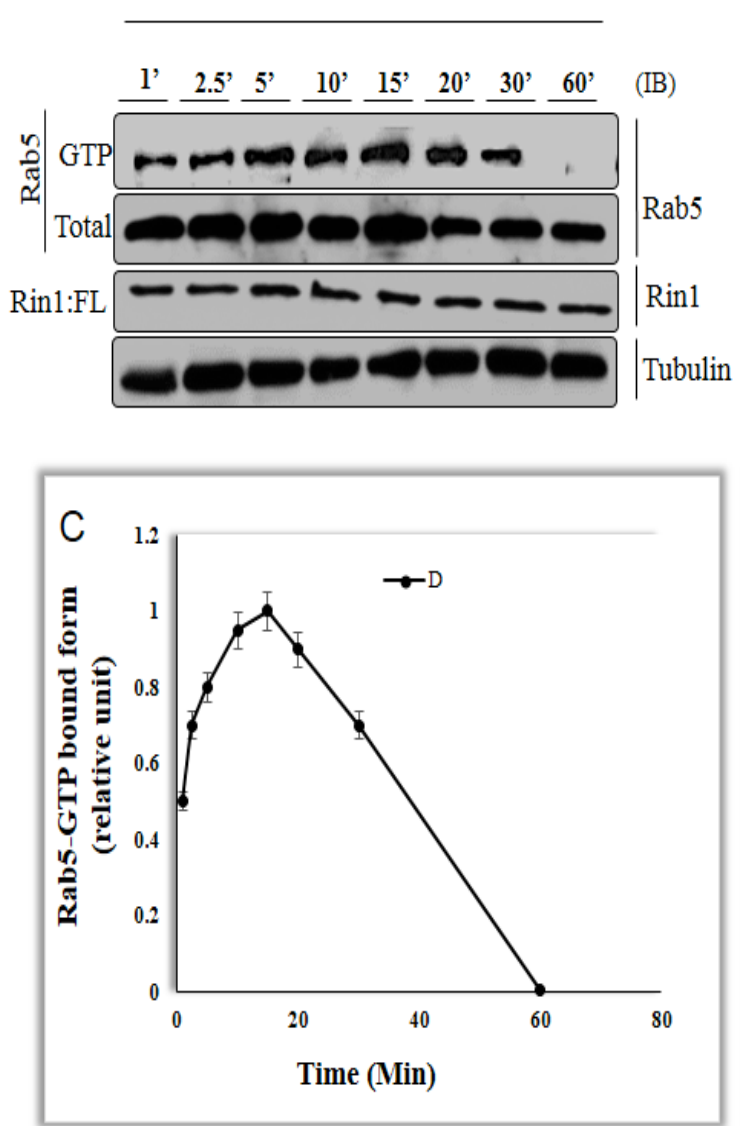

B
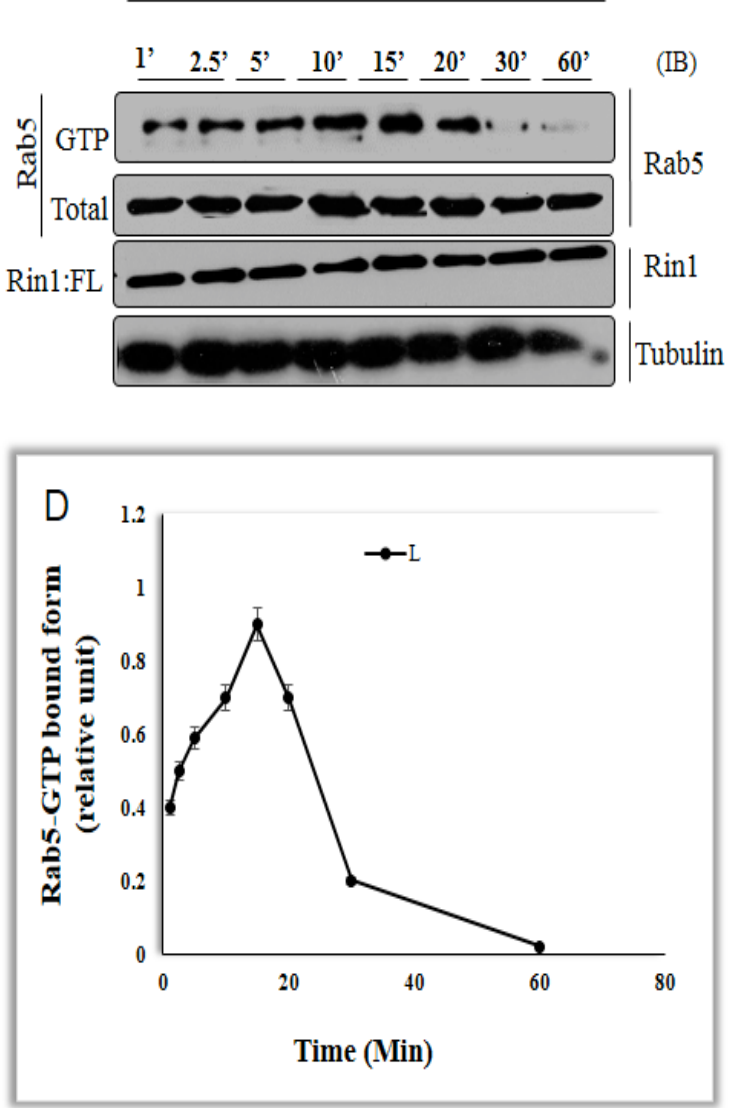

Figure 23: Effect of Rin1 FL, a Rab5-GEF on Rab5 activity during phagocytosis of P. aeruginosa.

(Panel A) Live (L) or (Panel B) heat-inactivated, dead (D) P. aeruginosa were incubated with cells expressing Rin1 FL, a Rab5-GEF at a MOI of 200:1 bacteria to cell. $P$. aeruginosa phagocytosis was assayed after incubation at $37^{\circ} \mathrm{C}$ for 1 minute, 2.5 minutes, 5 minutes, 10 minutes, 15 minutes, 20 minutes, 30 minutes, and 60 minutes respectively. Cells were then washed and analyzed for active GTP-Rab5 as previously described in Material and Methods. A representative immunoblot of samples probed with anti-Rab5, anti-Rin1 or anti-tubulin antibody is shown in the top panel. Bottom panels (C and D) represent quantification of the course of activation of Rab5 during the described phagocytosis experiment. Data represent the mean \pm SEM of three independent experiments. 

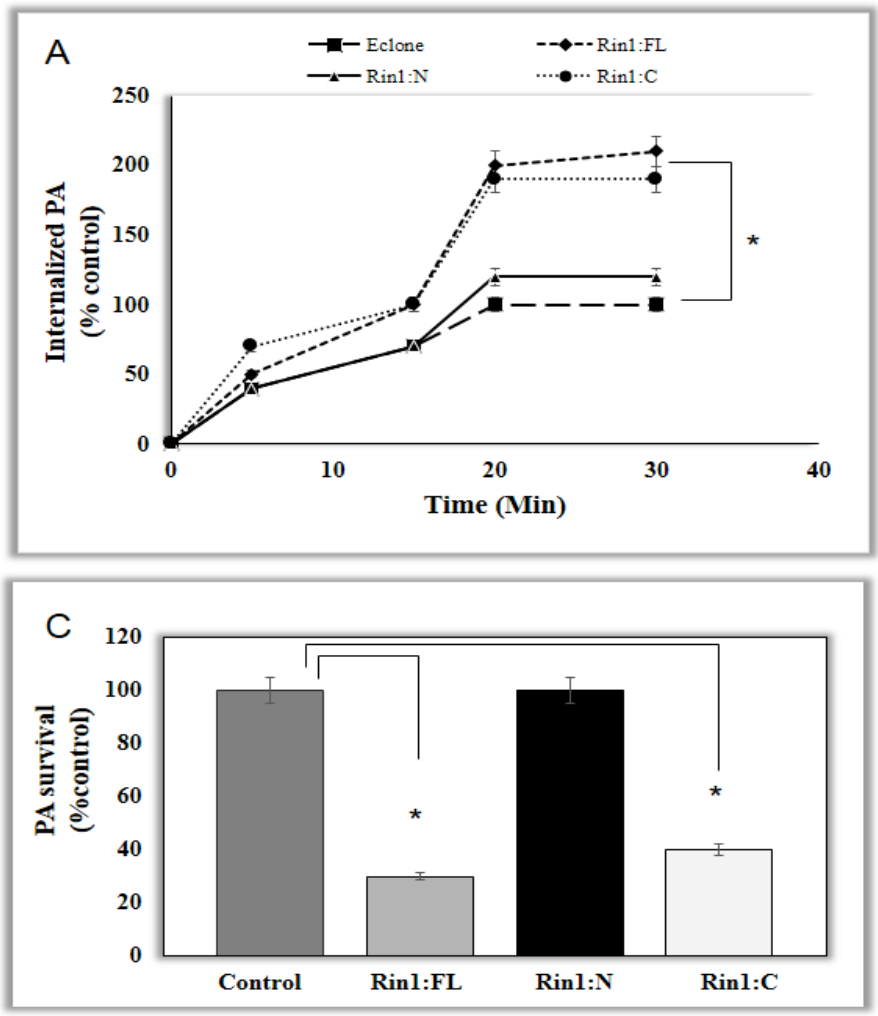

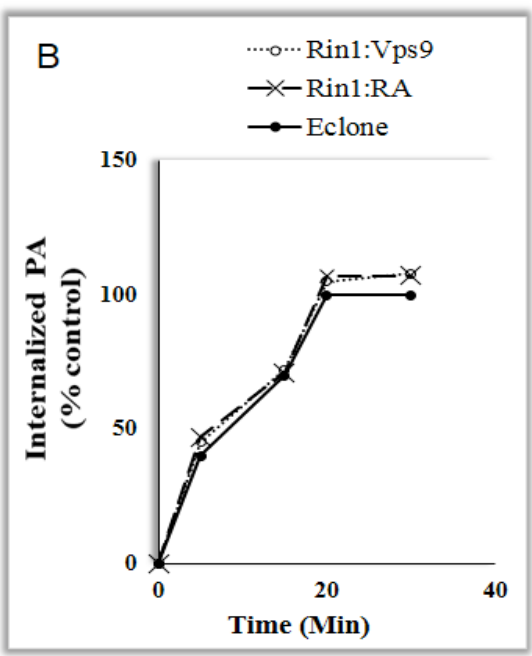

Figure 24: Determination of live (L) $P$. aeruginosa invasion and survival inside macrophage in presence of Rin1 FL and its domains.

(A) J774-Eclone cells expressing pMX (control) or Rin1 FL, Rin1 N or Rin1 C were incubated with live $P$. aeruginosa at a MOI of 200:1 for 0 minute, 5 minutes, 15 min, 20 minutes, and 30 minutes at $37^{\circ} \mathrm{C}$. After incubation, the phagocytic index (Internalized PA) was determined and is expressed as percent phagocytosis of live bacteria by control cells at 30 minutes. (B) Further, Rin1 Vps9 and Rin1 RA over-expressing J774-Eclone cells were subjected phagocytosis for 0 minute, 5minutes, 15minutes, 20 minutes, and 30 minutes respectively using live $P$. aeruginosa at a MOI of 200:1. Phagocytic index was determined essentially as described in Material Methods section. Phagocytic index (Internalized PA) is expressed as percent phagocytosis of live bacteria by control cells at 30 min. (C) J774-Eclone cells expressing pMX (control) or Rin1 FL, Rin1 N or Rin1 C were incubated with live $P$. aeruginos $a$ at a ratio of $200: 1$ for 30 minutes at $37^{\circ} \mathrm{C}$. which was followed by antibiotic protection assay as described in the Material and Methods and internalized bacteria were chased for additional 30 minutes and the survival of internalized bacteria were measured enumerating the colonies on agar plate. $(*)$ represents statistically significant difference from the control group $(P<0.05)$. 

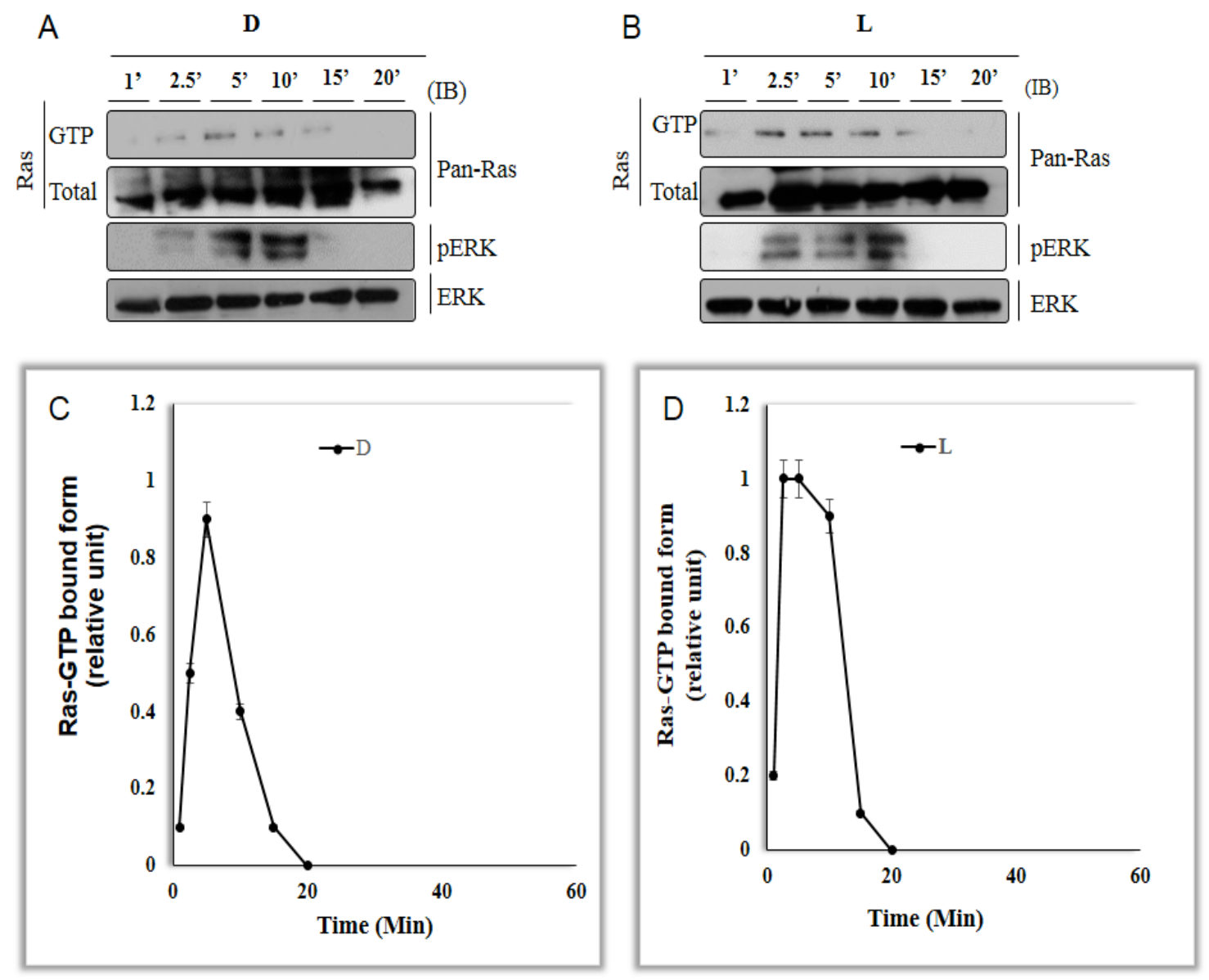

Figure 25: Activation of Ras during phagocytosis of $\boldsymbol{P}$. aeruginosa.

J774-Eclone cells expressing pMX were incubated with live (L) (Panel A) or heatinactivated (D) (Panel B) P. aeruginosa as described previously, and Ras activation was assessed using the RBD-GST pull-down assay as described in Material Methods. As a control, beads were only coated with GST and incubated with cell lysate. Top panels (A and B) show a representative immunoblot of samples probed with anti Pan-Ras antibody for GTP bound active Ras or total Ras in the cell lysate and as a control phosphorylated ERK (pERK) and total ERK was also probed in the cell lysate. Bottom panels (C and D) represent quantification of the course of activation of Ras during the described phagocytosis experiment. Data represent the mean \pm SEM of three independent experiments. 

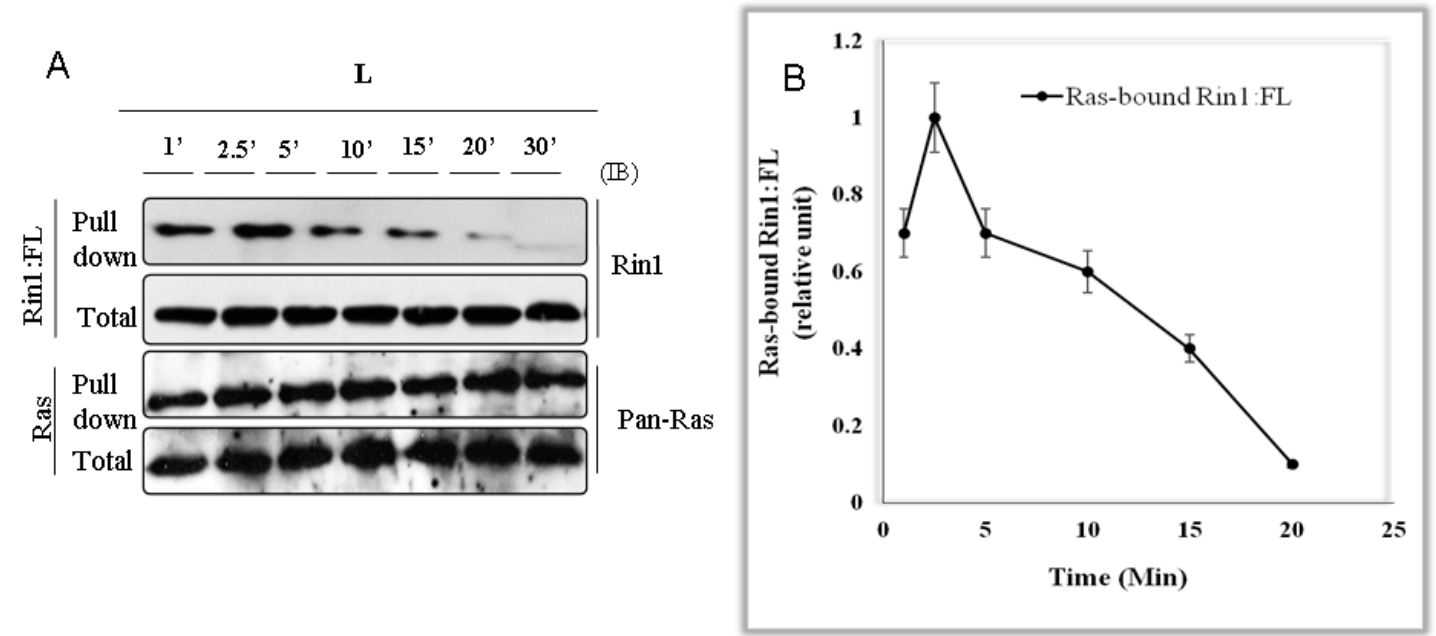

Figure 26: Rin 1 binds to Ras during phagocytosis of $P$. aeruginosa.

J774-Eclone cells expressing Rin1: FL were incubated with live (L) P. aeruginosa as described in the Materials and Methods and Ras was pulled down using monoclonal anti pan-Ras antibody also as described in the Materials and Methods. Finally, Ras bound Rin1: FL was probed using polyclonal anti Rin1 antibody. Top panel (A) represents the immunoblot of samples probed for pulled down Rin1: FL and total Rin1 FL in the cell lysates as well as pulled down Ras and total Ras in the cell lysates during the phagocytosis experiment. Bottom panel (B) represents quantification of pulled down Ras bound Rin1 during the described phagocytosis experiment. Data represent the mean \pm SEM of three independent experiments. 

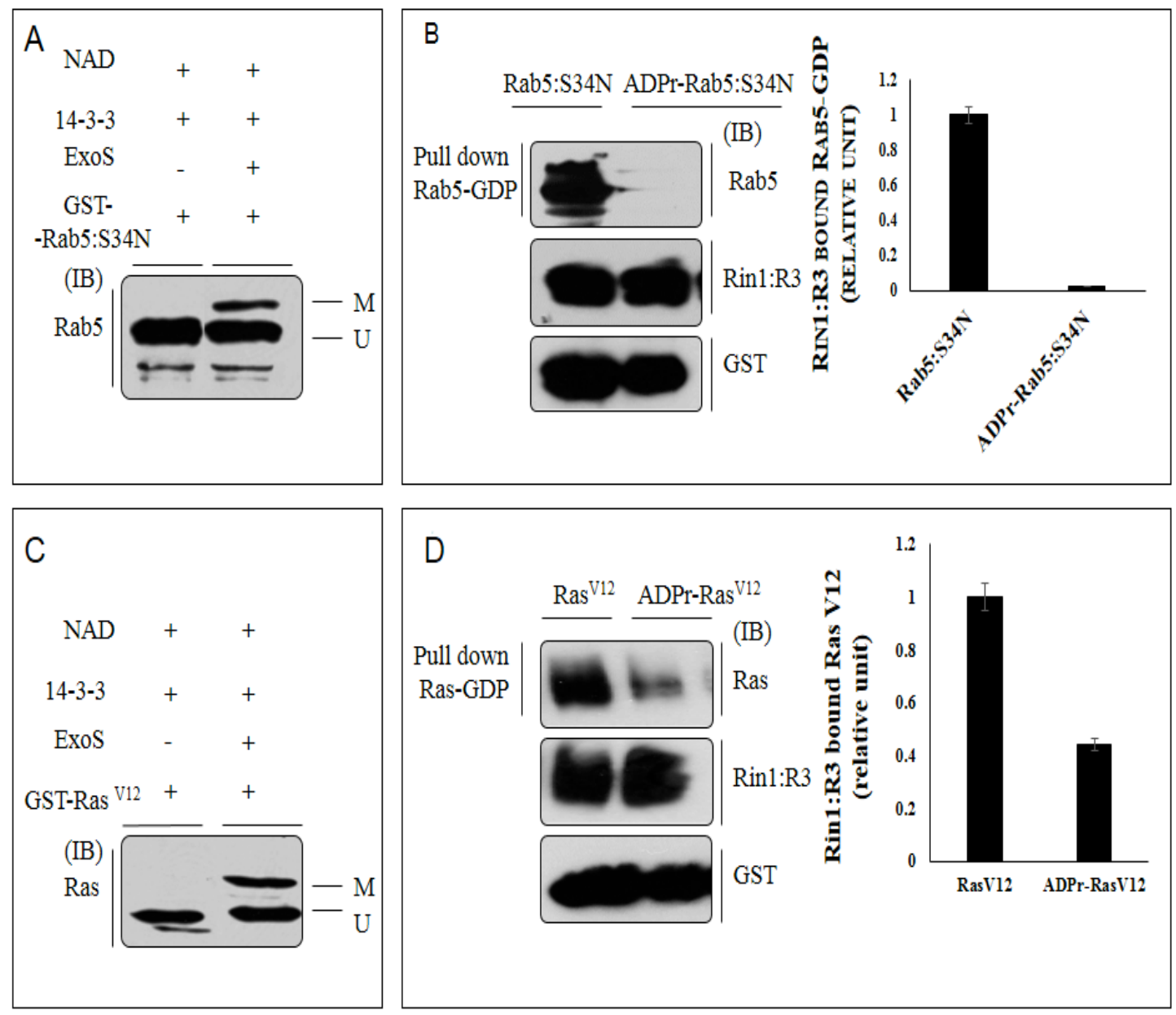

Figure 27: ADP-ribosylated Ras and Rab5 interaction with Rin1 R3 in vitro.

(A) ADP-ribosylation by ExoS alters the electrophoretic mobility of Rab5:S34N. Rab5:S34N was ADP-ribosylated by ExoS in the presence (+) or absence (-) of the indicated reagents. The band with the fast electrophoretic mobility corresponds to unmodified (u) Rab5:S34N, whereas the band with slow electrophoretic mobility corresponds to ADP-ribosylated (m) Rab5:S34N. A 50 $\mu 1$ reaction mixture of HisRab5:S34N proteins (1mM), His-ExoS (0.8mM), GST-14-3-3 (0.8mM), 0.2M Na acetate (pH 6.0), 1 mM Magnesium chloride and $10 \mathrm{mM}$ Biotinylated NAD was incubated at $20^{\circ} \mathrm{C}$ for 2 hours. Electrophoretic mobility shift was analyzed by immunoblotting (IB). (B) 3mM of ADP-ribosylated Rab5:S34N was added to immobilized GST-Rin1 C for 30 minutes at $4{ }^{\circ} \mathrm{C}$ in a total volume of $75 \mu$. Samples were centrifuged and pellets were analyzed in immunoblot (IB) probed with anti-Rab5-antibody, anti-Rin1-antibody and anti-GST-antibody. (C) Altered electrophoretic mobility of ADPR-ribosylated Ras:G12V $\left(\mathrm{Ras}^{\mathrm{v} 12}\right)$ by ExoS in the presence (+) or absence (-) of the indicated reagents. The band with the fast electrophoretic mobility corresponds to unmodified (u) Ras:G12V and the band with slow electrophoretic mobility corresponds to ADP-ribosylated (m) Ras:G12V. ADP-ribosylation reaction for His- Ras:G12V were run in a $50 \mu 1$ reaction mixture of 
0.2M Tris-acetate, $\mathrm{pH} 6.0,1 \mathrm{mM} \mathrm{MgCl} 2,10 \mathrm{mM}$ Biotinylated NAD, 0.2mM 14-3-3, 0.5 $\mathrm{mM}$ His-H- Ras:G12V and $0.2 \mathrm{mM}$ ExoS at $20^{\circ} \mathrm{C}$ for $2 \mathrm{~h}$. (D) $3 \mathrm{mM}$ of ADP-ribosylated His- Ras:G12V was added to immobilized GST-Rin1 C for 30 minutes at $4{ }^{\circ} \mathrm{C}$ in a total volume of $75 \mu$. Samples were centrifuged and pellets were analyzed in immunoblot (IB) probed with anti-Rab5-antibody, anti-Rin1-antibody and anti-GST-antibody. 


\subsection{References}

Alix, E., S. Mukherjee and C. R. Roy (2011). "Subversion of membrane transport pathways by vacuolar pathogens." J Cell Biol 195(6): 943-952.

Allen, L. A., C. Yang and J. E. Pessin (2002). "Rate and extent of phagocytosis in macrophages lacking vamp3." J Leukoc Biol 72(1): 217-221.

Angus, A. A., D. J. Evans, J. T. Barbieri and S. M. Fleiszig (2010). "The ADPribosylation domain of Pseudomonas aeruginosa ExoS is required for membrane bleb niche formation and bacterial survival within epithelial cells." Infect Immun 78(11): 4500-4510.

Arora, P. D., M. W. Chan, R. A. Anderson, P. A. Janmey and C. A. McCulloch (2005). "Separate functions of gelsolin mediate sequential steps of collagen phagocytosis." Mol Biol Cell 16(11): 5175-5190.

Barbieri, M. A., S. Fernandez-Pol, C. Hunker, B. H. Horazdovsky and P. D. Stahl (2004). "Role of rab5 in EGF receptor-mediated signal transduction." Eur J Cell Biol 83(6): 305314.

Barbieri, M. A., A. D. Kohn, R. A. Roth and P. D. Stahl (1998). "Protein kinase B/akt and rab5 mediate Ras activation of endocytosis." J Biol Chem 273(31): 19367-19370.

Barbieri, M. A., C. Kong, P. I. Chen, B. F. Horazdovsky and P. D. Stahl (2003). "The SRC homology 2 domain of Rin1 mediates its binding to the epidermal growth factor receptor and regulates receptor endocytosis." J Biol Chem 278(34): 32027-32036.

Barbieri, M. A., G. Li, M. I. Colombo and P. D. Stahl (1994). "Rab5, an early acting endosomal GTPase, supports in vitro endosome fusion without GTP hydrolysis." J Biol Chem 269(29): 18720-18722.

Barbieri, M. A., G. G. Tall, B. F. Horazdovsky and P. D. Stahl (2001). "Receptormediated endocytosis: EGF/Ras-activated endocytosis is mediated by Rab5 and the Rab5 guanine nucleotide exchange activity of RIN1." Faseb Journal 15(5): A1162-A1162.

Botelho, R. J., R. E. Harrison, J. C. Stone, J. F. Hancock, M. R. Philips, J. Jongstra-Bilen, D. Mason, J. Plumb, M. R. Gold and S. Grinstein (2009). "Localized diacylglyceroldependent stimulation of Ras and Rap1 during phagocytosis." J Biol Chem 284(42): 28522-28532.

Brumell, J. H. and M. A. Scidmore (2007). "Manipulation of rab GTPase function by intracellular bacterial pathogens." Microbiol Mol Biol Rev 71(4): 636-652.

Bucci, C., R. G. Parton, I. H. Mather, H. Stunnenberg, K. Simons, B. Hoflack and M. Zerial (1992). "The small GTPase rab5 functions as a regulatory factor in the early endocytic pathway." Cell 70(5): 715-728. 
Burd, C. G., P. A. Mustol, P. V. Schu and S. D. Emr (1996). "A yeast protein related to a mammalian Ras-binding protein, $\mathrm{Vps} 9 \mathrm{p}$, is required for localization of vacuolar proteins." Mol Cell Biol 16(5): 2369-2377.

Carney, D. S., B. A. Davies and B. F. Horazdovsky (2006). "Vps9 domain-containing proteins: activators of Rab5 GTPases from yeast to neurons." Trends Cell Biol 16(1): 2735.

Clarke, M., U. Engel, J. Giorgione, A. Muller-Taubenberger, J. Prassler, D. Veltman and G. Gerisch (2010). "Curvature recognition and force generation in phagocytosis." BMC Biol 8: 154.

Colicelli, J., C. Nicolette, C. Birchmeier, L. Rodgers, M. Riggs and M. Wigler (1991). "Expression of three mammalian cDNAs that interfere with RAS function in Saccharomyces cerevisiae." Proc Natl Acad Sci U S A 88(7): 2913-2917.

Coste, I., K. Le Corf, A. Kfoury, I. Hmitou, S. Druillennec, P. Hainaut, A. Eychene, S. Lebecque and T. Renno (2010). "Dual function of MyD88 in RAS signaling and inflammation, leading to mouse and human cell transformation." J Clin Invest 120(10): 3663-3667.

David, M. D., C. L. Cochrane, S. K. Duncan and J. W. Schrader (2005). "Pure lipopolysaccharide or synthetic lipid A induces activation of p21Ras in primary macrophages through a pathway dependent on Src family kinases and PI3K." J Immunol 175(12): 8236-8241.

Ehrhardt, A., M. D. David, G. R. Ehrhardt and J. W. Schrader (2004). "Distinct mechanisms determine the patterns of differential activation of H-Ras, N-Ras, K-Ras 4B, and M-Ras by receptors for growth factors or antigen." Mol Cell Biol 24(14): 6311-6323.

Ehrhardt, A., G. R. Ehrhardt, X. Guo and J. W. Schrader (2002). "Ras and relatives--job sharing and networking keep an old family together." Exp Hematol 30(10): 1089-1106.

Galvis, A., V. Balmaceda, H. Giambini, A. Conde, Z. Villasana, M. W. Fornes and M. A. Barbieri (2009). "Inhibition of early endosome fusion by Rab5-binding defective Ras interference 1 mutants." Arch Biochem Biophys 482(1-2): 83-95.

Galvis, A., H. Giambini, Z. Villasana and M. A. Barbieri (2009). "Functional determinants of ras interference 1 mutants required for their inhbitory activity on endocytosis." Exp Cell Res 315(5): 820-835.

Ganesan, A. K., T. S. Vincent, J. C. Olson and J. T. Barbieri (1999). "Pseudomonas aeruginosa exoenzyme $\mathrm{S}$ disrupts Ras-mediated signal transduction by inhibiting guanine nucleotide exchange factor-catalyzed nucleotide exchange." J Biol Chem 274(31): 21823-21829. 
Gorvel, J. P., P. Chavrier, M. Zerial and J. Gruenberg (1991). "rab5 controls early endosome fusion in vitro." Cell 64(5): 915-925.

Han, L. and J. Colicelli (1995). "A human protein selected for interference with Ras function interacts directly with Ras and competes with Raf1." Mol Cell Biol 15(3): 13181323.

Han, L., D. Wong, A. Dhaka, D. Afar, M. White, W. Xie, H. Herschman, O. Witte and J. Colicelli (1997). "Protein binding and signaling properties of RIN1 suggest a unique effector function." Proc Natl Acad Sci U S A 94(10): 4954-4959.

Horiuchi, H., R. Lippe, H. M. McBride, M. Rubino, P. Woodman, H. Stenmark, V. Rybin, M. Wilm, K. Ashman, M. Mann and M. Zerial (1997). "A novel Rab5 GDP/GTP exchange factor complexed to Rabaptin-5 links nucleotide exchange to effector recruitment and function." Cell 90(6): 1149-1159.

Jansson, A. L., L. Yasmin, P. Warne, J. Downward, R. H. Palmer and B. Hallberg (2006). "Exoenzyme S of Pseudomonas aeruginosa is not able to induce apoptosis when cells express activated proteins, such as Ras or protein kinase B/Akt." Cell Microbiol 8(5): 815-822.

Knight, D. A., V. Finck-Barbancon, S. M. Kulich and J. T. Barbieri (1995). "Functional domains of Pseudomonas aeruginosa exoenzyme S." Infect Immun 63(8): 3182-3186.

Kortholt, A. and P. J. van Haastert (2008). "Highlighting the role of Ras and Rap during Dictyostelium chemotaxis." Cell Signal 20(8): 1415-1422.

Li, G., C. D'Souza-Schorey, M. A. Barbieri, J. A. Cooper and P. D. Stahl (1997). "Uncoupling of membrane ruffling and pinocytosis during Ras signal transduction." J Biol Chem 272(16): 10337-10340.

Li, J. D., W. Feng, M. Gallup, J. H. Kim, J. Gum, Y. Kim and C. Basbaum (1998). "Activation of NF-kappaB via a Src-dependent Ras-MAPK-pp90rsk pathway is required for Pseudomonas aeruginosa-induced mucin overproduction in epithelial cells." Proc Natl Acad Sci U S A 95(10): 5718-5723.

Mustafi, S., N. Rivero, J. C. Olson, P. D. Stahl and M. A. Barbieri (2013). "Regulation of Rab5 Function during Phagocytosis of Live Pseudomonas aeruginosa in Macrophages." Infect Immun 81(7): 2426-2436.

Mustafi, S., N. Rivero, J. C. Olson, P. D. Stahl and M. A. Barbieri (2013). "Regulation of Rab5 function in macrophages during phagocytosis of live Pseudomonas aeruginosa." Infect Immun.

Ponting, C. P. and D. R. Benjamin (1996). "A novel family of Ras-binding domains." Trends Biochem Sci 21(11): 422-425. 
Raoust, E., V. Balloy, I. Garcia-Verdugo, L. Touqui, R. Ramphal and M. Chignard (2009). "Pseudomonas aeruginosa LPS or flagellin are sufficient to activate TLRdependent signaling in murine alveolar macrophages and airway epithelial cells." PLoS One 4(10): e7259.

Roberts, R. L., M. A. Barbieri, J. Ullrich and P. D. Stahl (2000). "Dynamics of rab5 activation in endocytosis and phagocytosis." J Leukoc Biol 68(5): 627-632.

Sasaki, A. T., C. Janetopoulos, S. Lee, P. G. Charest, K. Takeda, L. W. Sundheimer, R. Meili, P. N. Devreotes and R. A. Firtel (2007). "G protein-independent Ras/PI3K/F-actin circuit regulates basic cell motility." J Cell Biol 178(2): 185-191.

Shields, J. M., K. Pruitt, A. McFall, A. Shaub and C. J. Der (2000). "Understanding Ras: 'it ain't over 'til it's over'." Trends Cell Biol 10(4): 147-154.

Tall, G. G., M. A. Barbieri, P. D. Stahl and B. F. Horazdovsky (2001). "Ras-activated endocytosis is mediated by the Rab5 guanine nucleotide exchange activity of RIN1." Dev Cell 1(1): 73-82.

Tall, G. G., M. A. Barbieri, P. D. Stahl and B. F. Horazdovsky (2001). "Ras-activated endocytosis is mediated by the Rab5 guanine nucleotide exchange activity of RIN1." Developmental Cell 1(1): 73-82.

Vincent, T. S., J. E. Fraylick, E. M. McGuffie and J. C. Olson (1999). "ADP-ribosylation of oncogenic Ras proteins by Pseudomonas aeruginosa exoenzyme S in vivo." Mol Microbiol 32(5): 1054-1064.

Wang, Y., R. T. Waldron, A. Dhaka, A. Patel, M. M. Riley, E. Rozengurt and J. Colicelli (2002). "The RAS effector RIN1 directly competes with RAF and is regulated by 14-3-3 proteins." Mol Cell Biol 22(3): 916-926. 


\section{Chapter 5}

Antimicrobial activity of Dehydroleucodine against Pseudomonas aeruginosa 


\subsection{Abstract}

The increasing resistance of Pseudomonas aeruginosa to conventional treatments demands the search for novel therapeutic strategies. Dehydroleucodine (DhL), a secondary metabolite from Artemisia douglasiana, has been documented as antimicrobial agent against Helicobacter pylori and Leishmania Mexicana and trypanosome cruzi. In this study, the antimicrobial activity of $\mathrm{DhL}$ against $P$. aeruginosa was examined. In vitro analysis antimicrobial activity of $\mathrm{DhL}$ was determined against $P$. aeruginosa strains PAO1, PA103 and PA14 in reference to growth and various virulence factors. Results showed that DhL was active against each strain where, PAO1 and PA103 showed higher susceptibility (MIC $0.48 \mathrm{mg} / \mathrm{ml}$ ) as compared to PA14 (MIC $0.96 \mathrm{mg} / \mathrm{ml}$ ). Also, when PAO1 strain of was grown in the presence of $\mathrm{DhL}\left(\mathrm{MIC}_{50}, 0.12 \mathrm{mg} / \mathrm{ml}\right)$, a delay in the generation time was noticed along with significant inhibition in LasA protease activity and LasB elastase activity as well as Pyocyanin secretion. ExoS, a Type III secretion effector was found to be absent from $\mathrm{DhL}$ treated PAO1 strain indicating inhibitory effect of DhL against Type III secretion effectors. Interruption in biofilm attachment phase was also observed in a stationary culture of PAO1 treated with $\mathrm{DhL}$. DhL treatment positively controlled P. aeruginosa mediated apoptosis in J774-Eclone macrophage cell line. Analysis under Electron microscope revealed that the cellular integrity of $P$. aeruginosa post $\mathrm{DhL}$ treatment was preserved. Although, the exact molecular mechanism is still under speculation, the antimicrobial activity demonstrated by $\mathrm{DhL}$ may be a useful alternative strategy in prevention and eradication of $P$. aeruginosa infection. 


\subsection{Introduction}

Pseudomonas aeruginosa is a Gram-negative opportunistic pathogen with a high prominence of intrinsic antibiotic resistance (Hancock and Speert 2000; Mesaros, Nordmann et al. 2007). Pseduomonas aeruginosa resistant strains are commonly found as a secondary infection in immune-compromised patients with cystic fibrosis, COPD, AIDS, Cancer and even among diabetics (Rubin Grandis, Branstetter et al. 2004; Krcmery, Koprnova et al. 2006; Gomes, Machado et al. 2011; Engler, Muhlemann et al. 2012; Hogardt and Heesemann 2013) leaving serious blood stream infection with significant mortality and healthcare cost (Weinstein, Towns et al. 1997; Wisplinghoff, Seifert et al. 2003). The success in establishing P. aeruginosa pathogenecity is largely due to formation of intractable biofilms and secretion of myriads of virulent factors including LasA protease, LasB elastase, pyocyanin, pyoverdin, Type III secretion (T3S) effectors and alginate (Kessler, Safrin et al. 1997; Lyczak, Cannon et al. 2002; McIver, Kessler et al. 2004; Lee, Smith et al. 2005; Hauser 2009). Unfortunately, selection of the most appropriate antibiotic is complicated due to the ability of $P$. aeruginosa to develop resistance to multiple classes of antibiotics. Pseudomonas aeruginosa can develop resistance to antibacterial because of the low permeability of its outer membrane (Livermore 1984), the constitutive expression of various efflux pumps (Livermore 2001)

and the naturally occurring chromosomal AmpC b-lactamase, giving it sensitivity towards penicillin $\mathrm{G}$; aminopenicillins, first and second generation cephalosporins (Nordmann and Guibert 1998). Pseduomonas aeruginosa easily acquires additional resistance mechanisms, which leads to serious therapeutic problems (Micek, Lloyd et al. 2005). Currently anti-Pseudomonas treatments include higher-than-usual doses of b- 
lactam, fluoroquinolones and amino glycosides which possess a high degree of toxicity and very low eradication rate (Pedersen 1992; Hauser and Sriram 2005). The dearth of successful antibiotics to completely control $P$. aeruginosa infection makes it crucial to find alternatives to currently available drugs. Since pathogenicity in $P$. aeruginosa is regulated by several secretory-system mediated cell-to-cell communications, inhibition of this systems can cause attenuation of virulence and protect against infection (Hentzer, Wu et al. 2003; Smith and Iglewski 2003; Adonizio, Kong et al. 2008). Artemisia, the largest diverse genera of Asteraceae family possesses medicinally valuable essential oils and secondary metabolites (Bhakuni 2001; Choi, Park et al. 2013; Kim, Jung et al. 2013). Many studies indicate antimicrobial activity in Artemisia spp (Kalemba, Kusewicz et al. 2002; Ramezani, Fazli-Bazzaz et al. 2004; Benli, Kaya et al. 2007; Mahboubi and Kazempour 2009; Ahameethunisa and Hopper 2010). Artemisia douglasiana (A. douglasiana) documented as 'matico' in Argentina is popular as a preventive folk medicine (Giordano, Guerreiro et al. 1990; Ariza Espinar 1992). Dehydroleucodine (DhL), a sesquiterpene lactone of the guaianolide group, which also contains a $\alpha$ methylene- $\gamma$-lactone ring in its molecule (figure 28), is the principle active secondary metabolite in A. douglasiana (Alicia B. Penissi 2006). DhL was first isolated from Lidbeckia pectinata (Bohlmann and Zdero 1972). The aerial parts of A. douglasiana Besser are also rich in $\mathrm{DhL}$ (Giordano, Guerreiro et al. 1990). The DhL has cytoprotective activity (Guardia, Guzman et al. 1994; Wendel, Maria et al. 1999; Wendel, Maria et al. 2008) as well as antimicrobial activity (such as against Leishmania mexicana and Helicobacter pylori (Barrera, Jimenez-Ortiz et al. 2008; Vega, Wendel et al. 2009). DhL inhibited cell proliferation (Polo, 2007) and growth of Trypanosome cruzi in culture 
(Brengio, Belmonte et al. 2000). Some studies showed anti-Pseudomonas activity in essential oil from A. douglasiana (Setzer, Vogler et al. 2004).

This report established the antimicrobial effects of $\mathrm{DhL}$ against $P$. aeruginosa in terms of attenuating growth and arresting virulent factors.

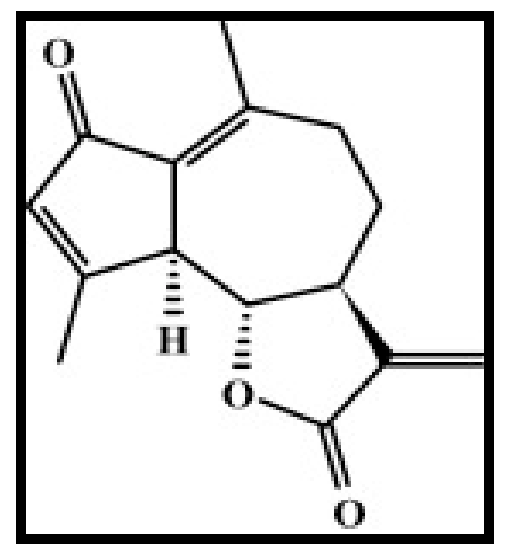

Figure 28: Chemical structure of Dehydroleucodine isolated from Artemisa douglasiana.

\subsection{Material and Methods}

\subsubsection{Materials}

Dehydroleucodine (DhL) was extracted from A. douglasiana as previously (Giordano, Guerreiro et al. 1990; Priestap, Abboud et al. 2011). All chemicals and reagents were purchased from Sigma-Aldrich (St. Louis, MO), unless otherwise indicated. Primary and secondary antibodies used in immunoblotting were purchased from Cell Signaling Technology Inc. (Danvers, MA). Culture supplies were purchased from Invitrogen Life Technologies (Carlsbad, CA).

\subsubsection{Bacterial Strain}

Prototypic P. aeruginosa strains used in this study are PAO1 (Holloway and Morgan 1986), clinical isolates PA103 and PA14, (kindly provided by Dr. Dara Frank, Wisconsin 
school of medicine) and P. aeruginosa strain PA103 $\Delta \mathrm{U} \Delta \mathrm{T}$, expressing pUCP plasmidencoded ExoS tagged with a hem-agglutinin epitope (ExoS-HA) (kindly provided by Dr. Joan, C. Olson, West Virginia University. In addition, Staphylococcus aureus (ATCC 12600) was used in the LasA assay. Cells were maintained in Lauria Broth (LB) (Life Technologies, Carlsbad, CA) or Agrobacterium (AB) minimum medium (Bio-world, Visalia, CA.) to which glucose and casein amino acids [20\%,wt/vol] were added.

\subsubsection{Determination of Minimum Inhibitory Concentration}

The antimicrobial activity of DhL was determined by a previously described micro dilution broth assay (Woolfrey, Fox et al. 1982). The minimum inhibitory concentration (Micek, Lloyd et al. 2005) of DhL for PA01, PA103 and PA14 strains of P. aeruginosa were determined. Serial doubling dilutions of $\mathrm{DhL}$ was made, ranging from $2.24 \mathrm{mg} / \mathrm{ml}$ $0.224 \mu \mathrm{g} / \mathrm{ml}$. A drop $(0.02 \mathrm{ml})$ of standard inoculums $(0.5$ McFarland $)$ of all three $P$. aeruginosa strains were introduced to desired volume of growth medium and incubated at $37^{\circ} \mathrm{C}$ for 24 hours. MIC was interpreted as the least concentration with no observable turbidity. $\mathrm{MIC}_{90}$ and $\mathrm{MIC}_{50}$ were determined as the concentration of $\mathrm{DhL}$ that inhibited growth by $90 \%$ and $50 \%$ respectively. Controls were set up, such as, sterility control: LB

broth only, viability control: LB broth and test bacteria, positive control: LB broth with Gentamycine and the test bacteria. They were incubated at $37^{\circ} \mathrm{C}$ overnight.

\subsubsection{DhL treatment (threshold concentration)}

For each assay $0.12 \mathrm{mg} / \mathrm{ml}\left(\mathrm{MIC}_{50}\right)$ of $\mathrm{DhL}$ was added to P. aeruginosa culture at early $\log$ phase unless otherwise stated. 


\subsubsection{Growth curves}

The effect of DhL on bacterial cell proliferation was determined by monitoring the growth curve of $P$. aeruginosa strain PAO1. Briefly, an overnight culture (in LB medium) of PAO1 was diluted to OD 0.05 in LB medium (control) or LB and DhL (0.12 $\mathrm{mg} / \mathrm{ml}$ ) and incubated at $37^{\circ} \mathrm{C}$ while shaking. The $\mathrm{OD}_{600}$ was monitored at 30 minute intervals until an $\mathrm{OD}_{600}$ of approximately 1.7 was obtained (approximately 8 hours). The $\mathrm{OD}_{600}$ of cultures with added $\mathrm{DhL}$ were normalized to the control $\mathrm{OD}_{600}$ at each time point to account for plant pigmentation. All $\mathrm{OD}_{600}$ measurements were verified at a $1 / 10$ dilution for greater accuracy.

\subsubsection{LasA staphylolytic activity}

Staphylolytic activity was determined spectro-photometricaly by measuring the decrease in absorbance at $595 \mathrm{~nm}$ of PAO1 culture supernatants mediated lysis of heat inactivated Staphylococcus aureus $(S$. aureus $)$ cells $(0.3 \mathrm{mg} / \mathrm{ml} ; 0.02 \mathrm{M}$ Tris-HCl, pH 8.5) (Kessler, Safrin et al. 1993). A $100 \mu 1$ aliquot of $P$. aeruginosa culture supernatant with or without DhL (after normalizing their concentration) was added to $900 \mu$ l of a boiled $S$. aureus suspension. The $\mathrm{OD}_{595}$ was determined after $0,5,10,20,30,45$, and 60 minutes respectively. Activity was expressed as the change in the $\mathrm{OD}_{595} /$ hour $/ \mu \mathrm{g}$ protein. One unit of activity was defined as the amount of enzyme that causes an $A_{595}$ decrease of 1 absorbance unit/min.

\subsubsection{LasB elastolytic assay}

Elastolytic activity in PAO1 culture fluids was determined by elastin Congo red (ECR) assay (Bjorn, Sokol et al. 1979). A $100 \mu$ aliquot of the AB medium culture supernatants from mid-log-phase with or without DhL treatment (concentration was 
normalized), was added to $900 \mu \mathrm{l}$ of ECR buffer (100 mM Tris, $1 \mathrm{mM} \mathrm{CaCl2,pH} \mathrm{7.5)}$ containing $20 \mathrm{mg}$ ECR. Tubes were incubated 18 hours at $37^{\circ} \mathrm{C}$ with rotation and then were placed on ice after $0.1 \mathrm{ml}$ of $0.12 \mathrm{M}$ EDTA was added. Insoluble ECR was removed by centrifugation, and the $\mathrm{OD}_{495}$ was measured. Absorption due to pigments produced by P. aeruginosa was corrected for by subtracting the $\mathrm{OD}_{495}$ of each sample that had been incubated in the absence of ECR. Cell-free $\mathrm{AB}$ medium alone and $\mathrm{AB}$ medium with $\mathrm{DhL}$ were used as negative controls.

\subsubsection{Polyvinyl chloride biofilm formation attachment assay}

The effect of DhL on the attachment phase of biofilm formation was measured by using the polyvinyl chloride biofilm formation assay (O'Toole and Kolter 1998). Briefly, overnight cultures of PAO1 were resuspended in fresh $\mathrm{AB}$ medium in the presence and the absence DhL. After 24 hours of incubation at $30^{\circ} \mathrm{C}$, the biofilms in the polyvinyl chloride micro titer plates were visualized by staining with a crystal violet solution. The plates were rinsed to remove planktonic cells, and the surface-attached cells were then quantified by solubilizing the dye in ethanol and measuring the absorbance at $\mathrm{OD}_{546}$.

\subsubsection{Pyocyanin assay}

Photometric pyocyanin assay was performed following previously described protocol by Grossowicz and others (Grossowicz, Hayat et al. 1957). $1.5 \mathrm{ml}$ static LB broth culture at mid $\log$ phase (with or without $\mathrm{DhL}$ ) was maintained at $37^{\circ} \mathrm{C}$ for 24 hours and then centrifuged at $14000 \mathrm{rpm}$ for 5 minutes. One $\mathrm{ml}$ of supernatants was mixed with $1.5 \mathrm{ml}$ of chloroform and left to separate. The blue organic layer was mixed with $1 \mathrm{ml}$ of $0.2 \mathrm{M}$ hydrochloric acid, whereby the blue mixture turning to a red form of pyocyanin moves 
into the aqueous layer. This was re-centrifuged prior to measuring the absorbance at 520 $\mathrm{nm}$ and pyocyanin formation was compared.

\subsubsection{Type III secretion assay}

Low calcium fractionation protocol (Lee, Smith et al. 2005) was adopted for the detection of (T3S) effectors. Briefly, P. aeruginosa PAO1, PA103 $\Delta \mathrm{U} \Delta \mathrm{T}$ expressing HAExoS and were grown overnight in LB and appropriate selection antibiotic. Bacteria were subcultured 1:1,000 in LB supplemented with 5 mM EGTA with or without DhL and grown for 6 hours at $37^{\circ} \mathrm{C}$ with aeration. Bacterial densities were determined at $\mathrm{OD}_{600}$ $\mathrm{nm}$. Bacteria were sedimented by centrifugation at 3,220 $\mathrm{g}$ for 15 minutes at $4^{\circ} \mathrm{C}$. Culture supernatant was collected, and proteins were precipitated with 5\% trichloroacetic acid and washed with ice cold acetone. Proteins were resuspended according to culture density and separated by sodium dodecyl sulfate-polyacrylamide gel electrophoresis (SDSPAGE). Proteins derived from PAO1 (with or without DhL) supernatant precipitates were run on a SDS-PAGE stained with Coomassie blue. Also proteins derived from PA103 $\Delta \mathrm{U} \Delta \mathrm{T}$ expressing HA-ExoS were transferred onto polyvinylidene difluoride (PVDF) membranes for detection of HA-ExoS by antibodies against HA.

\subsubsection{P. aeruginosa mediated apoptosis of Eclone cells: Hoechst staining of}

\section{condensed chromatin}

The effect of DhL treatment on P. aeruginosa mediated apoptosis of J774-Eclone mouse macrophage cell was monitored according to Jia et al, 2006 (Jia, Wang et al. 2006). J774-Eclone cell monolayer were plated from suspension culture 1 day prior to infection in DMEM supplemented with 5\% FBS (DMEM-5\%FBS). Cell monolayer $(\sim 5 \times$ $10^{5}$ cells per well; $>80 \%$ confluence) were washed with phosphate-buffered saline (PBS), 
mixed with PAO1 bacteria (grown in LB- DhL or only LB) at a multiplicity of infection (MOI) of 20 and incubated for 2 hours at $37^{\circ} \mathrm{C}$ in a $5 \% \mathrm{CO}_{2}$ incubator. The cells were washed with PBS to remove the non adhering bacteria. Fresh medium, DMEM-5\% FBS supplemented with $400 \mu \mathrm{g}$ of Gentamycine or Amikacin per ml, was added, and the cells were incubated for an additional 2 hours. As positive controls for the apoptosis, J774Eclone cells were incubated with $0.1 \mathrm{M}$ of Stourosporine (Fischer scientific, Waltham, MA) for 1 hour. Cells were washed once with PBS and stained with Hoechst 33258 (Molecular Probes, Inc., Eugene, OR) at $1 \mathrm{mg} / \mathrm{ml}$ for 10 minutes in the dark. Chromatin condensation was examined under the fluorescence microscope by using a DAPI $(4,6-$ diamidino-2-phenylindole) filter after stained cells were mounted onto slides using mounting medium.

\subsubsection{Electron Microscopy}

Bacterial cells (strain PAO1) were grown in presence or absence of $0.48 \mathrm{mg} / \mathrm{ml}$ of $\mathrm{DhL}$ and then resuspended in the fixative described by Mollenhauer and others (Mollenhauer, Morre et al. 1977). Fixed cells were dehydrated by grade of alcohol acetone and embedded in Epon 812 (Pelco Company). Thin sections were obtained in an Ultra microtome (Ultra cut, Leica), stained with led citrate and uranile and observed at 50 $\mathrm{Kv}$ in a Zeiss 900 electron microscope (Germany).

\subsubsection{Statistics}

All experiments were performed independently in triplicate and each experiment was repeated three times. Values represent the mean \pm SEM of three independent experiments. Data were analyzed by one-way analysis of variance, with a $P$ value of 0.05 being significant, by using the SPSS (Chicago, IL) statistical software package. 


\subsubsection{Western Blot Image J}

Image J64 was used to quantify Western blots after images were scanned at a grayscale amplification of $600 \mathrm{dpi}$.

\subsection{Results}

\subsubsection{Activity of DhL against Pseudomonas aeruginosa.}

For studying the antibacterial effects of $\mathrm{DhL}$, varying concentrations of $\mathrm{DhL}$ ranging from $2.24 \mathrm{mg} / \mathrm{ml}-0.224 \mu \mathrm{g} / \mathrm{ml}$ in growth media (LB) derived from a double dilution method on a 96 well plate was tested against $P$. aeruginosa strains PAO1, PA14 and PA103. $50 \%$ of PAO1 and PA103 population was inhibited at $0.12 \mathrm{mg} / \mathrm{ml}$ whereas 0.48 $\mathrm{mg} / \mathrm{ml}$ of DhL successfully inhibited $50 \%$ of PA14 population. It has also been observed that the minimum inhibitory concentration (Micek, Lloyd et al. 2005), which is defined as the lowest concentration of the DhL solution that inhibits growth of the microbial strain, was $0.48 \mathrm{mg} / \mathrm{ml}$ for PAO1 and PA103 as compared to $0.96 \mathrm{mg} / \mathrm{ml}$ for the more virulent strain PA14 (Table 1). Overall, DhL was effective against all strains of $P$. aeruginosa tested here, although strains PAO1 and PA103 seemed to be more susceptible to DhL than PA14 and in each case the effective concentration of DhL was much higher than potent antibiotic Gentamycine (Table 1).

\subsubsection{Electron Microscopy analysis of Pseudomonas aeruginosa cellular structure}

Pseduomonas aeruginosa strain PAO1 from an overnight culture was diluted to an OD of 0.05 and grown for an additional 3 hours with or without $0.48 \mathrm{mg} / \mathrm{ml}$ of $\mathrm{DhL}$. Cells were then prepared for EM studies as described in Material Methods. Results indicated that the ultra-structure of bacterial cell was clearly preserved and no structural differences could be established between treated and untreated groups (Figure 29). 


\subsubsection{Influence of threshold concentration of DhL on growth rate}

Further, results observed from growth studies with $P$. aeruginosa strain PAO1 indicated that with the decrease in $\mathrm{DhL}$ concentration in culture media, the growth phases were recovered in PAO1 culture (data not shown). Although treatment at $\mathrm{MIC}_{90}$ failed to portray normal growth curve (data not shown), at $\mathrm{MIC}_{50}$ prominent log and stationary phases were recovered (Figure 30). Noticeable differences in generation time were observed between 1-3 hours of growth (Mid-log phase) at $\mathrm{MIC}_{50}$ dose of $\mathrm{DhL}$ between treated and untreated PAO1 (Figure 30). The overall analysis suggested a positive antimicrobial effect of DhL on P. aeruginosa.

\subsubsection{Activity of DhL against Pseudomonas aeruginosa virulence factors: Elastase A} (LasA) and Elastase B (LasB)

To investigate the effect of DhL on LasA carrying elastase activity and LasB carrying protease activity from the repertoire of $P$. aeruginosa virulence factors, a lower dose of $\mathrm{DhL}\left(\mathrm{MIC}_{50}, 0.12 \mathrm{mg} / \mathrm{ml}\right)$ was selected as a threshold of antimicrobial activity. The effect of $\mathrm{DhL}$ on the activity of LasA and LasB proteases was determined in P. aeruginosa strain PAO1 (Figure 31A and 31B) as described in Material and Methods. The LasA staphylolytic protease is a $20-\mathrm{kDa}$ zinc metallo-endopeptidase (Kessler, Safrin et al. 1993). A significant decrease $(60 \pm 6 \%)$ in LasA activity was noticed after 60 minutes of incubation of $S$. aureus substrate along with the DhL treated PAO1 culture supernatant. PAO1 cultural supernatant in absence of $\mathrm{DhL}$ showed an exponentially increasing staphylolytic effect indicating protease activity when compared to $S$. aureus concentration in LB as a control (Figure 31A). As a control, similar concentration of DhL in LB was introduced to S. aureus to look for any lytic activity of $\mathrm{DhL}$ in the supernatant 
in absence of PAO1 (Data not shown). The LasB is also a zinc metallo-protease which is capable of destroying or inactivating a wide range of biological tissues and immunological agents (Bever and Iglewski 1988). There was a significant decrease of $75 \pm 5 \%$ in LasB activity when PAO1 was grown in the presence of $\mathrm{DhL}$ at $\mathrm{MIC}_{50}$ (Figure 31B). As a control, DhL and LB itself showed no elastase activity. In both experiments, PAO1 cultures grown in presence or absence of $\mathrm{DhL}$ were normalized so that the total number of bacteria remained the same. Thus, results indicated $\mathrm{DhL}$ mediated control on protease activity in live bacteria but not loss of activity due to loss of bacterial viability. In conclusion, DhL effectively inhibited both LasA and LasB activity in PAO1 population.

\subsubsection{DhL has an inhibitory effect on biofilm formation}

Pseduomonas aeruginosa has the ability to form biofilms, a partially quorum sensing controlled phenomenon (Davies 2003) in which cells are organized into layers and enmeshed in a matrix of mucoid polysaccharides (Costerton, Lewandowski et al. 1995). To determine if DhL could be used prophylactically to prevent biofilm formation, PAO1 strain (DhL treated or not) generated biofilms were spectro-photometrically assessed after the crystal violet staining (Figure 32). There was a significant decrease of $55 \pm 5 \%$ in biofilm formation compared to the control when strain PAO1 was grown in the presence of $\mathrm{DhL}\left(\mathrm{MIC}_{50}\right)$. Although, this experiment is unable to explain the exact mechanism of control, it is more likely that $\mathrm{DhL}$ has an effect directly on the bacterial cells rather than extracellular substances and mucoid layers of biofilm. 


\subsubsection{DhL alter pyocyanin production}

Psedomonas aeruginosa generates highly diffusible pigmented toxic secondary metabolites, known as phenazines which are critical for $P$. aeruginosa mediated killing of Caenorhabditis elegans and of mice in septicemia models (Mahajan-Miklos, Rahme et al. 2000). Pyocyanin secretion in PAO1 when grown in presence of $\mathrm{DhL}$ at $\mathrm{MIC}_{50}$ showed a substantial decrease in production ( $75 \pm 5 \%)$ compared to the control (Figure 33$)$.

\subsubsection{DhL inhibits Type III secretion effectors}

Pseduomonas aeruginosa strain PAO1 carrying T3S effectors ExoS, ExoT and ExoY, and effector less strain PA103 $\Delta \mathrm{U} \Delta \mathrm{T}$ : HA-ExoS were grown in an over night culture and then examined for the T3S effector secretion in presence or absence of DhL, essentially following the protocol by Lee and others, with a few modifications (Lee, Smith et al. 2005). Secreted proteins from T3SS in calcium-depleted culture supernatants treated with or without DhL were precipitated and separated by SDS-PAGE. ExoS is a major secreted protein of $P$. aeruginosa strain PAO1, corresponding to its predicted size $49 \mathrm{kDa}$ in coomassie blue stained SDS-PAGE gel (Figure 34). In calcium depleted media, presence of DhL seemed to control T3S effector secretion (Figure 34, top panel). To further confirm the presence of ExoS in the secretion profile, PA103 $\Delta \mathrm{U} \Delta \mathrm{T}$ : HA-ExoS, carrying hemaglutanin (HA) tagged ExoS (see Material Methods) was used in a similar experiment and detected with specific anti-HA antibody (Figure 34, bottom panel).

\subsubsection{DhL treated Pseudomonas aeruginosa and apoptosis}

Studies have suggested that T3S effectors from $P$. aeruginosa are responsible for inducing rapid apoptosis is macrophage cells and epithelial cells (Hauser and Engel 1999; Kaufman, Jia et al. 2000). Prior results in this study indicated down regulation of 
virulence factors in PAO1 when gown in DhL treated growth media. To follow up with the observation on T3S effectors, further investigations were conducted to observe the effect of DhL $\left(\mathrm{MIC}_{50}\right)$ treatment on P. aeruginosa strain PAO1 induced apoptosis. PAO1 cultures (with or without $\mathrm{DhL}$ treatment) were added to J774 Eclone cell monolayer at a MOI of 20:1. After 2 hours of incubation at $37^{\circ} \mathrm{C}$, cells were stained with Hoechst stain and apoptotic nuclei were observed under fluorescent microscope. The total percentage of apoptotic cell counted suggests that $\mathrm{DhL}$ treated PAO1 fail to induce apoptosis significantly compared to untreated PAO1 induced apoptosis (control). Almost $87 \pm 5 \%$ inhibition of apoptosis observed in PAO1 population previously treated with $\mathrm{DhL}$ when compared to the control population (Figure 35).

\subsection{Discussion}

Pseudomonas aeruginosa, an opportunistic pathogen often causing fatal infections in susceptible patients, has evolved a number of strategies by which bacterial factors affect host immunity and invade host cell (Buret and Cripps 1993). The LasA elastase and LasB protease play a major role in $P$. aeruginosa pathogenesis. The LasA have low elastolytic and staphylolytic activity (Kessler, Safrin et al. 1993) and also helps to increase LasB and other bacterial protease activity (Toder, Gambello et al. 1991). Proteolytic activity of LasA helps in bacterial invasion through epithelial tight junctions degrading the inhibitors of invasion in the host (Hoge 2010). The LasB elastase on the other hand is very crucial in Cystic Fibrosis lung infection (Voynow, Fischer et al. 2008) and known to degrade elastin and collagen in host (Heck, Morihara et al. 1986; Hamdaoui, WundBisseret et al. 1987; Kessler and Safrin 1988; Saulnier, Curtil et al. 1989). The LasB is also known to interact with host immune proteins (Schultz and Miller 1974; Bainbridge 
and Fick 1989; Heck, Alarcon et al. 1990). Pseudomonas aeruginosa is a biofilmforming bacterium. Biofilm formation by P. aeruginosa is intimately linked to cell-to-cell communication known as quorum sensing, in which small diffusible signaling molecules known as autoinducers regulate gene expression and increase protection against host immune responses (Costerton, Lewandowski et al. 1995; Dasgupta 1996; Davies 2003). Pseudomonas aeruginosa exhibit quorum sensing behavior using two distinct acylhomoserine lactone (Engler, Muhlemann et al. 2012) based pathways: the $r h l I / r h l R$ pathway using butyryl acyl homoserine lactone (C4-HSL), and the lasI/lasR pathway using 3-oxo-dodecanoyl homoserine lactone (3-oxo C12-HSL) (Pesci, Milbank et al. 1999). Biofilm-grown bacterial cells show increased resistance to anbiotics (Costerton, Lewandowski et al. 1995). Pseudomonas aeruginosa is the only organism known to produce the specific phenazine pyocyanin, a toxic secondary metabolite (Reyes, Bale et al. 1981) that have been previously shown to accelerate neutrophil apoptosis in vitro (Usher, Lawson et al. 2002). In animal infection models mimicking P. aeruginosa mediated acute human infections, such as burn wound, acute pneumonia, and corneal infection; it was shown that type III secretion (T3S) is an important virulence mechanism (Kudoh, Wiener-Kronish et al. 1994; Sawa, Ohara et al. 1998; Holder, Neely et al. 2001; Moss, Ehrmantraut et al. 2001). In 1998, Hauser and others illustrated that T3SS in $P$. aeruginosa induces apoptosis in epithelial cells and macrophage cells.

The aim of the present study was to assess the antimicrobial effect of DhL against $P$. aeruginosa growth and virulence factors. The PAO1 strain of P. aeruginosa is the standard laboratory strain as well as genetic reference strain, with a completely sequenced 6.3-Mb genome and 5,570 annotated open reading frame (ORFs) (Stover, 
Pham et al. 2000). The PA14 strain, a "multi host" pathogen is capable of infecting animals (in a burned mouse model), plants, insects, and nematodes (Rahme, Stevens et al. 1995; Mahajan-Miklos, Rahme et al. 2000). Both strains were able to initiate and maintain chronic infection in rat lung model (Kukavica-Ibrulj, Bragonzi et al. 2008). Exotoxin-A producing strain, PA103, is a cytotoxic strain of $P$. aeruginosa which is known to cause severe alveolar epithelial injury during infection (Kudoh, WienerKronish et al. 1994; Fleiszig, Zaidi et al. 1996). Virulent strains (PA14, PA103) and laboratory reference strain (PAO1) of $P$. aeruginosa were completely inhibited in vitro by $\mathrm{DhL}$ and the MICs vary from 0.48 to $0.96 \mathrm{mg} / \mathrm{ml}$. When PAO1 strain was grown in presence of a low dose of $\mathrm{DhL}\left(\mathrm{MIC}_{50}=0.12 \mathrm{mg} / \mathrm{ml}\right)$, the growth curve was recovered which indicated decreased bacterial killing, but the generation time was significantly reduced by $50 \pm 5 \%$. This suggested a possible role of $\mathrm{DhL}$ on bacterial cell cycle and slowing down of doubling time. The $\mathrm{DhL}$ treatment also inhibited several P. aeruginosa virulence factors at the threshold dose of $0.12 \mathrm{mg} / \mathrm{ml}$. The PAO1 cultural supernatant treated with or without $\mathrm{DhL}$ showed $60 \pm 6 \%$ decrease in LasA activity and $75 \pm 5 \%$ reduction in LasB activity. DhL treated PAO1 culture failed to demonstrate successful initial attachment phase during the course of biofilm formation and prolonged observation revealed disruption in biofilm formation compared to untreated PAO1 culture (data not shown). Effect of DhL was also poignant for $P$. aeruginosa pigment secretion and T3SS. Almost $85 \pm 7 \%$ of reduction in Pyocyanin formation and complete inhibition of T3S effector resulted from $\mathrm{DhL}$ treatment. Over all the results indicated a prominent regulation of $P$. aeruginosa growth and virulence due to $\mathrm{DhL}$ treatment in vitro. Finally, when DhL treated P. aeruginosa was co cultured with mouse macrophage J774-Eclone 
cells, the typical $P$. aeruginosa induced cellular apoptosis was found to be successfully controlled, thus indicating inhibition of virulence factors specifically of T3SS, which otherwise leads to cell toxicity and apoptosis. Although the exact mechanism of DhL mediated suppression of $P$. aeruginos $a$ virulence is still under investigation, some new data (Dinh and Barbieri, un published) highlights the possibility of DhL acting as antibacterial topo-isomerase (Kathiravan, Khilare et al. 2013). Due to high mutation rate in bacterial genome, $P$. aeruginosa is extremely difficult to eradicate from mucoid colonies (Mathee, von Schirnding et al. 2002). Therefore, as an alternative strategy, scientists are aiming at antivirulence therapy to prevent $P$. aeruginosa invasion (Clatworthy, Pierson et al. 2007; Adonizio, Kong et al. 2008; Song, Kong et al. 2010). Given the popularity of $\mathrm{DhL}$ as an antimicrobial agent, further in vivo analysis in animal models carrying $P$. aeruginosa infection may advance its potential as a successful antiPseudomonas agent. 


\subsection{Figures and legends}

\begin{tabular}{|l|l|l|l|l|}
\hline & & & & \\
\hline $\begin{array}{l}P . \quad \text { aeruginosa } \\
\text { strain }\end{array}$ & $\begin{array}{l}\text { Antimicrobial } \\
\text { agent }\end{array}$ & $\begin{array}{l}\text { MIC } \\
(\mathbf{m g} / \mathbf{m l})\end{array}$ & $\mathbf{M I C}_{90}(\mathrm{mg} / \mathrm{ml})$ & $\begin{array}{l}\mathbf{M I C}_{50} \\
(\mathbf{m g} / \mathbf{m l})\end{array}$ \\
\hline & & & & \\
\hline PAO1 & DhL & 0.48 & 0.24 & 0.12 \\
\hline & Gentamycine & 0.075 & $0.064^{*}$ & $0.032^{*}$ \\
\hline & & & & 0.24 \\
\hline PA14 & DhL & 0.96 & 0.48 & $>0.032^{*}$ \\
\hline & Gentamycine & 0.1 & $>0.064^{*}$ & \\
\hline & & & & 0.12 \\
\hline PA103 & DhL & 0.48 & 0.24 & $0.032^{*}$ \\
\hline & Gentamycine & 0.075 & $0.064^{*}$ &
\end{tabular}

Table 1: Table showing Minimum inhibitory concentration of $\mathrm{DhL}$ against $P$. aeruginosa strains showing complete killing (MIC), 50\% of killing $\left(\mathrm{MIC}_{50}\right)$ and $90 \%$ of killing $\left(\mathrm{MIC}_{90}\right) .{ }^{*}$ (Visalli, Jacobs et al. 1998). 


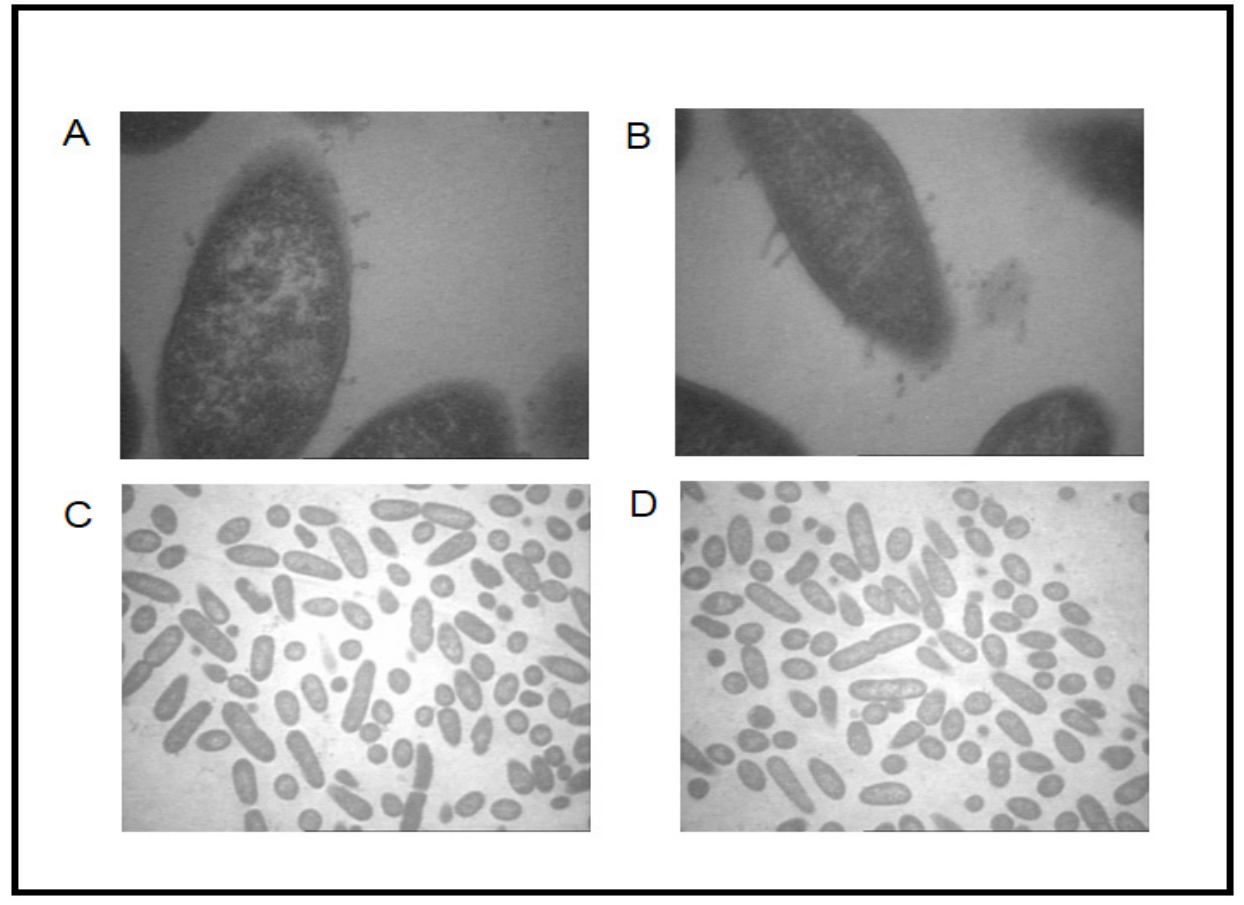

Figure 29: Electron microscopy analysis of DhL treated $\boldsymbol{P}$. aeruginosa.

(A) DhL treated $P$. aeruginosa under $20000 \mathrm{x}$ magnification showing intact cell membrane. (B) DhL treated P. aeruginosa cells without DhL treatment under $20000 \mathrm{x}$ magnifications. (C) DhL treated $P$. aeruginosa under $3000 \mathrm{x}$ magnification also showed no sign of cellular degradation. (D) $P$. aeruginosa cells under $3000 \mathrm{x}$ magnification. 


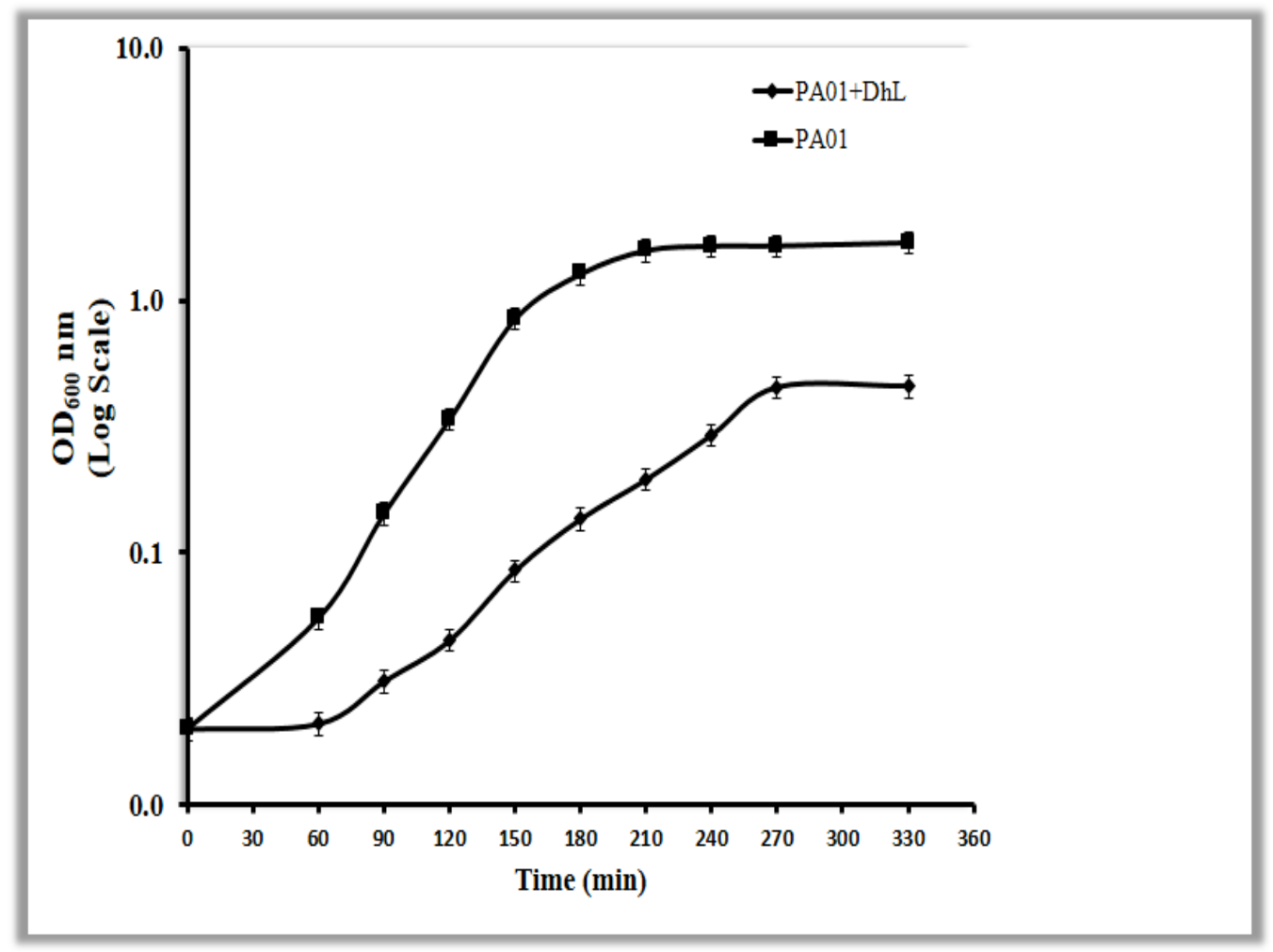

Figure 30: Influence of DhL on growth of $P$. aeruginosa.

The prototypic PAO1 was grown in the absence (*) and presence $(\bullet)$ of $\mathrm{DhL}\left(\mathrm{MIC}_{50}\right.$ $0.12 \mathrm{mg} / \mathrm{ml})$ from early $\log$ phase to stationary phase $(\sim 1.7)$ at $37^{\circ} \mathrm{C}$ with constant shaking. Generation time (between 1 to 3 hours) of PAO1 in presence of DhL (calculated from three independent experiments) is $50 \pm 0.5$ minutes while for PAO1 grown in absence of DhL is $23 \pm 0.5 \mathrm{~min}$. At least $50 \pm 5 \%$ of slowing down of generation time in the mid log phase was observed when treated with $\mathrm{DhL}\left(\mathrm{MIC}_{50}\right)$. Data represent the mean of three independent experiments \pm SEM. 

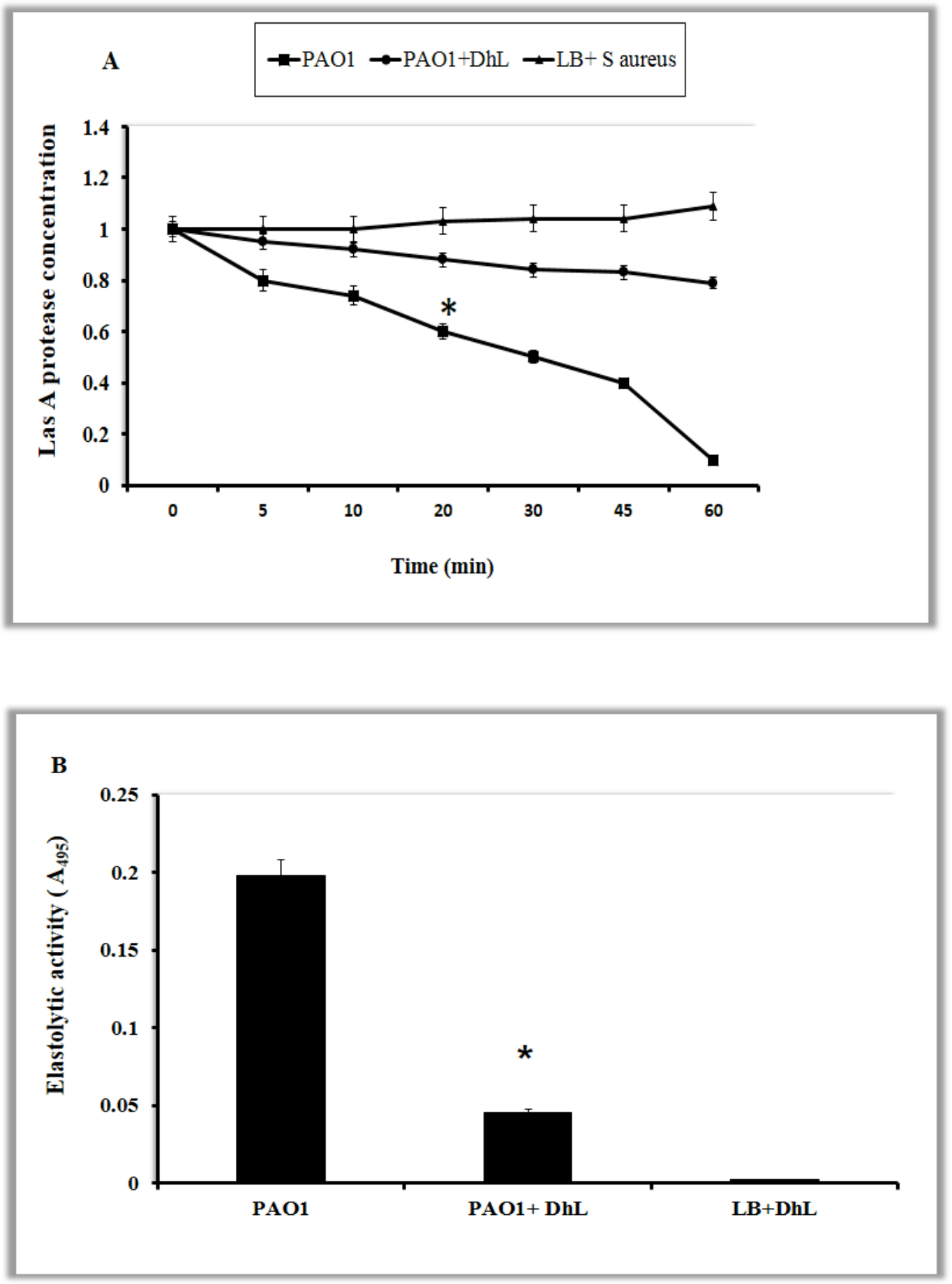

Figure 31: Effect of DhL on $P$. aeruginosa strain PAO1 protease activities.

The staphylolytic LasA (A) and elastolytic LasB (B) activities were monitored in the absence or presence of DhL $(0.12 \mathrm{mg} / \mathrm{ml})$. (A) Staphylolytic assay for the rate of LasA proteolytic activity within the supernatant fraction of the growth culture was estimated by measuring the lysis of heat inactivated intact $S$. aureus at $\mathrm{OD}_{595}$ (see Material and Methods). LB+ DhL was used as a negative control. Proteolytic activity of PAO1 
supernatant was significantly (asterisk indicated $P<0.05$ ) inhibited when grown with DhL. Data represent the mean of three independent experiments \pm SEM. (B) Levels of LasB elastolytic activity $\left(\mathrm{A}_{495}\right)$ within the PAO1 cultural supernatant with or without DhL treatment was measured by Elastin Congo red assay (Material and Method).

Bacteria were grown in LB broth at $37^{\circ} \mathrm{C}$ for 16 hours. The cultures were adjusted to an $\mathrm{OD}_{540}$ of 3.5-4.0 before harvesting to eliminate growth-related variations in elastolytic activity. DhL added LB was used as a negative control. Significant (asterisk indicated $P<0.05)$ decrease in LasB activity was observed in PAO1 when treated with DhL. Data represent the mean of three independent experiments \pm SEM. 


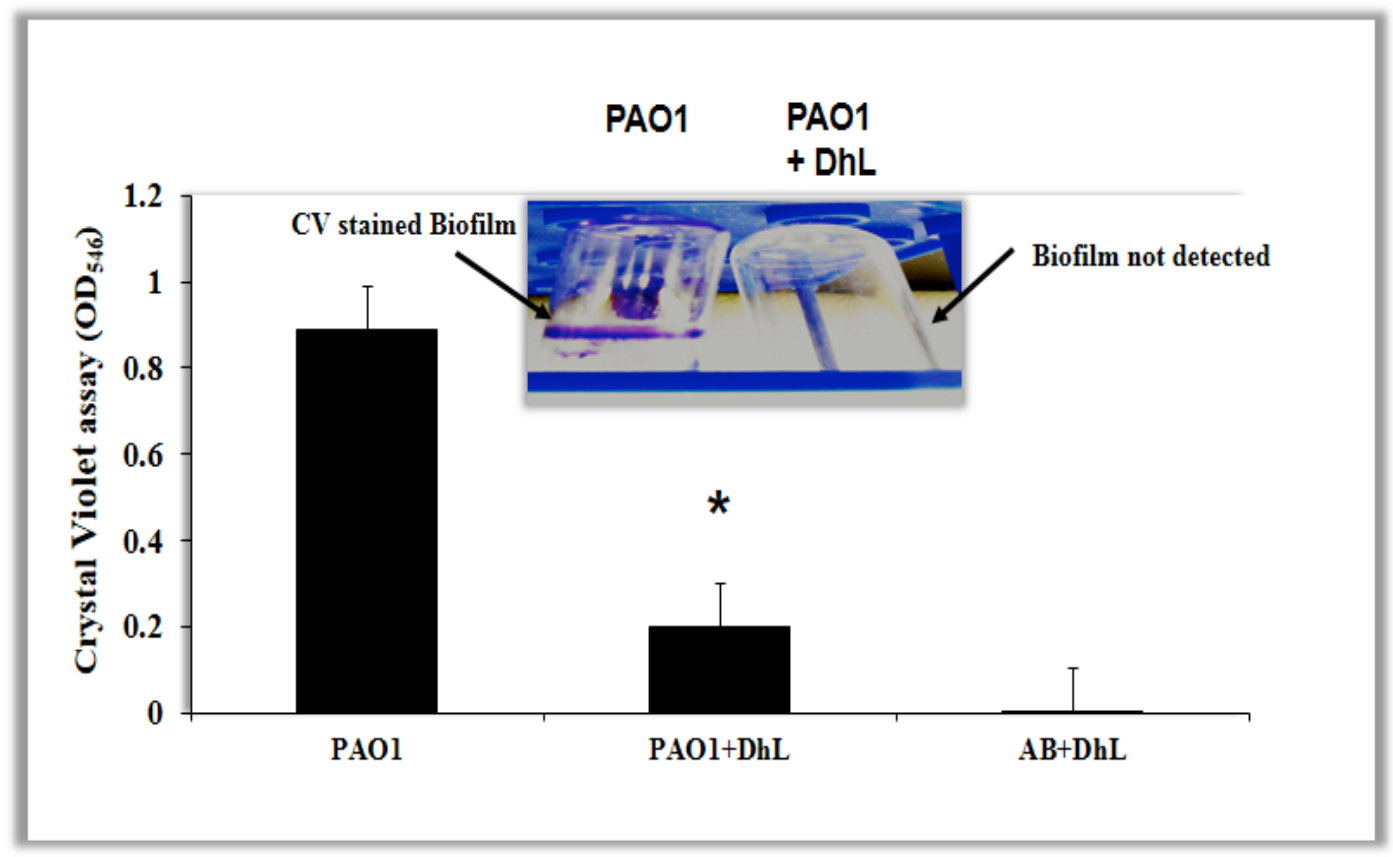

Figure 32: Effect of DhL on $\boldsymbol{P}$. aeruginosa biofilm formation.

Overnight cultures of $\mathrm{PAO} 1$ were resuspended in fresh $\mathrm{AB}$ medium in the presence and the absence DhL in PVC tubes. After 24 hours of incubation at $30^{\circ} \mathrm{C}$, the biofilms on the wall of the tubes were visualized by staining with a crystal violet solution. Biofilm formation was quantified by measuring $A_{546}$ of crystal violet-stained wells rinsed with ethanol. Each column is the mean of three individual experiments with two replicates per treatment. Inset: significant amount of crystal violet stained biofilm appeared on the testube wall carrying PAO1, but the same was absent from the testube carrying $\mathrm{DhL}$ treated PAO1. Significant (asterisk indicated $P<0.05$ ) decrease in biofilm formation was observed in PAO1 when treated with DhL. Data represent the mean of three independent experiments \pm SEM. 


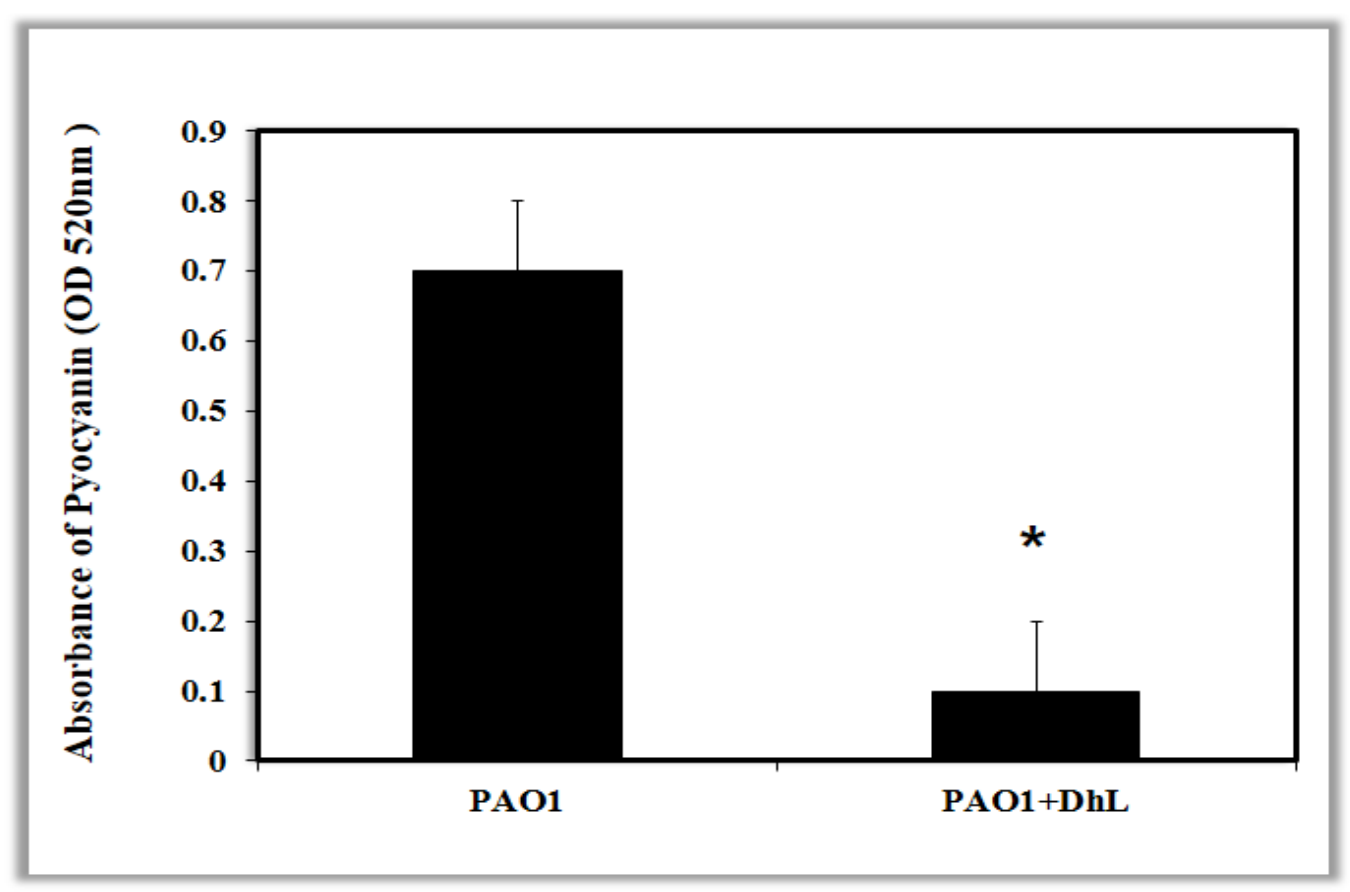

Figure 33: Pyocyanin production in DhL treated $P$. aeruginosa

Cells were grown in $1.5 \mathrm{ml}$ of static LB broth with or without DhL ( $\left.\mathrm{MIC}_{50}, 0.12 \mathrm{mg} / \mathrm{ml}\right)$ and culture was maintained at $37^{\circ} \mathrm{C}$ for 24 hours. Finally the supernatant fractions were separated by centrifugation at $14000 \mathrm{rpm}$ for 5 minutes and Pyocyanin production was determined by the chloroform: acid extraction procedure (Material and Method). Pyocyanin formation was measured at OD $520 \mathrm{~nm}$ and was compared between PAO1 culture grown in LB with or without DhL. Each column is the mean of three individual experiments with two replicates per treatment. Significant (asterisk indicated $P<0.05$ ) decrease in Pyocyanin formation in PAO1 when treated with DhL was observed. Data represent the mean of three independent experiments \pm SEM. 


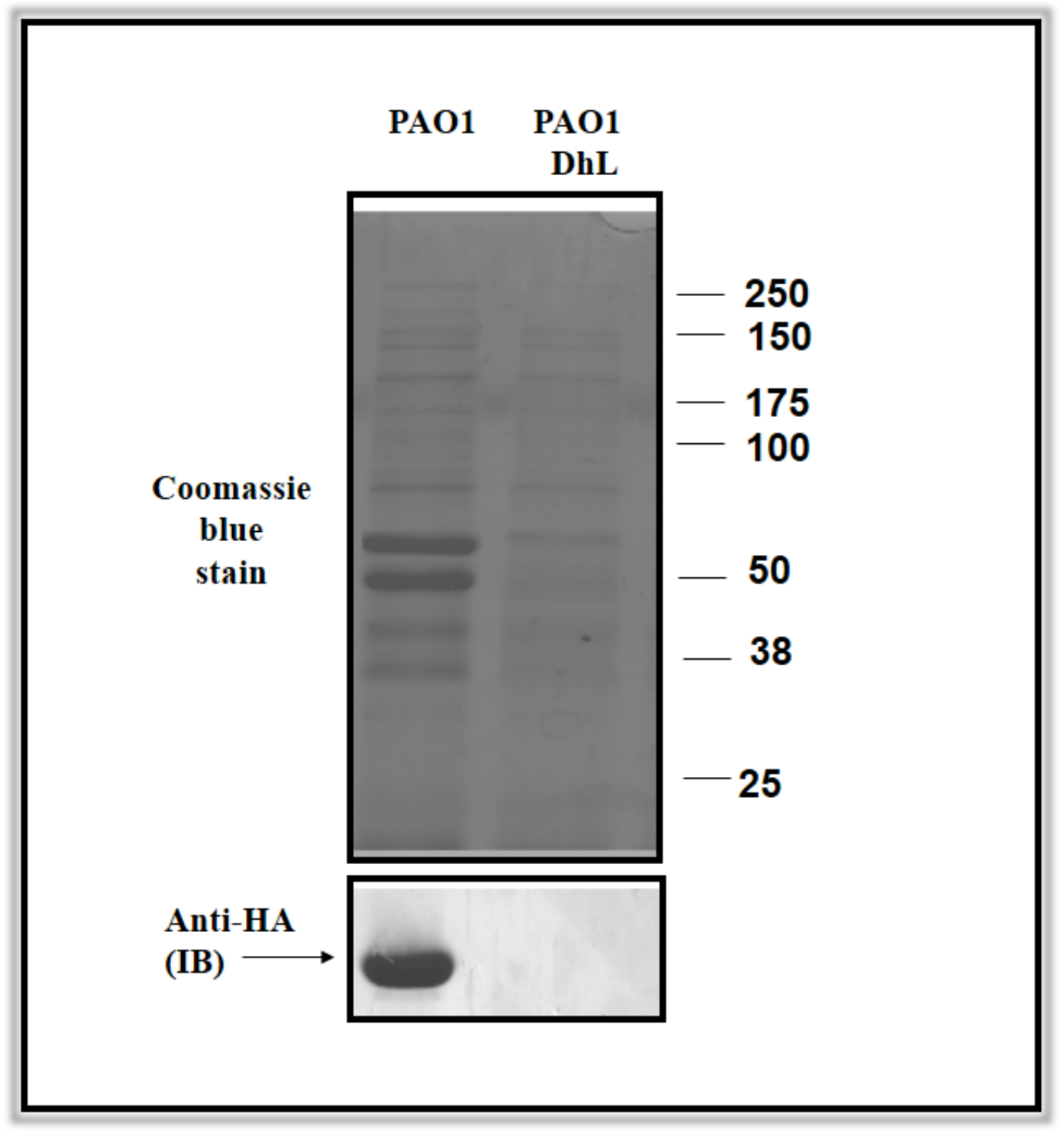

Figure 34: Effect of DhL on TypeIII secretion.

(Top Panel) Type III secretion profile of $P$. aeruginosa strains PAO1 in presence of DhL as observed with SDS-PAGE stained in coomassie blue stain. Lane 1 represents untreated PAO1 secretion profile and lane 2 represents treated PAO1 secretion profile. Under coomassie blue stain, protein bands were observed at $\sim 53 \mathrm{Kda}, \sim 49 \mathrm{Kda}$, and $\sim 37 \mathrm{Kda}$ indicating ExoT, ExoS and ExoY respectively in PAO1 Type III secretion assay, which

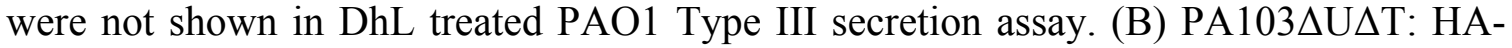
ExoS strain carrying hemaglutanin (HA) tagged ExoS was also subjected to DhL treatment and T3S profile was analyzed as described in Material and Methods. ExoS was detected in the secretion profile of untreated strain and was absent from the treated strain. HA specific antibody was used in immunoblot (IB) to confirm the presence of $\sim 49 \mathrm{Kda}$ ExoS secretion in : HA-ExoS strain as elucidated at the bottom panel. 


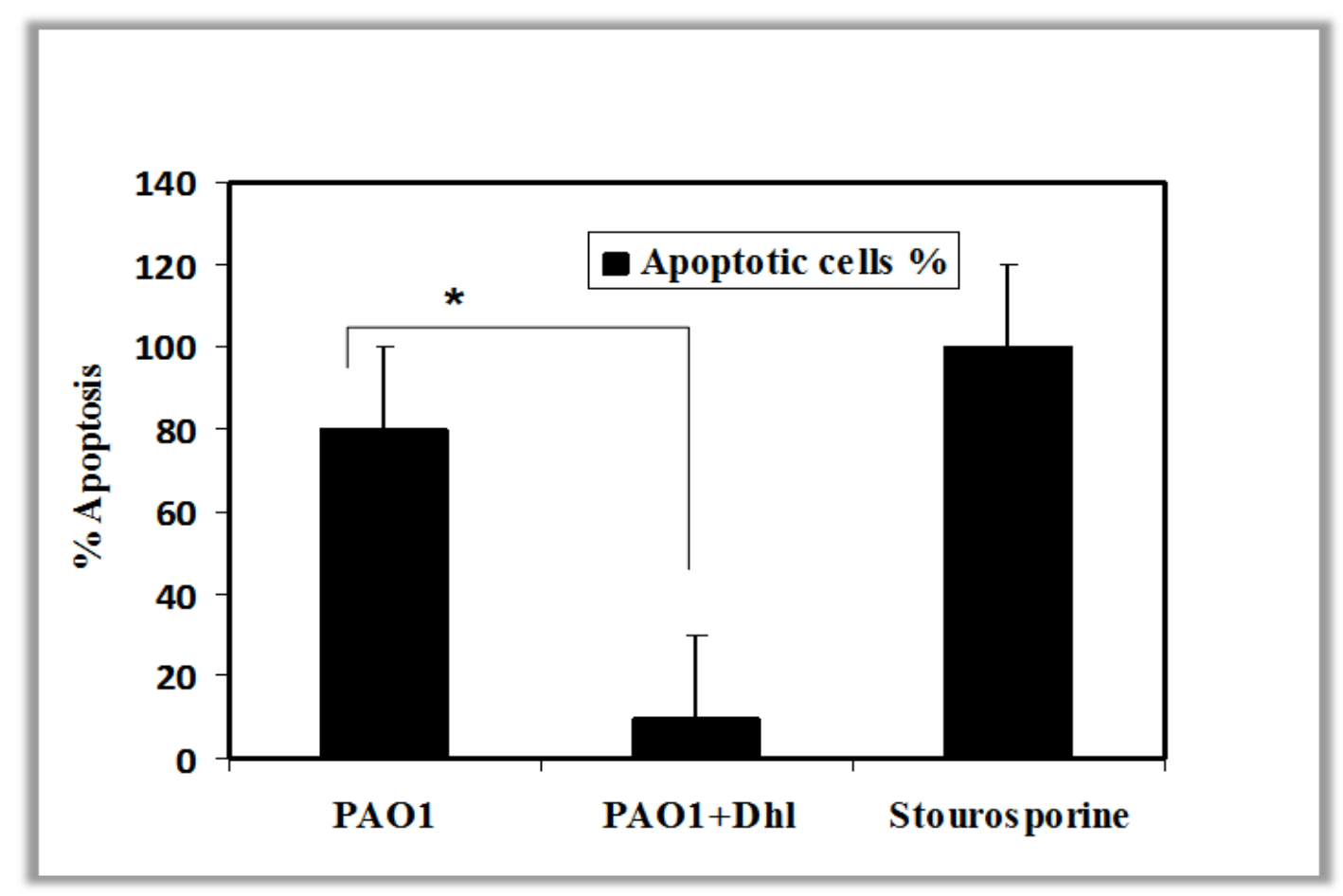

Figure 35: Effect of DhL on $P$. aeruginosa induced apoptosis.

J774-Eclone cells were plated in monolayer $\left(\sim 5 \times 10^{5}\right.$ cells per well $)$, once adhered they were infected with PAO1 bacteria previously grown in $\mathrm{LB}-\mathrm{DhL}\left(\mathrm{MIC}_{50}, 0.12 \mathrm{mg} / \mathrm{ml}\right.$ )or only LB, at a multiplicity of infection of 20 , and incubated for 2 hours at $37^{\circ} \mathrm{C}$ in a $5 \%$ $\mathrm{CO}_{2}$ incubator. In a parallel experiment equal count of J774-Eclone cells were subjected to Stourosporine (Material and Methods) for positive control. Cells were then washed with PBS, stained with Hoechst dye, and subjected to fluorescence microscopy (Material and Methods). Five fields were randomly sampled from each experimental population, and all of the cells stained with Hoechst dye in each field were counted up to 500 in total. The total number of apoptotic cells with condensed or fragmented nuclei was determined in the five sampled regions and was expressed as follows: percentage of apoptosis per sample $=$ number of apoptotic cells/total number of cells $\times 100$. Each column is the mean of three individual experiments with two replicates per treatment. Significant (asterisk indicated $P<0.05$ ) decrease in apoptotic cells were noticed when infected with PAO1 grown in presence of DhL. Data represent the mean of three independent experiments \pm SEM. 


\subsection{Reference}

Adonizio, A., K. F. Kong and K. Mathee (2008). "Inhibition of quorum sensingcontrolled virulence factor production in Pseudomonas aeruginosa by South Florida plant extracts." Antimicrob Agents Chemother 52(1): 198-203.

Ahameethunisa, A. R. and W. Hopper (2010). "Antibacterial activity of Artemisia nilagirica leaf extracts against clinical and phytopathogenic bacteria." BMC Complement Altern Med 10: 6.

Alicia B. Penissi, O. S. G., Jorge A. Guzmánc, María I. Rudolphd, Ramón S. Piezzia (2006). "Chemical and Pharmacological Properties of Dehydroleucodine, A Lactone isolated from Artemisia douglasiana Besser." Molecular Medicinal Chemistry 10: 1-11.

Ariza Espinar, L., Bonzan, N., (1992). "El matico de la región de Cuyo (Argentina)." ActaFarmaceutica Bonaerense 11: 139-145.

Bainbridge, T. and R. B. Fick, Jr. (1989). "Functional importance of cystic fibrosis immunoglobulin $\mathrm{G}$ fragments generated by Pseudomonas aeruginosa elastase." J Lab Clin Med 114(6): 728-733.

Barrera, P. A., V. Jimenez-Ortiz, C. Tonn, O. Giordano, N. Galanti and M. A. Sosa (2008). "Natural sesquiterpene lactones are active against Leishmania mexicana." J Parasitol 94(5): 1143-1149.

Benli, M., I. Kaya and N. Yigit (2007). "Screening antimicrobial activity of various extracts of Artemisia dracunculus L." Cell Biochem Funct 25(6): 681-686.

Bever, R. A. and B. H. Iglewski (1988). "Molecular characterization and nucleotide sequence of the Pseudomonas aeruginosa elastase structural gene." J Bacteriol 170(9): 4309-4314.

Bhakuni, R. S., Jain, D.C.,Sharma,R.P.,Kumar,S. (2001). "Secondray metabolites of Artemisia annua and their biological activity." Current Science 80(1): 14.

Bjorn, M. J., P. A. Sokol and B. H. Iglewski (1979). "Influence of iron on yields of extracellular products in Pseudomonas aeruginosa cultures." J Bacteriol 138(1): 193-200.

Bohlmann, F. and C. Zdero (1972). "[Polyacetylenic compounds, 205. On the constituents of the tribe Arctotideae]." Chem Ber 105(4): 1245-1257.

Brengio, S. D., S. A. Belmonte, E. Guerreiro, O. S. Giordano, E. O. Pietrobon and M. A. Sosa (2000). "The sesquiterpene lactone dehydroleucodine $(\mathrm{DhL})$ affects the growth of cultured epimastigotes of Trypanosoma cruzi." J Parasitol 86(2): 407-412.

Buret, A. and A. W. Cripps (1993). "The immunoevasive activities of Pseudomonas aeruginosa. Relevance for cystic fibrosis." Am Rev Respir Dis 148(3): 793-805. 
Choi, E., H. Park, J. Lee and G. Kim (2013). "Anticancer, antiobesity, and antiinflammatory activity of Artemisia species in vitro." J Tradit Chin Med 33(1): 92-97.

Clatworthy, A. E., E. Pierson and D. T. Hung (2007). "Targeting virulence: a new paradigm for antimicrobial therapy." Nat Chem Biol 3(9): 541-548.

Costerton, J. W., Z. Lewandowski, D. E. Caldwell, D. R. Korber and H. M. Lappin-Scott (1995). "Microbial biofilms." Annu Rev Microbiol 49: 711-745.

Dasgupta, M. K. (1996). "Biofilm causes decreased production of interferon-gamma." J Am Soc Nephrol 7(6): 877-882.

Davies, D. (2003). "Understanding biofilm resistance to antibacterial agents." Nat Rev Drug Discov 2(2): 114-122.

Engler, K., K. Muhlemann, C. Garzoni, H. Pfahler, T. Geiser and C. von Garnier (2012). "Colonisation with Pseudomonas aeruginosa and antibiotic resistance patterns in COPD patients." Swiss Med Wkly 142: 0.

Fleiszig, S. M., T. S. Zaidi, M. J. Preston, M. Grout, D. J. Evans and G. B. Pier (1996). "Relationship between cytotoxicity and corneal epithelial cell invasion by clinical isolates of Pseudomonas aeruginosa." Infect Immun 64(6): 2288-2294.

Giordano, O. S., E. Guerreiro, M. J. Pestchanker, J. Guzman, D. Pastor and T. Guardia (1990). "The gastric cytoprotective effect of several sesquiterpene lactones." J Nat Prod 53(4): 803-809.

Gomes, M. Z., C. R. Machado, S. da Conceicao Mde, J. A. Ortega, S. M. Neves, M. C. Lourenco and M. D. Asensi (2011). "Outbreaks, persistence, and high mortality rates of multiresistant Pseudomonas aeruginosa infections in a hospital with AIDS-predominant admissions." Braz J Infect Dis 15(4): 312-322.

Grossowicz, N., P. Hayat and Y. S. Halpern (1957). "Pyocyanine biosynthesis by Pseudomonas aeruginosa." J Gen Microbiol 16(3): 576-583.

Guardia, T., J. A. Guzman, M. J. Pestchanker, E. Guerreiro and O. S. Giordano (1994). "Mucus synthesis and sulfhydryl groups in cytoprotection mediated by dehydroleucodine, a sesquiterpene lactone." J Nat Prod 57(4): 507-509.

Hamdaoui, A., F. Wund-Bisseret and J. G. Bieth (1987). "Fast solubilization of human lung elastin by Pseudomonas aeruginosa elastase." Am Rev Respir Dis 135(4): 860-863.

Hancock, R. E. and D. P. Speert (2000). "Antibiotic resistance in Pseudomonas aeruginosa: mechanisms and impact on treatment." Drug Resist Updat 3(4): 247-255.

Hauser, A. R. (2009). "The type III secretion system of Pseudomonas aeruginosa: infection by injection." Nat Rev Microbiol 7(9): 654-665. 
Hauser, A. R. and J. N. Engel (1999). "Pseudomonas aeruginosa induces type-IIIsecretion-mediated apoptosis of macrophages and epithelial cells." Infect Immun 67(10): 5530-5537.

Hauser, A. R. and P. Sriram (2005). "Severe Pseudomonas aeruginosa infections. Tackling the conundrum of drug resistance." Postgrad Med 117(1): 41-48.

Heck, L. W., P. G. Alarcon, R. M. Kulhavy, K. Morihara, M. W. Russell and J. F. Mestecky (1990). "Degradation of IgA proteins by Pseudomonas aeruginosa elastase." J Immunol 144(6): 2253-2257.

Heck, L. W., K. Morihara, W. B. McRae and E. J. Miller (1986). "Specific cleavage of human type III and IV collagens by Pseudomonas aeruginosa elastase." Infect Immun 51(1): 115-118.

Hentzer, M., H. Wu, J. B. Andersen, K. Riedel, T. B. Rasmussen, N. Bagge, N. Kumar, M. A. Schembri, Z. Song, P. Kristoffersen, M. Manefield, J. W. Costerton, S. Molin, L. Eberl, P. Steinberg, S. Kjelleberg, N. Hoiby and M. Givskov (2003). "Attenuation of Pseudomonas aeruginosa virulence by quorum sensing inhibitors." EMBO J 22(15): 3803-3815.

Hogardt, M. and J. Heesemann (2013). "Microevolution of Pseudomonas aeruginosa to a Chronic Pathogen of the Cystic Fibrosis Lung." Curr Top Microbiol Immunol 358: 91118.

Hoge, R., Pelzer, A., Rosenau, F., Wilhelm, S. (2010). "'weapons of a pathogen: Proteases and their role in virulence of Pseudomonas aeruginosa." Current reseach, Technology and education topics in applied microbiology and microbial technology 1: 383-395

Holder, I. A., A. N. Neely and D. W. Frank (2001). "Type III secretion/intoxication system important in virulence of Pseudomonas aeruginosa infections in burns." Burns 27(2): 129-130.

Holloway, B. W. and A. F. Morgan (1986). "Genome organization in Pseudomonas." Annu Rev Microbiol 40: 79-105.

Jia, J., Y. Wang, L. Zhou and S. Jin (2006). "Expression of Pseudomonas aeruginosa toxin ExoS effectively induces apoptosis in host cells." Infect Immun 74(12): 6557-6570.

Kalemba, D., D. Kusewicz and K. Swiader (2002). "Antimicrobial properties of the essential oil of Artemisia asiatica Nakai." Phytother Res 16(3): 288-291.

Kathiravan, M. K., M. M. Khilare, K. Nikoomanesh, A. S. Chothe and K. S. Jain (2013). "Topoisomerase as target for antibacterial and anticancer drug discovery." J Enzyme Inhib Med Chem 28(3): 419-435. 
Kaufman, M. R., J. Jia, L. Zeng, U. Ha, M. Chow and S. Jin (2000). "Pseudomonas aeruginosa mediated apoptosis requires the ADP-ribosylating activity of exoS." Microbiology 146 ( Pt 10): 2531-2541.

Kessler, E. and M. Safrin (1988). "Synthesis, processing, and transport of Pseudomonas aeruginosa elastase." J Bacteriol 170(11): 5241-5247.

Kessler, E., M. Safrin, W. R. Abrams, J. Rosenbloom and D. E. Ohman (1997). "Inhibitors and specificity of Pseudomonas aeruginosa LasA." J Biol Chem 272(15): 9884-9889.

Kessler, E., M. Safrin, J. C. Olson and D. E. Ohman (1993). "Secreted LasA of Pseudomonas aeruginosa is a staphylolytic protease." J Biol Chem 268(10): 7503-7508.

Kim, J. H., S. H. Jung, Y. I. Yang, J. H. Ahn, J. G. Cho, K. T. Lee, N. I. Baek and J. H. Choi (2013). "Artemisia leaf extract induces apoptosis in human endometriotic cells through regulation of the p38 and NFkappaB pathways." J Ethnopharmacol 145(3): 767775 .

Krcmery, V., J. Koprnova, M. Gogova, E. Grey and J. Korcova (2006). "Pseudomonas aeruginosa bacteraemia in cancer patients." J Infect 52(6): 461-463.

Kudoh, I., J. P. Wiener-Kronish, S. Hashimoto, J. F. Pittet and D. Frank (1994). "Exoproduct secretions of Pseudomonas aeruginosa strains influence severity of alveolar epithelial injury." Am J Physiol 267(5 Pt 1): L551-556.

Kukavica-Ibrulj, I., A. Bragonzi, M. Paroni, C. Winstanley, F. Sanschagrin, G. A. O'Toole and R. C. Levesque (2008). "In vivo growth of Pseudomonas aeruginosa strains PAO1 and PA14 and the hypervirulent strain LESB58 in a rat model of chronic lung infection." J Bacteriol 190(8): 2804-2813.

Lee, V. T., R. S. Smith, B. Tummler and S. Lory (2005). "Activities of Pseudomonas aeruginosa effectors secreted by the Type III secretion system in vitro and during infection." Infect Immun 73(3): 1695-1705.

Livermore, D. M. (1984). "Penicillin-binding proteins, porins and outer-membrane permeability of carbenicillin-resistant and -susceptible strains of Pseudomonas aeruginosa." J Med Microbiol 18(2): 261-270.

Livermore, D. M. (2001). "Of Pseudomonas, porins, pumps and carbapenems." J Antimicrob Chemother 47(3): 247-250.

Lyczak, J. B., C. L. Cannon and G. B. Pier (2002). "Lung infections associated with cystic fibrosis." Clin Microbiol Rev 15(2): 194-222. 
Mahajan-Miklos, S., L. G. Rahme and F. M. Ausubel (2000). "Elucidating the molecular mechanisms of bacterial virulence using non-mammalian hosts." Mol Microbiol 37(5): 981-988.

Mahboubi, M. and N. Kazempour (2009). "The Antimicrobial Activity of Essential Oil from Perovskia abrotanoides Karel and its Main Components." Indian J Pharm Sci 71(3): 343-347.

Mathee, A., Y. E. von Schirnding, J. Levin, A. Ismail, R. Huntley and A. Cantrell (2002). "A survey of blood lead levels among young Johannesburg school children." Environ Res 90(3): 181-184.

McIver, K. S., E. Kessler and D. E. Ohman (2004). "Identification of residues in the Pseudomonas aeruginosa elastase propeptide required for chaperone and secretion activities." Microbiology 150(Pt 12): 3969-3977.

Mesaros, N., P. Nordmann, P. Plesiat, M. Roussel-Delvallez, J. Van Eldere, Y. Glupczynski, Y. Van Laethem, F. Jacobs, P. Lebecque, A. Malfroot, P. M. Tulkens and F. Van Bambeke (2007). "Pseudomonas aeruginosa: resistance and therapeutic options at the turn of the new millennium." Clin Microbiol Infect 13(6): 560-578.

Micek, S. T., A. E. Lloyd, D. J. Ritchie, R. M. Reichley, V. J. Fraser and M. H. Kollef (2005). "Pseudomonas aeruginosa bloodstream infection: importance of appropriate initial antimicrobial treatment." Antimicrob Agents Chemother 49(4): 1306-1311.

Mollenhauer, H. H., D. J. Morre and B. S. Hass (1977). "Plasma membrane transformations in spermatogenesis revealed by aldehyde fixatives containing tannic acid." J Ultrastruct Res 61(2): 166-171.

Moss, J., M. E. Ehrmantraut, B. D. Banwart, D. W. Frank and J. T. Barbieri (2001). "Sera from adult patients with cystic fibrosis contain antibodies to Pseudomonas aeruginosa type III apparatus." Infect Immun 69(2): 1185-1188.

Nordmann, P. and M. Guibert (1998). "Extended-spectrum beta-lactamases in Pseudomonas aeruginosa." J Antimicrob Chemother 42(2): 128-131.

O'Toole, G. A. and R. Kolter (1998). "Initiation of biofilm formation in Pseudomonas fluorescens WCS365 proceeds via multiple, convergent signalling pathways: a genetic analysis." Mol Microbiol 28(3): 449-461.

Pedersen, S. S. (1992). "Lung infection with alginate-producing, mucoid Pseudomonas aeruginosa in cystic fibrosis." APMIS Suppl 28: 1-79.

Pesci, E. C., J. B. Milbank, J. P. Pearson, S. McKnight, A. S. Kende, E. P. Greenberg and B. H. Iglewski (1999). "Quinolone signaling in the cell-to-cell communication system of Pseudomonas aeruginosa." Proc Natl Acad Sci U S A 96(20): 11229-11234. 
Priestap, H. A., K. A. Abboud, A. E. Velandia, L. A. Lopez and M. A. Barbieri (2011). "Dehydro-leucodin: a guaiane-type sesquiterpene lactone." Acta Crystallogr Sect E Struct Rep Online 67(Pt 12): o3470.

Rahme, L. G., E. J. Stevens, S. F. Wolfort, J. Shao, R. G. Tompkins and F. M. Ausubel (1995). "Common virulence factors for bacterial pathogenicity in plants and animals." Science 268(5219): 1899-1902.

Ramezani, M., B. S. Fazli-Bazzaz, F. Saghafi-Khadem and A. Dabaghian (2004). "Antimicrobial activity of four Artemisia species of Iran." Fitoterapia 75(2): 201-203.

Reyes, E. A., M. J. Bale, W. H. Cannon and J. M. Matsen (1981). "Identification of Pseudomonas aeruginosa by pyocyanin production on Tech agar." J Clin Microbiol 13(3): 456-458.

Rubin Grandis, J., B. F. t. Branstetter and V. L. Yu (2004). "The changing face of malignant (necrotising) external otitis: clinical, radiological, and anatomic correlations." Lancet Infect Dis 4(1): 34-39.

Saulnier, J. M., F. M. Curtil, M. C. Duclos and J. M. Wallach (1989). "Elastolytic activity of Pseudomonas aeruginosa elastase." Biochim Biophys Acta 995(3): 285-290.

Sawa, T., M. Ohara, K. Kurahashi, S. S. Twining, D. W. Frank, D. B. Doroques, T. Long, M. A. Gropper and J. P. Wiener-Kronish (1998). "In vitro cellular toxicity predicts Pseudomonas aeruginosa virulence in lung infections." Infect Immun 66(7): 3242-3249.

Schultz, D. R. and K. D. Miller (1974). "Elastase of Pseudomonas aeruginosa: inactivation of complement components and complement-derived chemotactic and phagocytic factors." Infect Immun 10(1): 128-135.

Setzer, W. N., B. Vogler, J. M. Schmidt, J. G. Leahy and R. Rives (2004). "Antimicrobial activity of Artemisia douglasiana leaf essential oil." Fitoterapia 75(2): 192-200.

Smith, R. S. and B. H. Iglewski (2003). "Pseudomonas aeruginosa quorum sensing as a potential antimicrobial target." J Clin Invest 112(10): 1460-1465.

Song, Z., K. F. Kong, H. Wu, N. Maricic, B. Ramalingam, H. Priestap, L. Schneper, J. M. Quirke, N. Hoiby and K. Mathee (2010). "Panax ginseng has anti-infective activity against opportunistic pathogen Pseudomonas aeruginosa by inhibiting quorum sensing, a bacterial communication process critical for establishing infection." Phytomedicine 17(13): 1040-1046.

Stover, C. K., X. Q. Pham, A. L. Erwin, S. D. Mizoguchi, P. Warrener, M. J. Hickey, F. S. Brinkman, W. O. Hufnagle, D. J. Kowalik, M. Lagrou, R. L. Garber, L. Goltry, E. Tolentino, S. Westbrock-Wadman, Y. Yuan, L. L. Brody, S. N. Coulter, K. R. Folger, A. Kas, K. Larbig, R. Lim, K. Smith, D. Spencer, G. K. Wong, Z. Wu, I. T. Paulsen, J. Reizer, M. H. Saier, R. E. Hancock, S. Lory and M. V. Olson (2000). "Complete genome 
sequence of Pseudomonas aeruginosa PAO1, an opportunistic pathogen." Nature 406(6799): 959-964.

Toder, D. S., M. J. Gambello and B. H. Iglewski (1991). "Pseudomonas aeruginosa LasA: a second elastase under the transcriptional control of lasR." Mol Microbiol 5(8): 2003-2010.

Usher, L. R., R. A. Lawson, I. Geary, C. J. Taylor, C. D. Bingle, G. W. Taylor and M. K. Whyte (2002). "Induction of neutrophil apoptosis by the Pseudomonas aeruginosa exotoxin pyocyanin: a potential mechanism of persistent infection." J Immunol 168(4): 1861-1868.

Vega, A. E., G. H. Wendel, A. O. Maria and L. Pelzer (2009). "Antimicrobial activity of Artemisia douglasiana and dehydroleucodine against Helicobacter pylori." J Ethnopharmacol 124(3): 653-655.

Visalli, M. A., M. R. Jacobs and P. C. Appelbaum (1998). "Determination of activities of levofloxacin, alone and combined with gentamicin, ceftazidime, cefpirome, and meropenem, against 124 strains of Pseudomonas aeruginosa by checkerboard and timekill methodology." Antimicrob Agents Chemother 42(4): 953-955.

Voynow, J. A., B. M. Fischer and S. Zheng (2008). "Proteases and cystic fibrosis." Int J Biochem Cell Biol 40(6-7): 1238-1245.

Weinstein, M. P., M. L. Towns, S. M. Quartey, S. Mirrett, L. G. Reimer, G. Parmigiani and L. B. Reller (1997). "The clinical significance of positive blood cultures in the 1990s: a prospective comprehensive evaluation of the microbiology, epidemiology, and outcome of bacteremia and fungemia in adults." Clin Infect Dis 24(4): 584-602.

Wendel, G. H., A. O. Maria, J. A. Guzman, O. Giordano and L. E. Pelzer (2008). "Antidiarrheal activity of dehydroleucodine isolated from Artemisia douglasiana." Fitoterapia 79(1): 1-5.

Wendel, G. H., A. O. Maria, F. Mohamed, S. Dominguez, L. Scardapane, O. S. Giordano, E. Guerreiro and J. A. Guzman (1999). "Effect of dehydroleucodine in experimental colitis in rats and mice." Pharmacol Res 40(4): 339-344.

Wisplinghoff, H., H. Seifert, S. M. Tallent, T. Bischoff, R. P. Wenzel and M. B. Edmond (2003). "Nosocomial bloodstream infections in pediatric patients in United States hospitals: epidemiology, clinical features and susceptibilities." Pediatr Infect Dis J 22(8): 686-691.

Woolfrey, B. F., J. M. Fox, R. T. Lally and C. O. Quall (1982). "Broth microdilution testing of Pseudomonas aeruginosa and aminoglycosides: need for employing dilutions differing by small arithmetic increments." J Clin Microbiol 16(4): 663-667. 


\section{Chapter 6}

General discussion and future directions 


\subsection{Discussion}

Pseudomonas aeruginosa, a pathogen with many potential virulence factors, colonizes and infects essentially any mammalian tissue. The organism possesses a multitude of factors which promote host cell adherence, host tissue damage, elicit inflammation and disrupt defense mechanisms. Pseduomonas aeruginosa infections could be severe and life threatening. Once infected, it is difficult to eliminate the pathogen because of the limited susceptibility to antimicrobial agents and the frequent occurrence of antibiotic resistance during therapy (Carmeli, Troillet et al. 1999; Lister, Wolter et al. 2009). In spite of the ubiquitous niche of this microorganism, and the frequency with which it is encountered by human population, most human hosts are effective to counteract the infectious process via their innate immune system (Lyczak, Cannon et al. 2000). However, the genetically compromised immune systems such as in the CF patients, dramatically increases host susceptibility to $P$. aeruginosa infection (Lyczak, Cannon et al. 2000). Almost all clinical cases of $P$. aeruginosa infection are associated with compromised host defenses such as AIDS, burn wood patients and patients undergoing chemotherapy (Chitkara and Feierabend 1981; Bendig, Kyle et al. 1987; Franzetti, Cernuschi et al. 1992). Radiations and chemotherapies break down the tissue of natural infection barriers such as the skin and covering of the gastrointestinal tract making it vulnerable towards bacterial and fungal infection. Because of its clinical importance, the pathogen has been subjected to extensive studies for a more detailed molecular and cellular understanding of the bacterial and host interaction. An overall comprehension of the pathogenic process of $P$. aeruginosa will be of increasing importance to the development of preventative strategies. 
More than 15 species of Gram negative human pathogens including Salmonella spp, Shigella flexneri, Yersinia spp, Escherecia coli, Chlamydia spp and Pseudomonas aeruginosa shares Type III secretion (T3S) apparatus (Hueck 1998; Pallen, Beatson et al. 2005; Keyser, Elofsson et al. 2008). Pseduomonas aeruginosa T3S effectors ExoS and ExoT target many host proteins along with Ras and Rho family of GTPases involved in host cell trafficking (Deng and Barbieri 2008). The T3S effector ExoS has also been implicated as an antiphagocytic factor along with enhancing cytotoxicity and lung injury in infected animals (Nicas, Frank et al. 1985; Apodaca, Bomsel et al. 1995; FrithzLindsten, Du et al. 1997). Macrophages are critical in protecting the lung and mucosal surfaces against infection and may ingest and kill potential pathogens in the absence of exogenous opsonins. Although phagocytic cell dysfunction has been suggested, it has not yet been demonstrated in the case of P. aeruginosa infection (Cowell, Chen et al. 2000; Celli and Finlay 2002). Therefore, with the emergence of interest on various pathogenic mechanisms adopted by $P$. aeruginosa, this dissertation ventured the molecular mechanism by which T3S system manipulates host phagocytic defense, to better understand the dynamics of phagosome-pathogen interaction.

Phagocytosis is a type of endocytosis where active Rab5 (GTP-bound Rab5) recruitment is associated with nascent phagosome. Activity of Rab5 has been correlated with enhanced antimicrobial properties in phagosomes (Duclos, Diez et al. 2000). This report demonstrated for the first time that Rab5 activity is critical in the process of internalization of $P$. aeruginosa by macrophage cells. A sharp inhibition of endogenous Rab5 activity characterized by weaker phagocytic response was observed in J774-Eclone macrophage cell line within 15 minutes (early phagocytic event) of live P. aeruginosa 
internalization. The consequences of Rab5 activity was successfully reversed during the phagocytic process by over expressing constitutively active Rab5 Q79L mutant protein in J774-Eclone macrophage cells. In the presence of Rab5 Q79L, a GTP hydrolysis defective mutant, macrophage cells showed $\sim 4$ fold increase in phagocytic index and uninterrupted Rab5 activity. Examining the plausible role of several T3S effectors in the inhibition of Rab5 activity and subsequent avoidance to phagocytic killing, different strains of $P$. aeruginosa were used in this study. Analyzing the phagocytic indices in $P$. aeruginosa strains lacking one or more of the four T3S effector, it was found that little or no phagocytic inhibition was exhibited by $\mathrm{PA} 103 \Delta \mathrm{U} \Delta \mathrm{T}$, a T3S effector less strain. Interestingly, macrophages engulfing PA103 strain (lacking ExoS and ExoY) showed 3 fold higher phagocytic index compared to cells engulfing PAO1 (carrying ExoS, ExoT, ExoY). Further analysis discarded the effect of ExoT and ExoY in Rab5 modulation leaving ExoS as an essential modulator of Rab5 activity and phagocytosis in J774-Eclone cells. Lack of ExoS increased susceptibility towards macrophage mediated killing in $P$. aeruginosa strain PA103 although cumulative effects of other T3S effectors and several virulent factors showed some antiphagocytic effect in J774-Eclone cells and never matched up with the prompt and high phagocytic indices of heat inactivated $P$. aeruginosa.

In a different investigation undertaking the inhibiting role of ExoS on Rab5 activity, $P$. aeruginosa strains carrying either of the two functional domains of ExoS were subjected to phagocytosis followed by a detailed analysis of Rab5 activity. PA103 $\Delta \mathrm{U} \Delta \mathrm{T}$ strain carrying full length ExoS protein (with both ADP-ribosylating domain (ADPr) and Rho-GTPase activating (Rho-GAP) domain) as well as PA103 $\Delta \mathrm{U} \Delta \mathrm{T}$ strain carrying only 
ExoS ADPr domain, accounted for diminished level GTP-bound active Rab5 form in J774-Eclone cells during the process of phagocytosis. On the other hand, Rho-GAP activity of ExoS showed anti-internalization effect but didn't influence Rab5 function. The data was supported by in vivo observation on Rab5 activity when ExoS full length, ExoS ADPr or ExoS Rho-GAP was expressed in macrophage cells. Formation of endogenous Rab5-GTP was significantly inhibited while expressing full length ExoS protein or ExoS ADPr domain, but not ExoS Rho-GAP domain in J774-Eclone cells. Since, the Rab5 nucleotide status was found to be integral in P. aeruginosa phagocytosis, a second approach was designed to invoke Rab5 activity in J774-Eclone macrophages. Rabex5, Rin1 and Rap6, known to act as Rab5 GTP exchange factors, were over expressed in J774-Eclone macrophages individually. Internalization of heat inactivated $P$. aeruginosa raised significantly in cells over expressing each one of Rab5-GEFs, showing maximum effect from Rin1 over expression. Each of the Rab5-GEFs also enhanced Rab5 nucleotide status although to different degrees. But, in presence of live $P$. aeruginosa, only the effect of Rin1 over expression enhanced both phagocytic activity and Rab5 nucleotide status from the negative influence, suggesting specific preferences of Rab5 towards its GEFs in phagocytosis of live $P$. aeruginosa.

Over all, the primary analysis directed importance towards Rab5 nucleotide status and selective GEF mediated Rab5 activation pathway in P. aeruginosa phagocytosis. Another interesting aspect which attracted the focus of this study was ExoS influenced modification of Rab5 protein. Rab5 has been studied as a substrate for ExoS in vitro ADP-ribosylation activity (Barbieri, Sha et al. 2001; Fraylick, Rucks et al. 2002). But the ADP-ribosylation target sites on Rab5 needed to be explored. ExoS exhibits specificity 
towards arginine sites on the substrate protein (Laing, Unger et al. 2011). In order to determine the key arginine sites in Rab5, all 11 arginines in positions 4, 8, 39, 81, 91, 110, 120,141, 195, 197 and 209 were modified to alanine and purified with His-tagged expression vector for in vitro analysis. Although individual alteration of arginine to alanine at the key sites didn't alter Rab5 GTP binding capacity, they responded equally towards ADP-ribosylation by ExoS. Thus, suggesting more than one arginine residues are targeted as ADP-ribosylation sites on Rab5. Another interesting observation was obtained from this part of the study about ADP-ribosylated Rab5 protein. In vitro GTP binding assay suggested that post ADP-ribosylation, Rab5 losses its structural conformation otherwise favorable for GTP binding. Rab5 Q79L purified protein was able to retain GTP binding capacity post ADP-ribosylation unlike Rab5 WT form further indicating that it's innate GTP binding conformation was not interrupted by the modification.

Further assuring the importance of Rab5 nucleotide status, an in vivo analysis was performed to assess the role of Rin1 in rescuing Rab5 activity during invasion of live $P$. aeruginosa. A detailed examination on endogenous Rab5 activity status through out an hour of phagocytosis of heat inactivated P. aeruginosa showed a transient flash of active Rab5 between 1-20 minutes after initiation. While at the time of live P. aeruginosa invasion, the activity of endogenous Rab5 appeared bleakly up to 5 minutes time point. Hence, it was confirmed that at a very early phase of phagocytosis, Rab5 activity is diminished due to the negative effects of $P$. aeruginosa virulence. Upon over expression of Rab5 GEF, Rin1, an intrinsic active Rab5 cycle was generated sustaining the Rab5 activity up to 20 minutes in both live and heat inactivated $P$. aeruginosa invasion. Although, this observation is redundant for phagocytosis of heat inactivated form of $P$. 
aeruginosa, it was of utmost interest in the case of disabled phagocytic response in the case of the live form. To investigate the molecular mechanism by which Rin1 generates an active cycle of Rab5, a domain wise analysis of Rin1 was executed in live $P$. aeruginosa invasion. Rin1 $\mathrm{C}$ terminal region containing Rab5 activating Vps9 domain and Ras binding RA domain attributed towards a significant up regulation of phagocytic index for live $P$. aeruginosa in contrast to Rin $1 \mathrm{~N}$ terminal region containing SH2 and Proline rich domains. Further, analyzing the individual contribution of Vps9 or RA domains showed no effect on phagocytic index suggesting a coordinated activity of both domains towards phagocytosis of live $P$. aeruginosa. The observation was re assured by analyzing the fate of the live bacteria once internalized by J774-Eclone macrophages in presence of either $\mathrm{N}$ or $\mathrm{C}$ terminal region of Rin1, when data showed low survival rate in internalized bacteria in presence of Rin $1 \mathrm{C}$ terminal region rather than Rin1 $\mathrm{N}$ terminal region. The Rin1 RA domain interplays a role between Rin1 and Ras by attributing to an interaction of Rin1 with active GTP-bound Ras as a Ras effector (Wang, Waldron et al. 2002), the observation which also corroborates with other studies suggesting the requirement for active-Ras/Rin1 interaction essential for Rab5 activity (Roberts, Barbieri et al. 2000). At this point, a simple analysis of Ras activity was performed in macrophage cell line in presence of either live or heat inactivated P. aeruginosa. Results showed in both cases, Ras activity retained transiently for a period of 1-15 minutes essentially rising to a peak around 2.5-10 minutes. When Ras and Rin1 interaction was examined in live $P$. aeruginosa phagocytosis, a positive signal was received between 2.5-20 minutes suggesting a possible role of Rin1 as Ras effector in $P$. aeruginosa engulfing macrophages. Taken together, these data suggests an active role of Ras effector Rin1 also 
aiding Rab5 as a GEF and enhancing Rab5 function during live $P$. aeruginosa phagocytosis. Over all, this molecular mechanism was able to rescue phagocytic activity in macrophages in presence of live $P$. aeruginosa. Finally, an in vitro study revealed a diminished interaction between Rab5/Ras and Rin1 when Rab5/Ras were ADPribosylated, directing attention to speculate any alteration of recognition patterns or binding patterns for ADP-ribosylated Rab5 or Ras with their respective effector proteins and GEFs. In Summery, Rin1 over expression in macrophages generated a transient intrinsic cycle of active Rab5 during phagocytosis of live P. aeruginosa, facilitated in part by the ability of Rin1 to interact with Ras as an effector protein and regulate Rab5 nucleotide exchange activity (Pohlmann, Boeker et al. 1995).

With the growing demand for alternative therapeutic approach, many scientists are exploring alternative folk remedies to combat resilient pathogenic infections. Antimicrobial peptides (Eckert, Brady et al. 2006; Kapoor, Wadman et al. 2011) and herbal extracts (Adonizio, Kong et al. 2008; Song, Kong et al. 2010) are popular among many other approaches against $P$. aeruginosa infection. Here, in this part of the thesis, the antimicrobial and anti virulent activity of Dehydroleucodine (DhL) was tested against different strains of $P$. aeruginosa. DhL is a sesquiterpene lactone found in Artemisia douglasiana plant as secondary metabolites. Anti microbial activity of DhL has been established in Leishmania mexicana and Helicobactor pylori infections. DhL was found to be effective as an antimicrobial agent showing more susceptible toward PA103 and PA01 strains (MIC $0.48 \mathrm{mg} / \mathrm{ml}$ ), comparatively less virulent strain rather than PA14, (MIC, $0.96 \mathrm{mg} / \mathrm{ml}$ ) a more virulent strain. Also a prominent anti-virulent activity of $\mathrm{DhL}$ was established through various laboratory experiments against $P$. aeruginosa virulent 
factors such as LasA staphylolytic activity and LasB elastolytic activity, Pyocyanin secretion, interrupted attachment phase during biofilm formation and a significant reduction of T3S effector ExoS formation when P. aeruginosa strain PA01 was grown in presence of a threshold level of $\mathrm{DhL}\left(\mathrm{MIC}_{50}, 0.12 \mathrm{mg} / \mathrm{ml}\right)$. A sharp reduction in $P$. aeruginosa induced apoptosis was also observed when DhL treated PA01 were cocultured with J774-eclone macrophages. Although, a strong antimicrobial activity of DhL against $P$. aeruginosa was documented, electron microscopic studies showed no significant change or damage of bacterial cell due to the treatment. Hence, a detailed analysis of mechanism by which $\mathrm{DhL}$ affects $P$. aeruginosa bacterial cells awaits understanding of the proper mode of action.

\subsection{Future directions}

Although this dissertation provides a new insight on the modulation of Rab5 by $P$. aeruginosa during phagocytosis and a novel Rin $1 /$ Ras mediated pathway to facilitate the re-establishment of impaired Rab5 activity, this body of work remains from answering some interesting questions worthy for future investigation.

First, given that Rab5 is a target for ExoS ADP-ribosylation activity, it will be interesting to follow up the study to observe the interaction between ExoS and Rab5. Most importantly, to document if the phenomena is controlled by internalized bacteria or by extracellular bacteria targeting Rab5 on the surface of cell membrane with their Type III injecting apparatus.

Second, stretching the study from early phagocytosis to phago-lysosome formation, further investigation on degradation of internalized $P$. aeruginosa will be beneficial for therapeutic strategies. 
Third, a very crucial aspect of Rab5 modification remains unanswered in this study. The target arginines during Rab5 ADP-ribosylation will clearly demonstrate the mechanism of Rab5 inactivation/ Rab5-GEF interaction with Rab5/ Rab5-effector interaction with Rab5 post modification. This analysis not only holds importance for drug targeting, but also a crucial point of observation in respect to evolution of host-pathogen interactions.

Fourth, in the study of Ras/Rin1 facilitated Rab5 activation, in vivo analysis of ADPribosylated Ras and Rab5 interaction with Rin1 will be of high significance to understand the molecular mechanism of this novel pathway.

Finally, from the second part of this thesis, the molecular mechanism of antimicrobial activity showed by DhL on P. aeruginosa infection remains under speculation. Also, for the advancement of knowledge, the in vitro studies should be replicated in an animal model to establish DhL as a therapeutic agent in $P$. aeruginosa infection. 


\subsection{Figure legands}

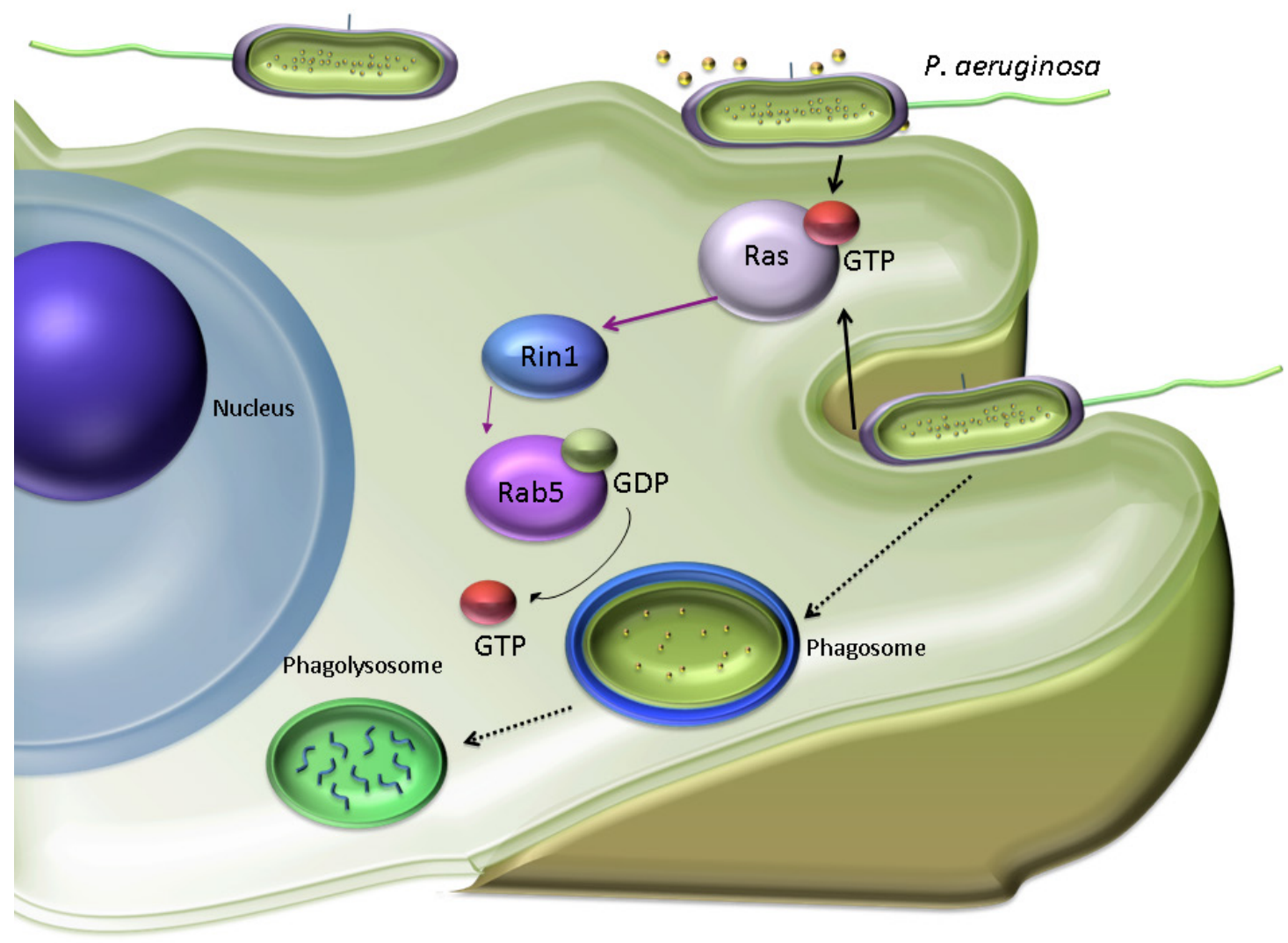

Figure 36: Model for Ras and Rin1 facilitated Rab5 activation during $P$. aeruginosa phagocytosis.

Role of activated Ras during phagocytosis as speculated by (Botelho, Harrison et al. 2009) indicates activation MEK1/ERK1 pathway aided by Ras effector Raf1 protein. During phagocytosis of live $P$. aeruginosa, Ras activation has also been indicated ( $\mathrm{Li}$, Feng et al. 1998). When Rin1 is over expressed, an alternative pathway has been speculated in macrphages (indicated by purple arrows), involving activated Ras and effector Rin1 interaction which in turn catalyzes Rab5 nucleotide exchange (GDP $\rightarrow$ GTP) creating an intrinsic Rab5 activation cycle. 


\subsection{References}

Adonizio, A., K. F. Kong and K. Mathee (2008). "Inhibition of quorum sensingcontrolled virulence factor production in Pseudomonas aeruginosa by South Florida plant extracts." Antimicrob Agents Chemother 52(1): 198-203.

Apodaca, G., M. Bomsel, R. Lindstedt, J. Engel, D. Frank, K. E. Mostov and J. WienerKronish (1995). "Characterization of Pseudomonas aeruginosa-induced MDCK cell injury: glycosylation-defective host cells are resistant to bacterial killing." Infect Immun 63(4): 1541-1551.

Barbieri, A. M., Q. Sha, P. Bette-Bobillo, P. D. Stahl and M. Vidal (2001). "ADPribosylation of Rab5 by ExoS of Pseudomonas aeruginosa affects endocytosis." Infect Immun 69(9): 5329-5334.

Bendig, J. W., P. W. Kyle, P. L. Giangrande, D. M. Samson and B. S. Azadian (1987). "Two neutropenic patients with multiple resistant Pseudomonas aeruginosa septicaemia treated with ciprofloxacin." J R Soc Med 80(5): 316-317.

Carmeli, Y., N. Troillet, G. M. Eliopoulos and M. H. Samore (1999). "Emergence of antibiotic-resistant Pseudomonas aeruginosa: comparison of risks associated with different antipseudomonal agents." Antimicrob Agents Chemother 43(6): 1379-1382.

Celli, J. and B. B. Finlay (2002). "Bacterial avoidance of phagocytosis." Trends Microbiol 10(5): 232-237.

Chitkara, Y. K. and T. C. Feierabend (1981). "Endogenous and exogenous infection with Pseudomonas aeruginosa in a burns unit." Int Surg 66(3): 237-240.

Cowell, B. A., D. Y. Chen, D. W. Frank, A. J. Vallis and S. M. Fleiszig (2000). "ExoT of cytotoxic Pseudomonas aeruginosa prevents uptake by corneal epithelial cells." Infect Immun 68(1): 403-406.

Deng, Q. and J. T. Barbieri (2008). "Molecular mechanisms of the cytotoxicity of ADPribosylating toxins." Annu Rev Microbiol 62: 271-288.

Duclos, S., R. Diez, J. Garin, B. Papadopoulou, A. Descoteaux, H. Stenmark and M. Desjardins (2000). "Rab5 regulates the kiss and run fusion between phagosomes and endosomes and the acquisition of phagosome leishmanicidal properties in RAW 264.7 macrophages." J Cell Sci 113 Pt 19: 3531-3541.

Eckert, R., K. M. Brady, E. P. Greenberg, F. Qi, D. K. Yarbrough, J. He, I. McHardy, M. H. Anderson and W. Shi (2006). "Enhancement of antimicrobial activity against Pseudomonas aeruginosa by coadministration of G10KHc and tobramycin." Antimicrob Agents Chemother 50(11): 3833-3838. 
Franzetti, F., M. Cernuschi, R. Esposito and M. Moroni (1992). "Pseudomonas infections in patients with AIDS and AIDS-related complex." J Intern Med 231(4): 437-443.

Fraylick, J. E., E. A. Rucks, D. M. Greene, T. S. Vincent and J. C. Olson (2002). "Eukaryotic cell determination of ExoS ADP-ribosyltransferase substrate specificity." Biochem Biophys Res Commun 291(1): 91-100.

Frithz-Lindsten, E., Y. Du, R. Rosqvist and A. Forsberg (1997). "Intracellular targeting of exoenzyme $\mathrm{S}$ of Pseudomonas aeruginosa via type III-dependent translocation induces phagocytosis resistance, cytotoxicity and disruption of actin microfilaments." Mol Microbiol 25(6): 1125-1139.

Hueck, C. J. (1998). "Type III protein secretion systems in bacterial pathogens of animals and plants." Microbiol Mol Biol Rev 62(2): 379-433.

Kapoor, R., M. W. Wadman, M. T. Dohm, A. M. Czyzewski, A. M. Spormann and A. E. Barron (2011). "Antimicrobial peptoids are effective against Pseudomonas aeruginosa biofilms." Antimicrob Agents Chemother 55(6): 3054-3057.

Keyser, P., M. Elofsson, S. Rosell and H. Wolf-Watz (2008). "Virulence blockers as alternatives to antibiotics: type III secretion inhibitors against Gram-negative bacteria." J Intern Med 264(1): 17-29.

Laing, S., M. Unger, F. Koch-Nolte and F. Haag (2011). "ADP-ribosylation of arginine." Amino Acids 41(2): 257-269.

Lister, P. D., D. J. Wolter and N. D. Hanson (2009). "Antibacterial-resistant Pseudomonas aeruginosa: clinical impact and complex regulation of chromosomally encoded resistance mechanisms." Clin Microbiol Rev 22(4): 582-610.

Lyczak, J. B., C. L. Cannon and G. B. Pier (2000). "Establishment of Pseudomonas aeruginosa infection: lessons from a versatile opportunist." Microbes Infect 2(9): 10511060.

Nicas, T. I., D. W. Frank, P. Stenzel, J. D. Lile and B. H. Iglewski (1985). "Role of exoenzyme $\mathrm{S}$ in chronic Pseudomonas aeruginosa lung infections." Eur J Clin Microbiol 4(2): 175-179.

Pallen, M. J., S. A. Beatson and C. M. Bailey (2005). "Bioinformatics, genomics and evolution of non-flagellar type-III secretion systems: a Darwinian perspective." FEMS Microbiol Rev 29(2): 201-229.

Pohlmann, R., M. W. Boeker and K. von Figura (1995). "The two mannose 6-phosphate receptors transport distinct complements of lysosomal proteins." J Biol Chem 270(45): 27311-27318. 
Roberts, R. L., M. A. Barbieri, J. Ullrich and P. D. Stahl (2000). "Dynamics of rab5 activation in endocytosis and phagocytosis." J Leukoc Biol 68(5): 627-632.

Song, Z., K. F. Kong, H. Wu, N. Maricic, B. Ramalingam, H. Priestap, L. Schneper, J. M. Quirke, N. Hoiby and K. Mathee (2010). "Panax ginseng has anti-infective activity against opportunistic pathogen Pseudomonas aeruginosa by inhibiting quorum sensing, a bacterial communication process critical for establishing infection." Phytomedicine 17(13): 1040-1046.

Wang, Y., R. T. Waldron, A. Dhaka, A. Patel, M. M. Riley, E. Rozengurt and J. Colicelli (2002). "The RAS effector RIN1 directly competes with RAF and is regulated by 14-3-3 proteins." Mol Cell Biol 22(3): 916-926. 
VITA

\section{SUSHMITA MUSTAFI}

Born, Calcutta, India

2000-2003

B.S., Zoology

Calcutta University, West Bengal, India

2003-2005

M.S., Zoology

Calcutta University, West Bengal, India

2004-2005

Research Assistant, Dept of Microbiology, Bose Institute. Calcutta, West Bengal, India

2005-2006

Research Assistant, Indian Institute of Chemical Biology Calcutta, West Bengal, India.

2006-2007

Research assistant, Texas Tech, Lubbock, Texas

2008-2013

Doctoral Candidate

Florida International University

Miami, Florida

$2008-2013$

Teaching Assistant, Dept. of Biology Florida International University Miami, Florida.

\section{PUBLICATIONS AND PRESENTATIONS}

Mustafi S, Kim LW, Barbieri MA. "Microorganisms: Modulation of phagocytosis and its Mechanism of Action". Curr Cell Biochem 2013; 3(1):1-10.

Mustafi S, Rivero N, Olson J, Stahl P, Barbieri MA. "Regulation of Rab5 function during phagocytosis of live Pseudomonas aeruginosa in macrophages." Infection and immunity $2013 \mathrm{Jul} ; 81(7): 2426-36$ 
Modulation of early phagocytic pathway in mouse macrophage during Pseudomonas aeruginosa invasion. S. Mustafi. M.A. Barbieri, Gordon Conference, Phagocytes, 2013. New Hampshire.

Role of Ras interference 1 (Rin1) in Pseudomonas aeruginosa phagocytosis. Mustafi S, Barbieri MA. ASM 2013, Denver, Co.

Inactivation of Rab5 during interaction of live Pseudomonas aeruginosa with macrophage, FIU Biology symposium 2013.

Macrophage- Rab5 interaction during phagocytosis of live Pseudomonas aeruginosa. Mustafi S, Barbieri MA. ASM 2012, San Francisco, Ca.

Inhibition of Rab5 activation by Exotoxin S of Pseudomonas aeruginosa. S. Mustafi, N. Rivero, M.A. Barbieri, ASCB 2011, Denver, Co.

Modulation of the small GTPase Rab5 activity during Pseudomonas aeruginosa invasion. S.Mustafi, M.A. Barbieri, ASCB 2010, Phyladelphia, Pa.

Identification of modulation of early endosomal GTPase Rab5 by Pseudomonas aeruginosa. Oral presentation at biomedical comparative immunology symposium, FIU, 2009.

Poster presentation on National symposium on ZOOLOGY-A NEORIALISTIC APPROACH organized by Dept. of Zoology, University of Calcutta 2004.

Participant in NATIONAL SEMINAR ON EVOLUTIONARY BIOLOGY AND BIOTECHNOLOGY organized by ZOOLOGICAL SOCIETY, Calcutta and Zoological survey of India 2004. 